\title{
Digital Model Predictive Control for Multi-Port DC-DC Converters
}

\author{
Wang Benfei
}

School of Electrical and Electronic Engineering

A thesis submitted to the Nanyang Technological University in fulfillment of the requirement for the degree of

Doctor of Philosophy 


\section{ACKNOWLEDGEMENTS}

First and foremost, I would like to express my deepest thanks to my supervisor Associate Professor So Ping Lam. When I made a decision to transfer from Division of Circuits and Systems to Division of Power Engineering, he generously accepted me as his $\mathrm{PhD}$ student and helped me begin a new research topic. Throughout my $\mathrm{PhD}$ studies, he provides the valuable and excellent guidance and suggestion to help me overcome the problems I encounter, and I benefit a lot from the kind and patient discussions with him. When I meet up with challenges in my research, he always encouraged me to regain the confidence. Moreover, his rigorous academic attitude sets me an example of a scientific researcher and reminds me to pursue higher goals.

Then I would like to thank Tan Kuan Tak, Peng Xiaoyang, Ravi Kishore, Xian Liang and Nandha Kumar, who are my fellows. Tan Kuan Tak offers me his help during my whole $\mathrm{PhD}$ studies. He generously shares his experience on research and makes the example of a good $\mathrm{PhD}$ student for me. Moreover, he patiently participates in all my papers including writing and revision. Peng Xiaoyang provides me assistance in learning and applying the model predictive control theory, which is the fundamental theory for most of my research works. Ravi Kishore works with me on the DSP development environment setup and programming for the hardware implementation. Xian Liang collaborates with me in my last two projects involving circuit design and experimental operation, and he also provides his valuable advice on the paper writing. Nanda Kumar generally shares the smart grid data to help me study the influence of my work on the grid, which makes my research more thorough. I would also like to thank my group members for the in-depth and fruitful discussions on the project. They are Wang Yu, Wang Zhe, Thomas John, and Dr. Sivaneasan Balakrishnan. Without their help, I cannot work on my research smoothly.

Furthermore, I would like to take this opportunity to express my special thanks to the laboratory staffs Chia-Nge Tak Heng and Foo Mong Keow, Thomas in Clean Energy Research Laboratory, and Yim-Koh Swee Eng in Energy and Machines Laboratory. They generously offer their generous help in software and hardware for my research works. It is their careful administration that makes the laboratory a comfortable and convenient place for research. 
I would like to thank Nanyang Technological University (NTU). NTU offers me the research scholarship, which enables the chance for me to pursue the doctoral degree.

Last but not least, I need to thank my family. It is the understanding of my family that supports me to pursue the doctoral degree on abroad alone. No matter what problems I confront during my research or life, I can re-gain the courage to solve them after their enlightenment and encouragement. 


\begin{abstract}
This thesis presents several digital model predictive control (MPC) methods for different types of multi-port DC-DC converters, including single-inductor single-input multiple-output (SI-SIMO) DC-DC converter, single-inductor multiple-input multipleoutput (SI-MIMO) DC-DC converter and single-input dual-output (SIDO) flyback converter.

The multi-port DC-DC converters researched in this thesis have a common characteristic, i.e., the adoption of single inductor/transformer is implemented in the converters. Therefore, all loads driven by these multi-port DC-DC converters share the same inductor/transformer. Compared to the conventional multiple-load system using several single-input single-output (SISO) converters, this single inductor/transformer topology contributes to the reduction of component number, circuit size and cost. Moreover, the single inductor/transformer will also relieve the electromagnetic interference (EMI) problem, which is serious resulting from massive inductors/transformers in the multiple-load system with lots of SISO converters.

The only disadvantage of single inductor/transformer is that the mutual interference among the loads, i.e., the cross regulation. Since there is residual energy stored in the shared inductor/transformer after the completion of driving one load, the residual energy will be delivered when driving next load, which means that it is influenced by the previous load. Consequently, the loads are supplied with mismatching energy rather than their corresponding demands. To solve the cross regulation problem, control methods with fast and dynamic response ability are imperative, which can provide optimal control actions to supply loads with the corresponding power requirements based on real-time data. Therefore, the cross regulation can be significantly reduced. MPC is an effective and robust method with the aforementioned fast and dynamic response capability. This method requires a state-space model of the targeted system. Then it is able to generate the optimal control actions in advance based on the statespace model, cost function, references and real-time states of the target system. The optimal control actions will be applied to the target system and the whole process will be repeated in the manner of receding horizon. Therefore, this MPC strategy has the capability to regulate the target system to track the references closely.
\end{abstract}


For the SI-SIMO DC-DC converter, model predictive voltage control (MPVC) method and model predictive current control (MPCC) method are developed to suppress the cross regulation.

In this thesis, the design of the proposed MPVC method with augmented state-space model, cost function and constraints for the SI-SIMO DC-DC converters are discussed. The simulation and hardware platforms of single-inductor single-input dual-output (SISIDO) buck converter are developed and the proposed the MPVC method is implemented. Simulation of the influences of MPVC method parameters, such as predict horizon, control horizon and Lagrange multiplier, is conducted to guide the control parameters setting for hardware implementation. Steady-state operation and dynamic performance of the proposed MPVC method are also simulated. Moreover, several experimental cases are conducted to study the performance of the proposed method based on the simulation results and the SI-SIDO buck converter hardware prototype. Simulation and experimental results demonstrate that the proposed MPVC method guarantees low cross regulation for the SI-SIMO DC-DC converter and has the capability to respond to variations in load and reference rapidly.

The development of the MPCC method is also presented in this thesis. Furthermore, a charge equalization method for the series-connected batteries in electric vehicles (EVs) is proposed based on the MPCC method. The imbalance of batteries in EVs resulting from manufacturing differences and cycles of charging and discharging will shorten the lifetime of battery cells. Therefore, charging equalization is the most important factor for the lifetime extension of batteries. A possible charging equalization method is an individual charging operation based on the state-of-charge (SOC) of each battery cell. In order to realize the individual charging, SI-SIMO DC-DC converter using MPCC method is employed. Similar to the MPVC method, the MPCC method can regulate the output currents to drive loads and reduce the cross regulation. Since the battery charging is split into the constant current (CC) charging and the constant voltage (CV) charging, the corresponding current reference assignment algorithms in the $\mathrm{CC}$ stage and the $\mathrm{CV}$ stage are also developed to provide the proper reference for the MPCC method. Simulation studies in different SOC situations are conducted to verify the performance of the proposed charge equalization method, and the results indicate that it is able to balance the series-connected batteries successfully. 
The MPC method is extended to control the SI-MIMO DC-DC converter in this thesis. This SI-MIMO DC-DC converter also has the cross regulation problem resulting from the adoption of single inductor. Different from the SIMO topology, the MIMO topology also needs the regulation of input power considering the multiple power sources. Hence, a power sharing and cross regulation suppression method is developed for the SIMIMO DC-DC converter. The proposed method is based on the MPC method, power sharing method and time-multiplexing method. The power sharing controller is developed to regulate the power from the multiple power sources, and the cross regulation suppression controller is designed to control the output voltages independently with reduced cross regulation. In order to perform the validation of the proposed method, simulation and experimental platforms are built. Several simulation and experimental cases, including steady-state operation and dynamic performance, are conducted, and the results demonstrate that the proposed method is able to regulate the SI-MIMO DC-DC converter effectively and robustly.

Finally, a dynamic model predictive voltage control (DMPVC) technique for the SIDO flyback converter is presented. Cross regulation is still the critical problem because two secondary coils share the same primary coil in the transformer of the SIDO flyback converter. The proposed the DMPVC technique has the ability to reduce cross regulation by generating the optimal control signals dynamically. The state-space models of flyback for DMPVC method are formulated, as well as the corresponding cost function which defines the optimal control actions. The verification of the proposed DMPVC technique is conducted based on a simulation platform built in Simulink. With the proposed DMPVC method, the SIDO flyback converter is able to work in buckbuck, buck-boost and boost-boost modes, and complete the mode transformation successfully. 


\section{TABLE OF CONTENTS}

ACKNOWLEDGEMENTS

i

ABSTRACT

TABLE OF CONTENTS

LIST OF FIGURES

LIST OF TABLES

LIST OF ABBREVIATIONS

CHAPTER 1 INTRODUCTION

1.1 BACKGROUND AND MOTIVATION

1.1.1 INTRODUCTION OF HYBRID RENEWABLE ENERGY SYSTEMS

1.1.2 INTRODUCTION OF EVS

1.1.3 Motivations

1.2 OBJECTIVES OF THE THESIS

1.3 Contributions of The Thesis

1.4 Organization of The Thesis

CHAPTER 2 LITERATURE REVIEW

2.1 Multi-PORT DC-DC CONVERTER

2.1.1 MISO DC-DC CONVERTER

2.1.2 SIMO DC-DC CONVERTER

2.1.3 SIMO FLYBACK CONVERTER

2.1.4 MIMO DC-DC CONVERTER

2.2 Charging Equalization

2.3 MOdEL PREDICTIVE CONTROL

iii

vi

$\mathbf{x}$

xiv

Xv

1

1

1

4

7

7

9

10

13

13

13

16

20

22

24

27

CHAPTER 3 MODEL PREDICTIVE VOLTAGE CONTROL FOR SI-SIMO DC-DC CONVERTER

3.1 STATE-SPACE MODELS AND CONSTRAINTS ANALYSIS 
3.1.1 SI-SIMO DC-DC BUCK CONVERTER 33

3.1.2 STATE-SPACE MODEL FOR MPVC METHOD 33

3.1.3 CONSTRAINTS FOR MPVC METHOD 36

$\begin{array}{ll}3.2 & \text { MPVC METHOD } \\ \end{array}$

$\begin{array}{lll}3.2 .1 & \text { MPVC CONTROLLER } & 37\end{array}$

3.2.2 ARCHITECTURE OF SI-SIMO DC-DC CONVERTER WITH MPVC

$\begin{array}{ll}\text { CONTROLLER } & 38\end{array}$

3.2.3 CONTROL SCHEME OF MPVC METHOD 39

3.2.4 DESIGN CONSIDERATION FOR MPVC METHOD 40

$\begin{array}{lll}3.3 & \text { Simulation RESUltS } & 41\end{array}$

3.3.1 Steady-STATE OF MPVC Method 41

3.3.2 Dynamic Performance of MPVC Method 43

3.4 EXPERIMENTAL RESULTS 46

3.4.1 STEADY-STATE OF MPVC METHOD $\quad 47$

3.4.2 DYNAMIC PERFoRmanCE OF MPVC MeThOD 49

$\begin{array}{lll}3.5 & \text { CONCLUSION } & 54\end{array}$

CHAPTER 4 MODEL PREDICTIVE CURRENT CONTROL FOR CHARGING EQUALIZATION BASED ON SI-SIMO DC-DC CONVERTER

4.1 MPCC METHOD 55

4.1.1 ARChITECTURE OF SI-SIMO DC-DC BUCK CONVERTER FOR CHARGING EQUALIZATION

4.1.2 MPCC METHOD $\quad 55$

4.2 Charging Equalization Method $\quad 59$

4.2.1 Algorithm FOR CC ChARgING

$\begin{array}{lll}\text { 4.2.2 AlgorithM FOR CV CHARgING } & 61\end{array}$

$\begin{array}{lll}\text { 4.2.3 Charge Equalization Míthod } & 63\end{array}$

$\begin{array}{lll}4.3 & \text { Simulation Studies } & 64\end{array}$

vii 
4.3.1 CC ChARgING ANd CV Charging BASEd ON MPCC METHOD 64

4.3.2 MPCC FOR SI-SIMO DC-DC BUCK CONVERTER 65

4.3.3 Charge Equalization Performance Based on SI-Simo DC-DC BUCK CONVERTER

4.3.4 COMPARISON WITH EXISTING METHOdS For Charging Equalization 72

4.4 ConClusion 72

CHAPTER 5 POWER SHARING AND CROSS REGULATION SUPPRESSION METHOD FOR SI-MIMO DC-DC CONVERTER 74

$\begin{array}{lll}5.1 & \text { OpERATIONAL PRINCIPLES } & 74\end{array}$

$\begin{array}{lll}\text { 5.1.1 SI-MIMO DC-DC BUCK CONVERTER } & 74\end{array}$

5.1.2 MPC FOR SI-MIMO BUCK CONVERTER

$\begin{array}{lll}\text { 5.1.3 POWER SHARING METHOD } & 78\end{array}$

$\begin{array}{lll}\text { 5.1.4 Time-MultiPleXing METHOD } & 80\end{array}$

$\begin{array}{ll}5.2 \text { CONTROLler ANd COMPONENT Design } & 81\end{array}$

$\begin{array}{lll}\text { 5.2.1 Power SHaring CONTROLler } & 81\end{array}$

5.2.2 Cross Regulation SuPPRESSION CONTROLLER 82

$\begin{array}{lll}\text { 5.2.3 COMPONENT Design CONSIDERATION } & 83\end{array}$

5.3 Simulation Results $\quad 85$

$\begin{array}{llr}\text { 5.3.1 STEADY-STATE OPERATION } & 85\end{array}$

$\begin{array}{ll}\text { 5.3.2 Dynamic PERFormance } & 87\end{array}$

5.4 EXPERIMENTAL RESULTS

5.4.1 StEADY-STATE OPERATION 92

$\begin{array}{lll}\text { 5.4.2 Dynamic Performance } & 95\end{array}$

$\begin{array}{ll}5.5 \text { CONCLUSION } & 99\end{array}$

CHAPTER 6 DYNAMIC MODEL PREDICTIVE VOLTAGE CONTROL FOR $\begin{array}{lr}\text { SIDO FLYBACK CONVERTER } & 101\end{array}$

6.1 State-SPaCe Models And Constraints Analysis 101 
6.1.1 SIDO FLYBACK CONVERTER 101

6.1.2 StATE-SPACE MODEl FOR Flyback CONVERTER 101

6.1.3 Priority CalCUlation Algorithm AND CONSTRAints 104

6.2 DMPVC METHOD FOR SIDO FLYBACK CONVERTER 106

$\begin{array}{lll}\text { 6.3 Simulation Results } & 106\end{array}$

$\begin{array}{lll}\text { 6.3.1 BUCK-BUCK MODE } & 107\end{array}$

$\begin{array}{lll}\text { 6.3.2 } & \text { BUCK-BOOST MODE } & 108\end{array}$

$\begin{array}{lll}\text { 6.3.3 } & \text { Boost-Boost Mode } & 110\end{array}$

$\begin{array}{lll}\text { 6.3.4 Mode Transition } & 111\end{array}$

$\begin{array}{ll}6.4 \text { CONCLUSION } & 113\end{array}$

CHAPTER 7 CONCLUSIONS AND FUTURE WORKS 114

$\begin{array}{ll}7.1 \text { Conclusions } & 114\end{array}$

$\begin{array}{lll}7.2 & \text { FUTURE WORKS } & 117\end{array}$

$\begin{array}{lll}\text { 7.2.1 Charging Equalization SyStem } & 117\end{array}$

7.2.2 DMPVC METHOD FOR SIDO FLYBACK CONVERTER 118

7.2.3 POWER SHARING AND DMPVC METHOD FOR MIMO FLYBACK

$\begin{array}{ll}\text { CONVERTER } & 118\end{array}$

7.2.4 APPLICATION OF MULTI-PORT DC-DC CONVERTER IN PRACTICAL HRESS

7.2.5 Parameter Selection Using Particle SWarm Optimization 119

$\begin{array}{lr}\text { REFERENCES } & 120\end{array}$ 


\section{LIST OF FIGURES}

Figure 1-1: Percentage of world renewable electricity generation over the total electricity generation [5]

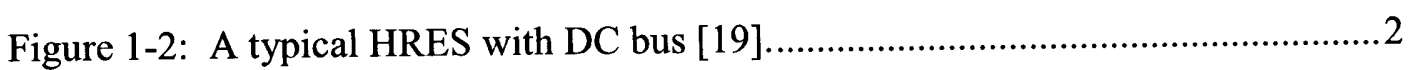

Figure 1-3: A standalone wind-solar system with dump power control [29]............... 3

Figure 1-4: A typical HRES using multi-port DC-DC converter. .............................. 4

Figure 1-5: EV charging station deploying standalone PV panel on rooftop [41] .......5

Figure 1-6: EV productions, up row: Nissan Leaf, Honda Fit EV and Ford Focus

Electric; down row: BYD E6 and Tesla Model S....................................................6

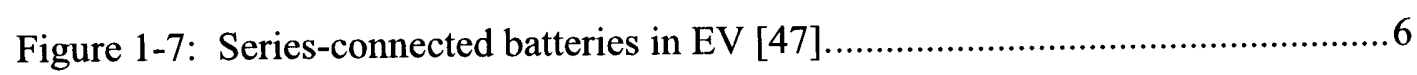

Figure 2-1: The architecture of a typical MISO boost converter [55] ........................ 14

Figure 2-2: The proposed SI-SDIO boost converter [65]. (a) Architecture of the SI-

SIDO boost converter. (b) Timing diagram of the SI-SIDO boost converter...............16

Figure 2-3: The proposed SI-SDIO boost converter with a freewheeling switch [66].

(a) Architecture of the SI-SIDO boost converter with a freewheeling switch. (b) Timing

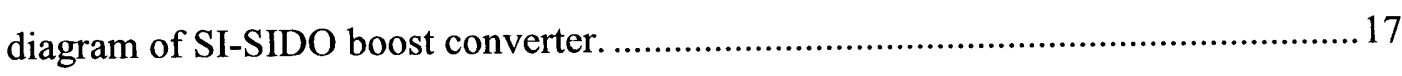

Figure 2-4: The architecture of the SI-MIMO DC-DC converter [95], [96].............23

Figure 2-5: Automatic charge equalization circuit based on regulated voltage source

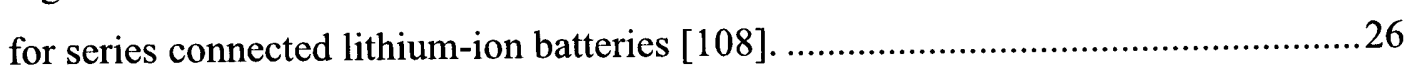

Figure 2-6: A general control diagram of FS-MPC for power converters [112], [113]. .28

Figure 3-1: Architecture of SI-SIMO DC-DC buck converter. ................................... 34

Figure 3-2: Block diagram of MPVC controller....................................................... 38

Figure 3-3: Architecture of SI-SIMO DC-DC buck converter with MPVC controller.

Figure 3-4: MPVC control scheme for single-inductor single-input dual-output (SISIDO) buck converter.

Figure 3-5: Influences of the control horizon $N_{C}$ and Lagrange multiplier $\lambda$ of MPVC method when $T_{S}=5 \mu \mathrm{s}$ and $V_{R e f}=5 \mathrm{~V}$. (a) Influence of $N_{C}$ and $\lambda$ when $N_{P}=12$. (b) Influence of $N_{P}$ when $N_{C}=1, \lambda=0.001$.

Figure 3-6: Steady-state simulation waveforms of the proposed MPVC method based on SI-SIDO buck converter as shown in Figure 3-1 
Figure 3-7: Transient response to the reference change simulation waveforms of the proposed MPVC method based on SI-SIDO buck converter as shown in Figure 3-1.44 Figure 3-8: Load regulation simulation waveforms of the proposed MPVC method based on the SI-SIDO buck converter as shown in Figure 3-1. (a) Step change in $R_{1}$. (b) Step change in $R_{2}$. .45

Figure 3-9: Hardware platform for verification of the proposed MPVC method.......46 Figure 3-10: Steady-state waveforms of the proposed MPVC method. Output voltages $v_{1}, v_{2} \sim 2 \mathrm{~V} / \mathrm{div}$, Output currents $i_{1}, i_{2} \sim 200 \mathrm{~mA} / \mathrm{div}$, time $\sim 10 \mu \mathrm{s} / \mathrm{div}$

Figure 3-11: Steady-state waveforms of selection signals $S_{1}$ and $S_{2}$, control signal $S$ and inductor current $i_{L}$. .48

Figure 3-12: Transient response to reference voltage variation waveforms of the proposed MPVC method based on SI-SIDO buck converter. Output voltages $v_{1}$, $v_{2} \sim 2 \mathrm{~V} / \mathrm{div}$, output currents $i_{1}, i_{2} \sim 200 \mathrm{~mA} / \mathrm{div}$, time $\sim 500 \mu \mathrm{s} / \mathrm{div}$. .50

Figure 3-13: Load regulation waveforms of the proposed MPVC method. $V_{1}$, $V_{2} \sim 2 \mathrm{~V} / \mathrm{div}, I_{1}, I_{2} \sim 1 \mathrm{~A} / \mathrm{div}$, time $\sim 10 \mathrm{~ms} /$ div. (a) Step change in $R_{1}$. (b) Step change in $R_{2}$.

Figure 3-14: Efficiency versus total load current of the proposed MPVC method based on the SIDO buck converter. .52

Figure 4-1: Battery charging equalization system architecture based on the SI-SIMO DC-DC buck converter. .56

Figure 4-2: Block diagram of the MPCC controller structure. .58

Figure 4-3: CC charging flowchart. A and B are the symbols of the related arrows. Initial current reference assignment algorithm for $\mathrm{CC}$ and current reference reassignment algorithm for $\mathrm{CC}$ are continued in Figure 4-4. .59

Figure 4-4: (a) Initial current reference assignment algorithm for CC charging. (b) Current reference re-assignment algorithm for $\mathrm{CC}$ charging. $\mathrm{C}$ and $\mathrm{D}$ are the symbols of the related arrows.

Figure 4-5: (a) CV charging flowchart. (b) Current reference assignment algorithm for $\mathrm{CV}$ charging. $\mathrm{E}$ and $\mathrm{F}$ are the symbols of the related arrows.

Figure 4-6: SI-SIMO DC-DC buck converter controller for charge equalization......63

Figure 4-7: CC charging and CV charging waveforms based on the MPCC method.64 Figure 4-8: Comparison of the output currents without and with the MPCC method based on SI-SIDO buck converter. (a) A typical cross regulation phenomenon without the MPCC method. (b) Simulation results based on the MPCC method. .65 
Figure 4-9: Control signals for the SI-SIMO DC-DC buck converter with MPCC. ..66

Figure 4-10: SOC curves for small SOC difference condition. ...................................68

Figure 4-11: Current curves for small SOC difference condition...............................68

Figure 4-12: Voltage curves for small SOC difference condition. .............................68

Figure 4-13: SOC curves for large SOC difference condition.................................. 70

Figure 4-14: Current curves for large SOC difference condition. ..............................70

Figure 4-15: Voltage curves for large SOC difference condition.............................70

Figure 5-1: Architecture of the SI-MIMO buck converter. …..................................75

Figure 5-2: Operation of the SI-MIMO buck converter. (a) $S_{i n, i}$ and $S_{\text {out }, j}$ are on, the other switches are off. (b) $S_{\text {out } j}$ is on, the other switches are off..................................75

Figure 5-3: Block diagram of MPC method for SISO buck converter. ......................77

Figure 5-4: Illustration of power sharing control. (a) Block diagram of PI-based method.

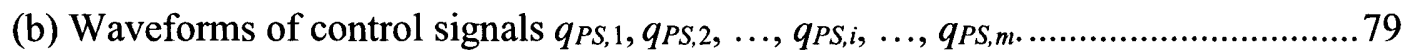

Figure 5-5: Overview of the proposed power sharing and cross regulation suppression method. . .81

Figure 5-6: Power sharing control signal waveforms based on SI-DIDO buck converter.

Figure 5-7: Cross regulation suppression control signal waveforms based on SI-DIDO buck converter.

Figure 5-8: Simulation waveforms of $\bar{i}_{i_{n, 1},}, \bar{i}_{i n, 2}, v_{o u t, 1}$ and $v_{o u t, 2}$ in steady-state operation of the proposed method in $v_{i n, 1}=24 \mathrm{~V}, v_{i n, 2}=20 \mathrm{~V}$ condition. (a) $v_{\text {out }, 1}=12.0 \mathrm{~V}$ and $v_{\text {out }, 2}=8.0 \mathrm{~V}$. (b) $v_{\text {out }, 1}=12.0 \mathrm{~V}$ and $v_{\text {out }, 2}=10.0 \mathrm{~V}$.

Figure 5-9: Simulation waveforms of $\bar{i}_{i, 1}, \bar{i}_{i, 2}, v_{o u t, 1}$ and $v_{o u t, 2}$ in steady-state operation of the proposed method under $v_{i n, 1}=30 \mathrm{~V}, v_{i n, 2}=20 \mathrm{~V}$ condition. (a) $v_{o u t, 1}=12.0 \mathrm{~V}$ and $v_{\text {out }, 2}=8.0 \mathrm{~V}$. (b) $v_{\text {out }, 1}=12.0 \mathrm{~V}$ and $v_{\text {out }, 2}=10.0 \mathrm{~V}$. .86

Figure 5-10: Line regulation simulation results of the proposed method. .87

Figure 5-11: Load regulation simulation results of the proposed method. . .88

Figure 5-12: Simulation results of response to output voltage reference step change. .88

Figure 5-13: Simulation results of response to input current reference step change. .88 Figure 5-14: Hardware platform for verification of the proposed method. . .90

Figure 5-15: Experimental waveforms of $\bar{i}_{m, 1}, \bar{i}_{i, 2}, v_{o u t, 1}$ and $v_{o u t, 2}$ in steady-state operation of the proposed method under $v_{i n, 1}=24 \mathrm{~V}, v_{i n, 2}=20 \mathrm{~V}$ conditions based on the 
SI-DIDO buck converter. (a) $v_{\text {out }, 1}=12.0 \mathrm{~V}$ and $v_{\text {out }, 2}=7.99 \mathrm{~V}$. (b) $v_{\text {out }, 1}=11.9 \mathrm{~V}$ and $v_{\text {out }, 2}=9.96 \mathrm{~V}$.

Figure 5-16: Experimental waveforms of $\bar{i}_{i, 1}, \bar{i}_{i, 2}, v_{o u t, 1}$ and $v_{\text {out }, 2}$ in steady-state operation of the proposed method under $v_{i n, 1}=30 \mathrm{~V}, v_{i n, 2}=20 \mathrm{~V}$ condition. (a) $v_{o u t, 1}=11.9 \mathrm{~V}$ and $v_{\text {out }, 2}=7.99 \mathrm{~V}$. (b) $v_{o u t, 1}=12.0 \mathrm{~V}$ and $v_{o u t, 2}=10.1 \mathrm{~V}$.

Figure 5-17: Waveforms of control signals $q_{P S, 1}, q_{P S, 2}, q_{1}^{\prime}, q_{1}$ and $q_{2}$ and inductor current $i_{l}$ under $v_{i n, 1}=24 \mathrm{~V}, v_{i n, 2}=20 \mathrm{~V}, v_{o u t, 1}=12.0 \mathrm{~V}$ and $v_{o u t, 2}=8.0 \mathrm{~V}$ condition. .94

Figure 5-18: Experimental results of line regulation of the proposed method based on SI-DIDO buck converter. .96

Figure 5-19: Experimental results of load regulation of the proposed method based on the SI-DIDO buck converter. .97

Figure 5-20: Experimental results of response to output voltage reference step change. .98

Figure 5-21: Experimental results of response to input current reference step change. .99

Figure 6-1: Architecture and operational states of the proposed SIDO flyback converter. (a) Architecture of SIDO flyback converter. (b) State 1. (c) State 2. (d) State 3....... 102 Figure 6-2: Current and control signal waveforms of the proposed flyback converter. 103

Figure 6-3: The proposed DMVPC controller for the SIDO flyback converter....... 105

Figure 6-4: Steady-state operation simulation results of the buck-buck mode......... 107

Figure 6-5: Load regulation simulation results of the buck-buck mode....................108

Figure 6-6: Steady-state operation simulation results of the buck-boost mode........ 109

Figure 6-7: Load regulation simulation results of the buck-boost mode................... 109

Figure 6-8: Steady-state operation simulation results of the boost-boost mode.......110

Figure 6-9: Load regulation simulation results of the boost-boost mode. 111

Figure 6-10: Mode transformation from buck-buck mode to buck-boost mode. .....112

Figure 6-11: Mode transformation from buck-boost mode to boost-boost mode.... 112 


\section{LIST OF TABLES}

Table 1-1 Range in Miles, Battery Types and Charging Types of EVs [44], [45] .......5

Table 3-1 Simulation parameters of the SIDO DC-DC buck converter ......................40

Table 3-2 Main components adopted in hardware platform .....................................46

Table 3-3 Comparison with Existing Methods for SIMO DC-DC Converter ............53

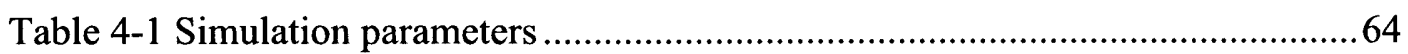

Table 4-2 Comparison of different charging equalization methods for " $n$ " series-

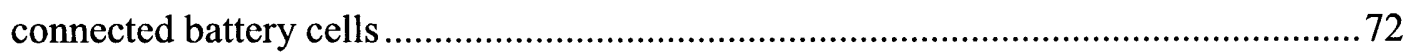

Table 5-1 Simulation parameters of the SI-DIDO buck converter ...............................84

Table 5-2 Main components adopted in hardware platform ......................................90

Table 6-1 Simulation parameters setting for DMPVC …........................................... 105 


\section{LIST OF ABBREVIATIONS}

\begin{tabular}{|c|c|c|}
\hline $\mathrm{ACC}$ & - & Adaptive Current Compensation \\
\hline AFE & - & Active Front-End \\
\hline BPM & - & Battery Power Module \\
\hline $\mathrm{CC}$ & - & Constant Current \\
\hline $\mathrm{CCM}$ & - & Continuous-Conduction Mode \\
\hline CDR & - & Common Duty Ratio \\
\hline CEA & - & Charge Equalization Algorithm \\
\hline $\mathrm{CV}$ & - & Constant Voltage \\
\hline DCM & - & Discontinuous-Conduction Mode \\
\hline DMPVC & - & Dynamic Model Predictive Voltage Control \\
\hline EMI & - & Electromagnetic Interference \\
\hline ESR & - & Equivalent Series Resistance \\
\hline EV & - & Electric Vehicle \\
\hline FOM & - & Figure of Merit \\
\hline FS-MPC & - & MPC with Finite Control Set \\
\hline HRES & - & Hybrid Renewable Energy System \\
\hline MIMO & - & Multiple-Input Multiple-Output \\
\hline MISO & - & Multiple-Input Single-Output \\
\hline MPC & - & Model Predictive Control \\
\hline MPCC & - & Model Predictive Current Control \\
\hline MPDPC & - & Model Predictive Direct Power Control \\
\hline MPVC & - & Model Predictive Voltage Control \\
\hline NTU & - & Nanyang Technological University \\
\hline OPDC & - & Ordered Power-Distributive Control \\
\hline PCCM & - & Pseudo-Continuous-Conduction Mode \\
\hline PSC & - & Pulsating Source Cell \\
\hline PSO & - & Particle Swarm Optimization \\
\hline QRZCS & - & Quasi-Resonant Zero-Current Switching \\
\hline RES & - & Renewable Energy System \\
\hline SIDO & - & Single-Input Dual-Output \\
\hline SIMO & - & Single-Input Multiple-Output \\
\hline
\end{tabular}




$\begin{array}{lll}\text { SISO } & - & \text { Single-Input Single-Output } \\ \text { SI-DIDO } & - & \text { Single-Inductor Dual-Input Dual-Output } \\ \text { SI-MIMO } & - & \text { Single-Inductor Multiple-Input Multiple-Output } \\ \text { SI-SIDO } & - & \text { Single-Inductor Single-Input Dual-Output } \\ \text { SI-SIMO } & - & \text { Single-Inductor Single-Input Multiple-Output } \\ \text { SOC } & - & \text { State-Of-Charge } \\ \text { SPW } & - & \text { Single Primary Wind } \\ \text { ZVS } & - & \text { Zero-Voltage Switching } \\ \text { ZCS } & - & \text { Zero-Current Switching }\end{array}$




\section{CHAPTER 1 INTRODUCTION}

This chapter discusses the background of the research works in this thesis, i.e., an introduction to hybrid renewable energy systems (HRESs) and EVs including their recent developments, advantages and challenges. The research motivations, objectives, contributions and organization of the thesis are also presented.

\subsection{BACKGROUnd AND Motivation}

\subsubsection{Introduction of Hybrid Renewable Energy Systems}

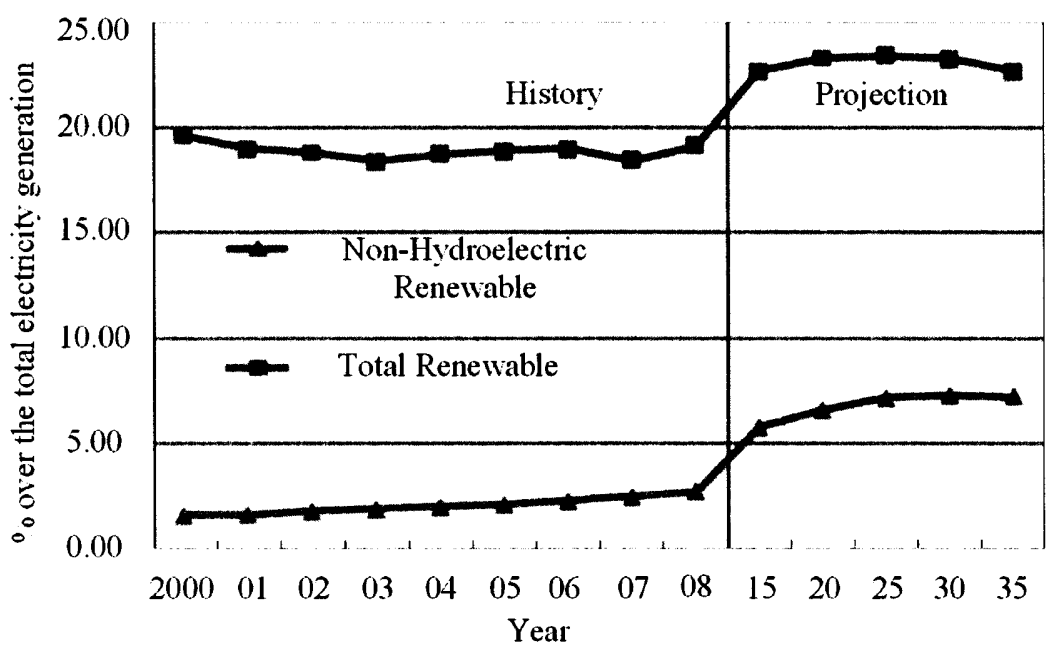

Figure 1-1: Percentage of world renewable electricity generation over the total electricity generation [5].

Nowadays, the growing global energy needs have led to excessive use of the nonrenewable fossil fuels, which further causes energy crisis and global warming [1], [2]. The energy crisis and greenhouse gas effect urge the extensive adoption of the environment-friendly renewable energy sources, such as wind, sunlight, wave and so on [3], [4]. Figure 1-1 shows the percentage of world renewable generation over the total electricity generation [5].

These renewable energy sources provide the sustainable power generation. Therefore, the governments have taken actions for the promotion of renewable energy sources including promulgation of corresponding policies and investment in research and infrastructure establishment [3], [6]-[9]. 


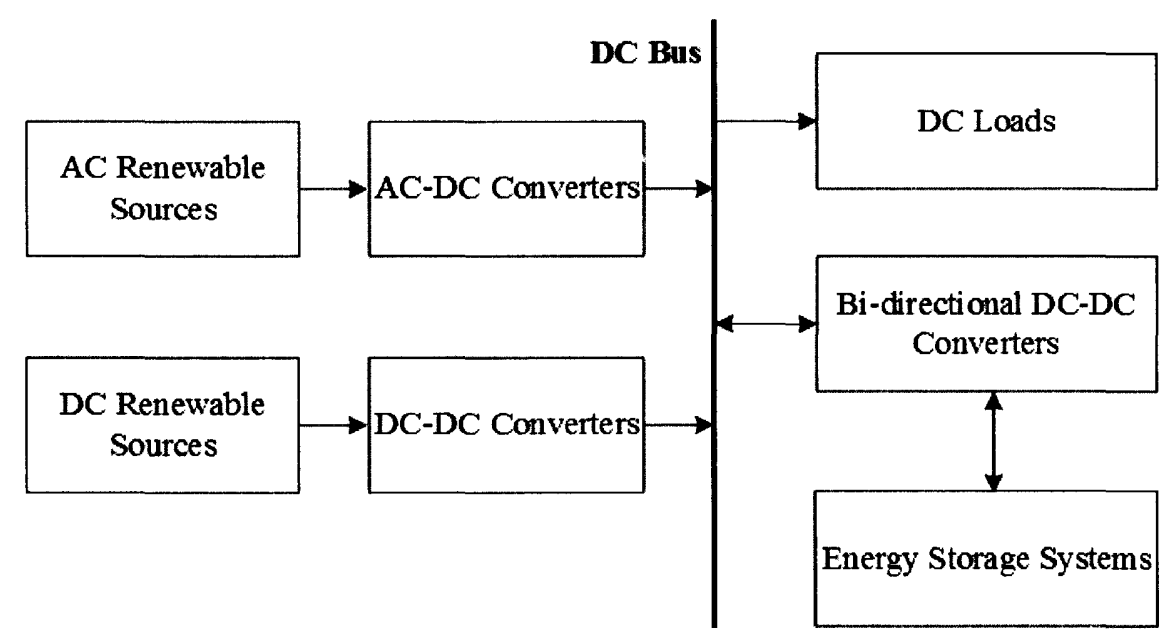

Figure 1-2: A typical HRES with DC bus [19].

Consequently, the renewable energy systems, such as solar power systems [10]-[12], wind power systems [13]-[16] and wave power systems [17], [18], have been developed and studied. However, these power generation systems using single type of renewable energy are not capable to guarantee the consistent power due to their nature features. The examples are that the solar power keeps fluctuating during the day [11], and the wind power generation is affected by the seasonal variation of wind in the system installed location [13].

HRES is one promising solution to the previous problem by integrating different types of generation sources [19]. Figure 1-2 shows a typical HRES, and it can be observed that the converters play a critical role in the HRES for energy conversion. The multiple renewable energy sources are able to help the shortages of each other so that HRESs have better performance and stability. Some practical HRESs have been built around the world such as the wind-photovoltaic-electrolyzer-fuel cell system in the United States [20] and the wind-hydrogen system in Norway [21], [22]. Unfortunately, there are some disadvantages of the HRESs, i.e., the high cost of installation and complex control [19], [23]. Recently, lots of research works have been conducted to improve the performance and overcome the disadvantages of the HRESs [24]-[33].

The assessment methods for HRESs have been proposed in [24]-[26]. In [24], the authors have conducted a small-signal stability analysis of a HRES in time domain, which consists of wind turbine generators, a photovoltaic system, a diesel engine generator and two fuel cells. Moreover, the analysis results have demonstrated that the 


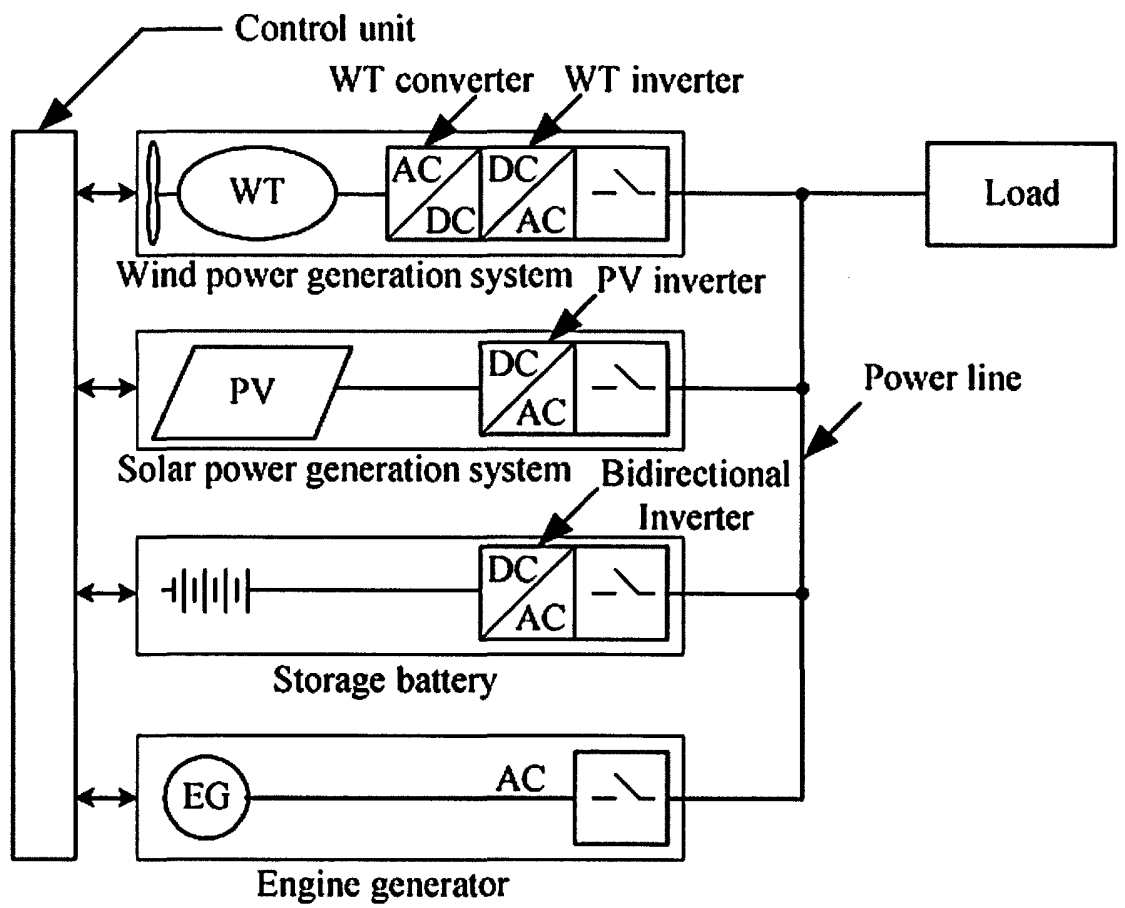

Figure 1-3: A standalone wind-solar system with dump power control [29].

proposed HRES is able to handle the variation in load power demand. A stochastic performance assessment method for a solar-wind-storage system has been developed in [26], based on which the optimization of the system size and cost can be achieved. Similarly, the optimizations of the size and capacitor allocation for HRESs have been discussed in [27], [28] respectively. These optimization methods facilitate the performance improvement of the HRESs.

The control methods have been proposed to regulate HRESs for standalone operation in [29]-[31]. In [29], a standalone wind-solar system with the dump power control has been presented as shown in Figure 1-3. The proposed method avoids the overcharging of battery and makes full usage of surplus power, so that it can extend the battery lifetime and improve the system efficiency. The authors have developed a hybrid system including off-shore wind turbine and tide turbine in [30]. Since the more stable tide power is able to compensate the fluctuation of the wind power, the combination of these two energy sources contributes to steady power output with maximum power point tracking control. An operation and control strategy for energy management in a standalone HRES has been proposed in [31]. With the proposed strategy, the optimal power utilization and continuous operation of system under different conditions can be guaranteed. 


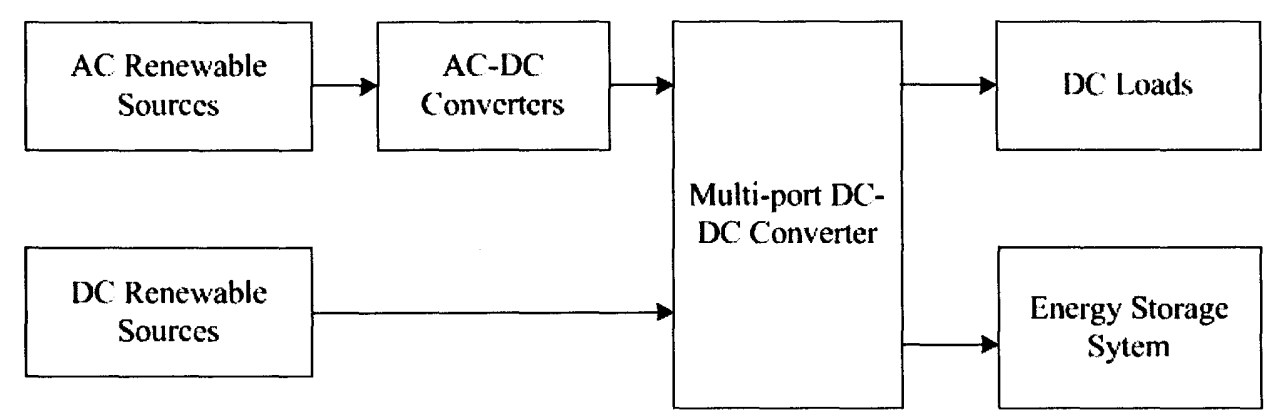

Figure 1-4: A typical HRES using multi-port DC-DC converter.

In [32], [33], the multi-port DC-DC converters have been introduced into HRESs to reduce the cost and complexity of the target system. A HRES with a multiple-input single-output (MISO) flyback converter has been studied in [32]. In [33], the authors have proposed a multiple-input multiple-output (MIMO) topology for the energy conversion in HRESs. These multi-port DC-DC converters decrease the number of components in HRESs, which further reduce the circuit size, system cost and control complexity, compared to the conventional HRESs using SISO DC-DC converters for energy conversion. A typical HRES with multi-port DC-DC converter is shown in Figure 1-4.

\subsubsection{Introduction of EVs}

The energy crisis also leads the rapid rise of gasoline price [34], [35], which is the main power source for the traditional vehicles. Moreover, the traditional vehicles release air pollutants. In the last decades, EVs have been receiving widespread attention because of their green and economic features [36]-[38]. Therefore, for the sake of environmental protection and reduction of reliance on fossil fuels, governments of many countries or areas including USA, European Union, China, Japan, etc. significantly invest in this field and promulgate corresponding policy for the promotion of EVs. For example, the Chinese government provides subsidy for EVs based on the battery capacity to encourage the usage of EVs [38].

Different from conventional fossil fuel-powered vehicles, EVs consume the electricity which is from either grid or a wide range of renewable energy sources such as solar power, wind power, tidal power, and any kind of combination of these renewable energy sources [39]-[43]. Figure 1-5 shows a EVs charging station which is powered by the standalone PV panel roof. A comprehensive review on the integration of EVs and renewable energy sources has been presented in [41]. The authors point out 
Table 1-1 Range in Miles, Battery Types and Charging Types of EVs [44], [45].

\begin{tabular}{cccccc}
\hline Brand & Nissan & Honda & Ford & BYD & Tesla \\
\hline Model & Leaf & Fit & Focus & E6 & Model S \\
\hline Range in Miles & 73 & 140 & 100 & 127 & $208-310$ \\
\hline \hline Battery Types & Lead Acid & Nickel Metal Hydride & Molten Salt & Lithium-ion & Ultracapacitor \\
\hline \hline Charging Types & Classical Charging & Fast Charging & Battery Replacement & Wireless Charging \\
\hline Advantages & Low Cost & Short Time & Short Time & $\begin{array}{c}\text { Safe } \\
\text { Convenient }\end{array}$ \\
\hline Disadvantages & Long Time & $\begin{array}{c}\text { High Cost } \\
\text { Low Efficiency }\end{array}$ & $\begin{array}{c}\text { Costly Machinery } \\
\text { Large Storage Place }\end{array}$ & Low Efficiency \\
\hline
\end{tabular}

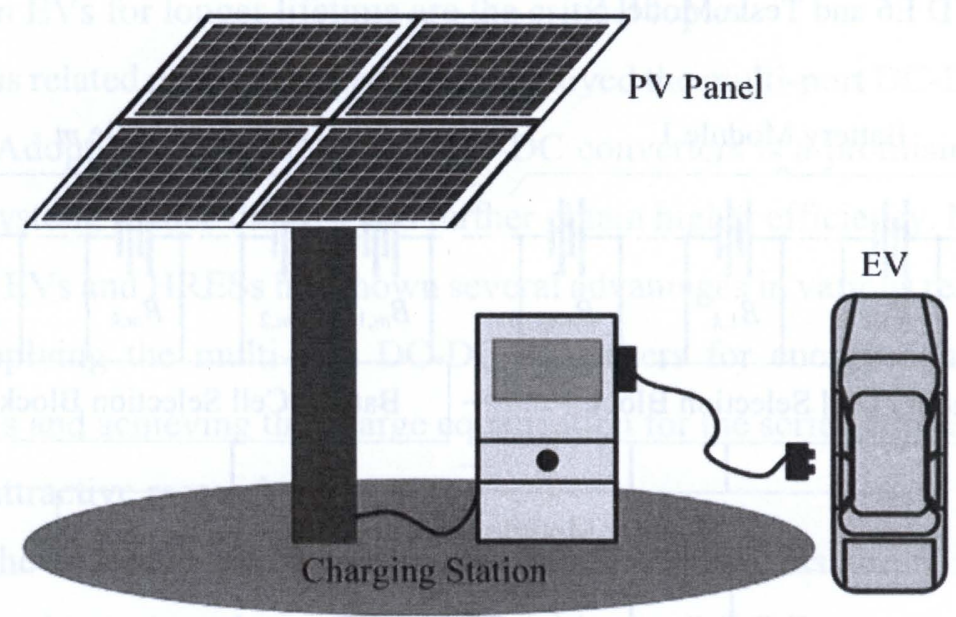

Figure 1-5: EV charging station deploying standalone PV panel on rooftop [41].

that the batteries in EVs can be utilized as the energy storage system (ESS) for the renewable energy system (RES) and grid. The ESS can absorb the redundant energy from RES or deliver power to the grid when the RES is on low power generation condition, which will relieve the penetration of RES to grid. In [42], the research results have further demonstrated that the adoption of EVs in RES and grid will improve the energy efficiency and reduce energy cost.

Due to the aforementioned advantages, EVs have a significant development recently. Some automobile companies have manufactured practical EVs, such as Nissan Leaf, Honda Fit EV, Ford Focus Electric, BYD E6 and Tesla Model S as shown in Figure 16. However, limited storage capacity of battery confines EVs' endurance ability. Most of current commercially available EVs have a short drive range under once charging as listed in Table 1-1 above. Moreover, the battery types and charging types, including the attributes, are also presented in Table 1-1. The range in miles of EVs is apparently inferior to gasoline-fueled vehicles under once refueling. However, the bright side is 


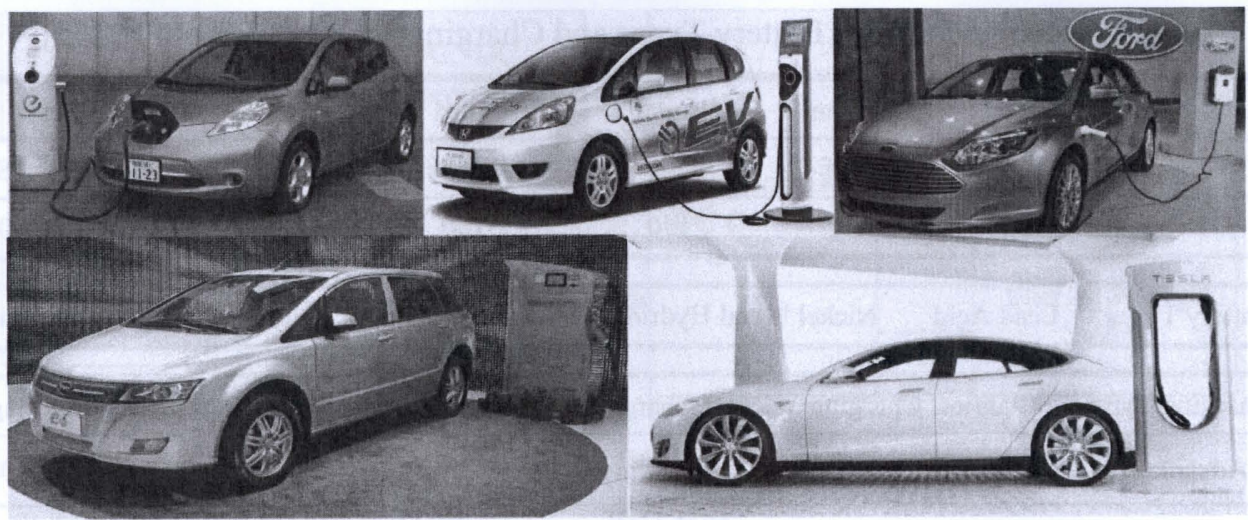

Figure 1-6: EV productions, up row: Nissan Leaf, Honda Fit EV and Ford Focus Electric; down row: BYD E6 and Tesla Model S.

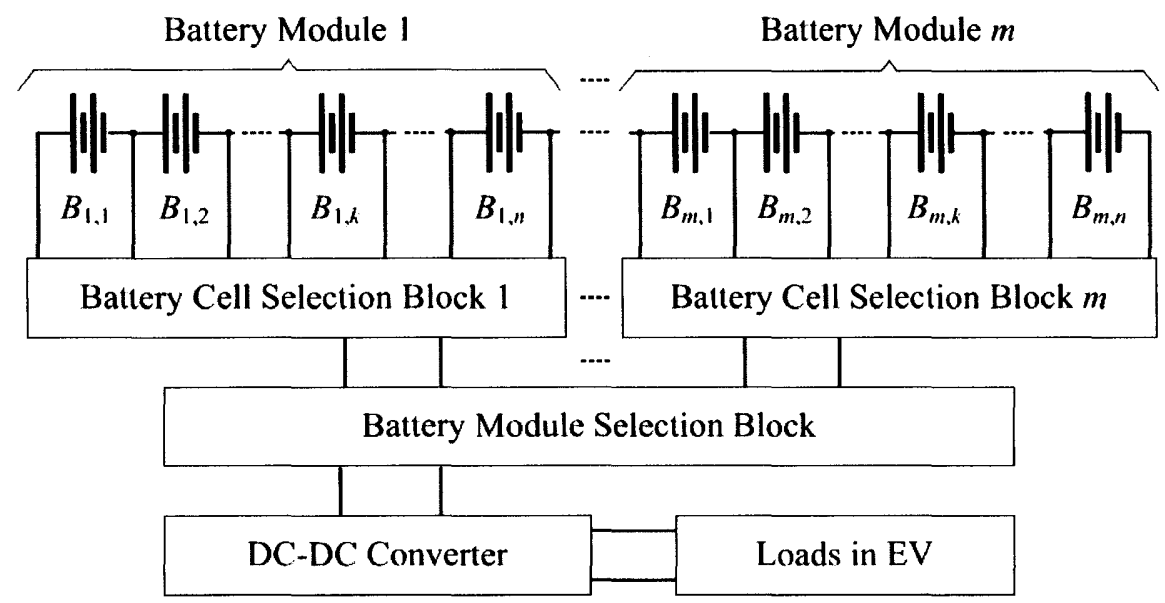

Figure 1-7: Series-connected batteries in EV [47].

that the energy cost per mile of the EVs is far less than that of the gasoline-fueled vehicles. A possible solution is increasing the battery size to obtain larger capacity for longer drive range, but it will lead to much more cost or not feasible for mechanical consideration. Consequently, repeated charging is imperative to fulfill longer mileage. Researchers investigate several types of charging technologies in [45], including conventional charging (slow charging) method, battery replacement method and wireless charging method.

Generally, the batteries are connected in series to drive the EVs [46]-[49], as shown in Figure 1-7. However, imbalance happens when the series-connected batteries are charged and discharged for too many times, which will further seriously damage battery health and shorten battery lifetime [48]. Therefore, charge equalization is a critical consideration to extend battery lifetime. In [46], [50] and [51], the authors have studied 
the effect of the charge equalization on battery life, and the experimental results have demonstrated that batteries will obtain an extension of lifetime if the charge equalization is well performed.

\subsubsection{Motivations}

The driving forces, which are from the urgent demand for reducing carbon emissions and solving the energy crisis, promote the growing research interest and investment in HRESs and EVs. It can be concluded from the previous background discussions that improving the efficiency and reducing the system complexity of HRESs, and balancing the batteries in EVs for longer lifetime are the critical topics.

The previous related research works have employed the multi-port DC-DC converters into HRESs. Adoption of the multi-port DC-DC converters is a promising solution to simplify the system, reduce the cost and further obtain higher efficiency. Moreover, the integration of EVs and HRESs has shown several advantages in various research works. Therefore, applying the multi-port DC-DC converters for energy conversion from HRESs to EVs and achieving the charge equalization for the series-connected batteries in EVs is an attractive research topic.

Based on the aforementioned review, the motivation of this thesis is to develop efficient and robust control strategies for multi-port DC-DC converters. With the proposed strategies, the multi-port DC-DC converters can be easily and efficiently applied into HRESs and EVs, and even the systems consisting of both HRESs and EVs. Furthermore, among the multi-port DC-DC converters, those using single inductor/transformer have simpler topology compared to the others. Therefore, the research works in this thesis focus on developing the control methods for multi-port DC-DC converters using single inductor/transformer in HRESs and charge equalization for series-connected batteries in EVs.

\subsection{OBJECTIVES OF THE THESIS}

As discussed previously, the multi-port DC-DC converters with single inductor/transformer are studied in this thesis. The critical problem of the single inductor/transformer topology is the cross regulation. Therefore, this thesis mainly works on solving the cross regulation problem for the multi-port DC-DC converters with single inductor/transformer topology.

The detailed objectives of this thesis are listed as follows: 
1. The first objective is to develop an output voltage regulation method for the SI-SIMO DC-DC converters to drive multiple loads, and solve the cross regulation problem. In other words, the proposed method will be effective and robust to realize the independent voltage regulation. In addition, it is able to cope with the variations in load demands and output voltage references.

2. The second objective is to work on an output current regulation method for SI-SIMO DC-DC converters for battery charging. Based on the proposed current regulation method, a charge strategy is further developed to achieve the charge equalization for series-connected batteries in EVs. Since the battery charging has CC charging stage and $\mathrm{CV}$ charging stage, the development of the corresponding current reference assignment algorithms for these two stages are also essential.

3. In order to fulfill the requirement of interfacing hybrid power sources and multiple loads in HRESs, a SI-MIMO DC-DC converter with corresponding control method is proposed as the third objective. The proposed method is supposed to have the ability to regulate the power sharing ratios of the multiple input power sources, and supply accurate power to loads by suppressing the cross regulation, which results from the single-inductor topology.

4. Flyback converter is widely used as the power supply in industrial products. The SIDO flyback converter extends the drive ability with fewer components. The fourth objective aims to develop a control method to decouple the cross regulation of the SIDO flyback converter. Consequently, the proposed method is able to regulate the dual outputs individually so that the turns ratio of the two secondary coils does not restrict the dual outputs voltage ratio.

5. In order to verify the previously mentioned methods, the simulation and hardware platforms are built including SI-SIMO DC-DC converter, SI-MIMO DC-DC converter and SIDO flyback converter. Moreover, the controllers for the hardware platforms are imperative to implement the corresponding control methods. The simulation platforms are built in the Matlab/Simulink. The hardware platforms involve PCB circuits design and DSP controller development. With these platforms, the validation of the previous proposed methods can be conducted, and two critical investigations are listed as follows: 
Steady-state operation: the proposed methods have the capability to work in steady state under different conditions, including different load demands and different power sources.

Dynamic performance: the proposed methods are able to respond to the step changes in load demands, output voltage/current references and so on, and maintain the successful operation of the target systems using the multi-port DC-DC converters.

\subsection{Contributions OF THE Thesis}

The main contributions of this thesis are summarized as follows:

1. In this thesis, the MPC strategy is applied to several types of multi-port DC-DC converters including SI-SIMO DC-DC converter, SI-MIMO DC-DC converter and SIDO flyback converter. Considering the different topologies of these three multiport DC-DC converters, the corresponding control methods are proposed based on MPC. These proposed control methods inherit the advantages of MPC over the conventional control methods, such as varying switching frequency, no modulator, on-line optimization and easy to incorporate constraints.

2. For SI-SIMO DC-DC converter, a MPVC method is developed. The proposed MPVC method is able to regulate the output voltages to follow the preset voltage references closely, which means the voltage ripples are controlled within the tolerate range. In addition, the cross regulation is significantly reduced. The state-space model, cost function and constraints of the proposed MPVC method are developed. To verify the proposed MPVC method, a SI-SIDO buck converter is built in the MATLAB/Simulink for simulation, and the corresponding hardware circuit of the SISIDO buck converter is designed and fabricated. Moreover, the MPVC method is implemented into a TI DSP controller for real-time control.

3. Similarly, a MPCC method for SI-SIMO DC-DC converter is also proposed. The proposed MPCC method is able to regulate the output currents individually and reduce the cross regulation. Furthermore, a charge equalization method for seriesconnected batteries in EVs is presented based on the MPCC method. The proposed charge equalization method is capable to charge the batteries with the proper currents based on the corresponding real-time SOCs, so that imbalanced the batteries can be eliminated with this individual charge operation. Additionally, the current reference assignment algorithms for $\mathrm{CC}$ charging stage and $\mathrm{CV}$ charging stage are proposed 
since the battery charge is split into these two stages. A simulation platform of the SI-SIMO buck converter is established in the Matlab/Simulink for the verification of the proposed charge equalization method.

4. The SI-MIMO DC-DC converter is studied to meet requirement of the multiple power sources and loads integration in HRESs. Similarly, the SI-MIMO DC-DC converter has the cross regulation problem. Therefore, MPC is also utilized. Another consideration is the regulation for power sharing ratios of the multiple-input power sources. Hence, the development of the power sharing control method is also proposed. At last, a power sharing and cross regulation suppression method is achieved for the SI-MIMO DC-DC converter. Simulation platform of a singleinductor dual-input dual-output (SI-DIDO) buck converter are designed for the performance estimation. With the guidance of the simulation, the hardware prototype of the SI-DIDO buck converter is built, and the proposed method is implemented into a dSPACE control board and a TI DSP controller for on-line control.

5. Finally, a DMPVC method for the SIDO flyback converter is proposed. The transformer in the flyback converter has one primary winding and two secondary windings. The proposed DMPVC method can regulate the two output voltages individually regardless of the turns ratio of the two secondary coils in the transformer, and suppress the cross regulation. The development of the DMPVC method is implemented systematically including the state-space models and cost function. The SIDO flyback converter is built in the MATLAB/Simulink for simulation.

\subsection{Organization OF The Thesis}

This thesis is organized into seven main chapters as follows:

\section{Chapter 1: Introduction}

This chapter provides background information of multi-port DC-DC converters for the HRESs and EVs including their advantages, challenges and recent developments. The motivations, objectives and contributions of this thesis, as well as the organization of this thesis are also discussed.

\section{Chapter 2: Literature Review}

In this chapter, a comprehensive review of the related research works on the multiport DC-DC converters, including SIMO, MISO, MIMO and flyback topologies, is 
presented. Especially, the multi-port DC-DC converters using single inductor/transformer are discussed in detail. Then, the MPC strategy, which is the critical control method adopted in this thesis, and its current applications are discussed. Finally, this chapter delivers an overall description of state-of-art charge equalization methods for the series-connected batteries in EVs.

\section{Chapter 3: Model Predictive Voltage Control for SI-SIMO DC-DC Converter}

Based on the MPC theory, the MPVC method for the SI-SIMO DC-DC converter is presented in this chapter. State-space model, cost function, constraints and priority calculation algorithm, which are the essential factors of the MPVC method, are discussed systematically. The SI-SIDO buck converter is adopted to verify the proposed MPVC method, and the corresponding simulation and experimental platforms are built with carefully-designed circuit components. A series of simulation and experimental test cases are conducted to estimate the proposed MPVC method including steady-state operation and dynamic performance. In the dynamic performance test case, load regulation and response to step change in voltage reference are studied. The simulation and experimental results are analyzed in detail. Finally, the proposed method is compared with the existing methods for the SI-SIMO DC-DC converter.

\section{Chapter 4: Model Predictive Current Control for Charging Equalization based on SI-SIMO DC-DC Converter}

A battery charge equalization method for the series-connected batteries in EVs is presented in this chapter. This method adopts the SI-SIMO DC-DC converter to realize the individual charging operation for each battery cell. In order to solve the cross regulation problem of the SI-SIMO DC-DC converter, the MPCC method is employed. Furthermore, relevant current reference assignment algorithms for CC charging and CV charging are also proposed, which provide proper current references for each battery cell. The charge equalization system with SI-SIMO buck converter and seriesconnected batteries is developed in MATLAB/Simulink. The ability of the proposed MPCC method to suppress the cross regulation is studied. Therefore, the simulation study cases of the charge equalization method under small SOC difference and large SOC difference conditions are conducted.

Chapter 5: Power Sharing and Cross Regulation Suppression Method for SIMIMO DC-DC Converter 
In this chapter, the SI-MIMO DC-DC converter is studied. The regulation of this converter involves input powers and output voltages, i.e., the power sharing and cross regulation suppression respectively. The power sharing and cross regulation suppression method is presented in detail including MPC method, power sharing method and time-multiplexing method. A SI-DIDO buck converter is designed to verify the proposed method. The circuit components of the converter are carefully selected. The steady-state operation and dynamic performance of the proposed power sharing and cross regulation method are conducted in the simulation and experimental platforms. The dynamic performance study cases consist of line regulation, load regulation, response to the input current reference step change and response to the output voltage reference step change.

\section{Chapter 6: Dynamic Model Predictive Voltage Control for SIDO Flyback Converter}

The MPC strategy is further applied into the SIDO flyback converter in this chapter. Cross regulation is the critical problem because the SIDO flyback converter adopts the transformer with single primary coil and dual secondary coils. A DMPVC method for the SIDO flyback converter is proposed to reduce the cross regulation. The state-space models of the SIDO flyback converter in different conditions for the DMPVC method are formulated, as well as the corresponding cost function. In order to verify the performance of the proposed DMPVC method, the steady-state operations including buck-buck mode, buck-boost mode and boost-boost mode are simulated. Furthermore, the mode transformations, i.e., transformation from buck-buck mode to buck-boost mode and transformation from buck-boost mode to boost-boost mode, are also studied. The simulation results are analyzed in detail.

\section{Chapter 7: Conclusions and Future Works}

Based on the previous discussions, an overall conclusion of this thesis is drawn in this chapter. Moreover, the recommended future works are also discussed. 


\section{CHAPTER 2 LITERATURE REVIEW}

\subsection{Multi-Port DC-DC CONVERTER}

As discussed previously, HRESs with multiple-input power sources have been developed because of their advantages including better reliability, longer durability and higher efficiency compared to the systems with single renewable energy [43]. In order to interface the various renewable energy sources and loads, the multi-port DC-DC converters have been designed in HRESs with fewer components, smaller size and less cost resulting from their topologies [52], [53]. The multi-port DC-DC converters are categorized as MISO DC-DC converters, SIMO DC-DC converters and MIMO DCDC converters.

In this thesis, the research works focus on the multi-port DC-DC converters with single-inductor/transformer topology, which is able to further simplify the architecture of converters and reduce the number of components. For the SI-MISO DC-DC converters, the critical consideration is to regulate the power sharing ratios of multiple power sources. For the SI-SIMO DC-DC converters, cross regulation problem is the main issue to be solved. Hence, for the SI-MIMO DC-DC converters, power sharing and cross regulation suppression should be taken into consideration simultaneously.

\subsubsection{MISO DC-DC Converter}

Many research works on topologies and control methods for the MISO DC-DC converters in HRESs have been conducted [54]-[64]. The main purpose of these research works is to regulate the power flows from the different power sources, i.e., power sharing control.

In [54], a multiple-input DC-DC converter using single inductor has been proposed for hybrid energy conversion. The proposed converter is able to operate as buck, boost or buck-boost converter in discontinuous-conduction-mode (DCM). Moreover, the proposed converter has the capability to realize the bi-directional energy conversion. A multiple-input boost converter to harvest energy from low-power energy sources has been presented in [55] as shown in Figure 2-1. Since the proposed converter adopts the single inductor topology, an automatic digital control method based on timemultiplexing operation is developed. Moreover, the optimal load resistance condition 


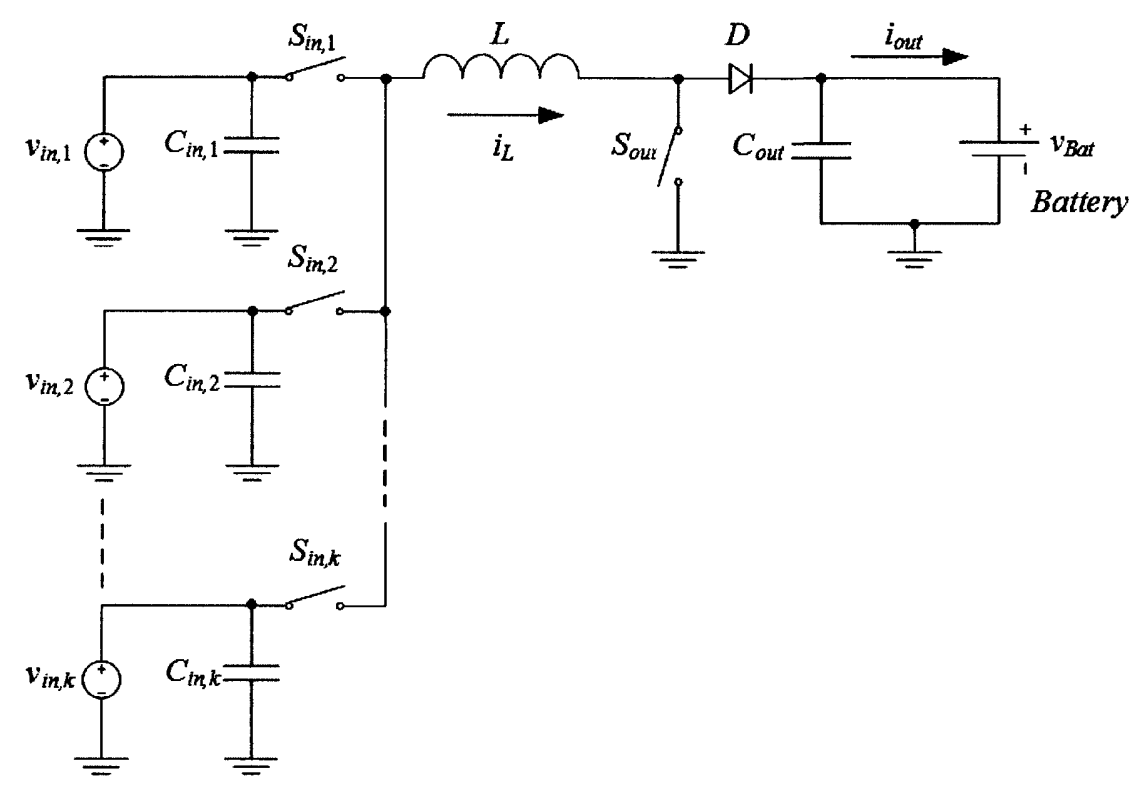

Figure 2-1: The architecture of a typical MISO boost converter [55].

varies for maximum power extraction because of switching of input power sources. To obtain the maximum power, an adaptive method based on pulse counting control is proposed so that the equivalent resistance of the converter is maintained on the maximum power extraction condition.

The researchers have designed a single primary wind (SPW) multiple-input flyback converter in [56]. Different from the traditional multiple-input flyback converters, the proposed converter contributes to simpler structure, easier manufacturing and less leakage by incorporating the SPW transformer to achieve multiple power sources regulation. Moreover, the SPW topology enables the simultaneous energy transfer from the multiple power sources rather than the time-multiplexing way of the traditional multiple-wind topology, which means it can deliver more power to load in a given instant.

Dual-input converters using H-bridge cells have been fully studied in [57]. The dualinput converters include four topologies such as buck-buck, buck-buckboost, buckboost-buckboost and boost-buck-boost. The corresponding small-signal models are developed using state-space averaging approach. By controlling the switches of the H-bridge cells, the arbitrary power sharing ratios can be achieved. Meanwhile, the output voltage is maintained.

In [58], the authors have developed a three-input boost converter for a hybrid photovoltaic/fuel cell/battery power system. The proposed converter has the capability 
to incorporate two unidirectional input power sources and a bidirectional energy storage element. Duty cycles for power switches are regulated dynamically and independently to adjust the power flows from the input sources and deliver the proper power to the load. Meanwhile, the proposed converter is able to extract energy from the input power sources to the load individually or simultaneously. In order to design the control system, the small-signal models of the proposed converter are derived for three operation modes. In summary, the proposed converter has four main advantages including economic structure, reliable stability, dynamic input power sharing ratios and large range of output voltage.

A modified time-multiplexing switching technique has been proposed for a MISO buckboost converter in [59]. The authors have defined a concept of common duty ratio (CDR), based on which the duty cycles of power switches are calculated by multiplying the CDR with corresponding integers. Hence, there is only one control variable so that the control complexity is simplified. In detail, the MISO buck-boost converter can be equivalently regarded as a SISO buckboost converter controlled by a single proportionintegration (PI) controller.

In [60], the researchers have presented a pulsating source cell (PSC) concept and corresponding controller based on the small-signal model for a MISO converter. The proposed converter is able to absorb energy from different series-connected energy sources regardless of the symmetric or asymmetric source voltage magnitudes. Moreover, the proposed method and topology allow the individual and simultaneous regulation of all the series-connected energy sources, and even bi-directional energy conversion for energy storage.

A Lyapunov-based nonlinear power sharing control method has been proposed for the dual-input buckboost-buckboost converter in [61]. The proposed control method is independent from the operating point of the dual-input buckboost-buckboost converter. With the nonlinear power sharing control method, line regulation, load regulation and dynamic power sharing ratios from input power sources can be successfully performed, and the stability of the proposed converter is maintained.

In [62], a new topology of dual-input interleaved converter has been designed. In order to achieve the individual or simultaneous energy conversion from two renewable power sources to loads and the mutual charge between the power sources, four operational states have been developed, which are a single-input step-up state, a dualinput step-up state, a dual-output step-down state, and a dual power source step-up/step- 


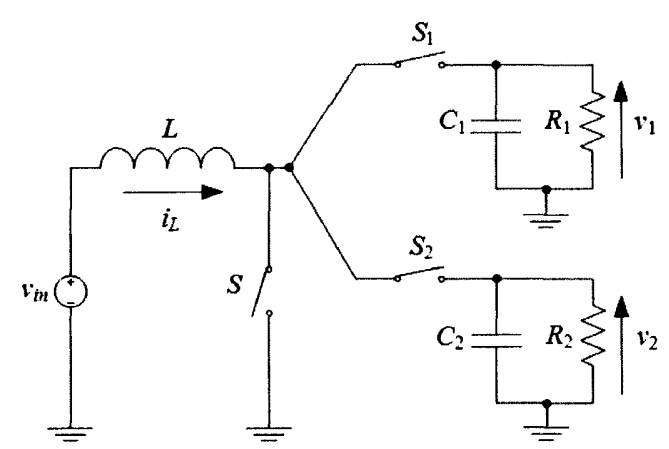

(a)

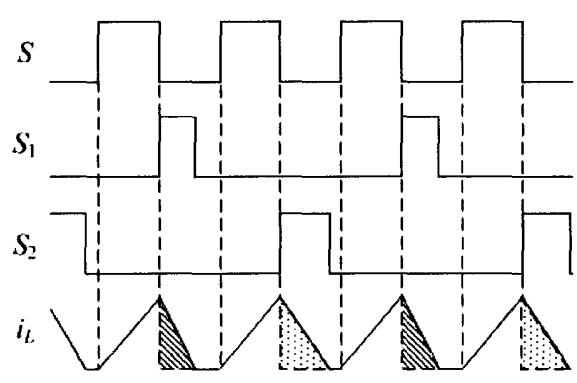

(b)

Figure 2-2: The proposed SI-SDIO boost converter [65]. (a) Architecture of the SI-SIDO boost converter. (b) Timing diagram of the SI-SIDO boost converter.

down state. In these four states, the proposed interleaved structure can reduce the ripples of the input currents and the output voltage and relieve the current stresses upon the power switches. Furthermore, the dual-input interleaved converter is regulated in synchronous conduction mode so that the zero-voltage switching (ZVS) for turning on all switches can be obtained for high-efficiency operation.

In [63], a flyback-based MISO converter to charge batteries and ultracapacitors in EVs has been developed, consisting of bi-directional converter part, cell voltage equalizers part and regenerative unit part. Therefore, the proposed converter enables the bidirectional energy flow between the fuel cells and ultracapacitors. In addition, the cell voltage equalizers embedded in the proposed converter are able to guarantee equal charge or discharge of the batteries or the ultracapacitors. As discussed previously, the charge and discharge equalization are beneficial for the health and lifetime extension of the batteries.

In [64], the authors have presented a steady-state analysis tool of time-sharing switching scheme for general MISO converters in continuous-conduction-mode (CCM) and DCM. The proposed tool can describe the influences of the critical parameters, including modulation type, conduction sequence, input voltage, load value, duty cycle and switching frequency, in the MISO converters.

\subsubsection{SIMO DC-DC Converter}

Based on aforementioned discussion, the critical issue of the SIMO DC-DC converter using single inductor is the mutual influence among the multiple loads, i.e., cross regulation problem. In [65]-[75], researchers have developed various control methods to regulate the SIMO DC-DC converter and reduce the cross regulation. 


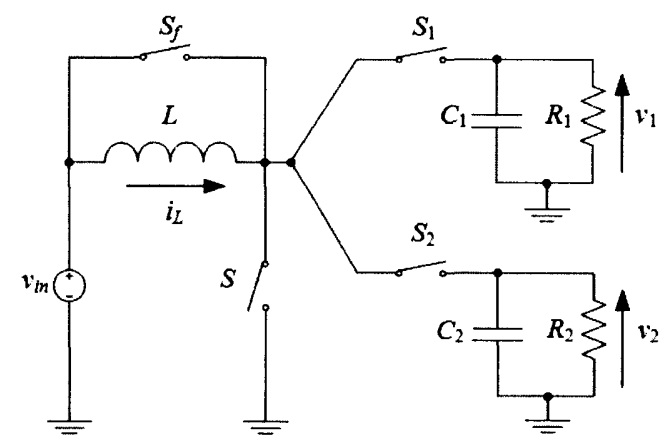

(a)

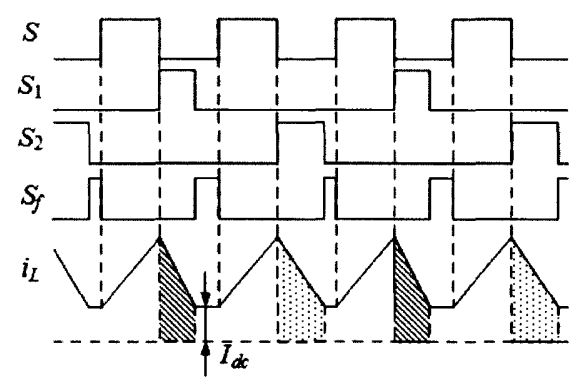

(b)

Figure 2-3: The proposed SI-SDIO boost converter with a freewheeling switch [66]. (a) Architecture of the SI-SIDO boost converter with a freewheeling switch. (b) Timing diagram of SI-SIDO boost converter.

In [65], the time-multiplexing method has been developed to control the SI-SIDO DC-DC converter for solving the cross regulation problem. The corresponding converter and regulation waveforms are shown in Figure 2-2. It can be seen that the proposed converter works in the DCM by rotating the loads only after the inductor current drops to zero. Therefore, there is no residual energy in the shared inductor so that the mutual influence between the two loads is completely avoided. However, it also can be observed that the proposed SI-SIDO DC-DC converter experiences a large peak current through the shared inductor in DCM, especially when the heavy load condition happens.

To solve the cross regulation problem and reduce the high peak current through inductor in [65], the authors have further developed the pseudo-continuous-conduction mode (PCCM) method in [66]. To perform the PCCM operation, a freewheeling switch is added to the two terminals of the shared inductor as shown in Figure 2-3(a), and the corresponding waveforms are shown in Figure 2-3(b). When the inductor current is reduced to the minimum value of $I_{d c}$, the proposed method will turn on the extra freewheeling switch and turn off the other switches so that the minimum value of $I_{d c}$ is maintained. Hence, the DCM is avoided and the peak current through the shared inductor is reduced. However, this freewheeling switch leads to considerable power loss, which is even larger when the voltage outputs are unbalanced. Consequently, the authors have improved the PCCM method in [67], [68], i.e., the adaptive PCCM method. The proposed method is able to dynamically adjust the minimum value of $I_{d c}$ for optimal 
operation of the freewheeling switch, which can significantly reduce the power loss in [66].

A novel ordered power-distributive control (OPDC) method has been proposed in [69]. The researchers have employed high speed voltage comparators to adjust switching duty cycles of the heavy load branch dynamically to meet its demand. In detail, there are three comparators for the first three output voltage regulation. For the fourth voltage regulation, a PI controller is adopted. With the OPDC method, the multiple output voltages can be regulated to follow the voltage references closely However, this method may fail when most branches confront heavy load condition.

A multi-loop method for SI-SIMO buck converter has been presented in [70]. The charge control is adopted for the inner loops to regulate the multiple-output voltages and the average current mode control is utilized for the external loop so that the inductor current can be regulated for CCM operation. In order to perform the loop compensation the proposed multi-loop method requires several external components.

A digital control method based on separate regulation for common-mode anc differential-mode has been proposed in [71]. In the common-mode, the performanc of response to asymmetrical load changes is improved by the nonlinear evaluation o the common-mode voltage. In the differential-mode, the dependence of differential mode loop gain on inductor current is eliminated by the nonlinear gain compensation Therefore, the proposed digital control method is able to suppress the cross regulation in CCM, and it is also easy to be extended for driving arbitrary number of output loads However, due to the voltage of the last $N$ th branch being linearly dependent on th voltages of the other $(N-1)$ branches, the proposed method may lead to failure in voltag supply of the $N$ th branch.

The researchers have developed a dual-mode control method for SI-SIDO buc converter to solve the cross regulation in [72]. The two modes consist of pulse-widt modulation (PWM) mode for light load condition and pulse-frequency modulatio (PFM) mode for heavy load condition. The mode switching can be automaticall conducted according to a duty-cycle-based load current estimation method. A flyin capacitor across the two loads is employed in the proposed converter to reduce th voltage ripples, and eliminate the voltage spike caused by the fast change of inductr current. However, the flying capacitor leads to interaction between the two outpu which is adverse for cross regulation suppression. 
In [73], the authors have developed a predictive digital current control method. A series of linear equations are deduced to describe the relationships of output voltages, output current, inductor current and the duty cycles for power switches. Consequently, the duty cycles can be calculated on-line based on the targeted references and real-time data, which are captured from the SI-SIMO DC-DC converter. Hence, the optimal duty cycles are applied to the power switches, and the output voltages are controlled to follow the voltage references, i.e., the cross regulation is significantly reduced. However, the limitation of the proposed method is that the linear equations work only when the inductor current is larger than a certain threshold.

In [74], a cross-derivative state feedback strategy has been proposed. The authors conduct the mode-by-mode averaging of the inductor current to achieve a currentripple-based small-signal model, and it can describe the SI-SIMO DC-DC converter behavior. This small-signal model is employed to develop the cross-derivative state feedback method, which can minimize the cross regulation and guarantee the stability of the converter. However, the method is designed with the pole and zero placements around a specific operating point. Therefore, the performance may be influenced by the variations of the input voltage and components.

In [75], the authors have proposed a digital multivariable controller design methodology for the SI-SIDO buck converter. In the proposed multivariable control method, a number of operating points along with the variations in the circuit parameters and input voltage are taken into the consideration. Hence, the proposed controller is able to satisfy the load demands, and decouple the output voltages simultaneously. The limitation of the proposed method is that the output voltage of one channel is always less than the other due to the topology configuration.

An adaptive current compensation (ACC) method has been proposed for a hybrid SIMO DC-DC converter in [76]. The step-up and step-down load transient responses of the converter are significantly enhanced based on the proposed ACC method, and this performance improvement is achieved without advanced control or high switching frequency. Moreover, the linear regulators, including an error detector, a transient accelerator and a power transistor, are adopted for all sub-converters. With the help of the linear regulators, the cross regulation is reduced by minimizing the interactive duty ratio change between the switching sub-converters.

In [77], SI-SIDO buck-boost power factor correction converter has been proposed. By multiplexing the shared single inductor, each output voltage can be regulated 
independently so that the cross regulation problem can be solved. The proposed converter has the advantages such as low cost, small size, light weight and high power conversion efficiency. However, the converter works in critical conduction mode, which means the peak current on the inductor is high when the load is heavy.

\subsubsection{SIMO Flyback Converter}

The flyback converters have several advantages over the conventional converters using inductor. The transformer in flyback converter is able to isolate the input power source and load. Moreover, the turns ratio of the transformer contributes to the buckboost operation and a larger voltage gain range. Therefore, the applications of flyback converter involve electric vehicles [78], photovoltaic system [79], [80], LED lighting system [81] and solid-state lamp [82] and daily electronic devices [83] including phone charger and PC power supply. To meet the multiple-voltage demands, SIMO flyback converter has been developed. Several research works for different types of SIMO flyback converters and corresponding control methods have been conducted in [84][90]. Since the primary wind is shared by the multiple secondary windings, the cross regulation exists in the SIMO flyback converters [84], [85]. In [85], the analytical model of SIMO flyback converter has been built to study the cross regulation. Theoretical and experimental investigations have been conducted to determine the crucial factors that affect cross regulation in a multiple-output flyback converter. The results demonstrate that the corresponding critical factors are primary leakage inductance, secondary leakage inductances and magnetizing inductance. Furthermore, the larger values of these factors will enhance the cross regulation. This feature of the SIMO flyback converter has been utilized to simplify control complexity [86], [87]. In other words, only one output voltage is controlled and the other output voltage is automatically regulated by the cross regulation.

In [86], a flyback converter using multiple-coupled inductor, which results in good cross regulation for low output voltages and high power density, has been developed. The two output voltages relationship is determined by the turns ratio of the two secondary windings. With multiple-couple inductor, the proposed converter can obtain a smaller current ripple, and reduce the power losses resulting from current stress and interconnection among the magnetic components.

The authors have presented a magnetic amplifier (Magamp) postregulator based on time-sharing control strategy for a multiple-output $L L C$ series resonant converter in 
[87]. The $L L C$ series resonant converter structure is realized by a transformer and there is no output-filter inductor on the secondary side, so that the proposed converter can be regarded as a flyback converter. Moreover, a frequency modulator is applied to the $L L C$ series resonant converter. Compared to conventional flyback converter, the proposed topology has three advantages, including no reverse-recovery resetting effect, better effect from application of dead time, and achievements of ZVS for primary side power switches and zero-current switching (ZCS) for secondary side power switches. Similarly, the ratio of two output voltages is fixed by the turns ratio of two secondary windings, which is one in the hardware prototype. Hence, the proposed converter only can provide two same output voltages.

In [88], the multiple outputs have been realized using a flyback converter integrated with a full-bridge DC-DC converter. The full-bridge DC-DC converter is able to achieve ZVS of the power switches, and the flyback converter works in standby mode. Although there is no cross regulation due to only one secondary winding is adopted, the ratio of the two output voltages is determined by the turns ratio.

A multiple-output converter using parallel-series-connected transformer has been discussed in [89]. The proposed converter adopts an asymmetrical PWM half-bridge flyback topology, and the output stages are connected serially. Therefore, only a simple single-output regulation is required to control the multiple-output voltages. The first harmonic approximation analysis approach is employed to determine the optimal operation frequency in order to obtain higher efficiency.

In [90], a half-bridge flyback converter with an integrated buck converter has been developed to achieve multiple-output voltages. The main output is obtained by the halfbridge flyback converter and the auxiliary output is from the buck converter. Therefore, the output voltages are isolated, which means the cross regulation among the outputs does not happen anymore. To achieve the simple structure, lower transformer size, and ZVS of all the switches the buck converter is integrated with the secondary flyback rectifier.

It can be seen that most of the previous works cannot regulate the multiple-output voltages of the SIMO flyback converter independently. In other words, the relationship of output voltages is restricted by the turns ratio of the secondary windings and duty cycles of control signals. These works take advantage of the cross regulation to realize the multiple-output regulation and achieve the simplification of the controller. In this thesis, the research works try to decouple the cross regulation and regulate the multiple- 
output voltages individually, and inherit the features of input-output isolation and large voltage range from the flyback topology.

Recently, the modeling control methods have been applied to regulate the flyback converters [91], [92]. In [91], a fourth order model has been built to control an interleaved flyback module-integrated converter. In [92], dynamic models of different modes for a parallel boost-flyback-flyback converter have been built to maintain stable output voltage. Since the methods are derived from the models which can accurately describe the flyback converters, these modeling control methods provide a better regulation performance. In this thesis, a more formal modeling control method, i.e., MPC, has been applied into the SIMO flyback converter and it can achieve the individual regulation of the multiple-output voltages.

\subsubsection{MIMO DC-DC Converter}

The MIMO DC-DC converters have drawn more and more research attentions because the MIMO DC-DC converters incorporate the advantages of the MISO DCDC converters and the SIMO DC-DC converters. Several research works for MIMO DC-DC converters have been conducted in [93]-[99].

A SI-MIMO DC-DC converter, which is applied to drive the DC devices in EVs, has been presented in [93]. The proposed converter is able to independently convert arbitrary sources to arbitrary loads using a shared inductor, which avoids bulky circuit size, saves cost and improves the system efficiency. Moreover, the proposed converter can work in CCM and DCM to provide different output voltages.

In [94], the authors have developed a MIMO DC-DC converter using multiple windings on a common transformer. The adoption of the transformer core without a centralized storage capacitor makes the proposed converter achieve the isolation of input ports and output ports. In order to regulate the proposed MIMO DC-DC converter, a PWM control method is developed, which is able to realize the average current mode control for the inter-port bidirectional power flow. Compared to the case of isolated full bridge topologies, the proposed MIMO DC-DC converter has the advantage of snubber less operation because of the leakage inductance in series with the external inductor in all stages of operation.

In [95]-[97], a comprehensive and systematic discussion on the SI-MIMO DC-DC converters has been presented. The proposed converters can interface arbitrary input energy sources and passive loads under CCM and DCM. The power budgeting between 


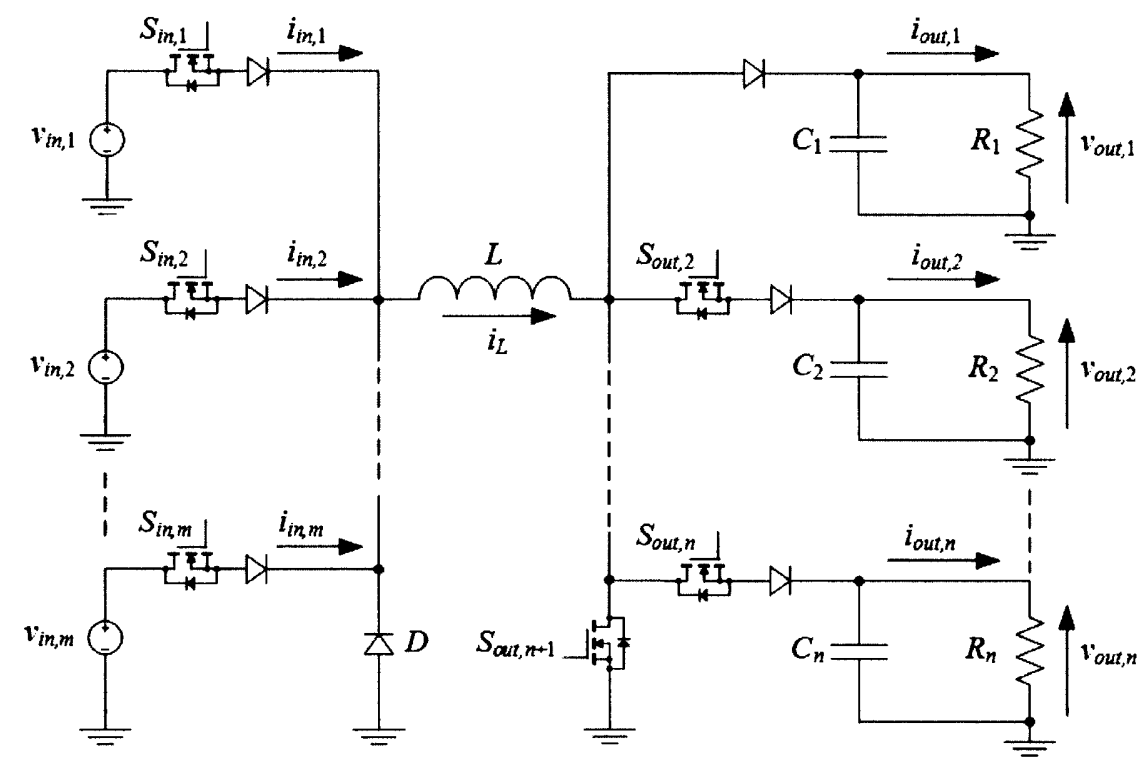

Figure 2-4: The architecture of the SI-MIMO DC-DC converter [95], [96].

input energy sources can be achieved by individually regulating the input powers delivered by different energy sources. By controlling the control signals of the switches and sensing the current through inductor, the input and output currents can be obtained without any additional current sensors for measurement. Therefore, the single inductor topology can simplify the current sensing, which further reduces the complexity and cost of the proposed converter. Moreover, the multiple-output voltages can be independently regulated in buck or boost operational mode.

In [95], [96], the proposed converter employs the parallel-output configuration as shown in Figure 2-4. The operational principles, steady-state characterizations in CCM and DCM, and the power budgeting capability of the proposed converter have been analyzed in detail. Since the shared single inductor is the critical component in proposed SI-MIMO DC-DC converter, the design of the inductor is discussed including inductor rating and inductor size in [95]. In [97], the multiple-variable feedback control methods have been proposed for the SI-MIMO DC-DC converter using series-output configuration. Besides the similar analysis of operational principles, steady-state characterizations in CCM and DCM, and the power budgeting capability, the authors also conduct the modeling of power loss and efficiency, and sensitivity analysis in [97].

In [98], an extendable MIMO boost converter has been proposed, and it consists of two bidirectional ports for output load and battery storage in the central part, and the arbitrary number of unidirectional input ports to interface different input DC sources. 
Furthermore, the proposed MIMO boost converter is able to achieve both DC-DC and DC-AC conversions without output filters. The authors have developed a poleplacement control strategy based on integral state feedback to regulate the proposed converter. In order to deploy the control method, several current regulator loops for input DC sources and two voltage regulator loops for output voltages are adopted. However, the proposed converter uses too many inductors for interfacing the input DC sources resulting in large size and electromagnetic interference problem compared to single-inductor topology.

A SI-DIDO converter has been proposed for harvesting energy from the solar system in [99], and a PWM method with corresponding control circuit has been developed to realize the output voltage regulation and the maximum power point tracking. There are two operation modes, i.e., heavy load mode and light load mode. In heavy load mode (the PV cells output power is insufficient for the load), the proposed converter regulates both the PV cells and the rechargeable batteries to supply the load together. In light load mode (the PV cells output power is larger than the load requirement), the proposed converter is able to charge the rechargeable battery using the surplus PV cells energy. Therefore, the proposed SI-DIDO converter has an efficient capability to interface the PV cells, rechargeable batteries and multiple loads.

\subsection{Charging Equalization}

Battery charge equalization methods have been classified as either passive or active. A comprehensive comparison of the advantages and disadvantages of these methods has been discussed in [100]. Most existing methods are based on the individual charging concept. In this concept, the charging of each battery cell is individually operated on its SOC.

In [101]-[103], the authors have presented the individual charge to balance the seriesconnected battery cells, which are allocated with the distributed cell equalizers. The cell equalizer is designed with a number of inductors, capacitors, diodes and power switches. In [101], the cell equalizer consists of a bidirectional DC-DC converter and an energy transferring capacitor. A series of equations have been deduced to describe the behavior of the cell equalizer, based on which a fuzzy-logic-controlled strategy within a safe equalizing region has been developed for cell equalizer control. The proposed control strategy is able to achieve the rapid cell voltage balancing. Furthermore, the guarantee of safe equalizing operation optimizes the equalization time 
and the total charging and discharging capacity of the battery string. The bi-directional DC-DC converter is also adopted as the cell equalizer in [102], and the quasi-resonant ZCS (QRZCS) technique is applied to control the proposed cell equalizer. Compared to the cell equalizer without QRZCS, the proposed equalizer has the capability to reduce switching power loss and improve the energy transfer efficiency. In [103], a modified charge equalizer based on buck-boost converter topology has been proposed with an optimal charge equalization algorithm. In the optimal charge equalization algorithm, the initial voltage and the battery capacity are utilized to regulate the charge equalization process rather than the dynamic voltage during the charge equalization phase, which contributes to the capability of excellent battery reaction inertia rejection of the cell equalizer. Hence, the optimal charge equalization algorithm shortens the charge equalization time compared to the conventional algorithms. Moreover, an activated cell equalizer duty ratio is set at the upper limits to improve the speed of charge equalization.

Bi-directional DC-DC converter has been adopted for parallel operation of parallelconnected battery cells in [104], [105]. The battery power module (BPM) concept is proposed by the authors, which includes the battery cell and bidirectional converter. With these BPMs, the charge or discharge current for each battery cell can be independently controlled based on the corresponding SOC and voltage. Therefore, the imbalance never happens. Nevertheless, the proposed BPM concept and parallel operation have two main disadvantages. The first one is that BPM concept involves lots of power converters so that the system has large circuit size and high cost. The other one is that the total conversion efficiency in parallel operation may be low, and the situation is even worse when the converter in the BPM works in boost mode.

A modularized charge equalization converter has been proposed for series-connected batteries in [106], which achieves three main advantages inducing low voltage stress for all electronic devices, practical and simple implementation, and fast charge equalization. As the higher power rating leads to the shorter charge equalization time, a design guide has been proposed to describe the relation of power rating and charge equalization time and then determine the optimal power rating. The authors also optimize the design of the charge equalization converter, which is only formed by one power switch and an isolated gate driver. Hence, the proposed equalizer reduces the size and cost of circuit compared to complete converters adopted in [101], [102], [104] and [105]. 


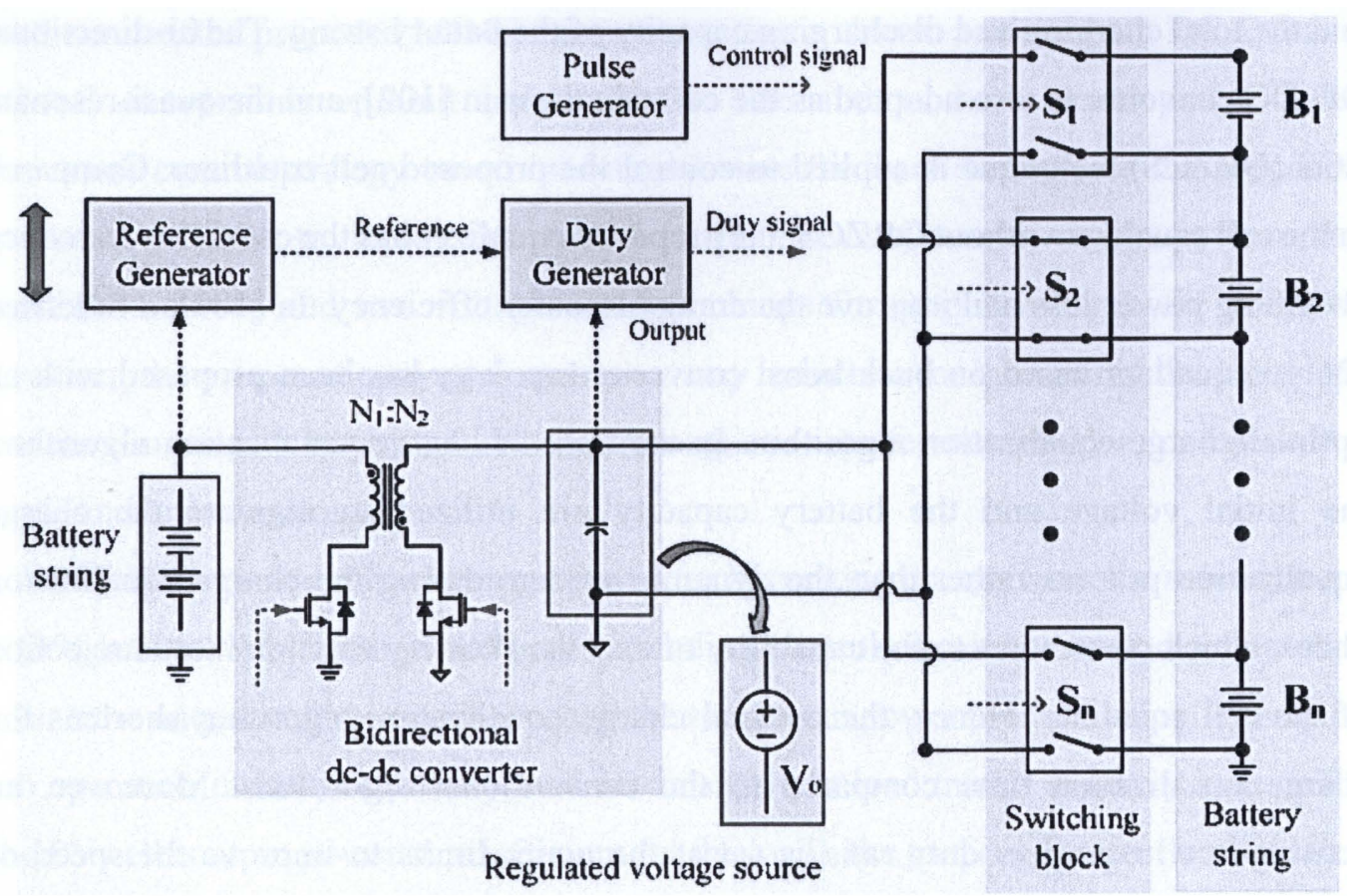

Figure 2-5: Automatic charge equalization circuit based on regulated voltage source for series connected lithium-ion batteries [108].

Based on the work in [106], a two-stage charge equalizer has been further developed in [107]. The charge equalizer consists of a DC-DC converter and cell selection switches, and the DC-DC converter is shared by the batteries. In the practical operation, the individual cell charge or discharge is realized by the cell selection switches, which provide the path between the shared DC-DC converter and the unbalanced battery cell. Compared to the equalizer in [106], the proposed configuration further simplifies the circuit topology, and reduces the corresponding cost. Another advantage of the twostage topology is that it can relieve the voltage stress of the proposed charge equalizer.

The researchers have developed an automatic charge equalizer in [108], as shown in Figure 2-5. The proposed equalizer is a bi-directional flyback converter. Different from the normal flyback converter, the diode is replaced by a MOSFET which makes the flyback converter have a symmetrical and bi-directional structure. Moreover, the body diode of one MOSFET is utilized to achieve the function of block power when the other MOSFET is turned on. The output voltage is fast regulated to the average voltage of the battery cells based on output voltage feedback control strategy. The battery cells and the bi-directional converter are connected by the switching blocks, and the connection is conducted periodically. Therefore, charge equalization can be automatically achieved because the voltages of all battery cells are always equal to the 
average value. The reference voltage modulation for a large equalization current and advanced skip mode shorten the equalization time.

In [109], the transformer is employed as the cell equalizer to convert the energy from charger or discharger to battery cells based on the corresponding terminal voltages to achieve charge equalization. The proposed topology solves the inrush current problem, and it can realize the SOC balancing and voltage balancing simultaneously with less power switches compared to the previous methods. Additionally, the proposed cell contributes to higher balancing efficiency and more compact circuit size compared with inductor-based type balancing. As a result, the proposed transformer-based cell equalizer has a good expansibility for any type of switching-converter-topology charger or discharger.

An active battery cell balancing circuit with high efficiency for series-connected battery cells has been designed in [110]. The main component in the proposed balancing circuit is a transformer, which has one primary winding and multiple secondary windings. The number of the secondary windings is equal to the battery cells. Other components are a diode and bi-directional switches. The soft switching operation is employed so that the proposed balancing circuit has the high equalization efficiency.

In [111], the authors have proposed a battery cell equalizer based on the interleaved active clamp flyback converter, and the interleaved topology is able to suppress the ripple of output current. A resonant circuit is adopted to replace the general snubber for power switches to reduce the power loss. To avoid the power loss in hard switching, ZVS is conducted by the optimal selection of switching frequency and duty cycle. In addition, the switch voltage stress is reduced due to that the surge energy recycled from the leakage inductance is absorbed by the clamp capacitor.

\subsection{Model Predictive Control}

In this thesis, the MPC method plays the critical role to regulate the multi-port DCDC converters including SI-SIMO DC-DC converter, SI-MIMO DC-DC converter and SIMO flyback converter. The comprehensive survey of the MPC method has been presented in [112], [113], and the characteristics of the MPC method are discussed in detail. Particularly, the MPC with finite control set (FS-MPC) is suitable and practical for implementation on modern digital processors.

A general control diagram of FS-MPC for power converters is shown in Figure 2-6. Generally, the FS-MPC method requires a state-space model which describes the target 


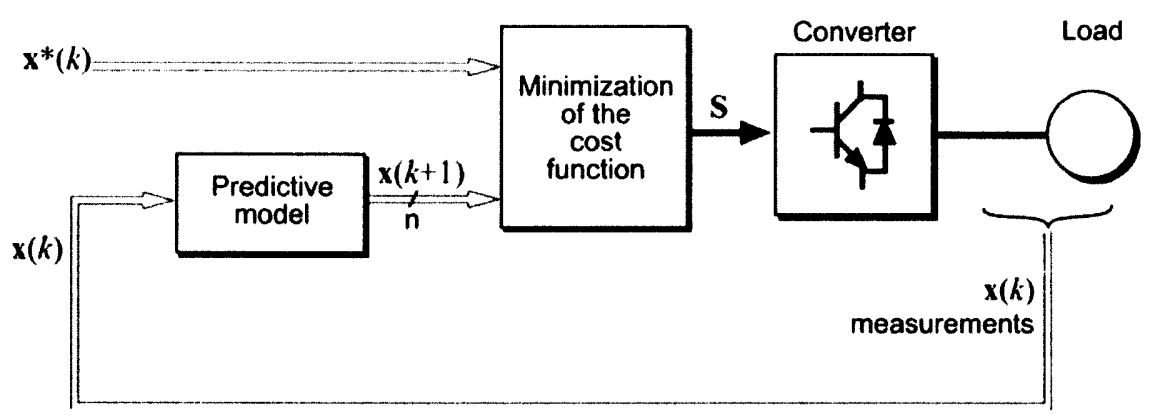

Figure 2-6: A general control diagram of FS-MPC for power converters [112], [113].

system. Based on the state-space model, FS-MPC method is able to predict all the possible future states based on a set of control actions, where the receding horizon approach is adopted for prediction. After that, a sequence of optimal control actions are determined via an optimization algorithm. These control actions have the capability to minimize the selected objectives for the whole control horizon. However, only the first action of the sequence of optimal control actions will be applied to the target system for execution. After the execution, the state of the target system will be updated by the new measurements. The whole prediction and optimization process will be repeated over a shifted prediction horizon.

In [112], the authors have pointed out that FS-MPC has a lot of advantageous features such as no modulator requirement, varying switch frequency, online optimization, low complexity and easy to incorporate constraints. Furthermore, the system model development, influence of horizon length, application examples and so on are discussed in depth. In [113], the comparison between the FS-MPC method and conventional linear methods has been conducted based on a traditional three-phase voltage source inverter. The experimental results demonstrate the aforementioned advantages of FS-MPC. Besides the advantages mentioned in [112], the FS-MPC decouples the multiple variables of the target system. Furthermore, the FS-MPC can be directly implemented on digital processors. However, the conventional linear method requires one more step of discretization if digital implementation is needed.

MPC has been gaining popularity in various applications due to its characteristics as mentioned before. It successfully regulates various kinds of power electronics including converters [114]-[119], inverters [120]-[124], and rectifiers [125]-[129].

In [114], [115], a control method combining the MPC method with a sliding mode observer has been proposed to regulate the boost converter. The sliding mode observer 
based on an enlarged state-space model is adopted to conduct implicit estimation of the immeasurable variations in source voltage and the load resistance. The proposed method can avoid the voltage overshoot when a desired voltage change happens, and reduce the current spike. In [116], the authors have proposed an enumeration-based MPC method for boost converter. The proposed method is able to realize the direct voltage regulation without underlying current control loop. To achieve the estimation of the load, which is uncertain in practical applications, a discrete-time Kalman filter is adopted. Moreover, the cost function is minimized by using an enumeration technique, which makes the implementation of MPC simpler and more practical. The proposed enumeration-based MPC method shows the features of voltage overshoot suppression and fast response to the step changes in reference and load.

The MPC method is also applied to regulate the multi-level cascaded converter for modularity and scalability in [117]. The authors have developed a modulated MPC method which improves the performance of the conventional MPC method. The proposed method inherits the ability to obtain a multi-objective control. In addition, it involves a suitable modulation scheme, which contributes to a constant switching frequency. However, the switching frequency of the conventional MPC method is varying. Therefore, the proposed modulated MPC method has a better performance in terms of current waveform total harmonic distortion over the conventional MPC method.

In [118], a control method based on the MPC strategy has been developed for an ACDC converter, which is called model predictive direct power control (MPDPC). In the MPDPC, the active power and reactive power are simultaneously controlled by considering these two parameters in the cost function. However, this kind of cost function leads to the mutual interference between the active power and reactive power. The authors have proposed a reorganized cost function to reduce or even eliminate the previous mentioned mutual interference. As a result, the dynamic performance of the AC-DC converter is improved when the active power or reactive power varies.

In [119], the MPC method is applied to an inverter with output $L C$ filter for generating sinusoidal voltages with low harmonic distortion. A current observer is adopted to conduct the estimation of the load current, which improves the system stability and avoids the additional current measurements. A fast dynamic response of the voltage regulation is achieved because the output voltage is directly controlled without using a cascaded control structure. 
An optimized MPC method has been developed to control a three-phase cascade Hbridge inverter in [120], and the proposed optimization aims to reducing the complexity of computation for MPC method. Since the three-phase cascade H-bridge inverter has a multi-level topology, it will incorporate a lot of voltage vectors resulting in the fairly high computation requirement. The authors have proposed a voltage vector selection method to determine the proper vector rather all of them so that the requirement of calculation is reduced. In addition, the voltage vector selected during a sampling interval is assumed to be similar to the one selected during the previous interval. Hence, the last selected vector is utilized to replace the voltage vectors which are nearest to the last selected vector. The number of calculation is further decreased. As a result, the complexity of computation is significantly reduced, and it makes the MPC method is practical for hardware implementation.

Similarly, the computation-reducing model is applied to achieve the calculation simplification of the MPC method in [121]. The proposed method is targeted to regulate the five-leg inverter as the driver for two motors. An adjacent vector scheme is proposed and a look-up table is built to store the feasible states of all power switches. These lead to the decrement of the number of predictions and the number of cost-function evaluations. Thus, the proposed method is able to achieve fast transient performance and individual regulation of the two motors.

In [122], a MPC-based control method has been proposed to regulate grid-tie fourlevel diode-clamped inverters. In the cost function, several parameters are considered simultaneously including the net DC-link voltage, reactive power generation to meet the grid operator request, DC-link capacitor voltages balancing, and switching frequency minimization. In other words, the proposed method takes fully advantages of the multi-objective control feature of the MPC method.

In [123], the MPC method is adopted to control the active front-end (AFE) rectifier. Since the active power and DC voltage are coupled in the AFE rectifier, it is difficult to regulate these two parameters simultaneously. A strategy to determine compatible and suitable references of active power and DC voltage has been developed. Moreover, the source current limits are incorporated into the MPC method. Compared to the PI control method, the proposed method has a better dynamic performance and avoids the integral windup problem.

An improved MPDPC has been adopted to control the PWM rectifier in [124]. Although the conventional MPDPC can achieve the steady-state operation and fast 
dynamic response, it requires high computational speed to maintain this good performance. The proposed improved MPDPC conducts the optimal duty cycle control, which splits the control period into the time slot for non-zero voltage vector and the time slot for zero voltage vector. In other words, the proposed improved MPDPC is able to process two kinds of voltage vectors in one control period. Therefore, the steadystate performance is better than that regulated by the conventional MPDPC in the same condition of computational speed. In [125], the MPDPC is modified with a vector preselection technique. During every sampling period, the proposed technique decides four vectors, and then the MPDPC method stops the switching operation of one leg with the largest input current using these four vectors. Hence, the proposed vector preselection technique can improve the performance of the MPDPC by effectively reducing the switching loss.

The MPDPC has also been applied to control the PWM rectifier under conditions of balanced and unbalanced grid voltages in [126]. The authors have presented a novel concept of the instantaneous reactive power in cost function, which results in the achievement of sinusoidal grid currents and elimination of the second grid-frequency oscillation. Another contribution is that the proposed MPDPC also takes the reactive power into account.

In [127], the enumeration-based MPC method has been developed for the cascade Hbridge multi-level rectifier. Similar to the method in [116], the proposed enumeration technique and the incorporation of constraints on the switching transitions reduce the complexity of the computation. Meanwhile, the proposed enumeration-based MPC method has the capability to regulate the cascade $\mathrm{H}$-bridge multi-level rectifier well during steady-state and dynamic operations.

Besides the practical applications of MPC in the previous research works, some theoretical studies have been conducted to analyze the features of MPC in [128]-[133]. As theoretical analysis in [128]-[130], MPC has the strong ability to deal with constraints. In this thesis, this advantage of MPC is fully utilized. Considering the practical situation and protective requirement, not only the input constraints but also the output constraints are incorporated. Therefore, compared to other control methods without the capability to handle constraints, the MPC method will achieve a more superior performance by regulating the target system under the pre-set operational limits. In addition, the employment of the MPC method usually requires a substantial amount of computational efforts. The offline MPC computation is another possible and 
promising solution, and the corresponding research works have been conducted in [131]-[133]. For example, the researchers have proved that the computation can be reduced by implementing the MPC control law as a lookup table [131], and the lookup table is based on partitioning the control law into different regions. Moreover, similar methods have also been developed in [132], [133], which determine the tradeoff between the computation requirement and the size of portioned regions. These research works demonstrate that the offline MPC computation can relieve the computational requirement of the MPC method. 


\section{CHAPTER 3 MODEL PREDICTIVE VOLTAGE CONTROL FOR SI-SIMO DC-DC CONVERTER}

In this chapter, a MPVC method is developed and applied to the SI-SIMO DC-DC converter to reduce the cross regulation, which is a critical issue for SI-SIMO DC-DC converter.

\subsection{State-SPaCE Models AND CONSTRaints ANAlysis}

\subsubsection{SI-SIMO DC-DC Buck Converter}

The SI-SIMO DC-DC buck converter shown in Figure 3-1 is used for the study to explain the proposed MPVC method. $V_{d c}$ denotes the input DC voltage source. $V$ denotes the voltage between the cathode and the ground of the diode $D$, and $i_{L}$ denotes the current flowing through the inductor $L$. For the $i$ th load $R_{i}, i_{i}$ and $v_{i}$ are the corresponding current and voltage respectively. $S_{i}$ is the switch to select the $i$ th load $R_{i}$. Notice that $i$ is an integer from 1 to $n$, where $n$ is the number of loads. $S$ is the switch between DC source $V_{d c}$ and inductor $L$ with an equivalent series resistance (ESR) $R_{L}$. For further analysis and derivation, the two states " $O N$ " and " $O F F$ " of the switch $S$ can be defined as 1 and 0 respectively.

Based on the discussion, in order to achieve the MPVC method, specific state-space predictive model is developed.

\subsubsection{State-space Model for MPVC Method}

The time multiplexing is adopted for the MPVC method so that every time slot only one load $R_{i}$ is supplied by connecting to the inductor. Assume that $S_{i}$ is closed to connect the $i$ th load $R_{i}$ and the inductor $L$, then it forms a single-output buck converter. The characteristic differential equations of inductor $L$ and capacitor $C_{i}$ of the $i$ th load $R_{i}$ are

$$
\begin{gathered}
C_{i} \frac{d v_{i}}{d t}=i_{L}-i_{i} \\
L \frac{d i_{L}}{d t}=V-v_{i}-i_{L} R_{L}
\end{gathered}
$$

The relationship of $i_{i}$ and $v_{i}$, and the relationship of $V$ and $V_{d c}$ are expressed as

$$
\begin{aligned}
& i_{i}=\frac{v_{i}}{R_{i}} \\
& V=S V_{d c}
\end{aligned}
$$




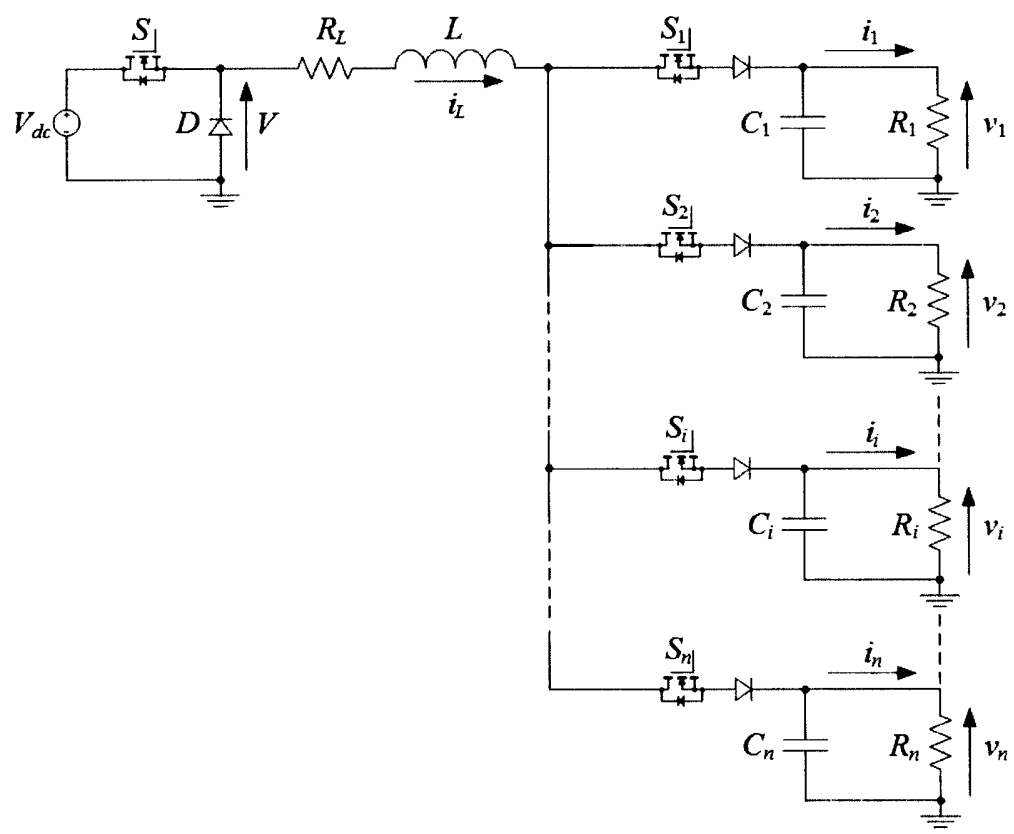

Figure 3-1: Architecture of SI-SIMO DC-DC buck converter.

By substituting (3.3) into (3.1) and substituting (3.4) into (3.2), it can be obtained as follows:

$$
\begin{gathered}
C_{i} \frac{d v_{i}}{d t}=i_{L}-\frac{v_{i}}{R_{i}} \\
L \frac{d i_{L}}{d t}=S V_{d c}-v_{i}-i_{L} R_{L}
\end{gathered}
$$

Moreover, the MPVC method is operated in discrete time with time step $T_{S}$, the continuous equations (3.5) and (3.6) must be converted to discrete format. For (3.5), the discretization format when $t$ is equal to $k$ is expressed as

$$
v_{i}(k+1)-v_{i}(k)=\frac{T_{S}}{C_{i}}\left(i_{L}(k)-\frac{v_{i}(k)}{R_{i}}\right)
$$

Thus, when $t$ is equal to $k$-1, the discrete format of (3.5) can be expressed as

$$
v_{i}(k)-v_{i}(k-1)=\frac{T_{S}}{C_{i}}\left(i_{L}(k-1)-\frac{v_{i}(k-1)}{R_{i}}\right)
$$

The left side of (3.8) is defined as $\Delta v_{i}(k)$. Similarly, $\Delta i_{L}(k)$ and $\Delta S(k)$ are defined. Using (3.7) to subtract (3.8), it can be obtained as

$$
\Delta v_{i}(k+1)=\Delta v_{i}(k)+\frac{T_{s}}{C_{i}} \Delta i_{L}(k)-\frac{T_{s}}{C_{i} R_{i}} \Delta v_{i}(k)
$$

Similarly, based on (3.6), it can be obtained that 


$$
\Delta i_{L}(k+1)=\left(1-\frac{R_{L} T_{S}}{L}\right) \Delta i_{L}(k)-\frac{T_{S}}{L} \Delta v_{i}(k)+\frac{T_{s} v_{d c}}{L} \Delta S(k)
$$

Moreover, based on the definition of $\Delta v_{i}(k+1)$, it can be obtained as

$$
v_{i}(k+1)=v_{i}(k)+\Delta v_{i}(k+1)
$$

By substituting (3.9) into (3.11), a expression of $v_{i}(k+1)$ is expressed as follows:

$$
v_{i}(k+1)=\frac{T_{s}}{C_{i}} \Delta i_{L}(k)+\left(1-\frac{T_{S}}{R_{i} C_{i}}\right) \Delta v_{i}(k)+v_{i}(k)
$$

With (3.9), (3.10) and (3.12), the augmented discrete-time state-space model for the $i$ th load is obtained as

$$
\begin{aligned}
& x_{i}(k+1)=\bar{A}_{i} x_{i}(k)+\bar{B}_{i} \Delta S(k) \\
& y_{i}(k)=\bar{C}_{i} x_{i}(k)
\end{aligned}
$$

$x_{i}(k)=\left[\Delta i_{L}(k) \Delta v_{i}(k) y_{i}(k)\right]^{T}$ is the state vector of the buck converter, and the output voltage of the buck converter is $y_{i}(k)=v_{i}(k)$. The coefficient matrices are

$$
\begin{gathered}
\bar{A}_{i}=\left[\begin{array}{ccc}
1-R_{L} T_{s} / L & -T_{s} / L & 0 \\
T_{s} / C_{i} & 1-T_{s} / R_{i} C_{i} & 0 \\
T_{s} / C_{i} & 1-T_{s} / R_{i} C_{i} & 1
\end{array}\right] \\
\bar{B}_{i}=\left[\begin{array}{lll}
T_{S} V_{d c} / L & 0 & 0
\end{array}\right]^{T} \\
\bar{C}_{i}=\left[\begin{array}{lll}
0 & 0 & 1
\end{array}\right]
\end{gathered}
$$

According to the MPC theory, predict horizon, control horizon and Lagrange multiplier are denoted as $N_{P}, N_{C}$ and $\lambda$ respectively. Moreover, the control horizon $N_{C}$ is the length of control action combination. The cost function $J_{i}$ for the $i$ th load $R_{i}$, which is used to determine the optimal control actions, is defined as follows [112]:

$$
J_{i}=\sum_{j=1}^{N_{P}}\left(y_{i}(k+j)-v_{R e f, i}(k+j)\right)^{2}+\lambda \sum_{j=1}^{N_{C}}(\Delta S(k+j-1))^{2}
$$

where $v_{R e f, i}$ is the $i$ th reference voltage for $v_{i}$. Considering that the state-space model is derived from (3.1), (3.2), (3.3) and (3.4) which are all true in DCM and CCM, the statespace model is feasible for DCM and CCM.

Moreover, the time-multiplexing method is adopted for the load selection. In order to perform the time-multiplexing method, a priority calculation algorithm is proposed as follows:

$$
p_{i}(k)>p_{j}(k), \text { if }\left(v_{i}(k)-v_{R e f, i}(k)\right) \leq\left(v_{j}(k)-v_{R e f, j}(k)\right)
$$


where $p_{i}$ and $p_{j}$ are the priorities of the corresponding $i$ th load and $j$ th load respectively. Moreover, $p_{\max }$ is defined as the maximum value among all priorities, and it will help to determine the load with highest priority.

Consequently, the selection control signal $S_{i}$ can be determined based on the corresponding priority $p_{i}$ as

$$
\left\{\begin{array}{l}
S_{i}(k)=1, \text { if } p_{i}(k)=p_{\max } \\
S_{i}(k)=0, \text { if } p_{i}(k)<p_{\text {max }}
\end{array}\right.
$$

Based on the augmented discrete-time state-space model in (3-13) and (3-16), the state-space model for the SI-SIMO DC-DC buck converter can be obtained as follows:

$$
\begin{aligned}
& x(k+1)=A X(k)+B \Delta S(k) \\
& y(k)=C X(k)
\end{aligned}
$$

$X(k)=\left[\Delta i_{L}(k) \Delta v(k) y(k)\right]^{T}$ is the state vector of the SI-SIMO DC-DC buck converter, and the output voltage is $Y(k)=v(k)$. The coefficient matrices are

$$
A=\sum_{i=1}^{n} S_{i} \bar{A}_{i} ; B=\sum_{i=1}^{n} S_{i} \bar{B}_{i} ; C=\sum_{i=1}^{n} S_{i} \bar{C}_{i}
$$

Similarly, the cost function $J$ of the SI-SIMO DC-DC buck converter is expressed as follows:

$$
J=\underbrace{\sum^{n} S_{i}\left(\sum^{N_{P}}\left(y_{i}(k+j)-v_{\text {Refi }}(k+j)\right)^{2}\right)}_{\mathbf{I}}+\underbrace{\lambda \sum_{C}^{N_{C}}(\Delta S(k+j-1))^{2}}_{\mathbf{I I}}
$$

The control actions $S$ which are able to minimize the cost function $J$ will be applied to the SI-SIMO DC-DC buck converter. It can be seen that $J$ consists of two terms I and II. Term I calculates the quadratic sum of output voltage errors, and term II penalizes the variation of the $N_{C}$ consecutive control signals. Therefore, the Lagrange multiplier $\lambda$ determines the trade-off between the accuracy of the control result and the implementation cost of the corresponding control signals.

\subsubsection{Constraints for MPVC Method}

One advantage of the MPC method is that the constraints of the control and output signals can be set according to practical situation. For the MPVC method, the constraints of $S(k)$ are defined to obtain the proper control signal based on [128]. The control signal constraints are

$$
\begin{gathered}
\Delta S(k) \in\{-1,0,1\} \\
S(k) \in\{0,1\}
\end{gathered}
$$


Moreover, in practical situation, the inductor has the current rating $I_{c r}$. If the current through the inductor $i_{L}$ exceeds $I_{c r}$, the effective inductance of the inductor drops significantly. Therefore, it is imperative to set an upper bound for $i_{L}$. Another consideration is relieving or even avoiding the overshoot. When the reference changes, there is an overshoot of the output before it becomes stable if no constraint is pre-set. Additionally, if the converter works in DCM, the inductor will endure a high peak current, especially in heavy load condition. Nevertheless, the CCM operation relieves the peak current through the inductor as discussed in [66], [67]. Hence, a lower bound is also necessary to guarantee the SIMO DC-DC buck converter to work in CCM. As a result, the constraint for $i_{L}$ is set as follows:

$$
\alpha I_{c r} \leq i_{L} \leq \beta I_{c r}
$$

where $\alpha$ and $\beta$ are the adjustable coefficients, and they fulfill the relationship $0<\alpha<$ $\beta<1$. By properly setting the values of $\alpha$ and $\beta$, the current of inductor $i_{L}$ is regulated in a suitable and proper varying range so that it contributes to small ripple of the output voltage.

\subsection{MPVC METHOD}

\subsubsection{MPVC Controller}

The MPVC controller is shown in Figure 3-2. Generally, the quadratic programming algorithm is adopted to determine the optimal solution of the cost function. However, the quadratic programming algorithm is hard to be implemented in the DSP controller. Therefore, an enumerated algorithm is utilized for the practical implementation, which has been applied into MPC method for a single-input single-output boost converter [116]. The data $\left(v, i, i_{L}\right)$ and matrices $(A, B, C)$ for the MPVC controller will be updated and sent to the prediction block. Consequently, all possible future states $\left(y^{1}, y^{2}, \ldots, y^{m}\right)$ of the SI-SIMO converter can be predicted. The number of the possible predicted states $m$ is equal to $2^{N c}$, because the number of all permutations and combinations of $N_{C}$ steps of control signal $S$ is $2^{N c}$, as shown in Figure 3-2. Then, the selected reference voltage $v_{\text {Ref }}$ and all possible future states $\left(y^{1}, y^{2}, \ldots, y^{m}\right)$ are sent to the minimization block, and then substituted into the cost function $J$ to calculate a series results $\left(J^{1}, J^{2}, \ldots, J^{m}\right)$. If $J^{k}$ is equal to the minimum value $J_{\min }$ of $\left(J^{1}, J^{2}, \ldots, J^{m}\right)$, the corresponding $k$ th control action combination is the optimal combination. Therefore, the first control action of the $k$ th 


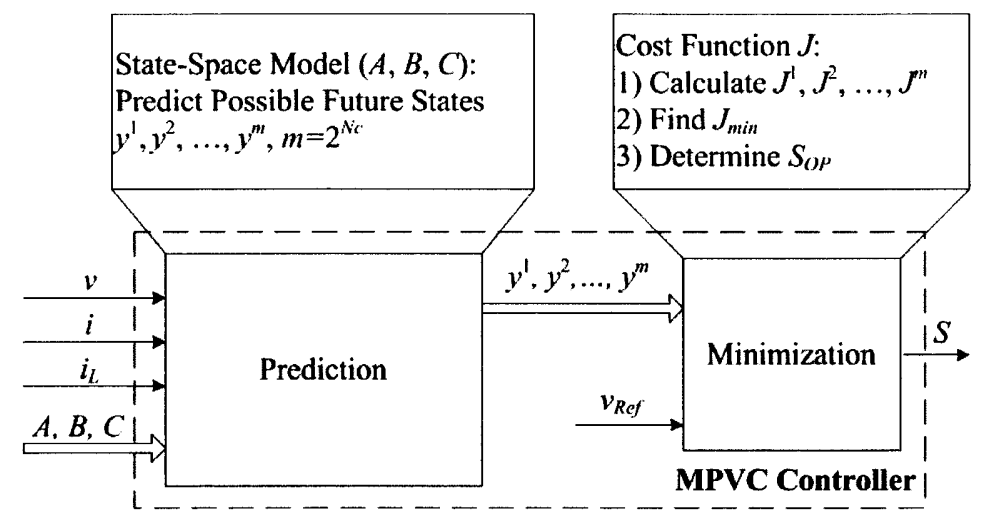

Figure 3-2: Block diagram of MPVC controller.

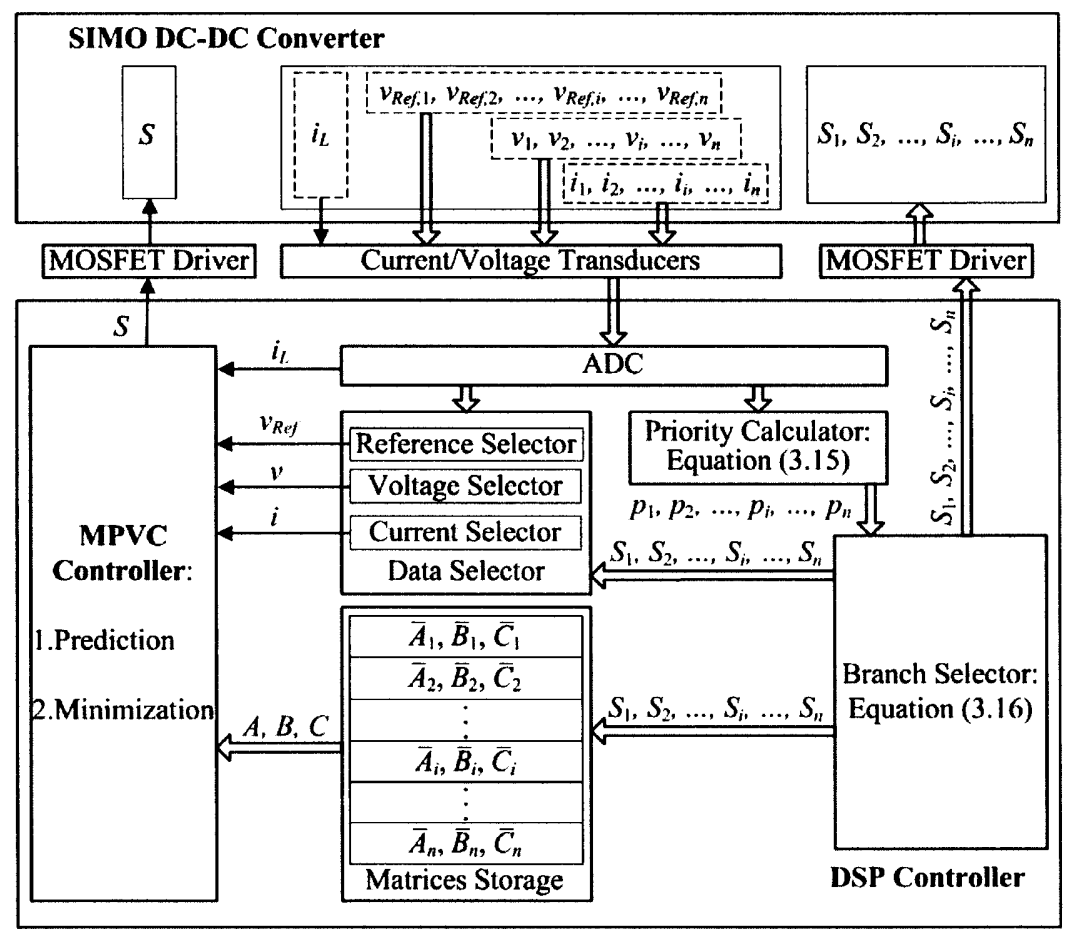

Figure 3-3: Architecture of SI-SIMO DC-DC buck converter with MPVC controller.

control action combination is regarded as the optimal control action $S_{O P}$. This $S_{O P}$ will be applied to the SI-SIMO converter as the control action $S$ for next control cycle.

\subsubsection{Architecture of SI-SIMO DC-DC Converter with MPVC Controller}

With MPVC controller, it is possible to achieve MPVC method for a general SISIMO DC-DC converter. Actually, the most important step is to deduce the discretetime state-space model defining the characteristics of the corresponding single-output DC-DC converter, which is the basic unit of the SI-SIMO DC-DC converter. This statespace model will help to build the MPVC controller. Figure 3-3 presents a universal 


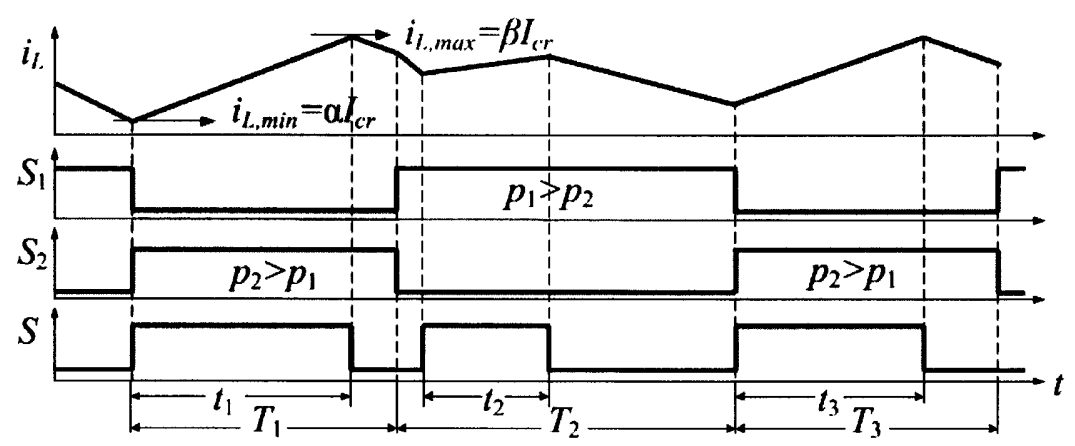

Figure 3-4: MPVC control scheme for single-inductor single-input dual-output (SI-SIDO) buck converter.

architecture of the SI-SIMO DC-DC converter with MPVC controller, which is implemented in a DSP controller. Firstly, the real-time data $\left(v_{i}, i_{i}\right)$ of all loads, where $i$ is from 1 to $n$, and inductor current $i_{L}$ are sent to the DSP controller through the current/voltage transducers and ADC block. As discussed before, the time-multiplexing method is applied. Therefore, the priority algorithm is imperative to decide which load to be connected to the inductor $L$ during every time slot. With the priority $\left(p_{1}, p_{2}, \ldots\right.$, $\left.p_{i}, \ldots, p_{n}\right)$, the branch selector will generate the proper selection signals $\left(S_{1}, S_{2}, \ldots, S_{i}, \ldots\right.$, $S_{n}$ ). Consequently, the data of the prior load and matrices for the MPVC controller will be determined and sent to the MPVC controller to generate the optimal control action $S$. Moreover, the branch selector will also connect the prior load to the inductor and disconnect the others at the same time with the selection signals $\left(S_{1}, S_{2}, \ldots, S_{i}, \ldots, S_{n}\right)$. All control signals are applied to the SI-SIMO DC-DC converter through the drivers.

\subsubsection{Control Scheme of MPVC Method}

In order to explain the control scheme of the MPVC method, a single-inductor singleinput dual-output (SI-SIDO) DC-DC buck converter is proposed for the study by setting the number $n$ of the load as 2 in Figure 3-1, which is the simplest SI-SIMO DC-DC buck converter. Figure 3-4 shows the control scheme. In time slots $T_{1}$ and $T_{3}$, the states of $S_{2}$ are " $O N$ ", which means that $R_{2}$ has higher priority $p_{2}$ than $p_{1}$ of $R_{1}$, so that $R_{2}$ is connected to the inductor $L$. On the contrary, $p_{1}$ is greater than $p_{2}$ in time slot $T_{2}$. The corresponding duty cycles are $t_{1} / T_{1}, t_{2} / T_{2}$ and $t_{3} / T_{3}$ respectively. The values of $T_{1}, T_{2}$ and $T_{3}$ are determined by the priority algorithm, and the values of $t_{1} / T_{1}, t_{2} / T_{2}$ and $t_{3} / T_{3}$ are determined dynamically and immediately by the MPVC controller based on real-time data $\left(v, i, v_{R e f}, i_{L}\right)$ conditions. Therefore, the proposed MPVC method is able to regulate the SI-SIMO DC-DC converter and suppress the cross regulation successfully with the 
Table 3-1 Simulation parameters of the SIDO DC-DC buck converter

\begin{tabular}{c|c|c|c|c|c|c|c|c}
\hline \hline Symbol & $V_{d c}$ & $v_{1}$ & $v_{2}$ & $R_{i}$ & $T_{S}$ & $R_{L}$ & $L$ & $C_{i}$ \\
\hline Value & $20 \mathrm{~V}$ & $12 \mathrm{~V}$ & $8-12 \mathrm{~V}$ & $10 \Omega$ & $5 \mu \mathrm{s}$ & $0.046 \Omega$ & $100 \mu \mathrm{H}$ & $100 \mu \mathrm{F}$ \\
\hline
\end{tabular}

rapid and dynamic response function. Moreover, the inductor current $i_{L}$ is in a pseudo steady state resulting from the dynamic duty cycles of $S$. It can also be seen that the inductor current $i_{L}$ is between $\alpha I_{c r}$ and $\beta I_{c r}$ due to the constraints, which guarantees that the converter works in the CCM and avoids high peak current.

\subsubsection{Design Consideration for MPVC Method}

The selection of the inductor and capacitors for the proposed MPVC method is based on the equations from [134] as shown below.

$$
\begin{gathered}
L=\left(V_{d c}-v_{i}\right) \frac{v_{i}}{V_{d c} f_{s w} \Delta i_{L}} \\
C_{i}=\frac{L\left(i_{i}+0.5 \Delta i_{L}\right)^{2}}{\left(v_{i}+\Delta v_{i}\right)^{2}-v_{i}^{2}}
\end{gathered}
$$

where $f_{s w}$ is the switching frequency of signal $S, \Delta i_{L}$ is the ripple of inductor current $i_{L}$ and $\Delta v_{i}$ is the output voltage ripple.

The MPVC method is different from the conventional methods that it has a varying switching frequency $f_{s w}$ as shown in Figure 3-4. However, it is still possible to estimate the value of $f_{s w}$. As the time step $T_{s}$ is set for discrete time operation, the maximum value $f_{s w, \max }$ can be obtained as

$$
f_{s w, \max }=\frac{1}{2 T_{s}}
$$

In [135], the authors have pointed out that the output filter has to been designed based on the lowest possible switching frequency in order to provide the required attenuation for ripple under all operation conditions. However, due to the varying switching frequency, the minimum value $f_{s w, \min }$ is set as $0.2 f_{s w, \max }$ for robust consideration.

For $\Delta i_{L}$, it cannot be estimated by $0.3 i_{i}$ as estimated in the conventional methods [134], considering that it is operated in a pseudo steady state. As $i_{L}$ has the constraints in (3.20), $\Delta i_{L}$ is set as $(\beta-\alpha) I_{c r}$. Moreover, $\Delta v_{i}$ is set as $5 \% v_{i}$. Therefore, the values of inductor and capacitors can be determined with the information of $V_{d c}, v_{i}$ and $i_{i}$. The design values of the inductor and capacitors are discussed in following sections based on (3.21) and (3.22). 


\subsection{Simulation Results}

To evaluate the performance of the proposed MPVC method for the SI-SIMO DCDC converter, simulation studies are conducted in MATLAB/Simulink based on the SI-SIDO buck converter as shown in Figure 3-1. The values of $V_{d c}, v_{1}$ and $v_{2}$ are set as $20 \mathrm{~V}, 12 \mathrm{~V}$ and $8-12 \mathrm{~V}$. The load resistor is $10 \Omega$ so that $i_{1}$ is $1.2 \mathrm{~A}$ and $i_{2}$ is $0.8 \mathrm{~A}$. Moreover, the time step $T_{s}$ is set as $5 \mu$ s considering the feasibility for hardware implementation. Therefore, the minimum value of $f_{s w, \min }$ is $20 \mathrm{kHz}$ for robust consideration.. By substituting $V_{d c}=20 \mathrm{~V}, v_{1}=12 \mathrm{~V}, v_{2}=8 \mathrm{~V}, i_{1}=1.2 \mathrm{~A}, i_{2}=0.8 \mathrm{~A}, f_{s w}=20 \mathrm{kHz}$ into (3.21) and assuming $\Delta i_{L}=3 \mathrm{~A}$, the value of inductor $L$ can be calculated as $80 \mu \mathrm{H}$ for $v_{1}=12 \mathrm{~V}$ and $v_{2}=8 \mathrm{~V}$ condition. Moreover, considering the typical tolerance $(20 \%)$ of the inductance, the value of inductor is selected as $100 \mu \mathrm{H}$, and the corresponding internal resistance $R_{L}$ is $0.046 \Omega$ based on the datasheet of an inductor named as BOURNS 1140-101K-RC. Consequently, the two values of the capacitor $C_{i}$ are $49.4 \mu \mathrm{F}$ and $80.6 \mu \mathrm{F}$ based on (3.22). Similarly, considering the robustness and the typical tolerance $(20 \%)$ of the capacitance, the value of capacitor is selected as $100 \mu \mathrm{F}$. The simulation parameters of the buck converter for the MPVC method are shown in Table 3-1. The prediction horizon $N_{P}$, control horizon $N_{C}$ and Lagrange multiplier $\lambda$ are set differently and their influences are analyzed in the following simulations.

\subsubsection{Steady-state of MPVC Method}

\subsubsection{Influences of Control Horizon $N_{C}$, Lagrange Multiplier $\lambda$ and Prediction Horizon $N_{P}$}

To guide the control parameter settings of the hardware implementation, the influences of the MPVC parameters $N_{P}, N_{C}$ and $\lambda$ on the voltage ripple are studied in simulation based on a single-output buck converter. Considering the structure of (3.18), $N_{C}$ and $\lambda$ are studied simultaneously because they are relevant to the control signals in term II, and $N_{P}$ is studied alone since it determines the predicted future states in term $\mathbf{I}$.

The influences of $N_{C}$ and $\lambda$ on the voltage ripple are studied with condition $N_{P}=12$ and $V_{R e f}=5 \mathrm{~V}$. Simulation results are shown in Figure 3-5(a). It can be seen that $N_{C}$ varies from 1 to 10 and $\lambda$ varies from 0 to 0.2 . A series of simulations are conducted for different combinations of $N_{C}$ and $\lambda$. The grey curved surface shows the voltage ripples 


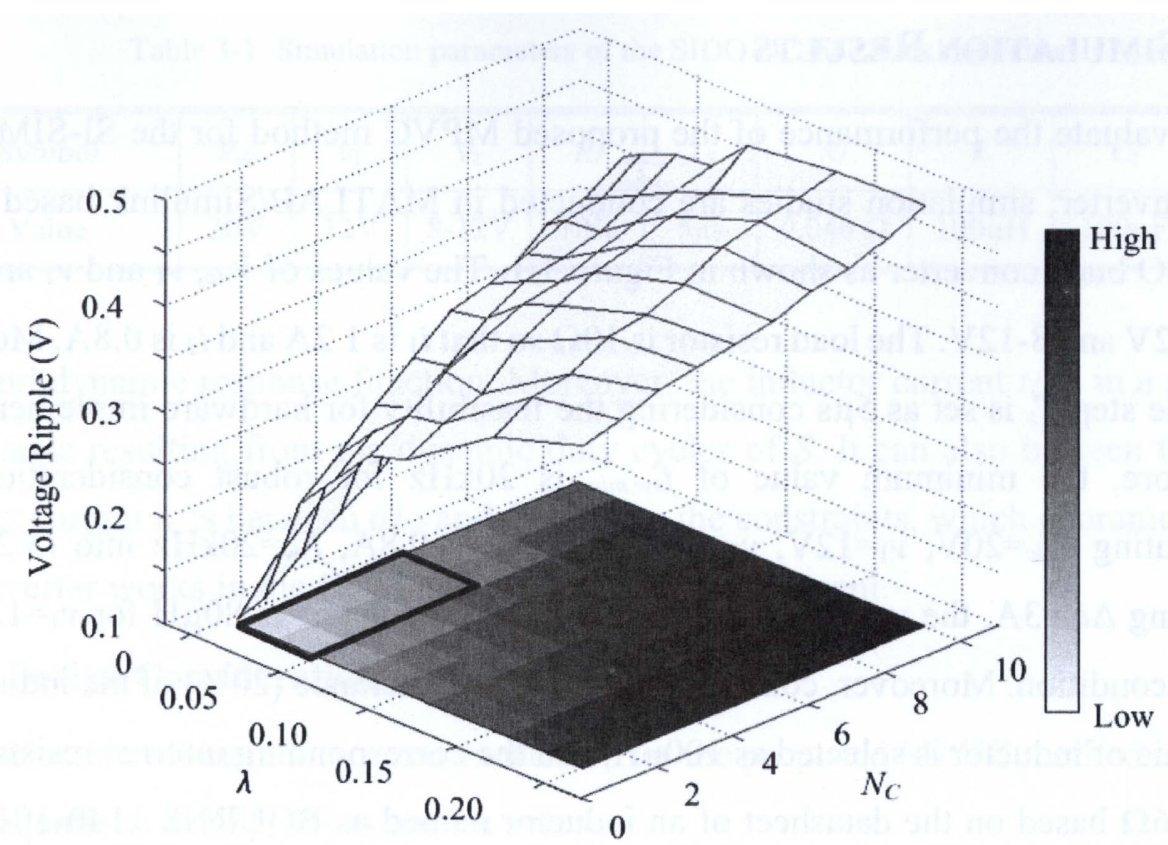

(a)

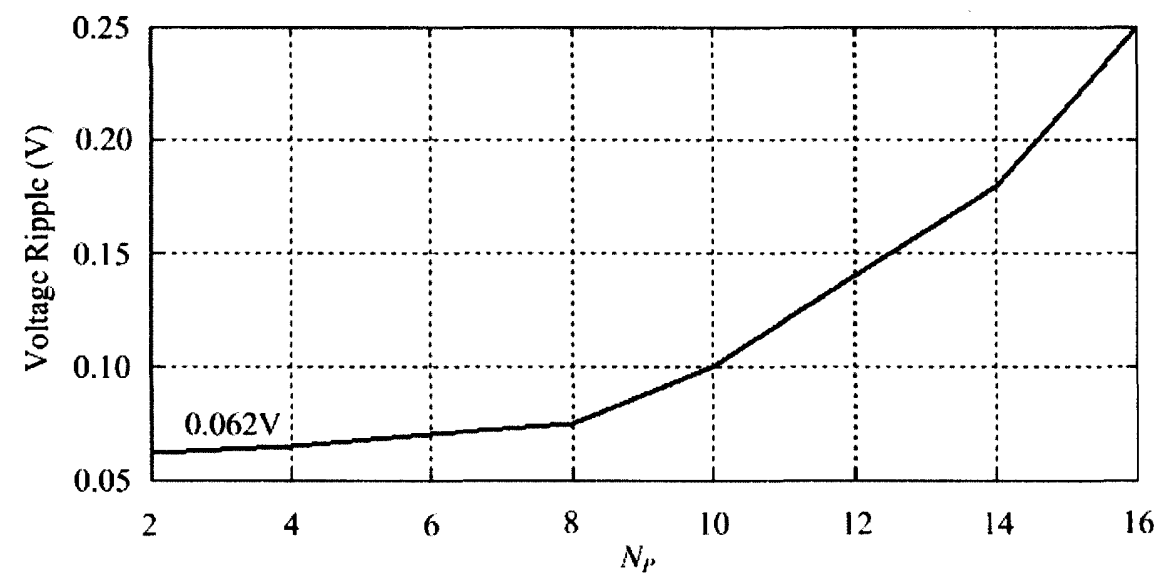

(b)

Figure 3-5: Influences of the control horizon $N_{C}$ and Lagrange multiplier $\lambda$ of MPVC method when $T_{S}=5 \mu \mathrm{s}$ and $V_{R e f}=5 \mathrm{~V}$. (a) Influence of $N_{C}$ and $\lambda$ when $N_{P}=12$. (b) Influence of $N_{P}$ when $N_{C}=1, \lambda=0.001$.

under different conditions. In order to show the influences clearly, the curved surface is projected onto the $\left(N_{C}, \lambda\right)$ plane. Therefore, it can be observed that the optimal parameter settings for the MPVC method are $1 \leq N_{C} \leq 5$ and $0<\lambda \leq 0.05$ (the emphasized rectangular region of the projection), which make the average voltage ripples fairly less than the reference voltage. Hence, for better voltage regulation, small $N_{C}$ and $\lambda$ can be selected for the MPVC method. The influence of the predict horizon $N_{P}$ on the voltage ripple has been studied with the condition $N_{C}=1, \lambda=0.001$ and $V_{R e f}=5 \mathrm{~V}$. The simulation results are shown in Figure 3-5(b). The voltage ripple is reduced when $N_{P}$ is decreased from 16 to 2 . Hence, the MPVC method requires small $N_{P}$ for better voltage regulation. 


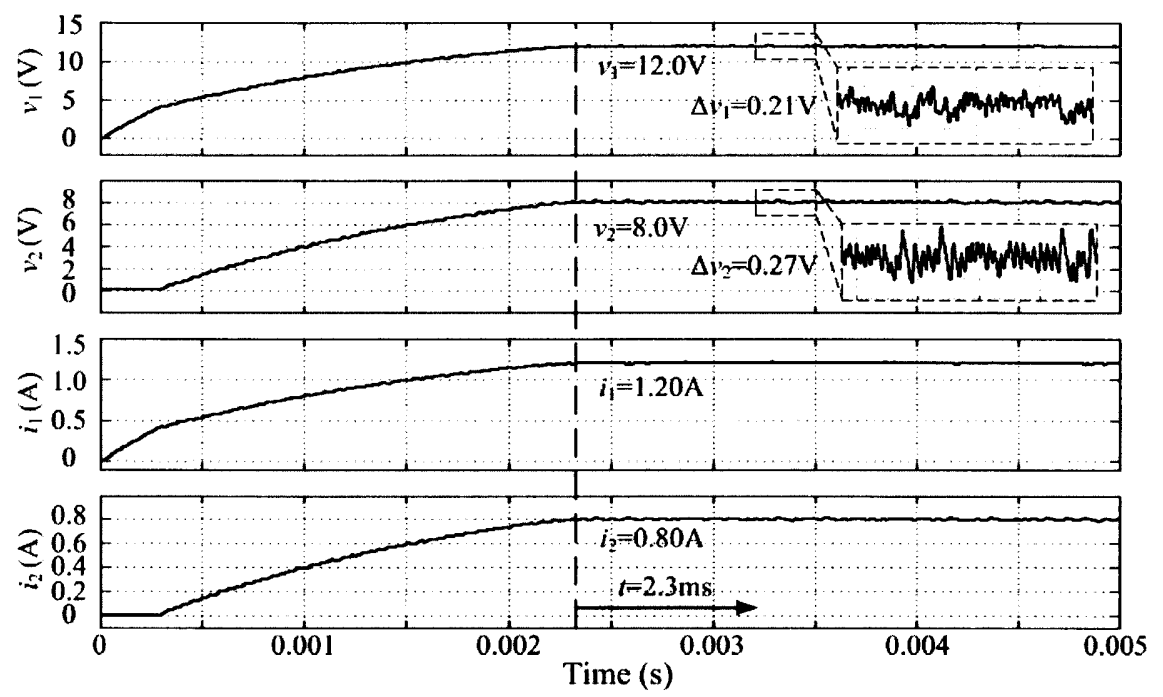

Figure 3-6: Steady-state simulation waveforms of the proposed MPVC method based on SI-SIDO buck converter as shown in Figure 3-1.

For hardware implementation, it also requires small values of $N_{P}$ and $N_{C}$ for improving dynamic performance, considering the computational complexity of the proposed method has a positive correlation with $N_{P}$ and $N_{C}$.

\subsubsection{Steady-state Operation of MPVC Method}

Based on the SI-SIDO buck converter built in Simulink, the steady-state operation of the proposed MPVC method is studied. The simulation results of the MPVC method are shown in Figure 3-6. The $N_{P}, N_{C}$ and $\lambda$ are set as 2, 1 and 0.001 based on the analysis in Figure 3-5. The reference voltages $v_{R e f, 1}$ and $v_{R e f, 2}$ are set as $12 \mathrm{~V}$ and $8 \mathrm{~V}$ respectively. It can be seen that it take $2.3 \mathrm{~ms}$ for the MPVC method to regulate the two output voltages $v_{1}$ and $v_{2}$ to track the reference voltages closely. In detail, the voltage ripples $\Delta v_{1}$ and $\Delta v_{2}$ are $0.21 \mathrm{~V}$ and $0.27 \mathrm{~V}$, which are $1.75 \%$ of $v_{R e f, 1}$ and $3.37 \%$ of $v_{R e f, 2}$ respectively. Correspondingly, the output currents $i_{1}$ and $i_{2}$ are approximate $1.20 \mathrm{~A}$ and $0.80 \mathrm{~A}$ respectively. Therefore, the cross regulation is significantly suppressed by the proposed MPVC control method based on its dynamic and fast control functions.

\subsubsection{Dynamic Performance of MPVC Method}

In this subsection, the dynamic performance of the proposed MPVC method is studied based on the SI-SIDO buck converter as shown in Figure 3-1 including the transient response to reference voltage variation and the load regulation. The simulation results and the corresponding discussions are presented below. Similarly, $N_{P}, N_{C}$ and $\lambda$ are set as 2,1 and 0.001 respectively. 


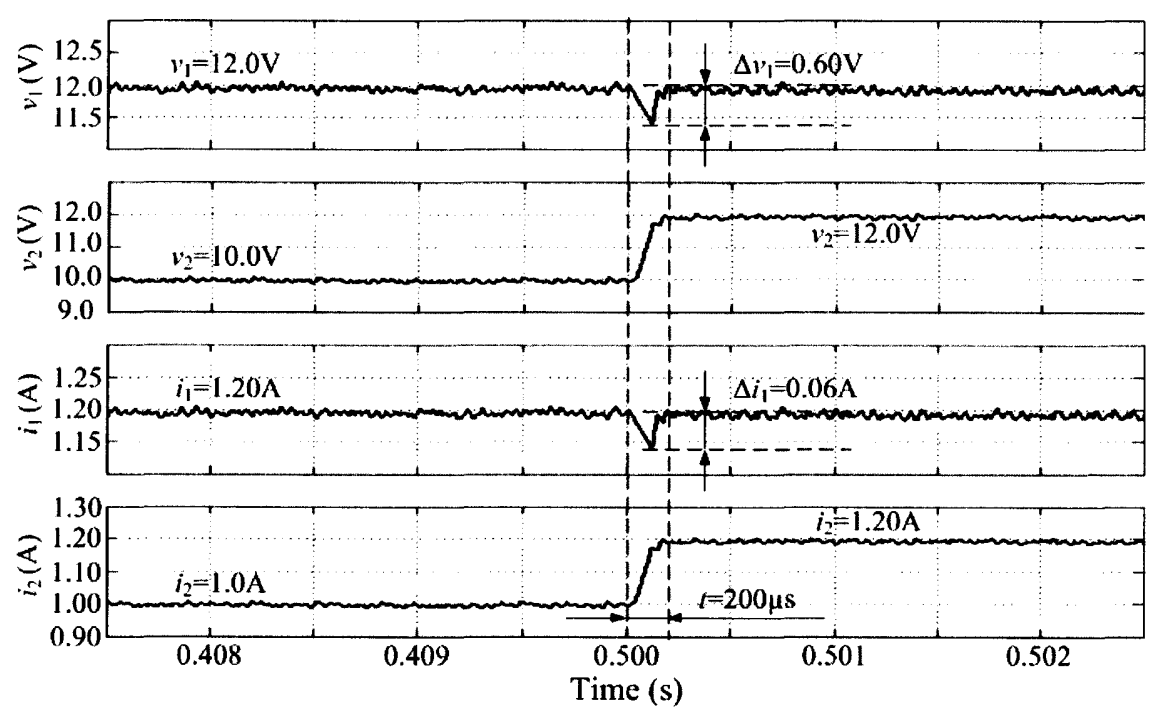

Figure 3-7: Transient response to the reference change simulation waveforms of the proposed MPVC method based on SI-SIDO buck converter as shown in Figure 3-1.

\subsubsection{Transient Response to Reference Voltage Variation}

The transient response of the MPVC method to the reference voltage change is studied, and the simulation results are shown in Figure 3-7. At the beginning, the reference voltages $v_{R e f, 1}$ and $v_{R e f, 2}$ are set as $12 \mathrm{~V}$ and $10 \mathrm{~V}$ respectively. At $t=0.5 \mathrm{~s}, v_{R e f, 2}$ is changed from $10 \mathrm{~V}$ to $12 \mathrm{~V}$. It can be observed that it takes about $200 \mu \mathrm{s}$ for the MPVC method to regulate $v_{2}$ to follow the reference change. Consequently, the output current $i_{2}$ is increased from 1.0A to $1.20 \mathrm{~A}$. Moreover, the step change in $v_{R e f, 2}$ has influence on $v_{1}$ waveform, which is a reflection of the cross regulation and priority algorithm. Specifically, a drop of $\Delta v_{1}$ equal to $0.60 \mathrm{~V}$ during the increasing process of $v_{2}$. Accordingly, the output current $i_{1}$ also has a drop of about $0.06 \mathrm{~A}$. This is because the step change in $v_{R e f, 2}$ makes $R_{2}$ to have higher priority than $R_{1}$ and the MPVC allows $v_{1}$ to freely discharge during a certain time slot. However, $v_{1}$ and $i_{1}$ recover to $12 \mathrm{~V}$ and $1.20 \mathrm{~A}$ fast after the MPVC method realizes the drop of $v_{1}$ and $i_{1}$. The transient response indicates that when there is a reference voltage variation, it takes short time for the MPVC method to respond to this variation. 

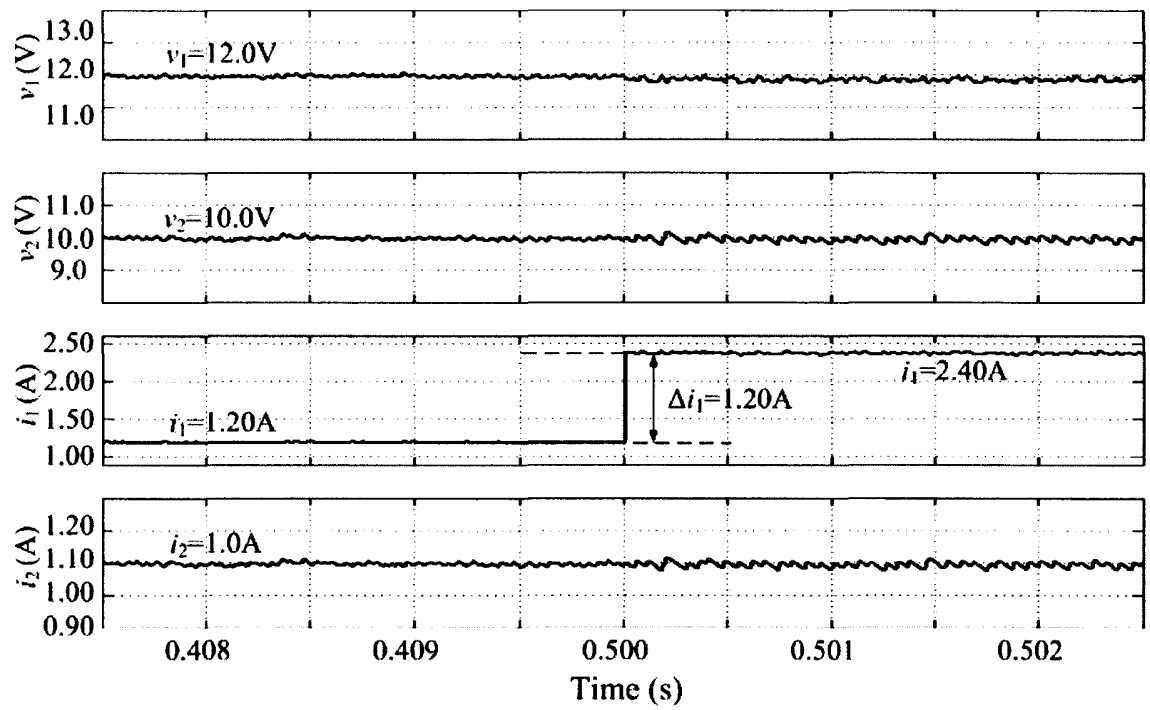

(a)
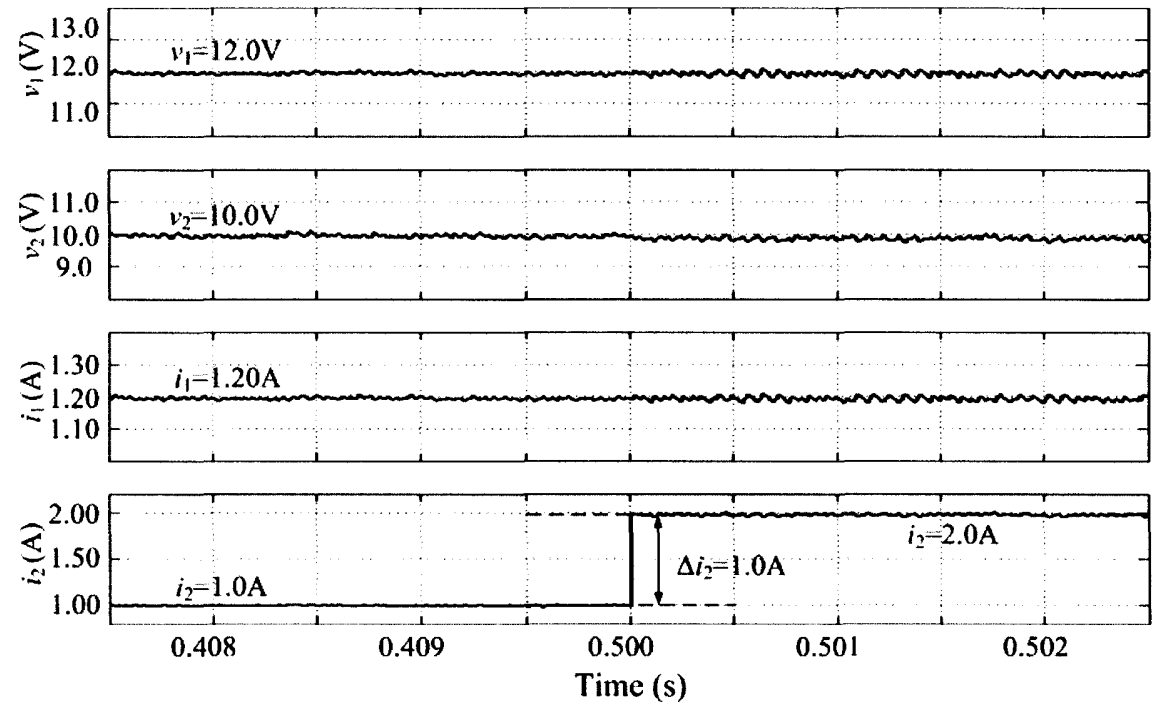

(b)

Figure 3-8: Load regulation simulation waveforms of the proposed MPVC method based on the SISIDO buck converter as shown in Figure 3-1. (a) Step change in $R_{1}$. (b) Step change in $R_{2}$.

\subsubsection{Load Regulation}

The load regulation waveforms of the proposed MPVC method for the SI-SIDO buck converter are shown in Figure 3-8. In Figure 3-8(a), there is a step change in $R_{1}$ from $10 \Omega$ to $5 \Omega$ at $t=0.5 \mathrm{~s}$. It can be seen that the output current $i_{1}$ is increased correspondingly from $1.20 \mathrm{~A}$ to $2.40 \mathrm{~A}$ with an increment of $1.20 \mathrm{~A}$, i.e., $100 \%$ increment of the load. In Figure 3-8(b), a step change in $R_{2}$ from $10 \Omega$ to $5 \Omega$ is set, and $i_{2}$ is increased from $1.0 \mathrm{~A}$ to $2.0 \mathrm{~A}$ accordingly. In Figure $3-8, v_{1}$ and $v_{2}$ are regulated to follow the reference 


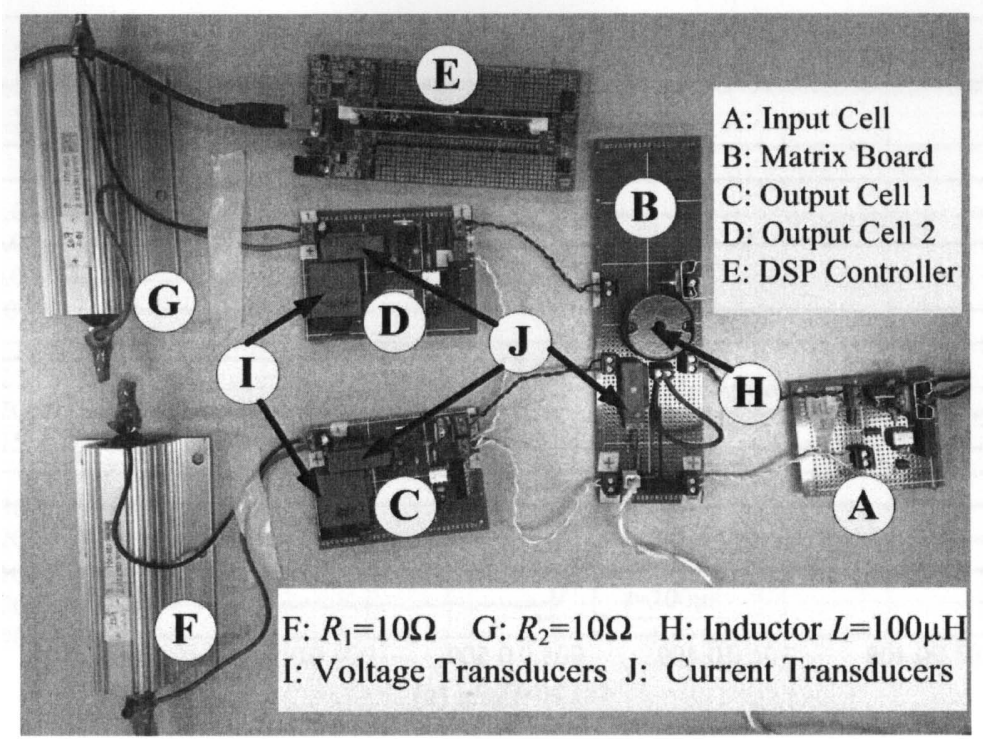

Figure 3-9: Hardware platform for verification of the proposed MPVC method.

Table 3-2 Main components adopted in hardware platform

\begin{tabular}{ll}
\hline \hline Component & Device \\
\hline MOSFET $\left(S, S_{i}\right)$ & IRF540 \\
MOSFET driver & HCPL-J314 \\
Diode $(D)$ & C4D10120D \\
Inductor $(L)$ & BOURNS 1140-101K-RC \\
Current transducer & LAH 25-NP \\
Voltage transducer & LV 25-P \\
DSP controller & TI TMS320F28335 \\
\hline \hline
\end{tabular}

voltages closely before and after the step load change in $R_{1}$ or $R_{2}$. Therefore, the proposed MPVC method is able to cope with the step load change.

\subsection{EXPERIMENTAL RESULTS}

In this section, an experimental hardware platform has been built to verify the proposed MPVC method for the SI-SIMO DC-DC converter as shown in Figure 3-9. It consists of the SI-SIDO buck converter and a DSP controller TI TMS320F28335. The main components adopted in the hardware platform are shown in Table 3-2. The saturation current $I_{c r}$ of inductor BOURNS $1140-101 \mathrm{~K}-\mathrm{RC}$ is $10.5 \mathrm{~A}$, the response time of current transducer LAH 25-NP and voltage transducer LV 25-P are 500ns and 40us 


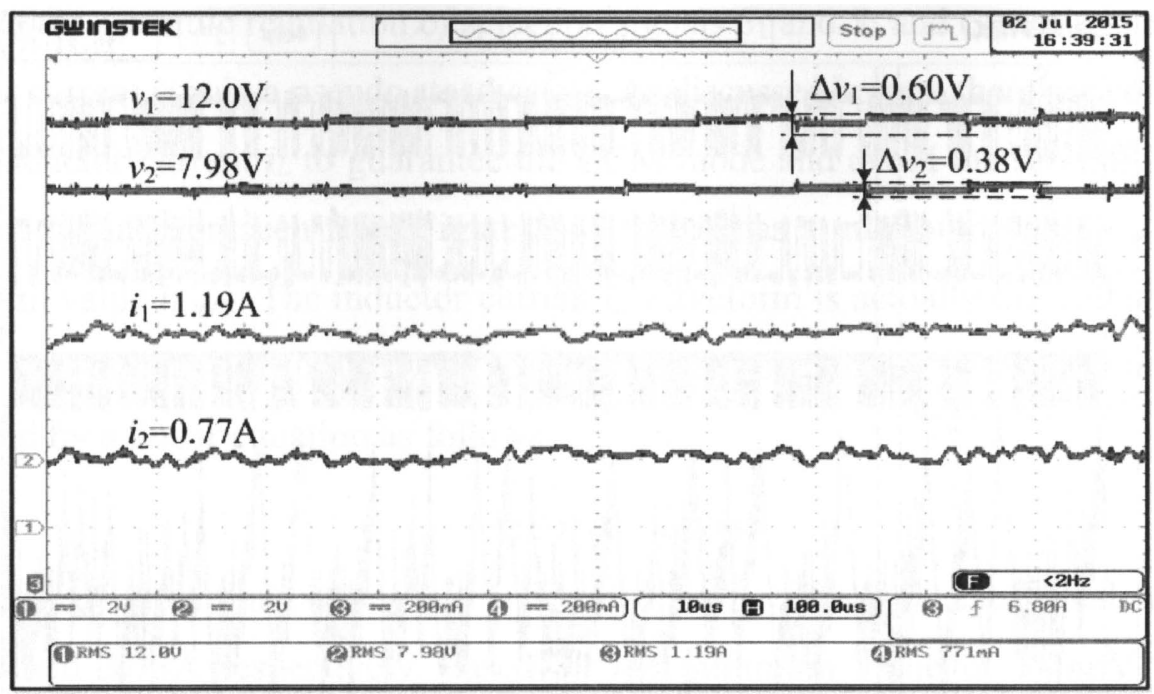

Figure 3-10: Steady-state waveforms of the proposed MPVC method. Output voltages $v_{1}, v_{2} \sim 2 \mathrm{~V} / \mathrm{div}$, Output currents $i_{1}, i_{2} \sim 200 \mathrm{~mA} / \mathrm{div}$, time $\sim 10 \mu \mathrm{s} / \mathrm{div}$.

respectively. The DSP controller TI TMS320F28335 has a maximum operating frequency of $150 \mathrm{MHz}$ and a maximum $\mathrm{ADC}$ sampling frequency of $12.5 \mathrm{MHz}$.

The MPVC control algorithm is coded in $\mathrm{C}++$ using Code Composer Studio 5.5. For the inductor current $I_{L}$, the constraint coefficients are properly set as $\alpha=1 / 25$ and $\beta=1 / 2$ in the experiment of the MPVC method, i.e., the tolerable minimum and maximum values of $i_{L}$ are $i_{L, \min }=0.42 \mathrm{~A}$ and $i_{L, \max }=5.25 \mathrm{~A}$. The experimental results for the proposed MPVC method to minimize the cross regulation and the dynamic performance of the system are discussed in the following test cases. Based on the simulation results of Figure 3-5 and Figure 3-6, the control parameters $N_{P}, N_{C}$ and $\lambda$ are set as 2, 1 and 0.001. The step time $T_{S}$ is $5 \mu \mathrm{s}$. The experimental results, including the steady-state operation, dynamic performance, efficiency and the comparison with the existing methods for the SI-SIMO DC-DC converters, are discussed in the following studies.

\subsubsection{Steady-state of MPVC Method}

Based on the hardware platform, the steady-state operation of the proposed MPVC method is studied in this subsection. The experimental waveforms of output voltages $v_{1}$ and $v_{2}$, and output currents $i_{1}$ and $i_{2}$ are shown in Figure 3-10. Moreover, selection signals $S_{1}$ and $S_{2}$, control signal $S$ and inductor current $i_{L}$ are shown in Figure 3-11. 


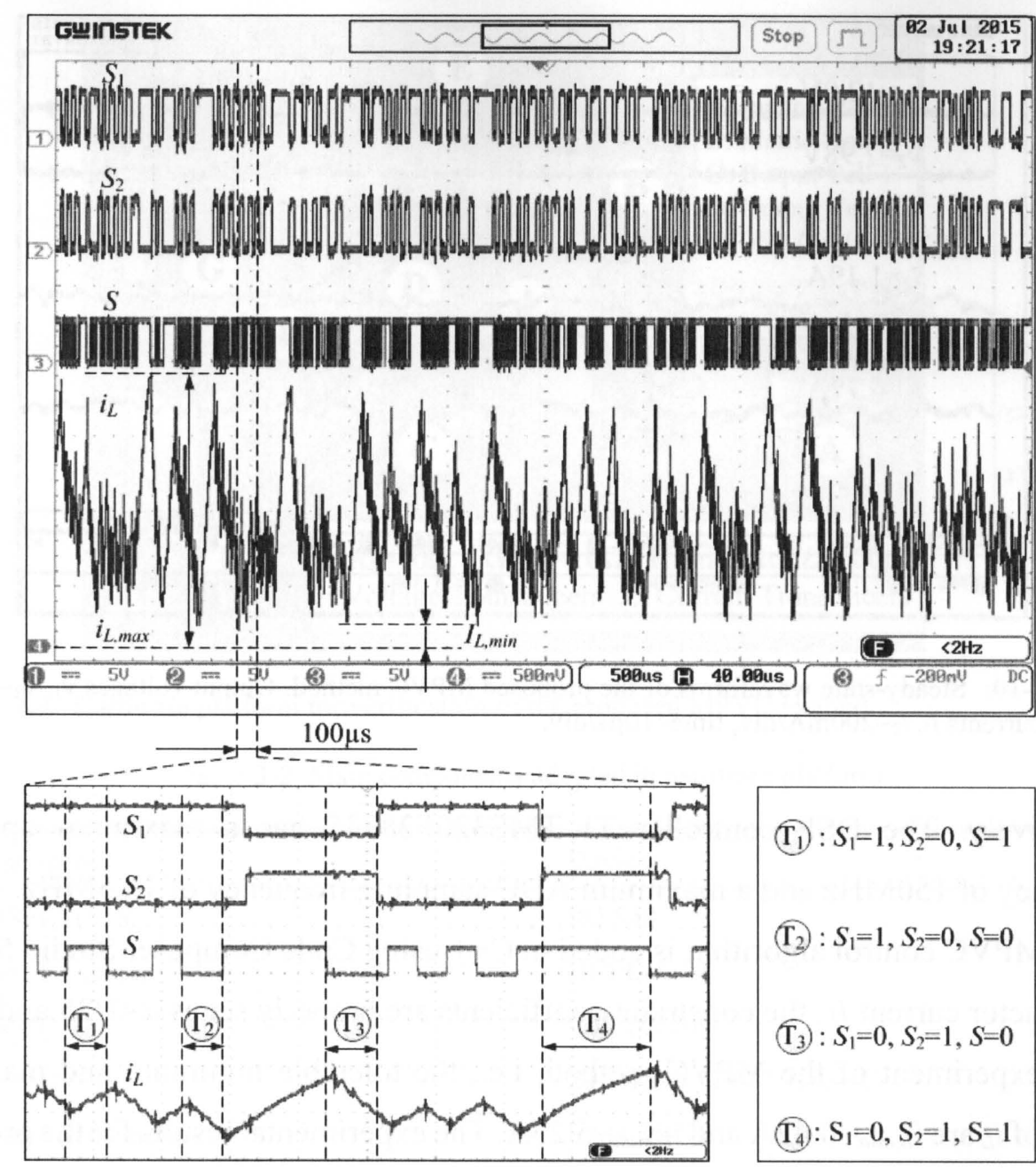

Figure 3-11: Steady-state waveforms of selection signals $S_{1}$ and $S_{2}$, control signal $S$ and inductor current $i_{L}$

In Figure 3-10, the reference voltages $v_{R e f, 1}$ and $v_{\text {Ref, } 2}$ are set as $12 \mathrm{~V}$ and $8 \mathrm{~V}$. It can be observed that the voltage ripples are $\Delta v_{1}=0.60 \mathrm{~V}$ and $\Delta v_{2}=0.38 \mathrm{~V}$, and the ripples are $5 \%$ of $v_{R e f, 1}$ and $4.75 \%$ of $v_{R e f, 2}$ respectively. Correspondingly, the output currents $i_{1}$ and $i_{2}$ are $1.19 \mathrm{~A}$ and $0.77 \mathrm{~A}$ respectively. Therefore, the proposed MPVC method is able to regulate the SI-SIDO buck converter and reduce the cross regulation.

An overview of selection signals $S_{1}$ and $S_{2}$, control signal $S$ and inductor current $i_{L}$ are shown in the top figure of Figure 3-11. It can be seen that the selection signal $S_{1}$ and $S_{2}$ are dynamically controlled according to the updated priority $p_{1}$ and $p_{2}$. Moreover, the main control signal $S$ is also dynamically adjusted by the proposed MPVC method. These three signals contribute to the dynamic response to the real-time data and suppression of cross regulation for the SI-SIDO buck converter. 
Due to the dynamic regulation of selection signals $S_{1}$ and $S_{2}$ and control signal $S$, the inductor current $i_{L}$ is in a pseudo steady state. As discussed before, there are constraints of the inductor current $i_{L}$ to guarantee the CCM mode and avoid the saturation of the inductor. It can been seen from Figure 3-11 that $i_{L}$ has a minimum value $i_{L, \min }$ and a maximum value $i_{L, \max }$. The inductor current $i_{L}$ waveform is actually the voltage signal $V_{I L}$ from the current transducer LAH 25-NP, and the relationship between $i_{L}$ and $V_{I L}$ is described by a linear equation as follows:

$$
i_{L}=2.513 V_{L L}-0.0357
$$

Therefore, a minimum value $i_{L, \min }$ and a maximum value $i_{L, \max }$ can be calculated as $0.416 \mathrm{~A}$ and $3.73 \mathrm{~A}$ respectively, based on the minimum value of $180 \mathrm{mV}$ and the maximum value of $1.5 \mathrm{~V}$ of $V_{I L}$. This inductor current $i_{L}$ follows the preset constraints well. This guarantees that the SI-SIDO buck converter works in CCM and the inductor is away from saturation.

A detailed zoom-in view in $100 \mu \mathrm{s}$ is shown in the bottom figure of Figure 3-11. There are four time slots $T_{1}, T_{2}, T_{3}$ and $T_{4}$ picked to illustrate the detail of the MPVC method. For example, in $T_{1}$ the selection signals are $S_{1}=1$ and $S_{2}=0$ so that the load $R_{1}$ is selected due to its higher priority. The control signal $S$ is 1 , therefore the inductor begins to be charged and $i_{L}$ is increased. Similarly, the waveform of $i_{L}$ follows the signals $S_{1}, S_{2}$ and $S$ in $T_{2}, T_{3}$ and $T_{4}$. Moreover, the different slopes of $i_{L}$ result from the different reference voltage settings of two loads.

In summary, the proposed MPVC method regulates the SI-SIDO buck converter in steady state successfully, and the cross regulation is significantly reduced. This capability results from the dynamic and rapid adjustment of the control signals. Moreover, the constraints incorporated in the MPVC method are well followed.

\subsubsection{Dynamic Performance of MPVC Method}

In this subsection, the dynamic performance of the proposed MPVC method is studied based on the hardware platform. $N_{P}, N_{C}, \lambda$ and $T_{S}$ are set as $2,1,0.001$ and $5_{\mu} \mathrm{S}$ respectively.

\subsubsection{Transient Response to Reference Voltage Variation}

The experimental waveforms of transient response to the reference voltage variation are shown in Figure 3-12. It can be seen that the initial values of the reference voltages $v_{R e f, 1}$ and $v_{R e f, 2}$ are $12 \mathrm{~V}$ and $10 \mathrm{~V}$ respectively, and the output voltages are $v_{1}=12.0 \mathrm{~V}$ and 


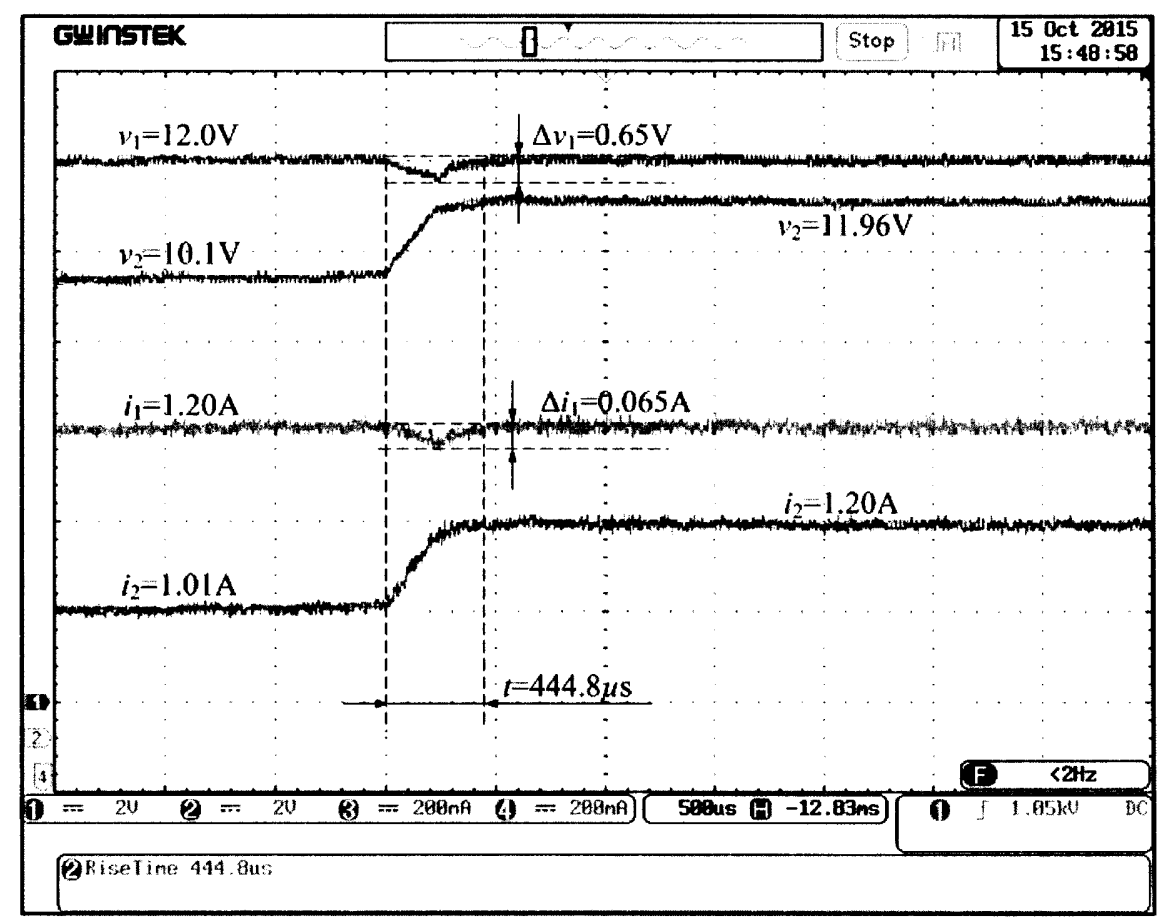

Figure 3-12: Transient response to reference voltage variation waveforms of the proposed MPVC method based on SI-SIDO buck converter. Output voltages $v_{1}, v_{2} \sim 2 \mathrm{~V} / \mathrm{div}$, output currents $i_{1}$, $i_{2} \sim 200 \mathrm{~mA} / \mathrm{div}$, time $\sim 500 \mu \mathrm{s} / \mathrm{div}$.

$v_{2}=10.1 \mathrm{~V}$ which track the reference voltages closely. Then, there is a step change in

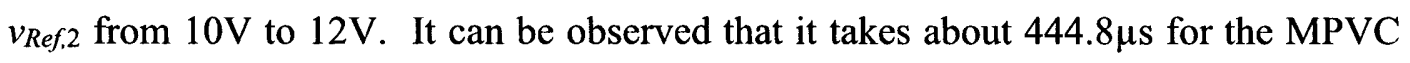
method to regulate $v_{2}$ from $10.1 \mathrm{~V}$ to $11.96 \mathrm{~V}$ to follow the reference change. Correspondingly, the output current $i_{2}$ is increased from 1.01A to $1.20 \mathrm{~A}$. As the load $R_{2}$

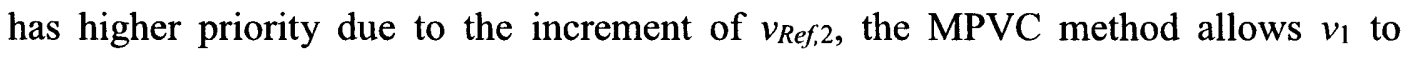
discharge freely for a certain time slot to meet the increasing demand of $V_{2}$. Hence, the waveform of $v_{1}$ has a drop of $\Delta v_{1}$ equal to $0.65 \mathrm{~V}$ due to the step change in $v_{2}$. Accordingly, the output current $i_{1}$ also has a drop of about $0.065 \mathrm{~A}$. However, $v_{1}$ and $i_{1}$ recover quickly to $12.0 \mathrm{~V}$ and $1.20 \mathrm{~A}$ in $444.8 \mu \mathrm{s}$. The transient response waveforms demonstrate that it takes short time for the proposed MPVC method to respond to the reference voltage variation.

\subsubsection{Load Regulation}

The experimental waveforms for load regulation of the proposed MPVC method are shown in Figure 3-13. The two output voltages $v_{1}=12.0 \mathrm{~V}$ and $v_{2}=10.0 \mathrm{~V}$ follow the preset reference voltages $v_{R e f, 1}=12 \mathrm{~V}$ and $V_{R e f, 2}=10 \mathrm{~V}$ successfully. In Figure 3-13(a), load $R_{1}$ is changed to $5 \Omega$ by parallel connecting a resistor of $10 \Omega$ with the previous resistor of $10 \Omega$. Accordingly, the output current $i_{1}$ is increased from $1.20 \mathrm{~A}$ to $2.41 \mathrm{~A}$ 


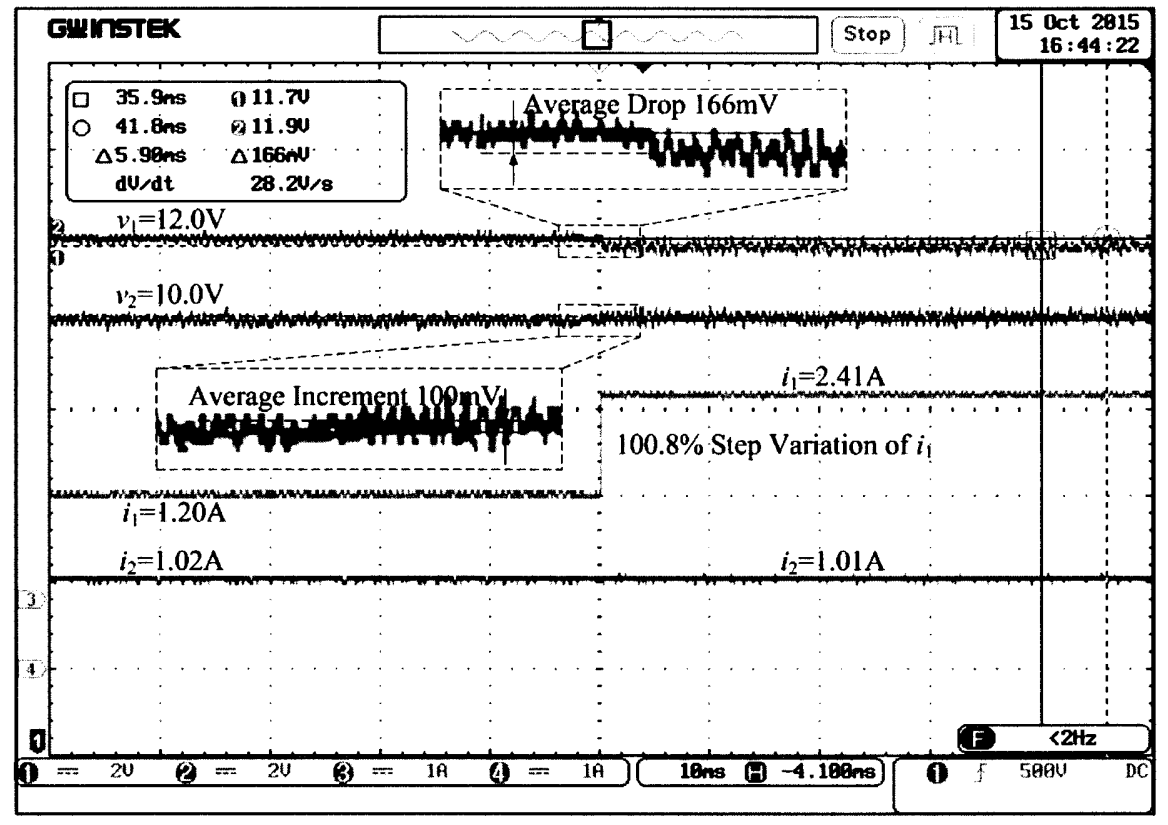

(a)

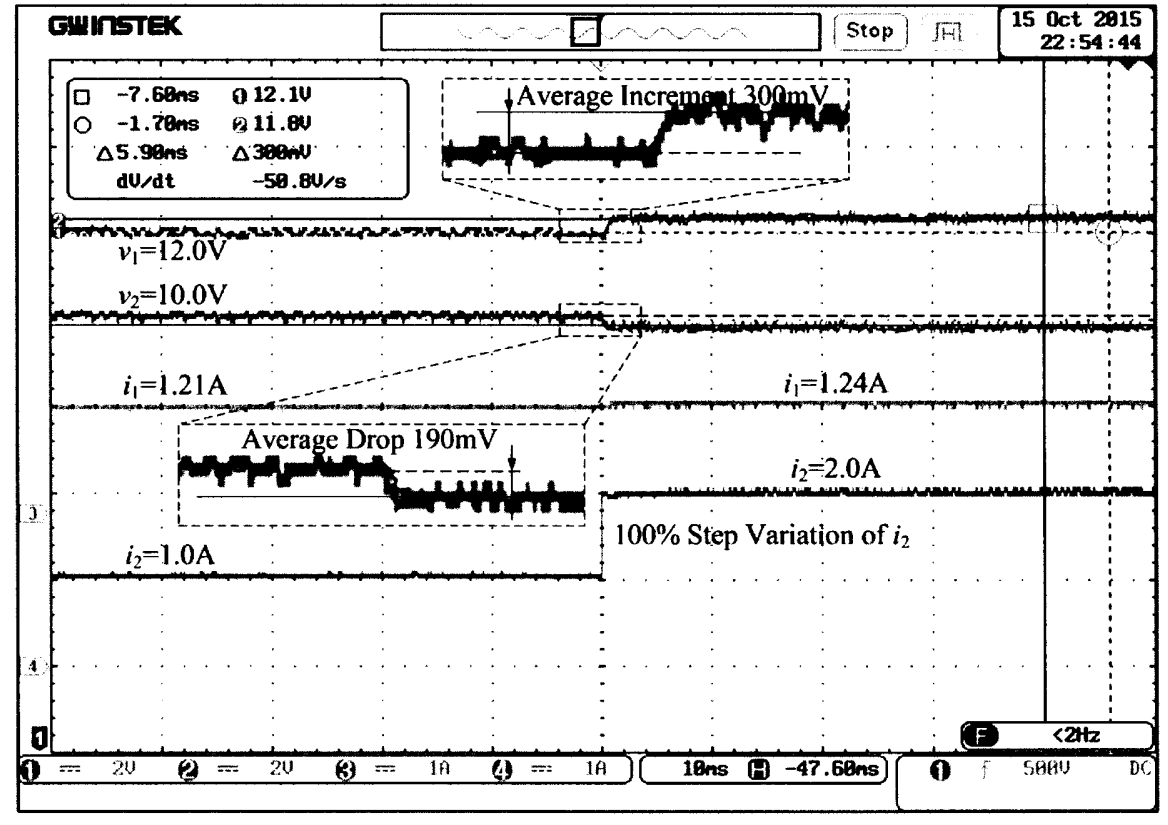

(b)

Figure 3-13: Load regulation waveforms of the proposed MPVC method. $V_{1}, V_{2} \sim 2 \mathrm{~V} / \mathrm{div}, I_{1}$, $I_{2} \sim 1 \mathrm{~A} / \mathrm{div}$, time $\sim 10 \mathrm{~ms} / \mathrm{div}$. (a) Step change in $R_{1}$. (b) Step change in $R_{2}$.

with an increment of $\Delta i_{1}=1.21 \mathrm{~A}$, i.e., $100.8 \%$ increment of the load. It can be observed that there is an average drop of $166 \mathrm{mV}$ in $v_{1}$ and an average increment of $100 \mathrm{mV}$ in $v_{2}$, which are caused by the cross regulation as discussed in [74]. Similarly, load $R_{2}$ is changed to $5 \Omega$. In Figure 3-13(b), it can be seen that the output current $I_{2}$ is increased correspondingly from $1.0 \mathrm{~A}$ to $2.0 \mathrm{~A}$ with an increment of $\Delta i_{2}=1.0 \mathrm{~A}$, i.e., $100 \%$ 


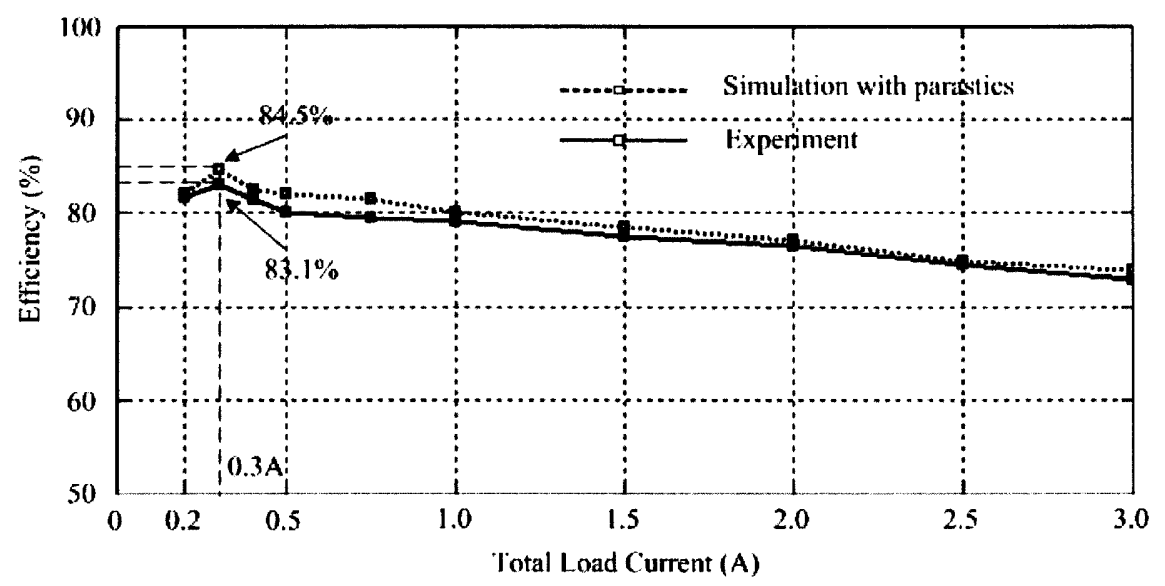

Figure 3-14: Efficiency versus total load current of the proposed MPVC method based on the SIDO buck converter.

increment of the load. The cross regulation results in an average increment of $300 \mathrm{mV}$ in $v_{1}$ and an average drop of $190 \mathrm{mV}$ in $v_{2}$. The experimental results indicate that the dynamic and fast response ability of the proposed MPVC method contributes to the steady voltage regulation with reduced cross regulation. Therefore, the proposed MPVC is able to cope with the step load change.

\subsubsection{Efficiency}

The efficiency of the proposed MPVC method is measured and the efficiency versus the total load current is shown in Figure 3-14. Total load current is the sum of the two output currents $i_{1}$ and $i_{2}$, which ranges from $0.2 \mathrm{~A}$ to $3.0 \mathrm{~A}$. For the simulation efficiency, the parasitic resistances of the inductor and capacitors, and the on-resistances of the MOSFET and diode are all added into the simulation platform based on the corresponding datasheets. It can be seen that the simulation and experimental results correspond well with each other. Moreover, the efficiency has a maximum simulation value of $84.5 \%$ and a maximum experimental value of $83.1 \%$, when the total load current is $0.3 \mathrm{~A}$.

\subsubsection{Comparison with Existing Methods for SI-SIMO DC-DC Converter}

The comparison with the other methods for the SI-SIMO DC-DC converter is shown in Table 3-3. The maximum output power of this work is $39 \mathrm{~W}$, which higher than those of the previous works in the table. This output power level requires that the circuit and controller designed in this paper are for power electronic devices implemented on PCB. Therefore, in this work, the switching frequency is lower and the inductor and capacitors are higher than those in the table, but similar to [77], [116]. 
Table 3-3 Comparison with Existing Methods for SIMO DC-DC Converter

\begin{tabular}{|c|c|c|c|c|c|}
\hline Reference & [71] & [72] & [74] & [75] & This Work \\
\hline Process & FPGA & $0.25 \mu \mathrm{m}$ CMOS & Discrete Components & FPGA & $\begin{array}{c}\text { Discrete } \\
\text { Components }\end{array}$ \\
\hline Input Voltage & $2.5-5 \mathrm{~V}$ & $2.8-5 \mathrm{~V}$ & $4.8 \mathrm{~V}$ & $5 \mathrm{~V}$ & $20 \mathrm{~V}$ \\
\hline $\begin{array}{c}\text { Output } \\
\text { Voltage }\end{array}$ & $0.9,1.5 \mathrm{~V}$ & $1.2,1.8 \mathrm{~V}$ & $3.3,1.2 \mathrm{~V}$ & $1,1.5 \mathrm{~V}$ & $12,8-12 V$ \\
\hline Load Current & $0.5,0.5 \mathrm{~A}$ & $0.4,0.2 \mathrm{~A}$ & $0.2,0.1 \mathrm{~A}$ & $0.5,0.5 \mathrm{~A}$ & $1.2-2.4,0.8-2.0 \mathrm{~A}$ \\
\hline Output Power & $1.2 \mathrm{~W}$ & $0.84 \mathrm{~W}$ & $0.78 \mathrm{~W}$ & $1.25 \mathrm{~W}$ & $39 \mathrm{~W}$ \\
\hline $\begin{array}{l}\text { Control } \\
\text { Method }\end{array}$ & PWM & $\begin{array}{c}\text { Multivariable } \\
\text { control based } \\
\text { PWM } \\
\end{array}$ & $\begin{array}{c}\text { Cross-derivative state } \\
\text { feedback based } \\
\text { PWM }\end{array}$ & PWM and PFM & MPVC \\
\hline$f_{s w}$ & $500 \mathrm{kHz}$ & $600 \mathrm{kHz}$ & $100 \mathrm{kHz}$ & $500 \mathrm{kHz}$ & $20-100 \mathrm{kHz}$ \\
\hline$L$ & $5 \mu \mathrm{H}$ & $4.7 \mu \mathrm{H}$ & $10 \mu \mathrm{H}$ & $5 \mu \mathrm{H}$ & $100 \mu \mathrm{H}$ \\
\hline C & $10 \mu \mathrm{F}$ & $22 \mu \mathrm{F}$ & $10 \mu \mathrm{F}$ & $10 \mu \mathrm{F}$ & $100 \mu \mathrm{F}$ \\
\hline $\begin{array}{l}\text { FOM } \\
\text { (self) }\end{array}$ & 0.04 & 0.04 & 0.03 & 0.03 & 0.014 \\
\hline $\begin{array}{c}\text { FOM } \\
\text { (cross) }\end{array}$ & 0.027 & 0.027 & 0.008 & 0.01 & 0.019 \\
\hline
\end{tabular}

FOM (self): (Overshoot in output voltage in self channel / Rated voltage) / (Load step in self channel / Rated load).

FOM (cross): (Overshoot in output voltage in cross channel / Rated voltage) / (Load step in cross channel / Rated load).

Two performance indices FOM (self) and FOM (cross) have been defined in [74], [75] to estimate the performance of existing methods for the SI-SIMO DC-DC converter, as shown at the bottom of Table 3-3. Based on the definitions and the experimental results in Figure 3-13, the two performance indices FOM (self) and FOM (cross) are calculated as 0.014 and 0.019 respectively. Compared to the existing methods, the MPVC method has the FOM (self) value of 0.014 , which is less than all existing methods, and the FOM (cross) value of 0.019, which is less than [71], [72], however, greater than [74], [75]. Therefore, the proposed method has the best cross regulation suppression on self-channel and medium cross regulation suppression on cross-channel. Although the FOM (cross) in this paper is greater than [74], [75], the maximum efficiency of $83.1 \%$ in this work is greater than that of $80 \%$ in [74], and the proposed MPVC method is able to regulate the output voltages independently, however, the output voltage of one channel is always less than the other in [75]. In summary, the proposed MPVC method is a promising method to control the SI-SIMO DC-DC converter.

The MPVC method has a variable switching frequency by generating the control signal directly. Based on (3.21) and (3.22), the switching frequency plays a critical role in the selection of inductor and capacitors. Therefore, a better DSP controller with higher switching frequency will reduce the values of inductor and capacitors. 
Considering the power level of this implementation and the values of FOM (self) and FOM (cross), the proposed MPVC method is a promising method to control SI-SIMO DC-DC converter.

\subsection{Conclusion}

In this chapter, the MPVC method to reduce the cross regulation for the SI-SIMO DC-DC converter has been presented. The state-space model of the SI-SIMO buck converter for the MPVC method has been developed with corresponding cost function and constraints. Based on the state-space model, cost function and constraints, the MPVC controller for the SI-SIMO DC-DC converter can generate the optimal control signals so that the cross regulation is suppressed significantly. Moreover, the design of the values of inductor and capacitors is also analyzed for the MPVC method.

Simulation and experimental test cases have been conducted to study the performance of the proposed MPVC method. In the simulation, the influences of the two MPC parameters control horizon $N_{C}$ and Lagrange multiplier $\lambda$ on the voltage ripple, the steady-state operation and the dynamic performance of the proposed MPVC method have been studied to guide the hardware implementation. Finally, a hardware platform of the SI-SIDO buck converter has been built, and several experimental test cases have been presented to verify the performance of the proposed MPVC method including steady-state operation and dynamic performance. The simulation and experimental results have demonstrated that the voltage ripples are regulated less than $5 \%$ in steadystate operation, and the MPVC method responds to the step change of output voltage reference and the step change of load fast. Moreover, the FOM (self) and FOM (cross) of the proposed MPVC method are 0.014 and 0.019 , which are better than most existing control method for SI-SIMO DC-DC converter. Therefore, the proposed MPVC method can regulate the SI-SIMO DC-DC converter successfully with reduced cross regulation, and respond fast to the step change in load and reference. 


\section{CHAPTER 4 MODEL PREDICTIVE CURRENT CONTROL FOR CHARGING EQUALIZATION BASED ON SI-SIMO DC-DC CONVERTER}

In this chapter, a model predictive current control (MPCC) method is developed for SI-SIMO DC-DC converter. Based on the MPCC method, a charging equalization method is proposed for the series-battery in EVs.

\subsection{MPCC METHOD}

\subsubsection{Architecture of SI-SIMO DC-DC Buck Converter for Charging Equalization}

The buck converter is taken as the example to explain the proposed method. The charge equalization system architecture is shown in Figure 4-1. $V_{d c}$ is the DC voltage source with an internal resistance $R_{s}$ to charge the battery cells. $V$ is the voltage across the freewheeling diode $D$ cathode and the ground. $S$ is the switch between the DC source $V_{d c}$ and the inductor $L . i_{L}$ is the current passing through the inductor $L$, which has an ESR $R_{L} . i_{i}$ and $v_{i}$ are the corresponding current and voltage of each battery cell $B_{i}$ respectively. $S_{i}$ is the switch used to select the $i$ th charging branch. $i$ has a value from 1 to $n$, where $n$ is the number of battery cells. The value of $S$ or $S_{i}$ is equal to 1 when the switch is on. Otherwise, it is equal to 0 .

The SI-SIMO DC-DC buck converter individually charges battery cells considering their respective SOC. Thus, the system balances all battery cells during the charging process. Moreover, the system reduces the number of required components and cost because the converter uses a single inductor to realize the individual charging operation. However, due to the cells sharing the same inductor, the reduction of the mutual interference (i.e., cross regulation problem) is critically considered.

\subsubsection{MPCC Method}

As mentioned above, the MPCC method is proposed to control the SI-SIMO DC-DC buck converter for the individual charging operation. Therefore, the development of the state-space model for prediction is imperative. The MPCC method is a timemultiplexing method, so that it involves only one battery cell $B_{i}$ connected to the inductor $L$ during each charging interval. Therefore, a single-output buck converter is 


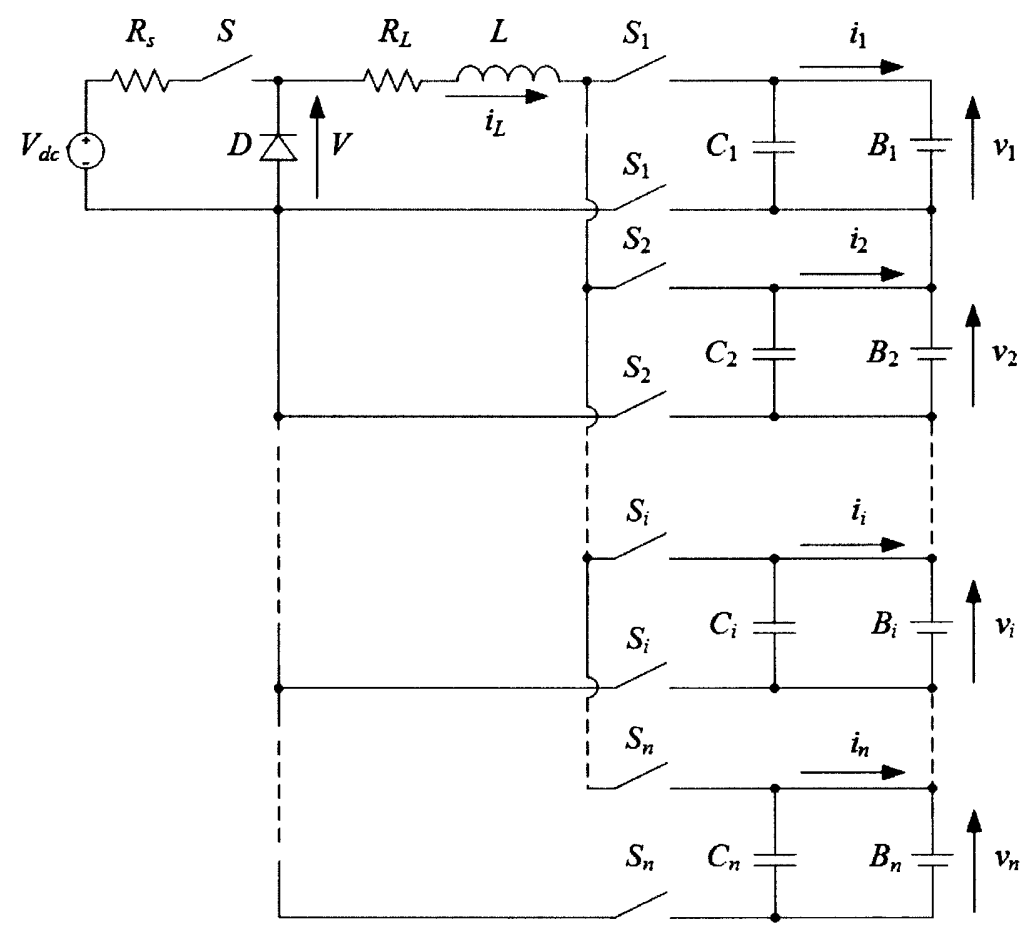

Figure 4-1: Battery charging equalization system architecture based on the SI-SIMO DC-DC buck converter.

formed when individual charging is conducted. Consequently, the state-space model for prediction is developed based on the single-output buck converter.

The $i$ th battery cell $B_{i}$ is taken as the example for the predictive model control development. $B_{i}$ is connected to the inductor $L$ by closing the switch $S_{i}$. Hence $S_{i}$ is switched on for selecting $B_{i}$. To establish the model, the characteristic differential equations of inductor $L$ and capacitor $C_{i}$ of $B_{i}$ are expressed as

$$
\begin{gathered}
L \frac{d i_{L}}{d t}=V-v_{i}-i_{L} R_{L} \\
C_{i} \frac{d v_{i}}{d t}=i_{L}-i_{i}
\end{gathered}
$$

Moreover, the relationship between $V$ and $V_{d c}$ is given by

$$
V=S V_{d c}
$$

where $S=1$ when it is on, and $S=0$ when it is off, and $R_{S}$ is neglected since its value is small compared to other resistances.

By substituting (4.3) into (4.1), it can be obtained as

$$
L \frac{d i_{L}}{d t}=S V_{d c}-v_{i}-i_{L} R_{L}
$$


Given that the MPCC method is operated in discrete time with sampling time $T_{s}$, the continuous equations (4.2) and (4.4) are converted into a discrete form. For (4.2), the discrete form when $t$ is equal to $k$ can be expressed as

$$
v_{i}(k+1)-v_{i}(k)=\frac{T_{S}}{C_{i}}\left(i_{L}(k)-i_{i}(k)\right)
$$

The left side of (4.5) is defined as $\Delta v_{i}(k+1)$, i.e., the increment of $v_{i}(k)$. Thus, (4.5) can be rewritten as

$$
i_{i}(k)=i_{L}(k)-\frac{C_{i}}{T_{S}} \Delta v_{i}(k+1)
$$

Similarly, the discrete form of (4.4) when $t$ is equal to $k$ is

$$
i_{L}(k+1)-i_{L}(k)=\frac{T_{S}}{L}\left(S(k) V_{d c}-v_{i}(k)-i_{L}(k) R_{L}\right)
$$

Therefore, the discrete form of (4.4) when $t=k-1$ can be expressed as

$$
i_{L}(k)-i_{L}(k-1)=\frac{T_{S}}{L}\left(S(k-1) V_{d c}-v_{i}(k-1)-i_{L}(k-1) R_{L}\right)
$$

Using (4.7) to subtract (4.8), it can be obtained as

$$
\Delta i_{L}(k+1)=\left(1-\frac{R_{L} T_{S}}{L}\right) \Delta i_{L}(k)+\frac{T_{S}}{L}\left(\Delta S(k) V_{d c}-\Delta v_{i}(k)\right)
$$

where $\Delta i_{L}(k)$ and $\Delta S(k)$ are defined as the increment of $i_{L}(k)$ and $S(k)$ respectively.

Similarly, the expression of (4.6) in the next time step is

$$
i_{i}(k+1)=i_{L}(k+1)-\frac{C_{i}}{T_{s}} \Delta v_{i}(k+2)
$$

Subtracting (4.6) from (4.10), it can be obtained as

$$
i_{i}(k+1)-i_{i}(k)=\Delta i_{L}(k+1)-\frac{C_{i}}{T_{S}} \Delta v_{i}(k+2)+\frac{C_{i}}{T_{S}} \Delta v_{i}(k+1)
$$

By substituting (4.9) into (4.11) and assuming that $\Delta v_{i}(k+2)=\Delta v_{i}(k+1)$ under a high sample frequency, $i_{i}(k+1)$ is expressed as

$$
i_{i}(k+1)=i_{i}(k)+\left(1-\frac{R_{L} T_{S}}{L}\right) \Delta i_{L}(k)+\frac{T_{S}}{L} \Delta S(k) V_{d c}-\frac{T_{S}}{L} \Delta v_{i}(k)
$$

Based on (4.9) and (4.12), the augmented discrete-time state-space model for the MPCC method can be obtained as

$$
\begin{aligned}
& x(k+1)=A x(k)+B \Delta S(k)+C \Delta v_{i}(k) \\
& y(k)=D x(k)
\end{aligned}
$$




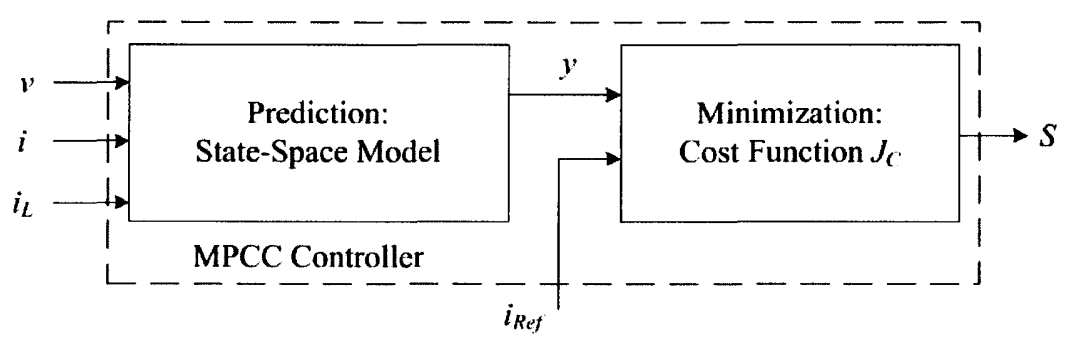

Figure 4-2: Block diagram of the MPCC controller structure.

where $x(k)=\left[\Delta i_{L}(k) i_{i}(k)\right]^{T}$ is the state vector of the single buck converter, and $y(k)=i_{i}(k)$ is the output current of the single buck converter. The coefficient matrices are listed as follows:

$$
A=\left[\begin{array}{ll}
1-R_{L} T_{S} / L & 0 \\
1-R_{L} T_{S} / L & 1
\end{array}\right] ; B=\left[\begin{array}{ll}
T_{S} V_{d c} / L & T_{S} V_{d c} / L
\end{array}\right]^{T} ; C=\left[\begin{array}{ll}
-T_{S} / L & -T_{S} / L
\end{array}\right]^{T} ; D=\left[\begin{array}{ll}
0 & 1
\end{array}\right]
$$

The discrete-time state-space model and the related data $\left(v_{i}, i_{i}, i_{L}\right)$ can be used to predict all the possible future output currents $y$ from the corresponding control signals. Based on the MPC theory, the predict and control horizons and the Lagrange multiplier are defined as $N_{P}, N_{C}$ and $\lambda$ respectively. The cost function $J_{C}$ for the MPCC method is defined as

$$
J_{C}=\sum_{j=1}^{N_{P}}\left|y(k+j)-i_{R e f i}(k+j)\right|+\lambda \sum_{j=1}^{N_{C}}|\Delta S(k+j-1)|
$$

where $i_{R e f, i}$ is the current reference for $B_{i}$. Moreover, the quadratic programming algorithm aids in determining the optimal control actions $S$, which are able to minimize $J_{C}$. An advantage of MPC is that the operating constraints can be easily incorporated into the controller design. In this thesis, a constraint is imposed on the inductor current $i_{L}$ to prevent overshooting when there is a sudden change in the reference.

The MPCC controller structure is shown in Figure 4-2. The input data $\left(v, i, i_{L}, i_{R e f}\right)$ of the controller is updated by the corresponding data $\left(v_{i}, i_{i}, i_{L}, i_{R e f, i}\right)$ if the $i$ th battery cell $B_{i}$ is presently connected to the inductor $L$ for individual charging. It can be seen that the controller has two main parts: prediction block and minimization block. In the prediction block, $\left(v, i, i_{L}\right)$ can be used to predict all possible future results $y$ based on the state-space model. $y$ and $i_{R e f}$ are transmitted to the minimization block to determine the optimal switching control action $S$. Hence, the output current $i$ effectively tracks $i_{R e f}$. 


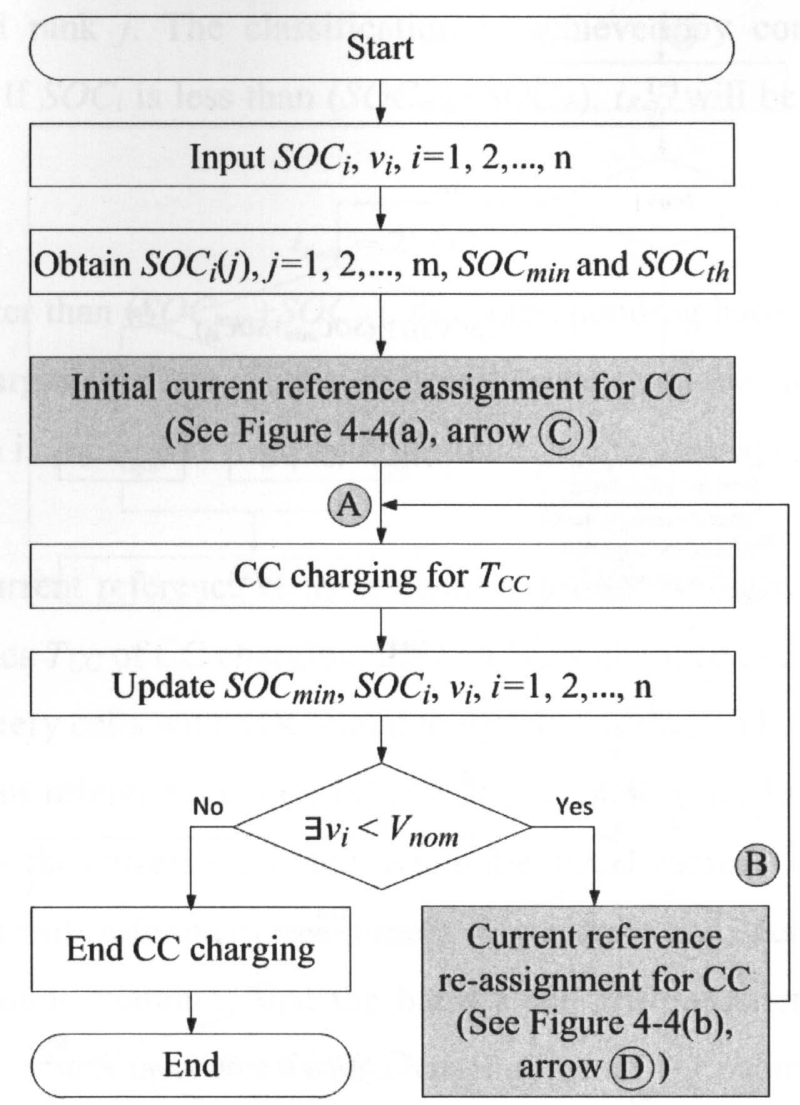

Figure 4-3: CC charging flowchart. A and B are the symbols of the related arrows. Initial current reference assignment algorithm for $\mathrm{CC}$ and current reference re-assignment algorithm for $\mathrm{CC}$ are continued in Figure 4-4.

\subsection{Charging Equalization Method}

For the MPCC method, it is important to properly assign the current reference $I_{R e f, i}$ of $B_{i}$. The battery cell is assumed to have the nominal capacity $C_{n o m}$, which determines the maximum charging current $1 \mathrm{C}$, and $S O C_{i}$ denotes the SOC of the battery cell $B_{i}$. The current reference assignment algorithms for $\mathrm{CC}$ and $\mathrm{CV}$ charging are different and discussed below.

\subsubsection{Algorithm for CC Charging}

The CC charging flowchart is shown in Figure 4-3. At the beginning, the SOC data are ranked with label $j$. Therefore, the updated $S O C_{i}(j)$ are obtained, where integer $j$ is from 1 to $\mathrm{m} . \mathrm{m}$ is less than or equal to the battery cell number $\mathrm{n}$ because of the rule for ranking, which is defined as follows:

$$
\left\{\begin{array}{l}
j_{i 1}=j_{i 2}, \text { if } S O C_{i 1}=S O C_{i 2} \\
j_{i 1}>j_{i 2}, \text { if } S O C_{i 1}>S O C_{i 2}
\end{array}\right.
$$




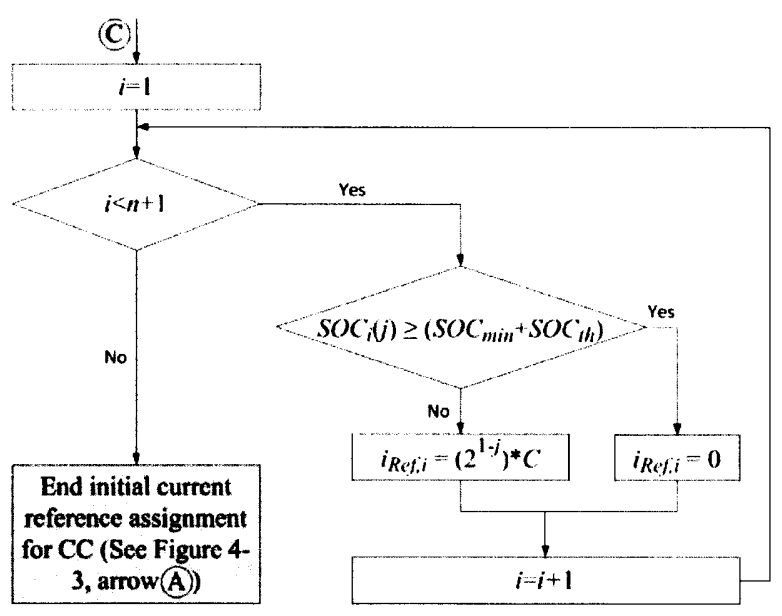

(a)

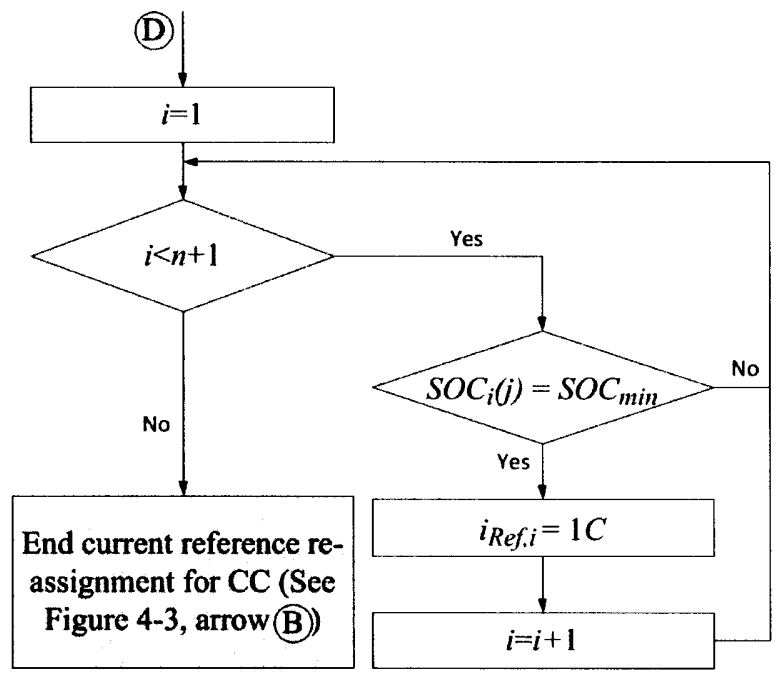

(b)

Figure 4-4: (a) Initial current reference assignment algorithm for CC charging. (b) Current reference re-assignment algorithm for $\mathrm{CC}$ charging. $\mathrm{C}$ and $\mathrm{D}$ are the symbols of the related arrows.

Moreover, $S O C_{\min }$ and a threshold $S O C_{t h}$ are defined to classify the SOCs. After all SOCs are ranked, the initial current references can be assigned properly (the first shadowed block in Figure 4-3). A CC charging cycle $T_{C C}$ is defined for CC charging. When all battery cells finish one charging cycle $T_{C C}, S C O_{\min }, S O C_{i}$ and $v_{i}$ are updated. As long as there is one cell voltage less than the nominal voltage $V_{n o m}=5.8 \mathrm{~V}$, current reference re-assignment (the second shadowed block in Figure 4-3) will be conducted. With the re-assigned current references, the $\mathrm{CC}$ charging continues. The $\mathrm{CC}$ charging and current reference re-assignment will be repeated for several cycles $T_{C C}$ until all $v_{i}$ reach $V_{\text {nom }}$, so that $\mathrm{CC}$ charging is ended.

The detailed initial current reference assignment algorithm is shown in Figure 4-4(a). It can be seen that the different current references are determined with consideration of 
classification and rank $j$. The classification is achieved by comparing $S O C_{i}$ and $\left(S O C_{\min }+S O C_{t h}\right)$. If $S O C_{i}$ is less than $\left(S O C_{\min }+S O C_{t h}\right), i_{R e f, i}$ will be assigned the value as follows:

$$
i_{\text {Ref. } i}=2^{1-j} C
$$

If $S O C_{i}$ is greater than $\left(S O C_{\min }+S O C_{t h}\right)$, the corresponding battery cell $B_{i}$ is isolated during the $\mathrm{CC}$ charging process. That is, $i_{R e f, i}$ will be assigned the value as 0 . Therefore, the battery cell $B_{i}$ is prevented from entering the CV charging stage earlier than other battery cells.

The detailed current reference re-assignment algorithm is shown in Figure 4-4(b). After several cycles $T_{C C}$ of CC charging, the $S O C_{\min }$ is changed and updated, as well as the number of battery cells with SOC equal to updated $S O C_{\min }$. The re-assignment only updates the current reference $i_{R e f, i}$ as $1 \mathrm{C}$ if $S O C_{i}$ is equal to updated $S O C_{\min }$. For the other battery cells, the current references retain the initial values.

The initial current reference assignment algorithm and current reference reassignment algorithm guarantee that the battery cell with smaller SOC has a larger current reference, which indicates faster charging speed. By contrast, the battery cell with larger SOC has a smaller current reference. Consequently, the imbalance of SOC will be gradually eliminated because of the difference in current references.

\subsubsection{Algorithm for CV Charging}

The CV charging flowchart is shown in Figure 4-5(a). All $v_{i}$ reach $V_{n o m}$ when CC charging is completed. If the current references remain unchanged, all $v_{i}$ will exceed $V_{n o m}$. Therefore, the current reference is gradually decreased to keep $v_{i}$ tracking $V_{\text {nom }}$ in further $\mathrm{CV}$ charging process. Similarly, a $\mathrm{CV}$ charging cycle is defined as $T_{C V}$. When all battery cells finish one charging cycle $T_{C V}, S O C_{i}$ and $v_{i}$ are updated. As long as there is one SOC less than 1, the current reference assignment for CV charging (the shadowed block in Figure 4-5(a)) will be conducted to reduce the current references gradually. The CV charging and current reference assignment will be repeated for several cycles $T_{C V}$ until all $S O C_{i}$ reach 1, so that $\mathrm{CV}$ charging is ended.

The detailed current reference assignment algorithm for $\mathrm{CV}$ charging is shown in Figure 4-5(b). It can be seen that $i_{R e f, i}$ has a small decrement every time $v_{i}$ reaches or exceeds $V_{n o m}$, and the decrement of $i_{\text {Ref. } i}$ is set as $0.025 \mathrm{C}$ in the simulation. Actually, $v_{i}$ has a small drop because of the sudden $0.025 \mathrm{C}$ drop of $i_{R e f, i}$. However, $v_{i}$ will reach or exceed $V_{\text {nom }}$ again after several $T_{C V}$ of $\mathrm{CV}$ charging, so that the current reference is 


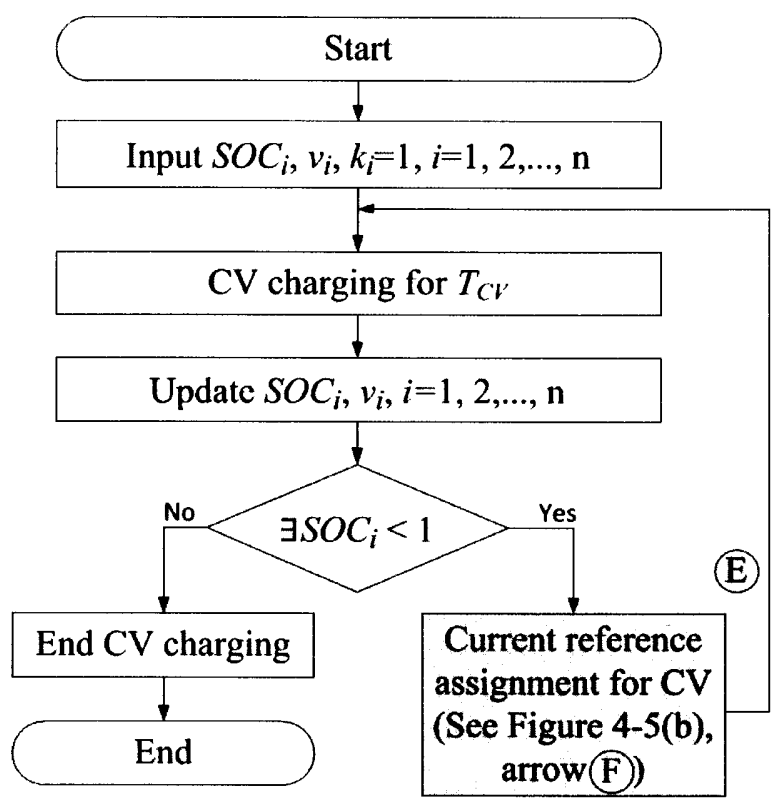

(a)

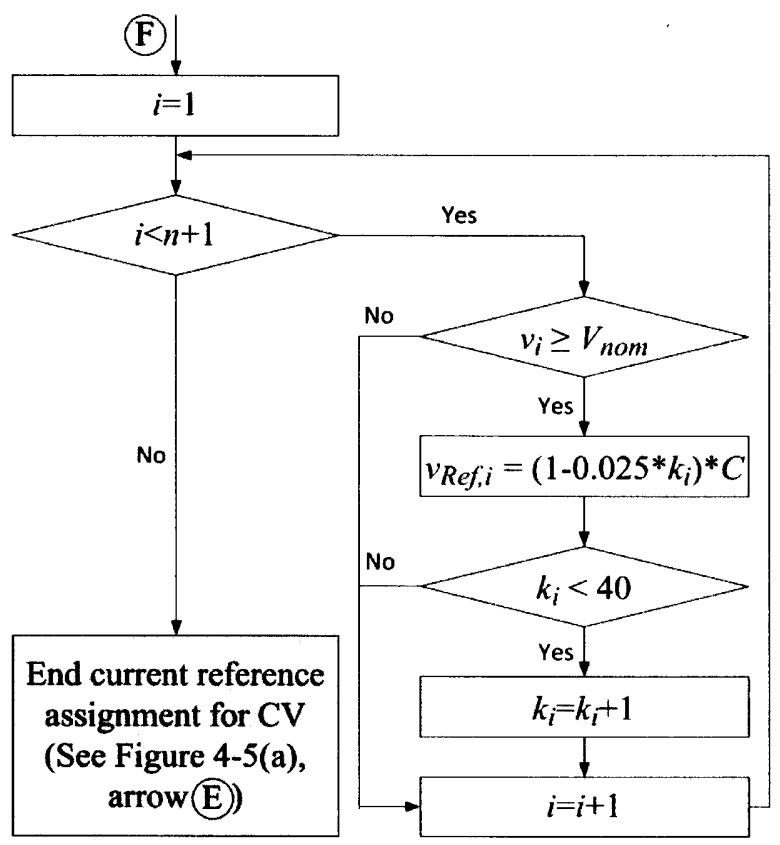

(b)

Figure 4-5: (a) CV charging flowchart. (b) Current reference assignment algorithm for CV charging. $\mathrm{E}$ and $\mathrm{F}$ are the symbols of the related arrows.

again decreased by $0.025 \mathrm{C}$. This repeated process will guarantee all $v_{i}$ track $V_{\text {nom }}$ closely. Notice that the lower limit of current reference is set as $0.025 \mathrm{C}$, which results from the upper limit of $k_{i}$ set as 39 , i.e., the lowest charging current is $0.025 \mathrm{C}$. 


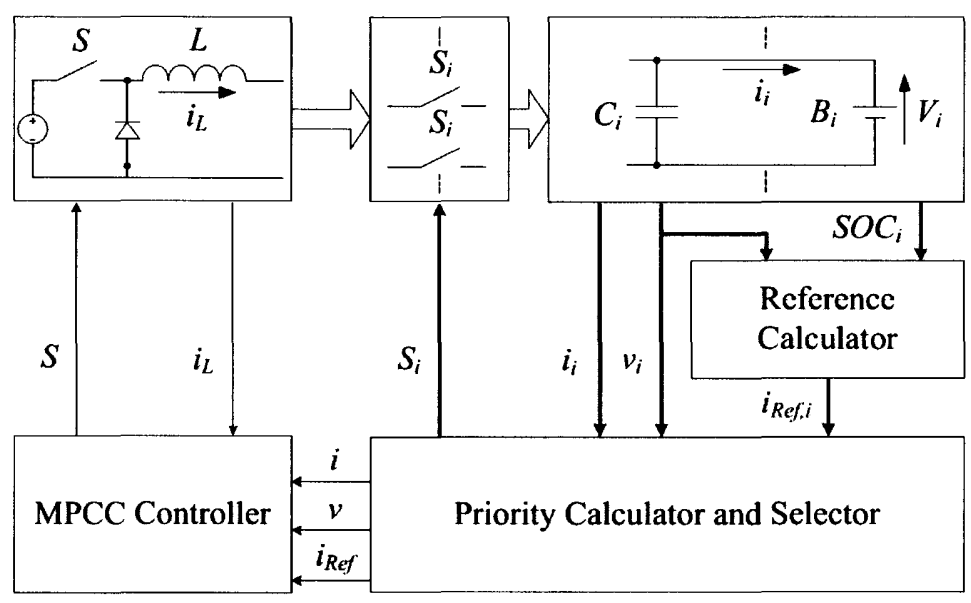

Figure 4-6: SI-SIMO DC-DC buck converter cortroller for charge equalization.

\subsubsection{Charge Equalization Method}

The charge equalization method is developed based on the MPCC method and the current reference assignment algorithms. The MPCC method exhibits a dynamic and fast response. Hence, the SI-SIMO DC-DC buck converter is capable of supporting different current outputs when tracking the pre-set current references with significantly reduced cross regulation.

The charge equalization controller is presented in Figure 4-6. The $v_{i}$ and $S O C_{i}$ values of all battery cells are measured and sent to the reference calculator block, and the current reference assignment algorithms for $\mathrm{CC}$ or $\mathrm{CV}$ charging will be executed. The priority calculator generates the priority $p_{i}$ of each battery cell based on the data $\left(v_{i}, i_{i}\right.$, $\left.i_{R e f, i}\right)$ after all current references are determined. The Hunger algorithm is adopted for the priority calculation as shown in (4.17). It can be seen that a larger difference between the charging current $I_{i}$ and the current reference $i_{R e f, i}$ results in a higher priority $p_{i}$ value.

$$
p_{i}=\left|i_{i}-i_{\text {Refi. }}\right|
$$

The priority information aids the selector in selecting the battery cell $B_{i}$ by setting the corresponding switch $S_{i}$ as 1 and the other switches as 0 . The selected data $\left(v_{i}, i_{i}, i_{\text {Ref }, i}\right)$ are then updated into the MPCC controller as $\left(v, i, i_{R e f}\right)$ respectively. The current of inductor $i_{L}$ is also updated. The MPCC controller will generate the optimal switching control action $S$ to regulate the converter. Thus, the selected $B_{i}$ is charged with the current $i_{i}$, which effectively tracks $i_{R e f, i}$. 
Table 4-1 Simulation parameters

\begin{tabular}{c|c|c|c|c|c|c|c}
\hline \hline Symbol & $V_{d c}$ & $L$ & $R_{L}$ & $C_{i}$ & $T_{S}$ & $N_{P}$ & $N_{C}$ \\
\hline Value & $15 \mathrm{~V}$ & $500 \mu \mathrm{H}$ & $0.14 \Omega$ & $500 \mu \mathrm{F}$ & $2 \mu \mathrm{s}$ & 12 & 4 \\
\hline Symbol & $\lambda$ & $C_{n o m}$ & $V_{n o m}$ & $S O C_{t h}$ & $T_{C C}$ & $T_{C V}$ & \\
\hline Value & 0.001 & $0.4 \mathrm{Ah}$ & $5.8 \mathrm{~V}$ & 0.1 & $10 \mathrm{~ms}$ & $10 \mathrm{~ms}$ & \\
\hline
\end{tabular}

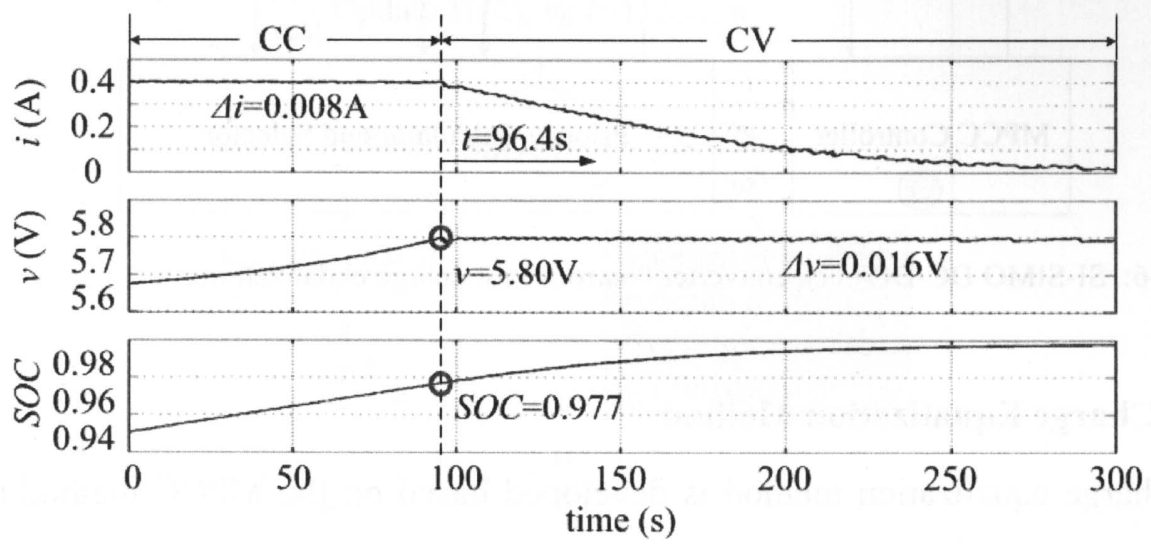

Figure 4-7: CC charging and CV charging waveforms based on the MPCC method.

\subsection{SimUlation STUdiES}

The charge equalization system based on the SI-SIMO DC-DC buck converter is built in MATLAB/Simulink. The simulation results are presented and discussed in this section. The lithium-ion battery model in Matlab/Simulink is adopted for the simulation studies. The battery parameters and the simulation parameters are listed in Table 4-1. The capacity $C_{n o m}$ of the battery cell is scaled down to $0.4 \mathrm{Ah}$ for simplification.

\subsubsection{Charging and CV Charging Based on MPCC Method}

The capability of the MPCC method to regulate the SI-SIMO DC-DC buck converter to achieve the $\mathrm{CC}$ and $\mathrm{CV}$ charging processes is studied in this section. A single battery cell with an initial SOC of 0.95 is selected for the charging process.

The simulation results are shown in Figure 4-7, and the charging process is divided into CC stage and CV stage. During CC stage, the current for the CC charging is initially set as $0.4 \mathrm{~A}(1 \mathrm{C})$. To evaluate the performance of $\mathrm{CC}$ charging, an average current ripple $\Delta i$ is defined, which is the average difference between $i$ and $i_{R e f}$. It can be seen from Figure 4-7 that $\Delta i$ is $0.008 \mathrm{~A}$, which is $2 \%$ of the current reference. With the CC charging, the voltage and the SOC are gradually increased. The SOC is increased to 0.977 , and the voltage reaches $V_{n o m}=5.8 \mathrm{~V}$ when $t=96.4 \mathrm{~s}$. Therefore, the $\mathrm{CC}$ charging stage is over 

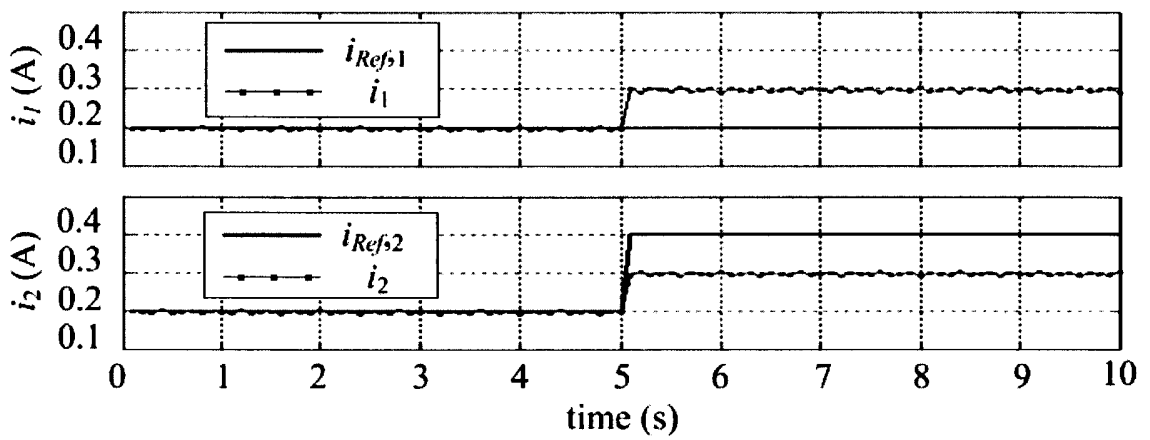

(a)
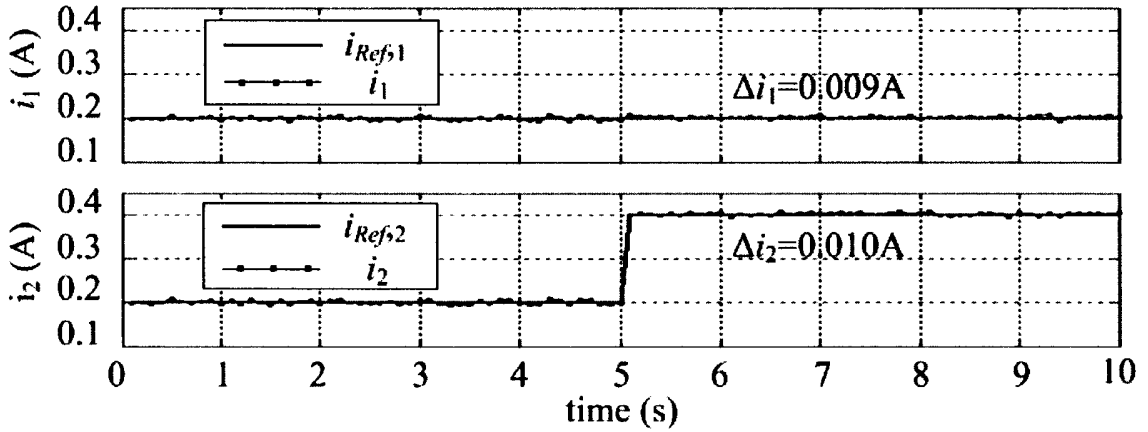

(b)

Figure 4-8: Comparison of the output currents without and with the MPCC method based on SI-SIDO buck converter. (a) A typical cross regulation phenomenon without the MPCC method. (b) Simulation results based on the MPCC method.

and CV charging is executed. CV charging is conducted by gradually decreasing the charging current from $0.4 \mathrm{~A}(1 \mathrm{C})$ to $0.01 \mathrm{~A}(0.025 \mathrm{C})$ with $0.01 \mathrm{~A}$ decrement. The current decrease happens every time when the voltage reaches or exceeds $V_{\text {nom }}$. The small current decrease leads to the sudden small voltage drop. Therefore, the voltage is maintained at approximately to $V_{\text {nom }}$ with a tolerable average voltage ripple $\Delta v=0.016 \mathrm{~V}$ (3\% of $V_{n o m}$ ), which is the average difference between $V$ and $V_{n o m}$. CV charging is completed when the battery cell is fully charged.

The simulation results show that the MPCC method steadily regulates the output current and voltage in the $\mathrm{CC}$ and $\mathrm{CV}$ charging stages respectively. Therefore, the proposed method effectively implements the $\mathrm{CC}$ and $\mathrm{CV}$ charging processes.

\subsubsection{MPCC for SI-SIMO DC-DC Buck Converter}

Because of the structure of the SI-SIMO DC-DC buck converter, all battery cells share the same inductor. Moreover, the current reference sent into the MPCC controller varies based on the priority $p_{i}$. Consequently, there is a cross regulation problem, which means that the mutual influence among all battery cells will lead the output current of 


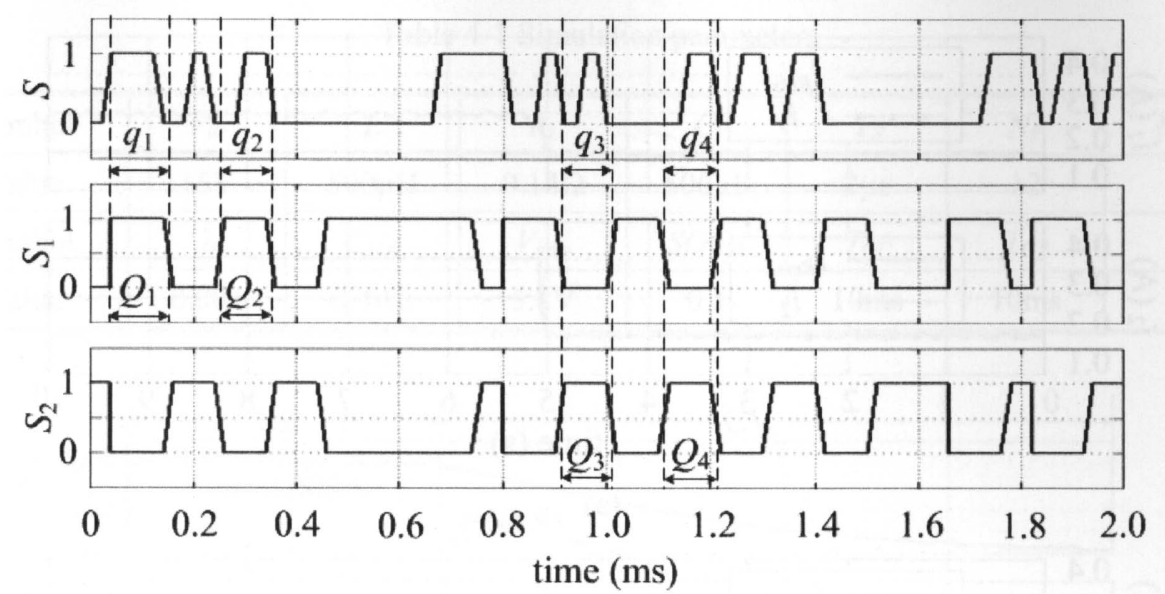

Figure 4-9: Control signals for the SI-SIMO DC-DC buck converter with MPCC.

each branch fail to track the related current reference. The performance of the proposed MPCC method in terms of reduction in cross regulation is verified in this section. For simplification, the number of battery cells $n$ is set as 2, which indicates that there are two battery cells.

A typical cross regulation phenomenon is shown in Figure 4-8(a). At the beginning, the two references $i_{R e f, 1}$ and $i_{R e f, 2}$ are both set as $0.2 \mathrm{~A}$, the SI-SIMO buck converter works well. When $t=5 \mathrm{~s}, i_{R e f, 2}$ is changed to $0.4 \mathrm{~A}$, and $i_{R e f, 1}$ is unchanged. Due to the mutual interference, $i_{1}$ is pulled up around $0.3 \mathrm{~A}$ and $i_{2}$ is also stuck around $0.3 \mathrm{~A}$. Therefore, the cross regulation happens when the references are different, and the cross regulation seriously deteriorates the individual charging performance. Solving the cross regulation problem is critical for controlling the SI-SIMO DC-DC buck converter.

The proposed MPCC method is applied to control the SI-SIMO DC-DC buck converter, and the results are shown in Figure 4-8(b). Similarly, $i_{R e f, 1}$ is kept as $0.2 \mathrm{~A}$ and $i_{R e f, 2}$ is changed from $0.2 \mathrm{~A}$ to $0.4 \mathrm{~A}$ when $t=5 \mathrm{~s}$. It can be seen that $i_{1}$ and $i_{2}$ closely track the current references $i_{R e f, 1}$ and $i_{R e f, 2}$ respectively. In detail, the average current ripples $\Delta i_{1}$ and $\Delta i_{2}$ are $0.009 \mathrm{~A}$ and $0.010 \mathrm{~A}$ respectively, which means that the cross regulation is solved.

To explain how the proposed method solves the cross regulation problem, some extracted parts of the control signals $S, S_{1}$ and $S_{2}$ are shown in Figure 4-9. Two adjacent pulse-widths of $S_{1}$ are $Q_{1}$ and $Q_{2}$ respectively, when $S_{1}$ is switched on (i.e., the priority $p_{1}>p_{2}$ and $B_{1}$ is selected). During $Q_{1}$ and $Q_{2}$, the corresponding two pulse-widths of $S$ are $q_{1}$ and $q_{2}$ respectively. It can be observed that the duty cycles of $q_{1}$ and $q_{2}$ are 
different, which means the MPCC controller adjusts the control signal $S$ based on the real-time data $\left(v_{1}, i_{1}, i_{L}, i_{R e f, 1}\right)$. Similarly, the pulse-widths $q_{3}$ and $q_{4}$ of $S$ also dynamically response to the real-time data $\left(v_{2}, i_{2}, i_{L}, i_{R e f, 2}\right)$, during the pulse-widths $Q_{3}$ and $Q_{4}$ when $S_{2}$ is on (i.e., the priority $p_{1}<p_{2}$ and $B_{2}$ is selected). The control signals $S_{1}$ and $S_{2}$ are dynamically adjusted, so that the MPCC method can immediately respond to the repeated current reference changes between $I_{r e f, 1}$ and $I_{r e f, 2}$. Therefore, the cross regulation can be significantly reduced.

Based on the simulation results, the MPCC method is capable of immediately responding to the current reference variation and of generating the optimal switching control signals. Therefore, the individual charging operation is feasible for charge equalization based on the SI-SIMO DC-DC buck converter with the proposed MPCC method.

\subsubsection{Charge Equalization Performance Based on SI-SIMO DC-DC Buck Converter}

In this section, two test cases are conducted to study the performance of the proposed charge equalization method based on the SI-SIMO DC-DC buck converter. In the first test case, the SOCs of the battery cells are set close to each another. In the second test case, one battery cell is set an initial SOC that is significantly larger than the SOCs of the other battery cells, so that it will be isolated. In these two cases, the number of the battery cells $n$ is set as 3 .

\subsubsection{Test Case 1: Small SOC Difference Condition}

The initial SOCs of three battery cells $S O C_{1}, S O C_{2}$ and $S O C_{3}$ are set as $0.92,0.93$ and 0.94 respectively, which is the small SOC difference condition. The simulation results are shown in Figures 4-10, 4-11 and 4-12. In order to explain the results clearly, every figure is divided into two stages, i.e., $\mathrm{CC}$ stage and $\mathrm{CV}$ stage. Moreover, the CC stage is further divided into three stages as $\mathrm{CC}_{1}$ stage, $\mathrm{CC}_{2}$ stage and $\mathrm{CC}_{3}$ stage.

The $\mathrm{CC}_{1}$ stage is from 0 to $71.7 \mathrm{~s}$. At the beginning of $\mathrm{CC}_{1}$ stage, $S O C_{1}, S O C_{2}$ and $S O C_{3}$ are ranked based on (4-15) and set as $S O C_{1}(1), S O C_{2}(2)$ and $S O C_{3}(3)$ respectively. Thus, $S O C_{\min }$ is equal to $S O C_{1}$. The initial current references are assigned following the algorithm in Figure 4-4(a). Therefore, the current references $i_{R e f, 1}, i_{R e f, 2}$ and $i_{R e f, 3}$ are 


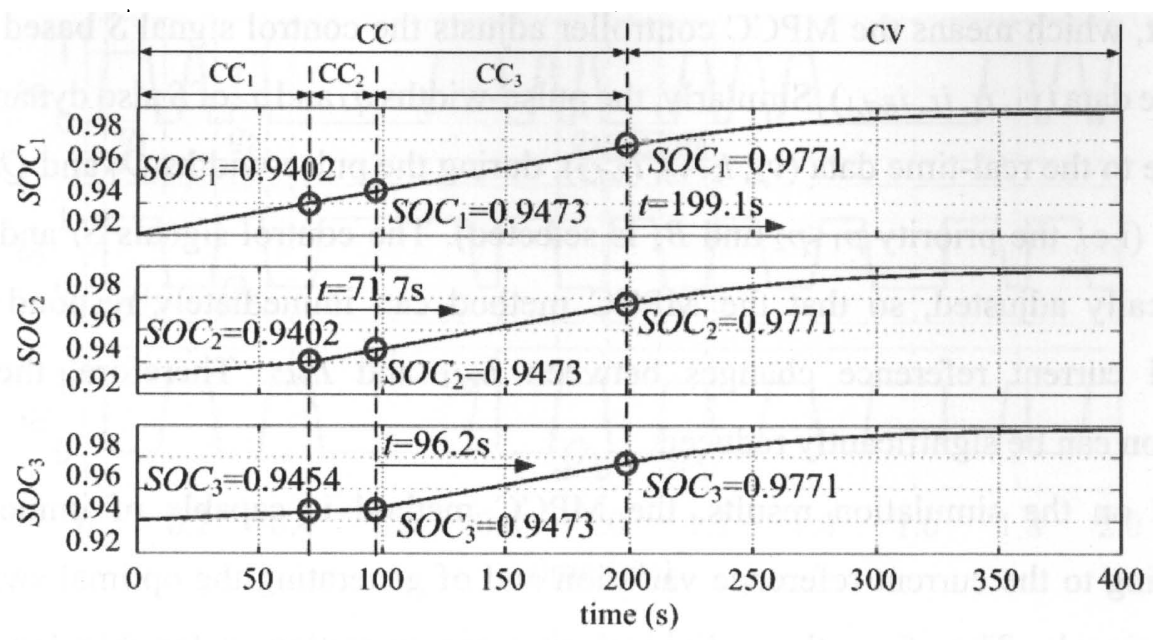

Figure 4-10: SOC curves for small SOC difference condition.

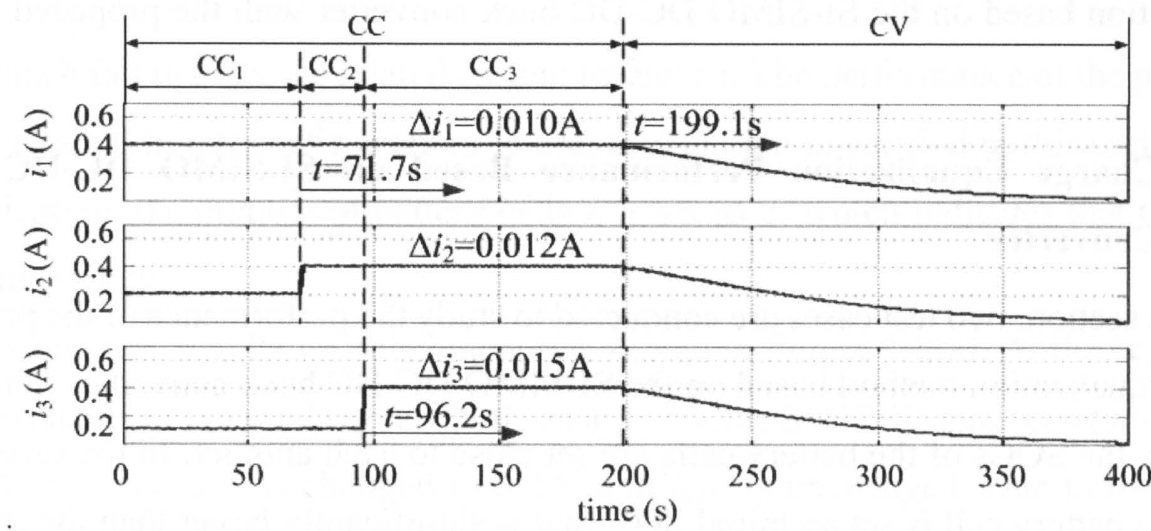

Figure 4-11: Current curves for small SOC difference condition.

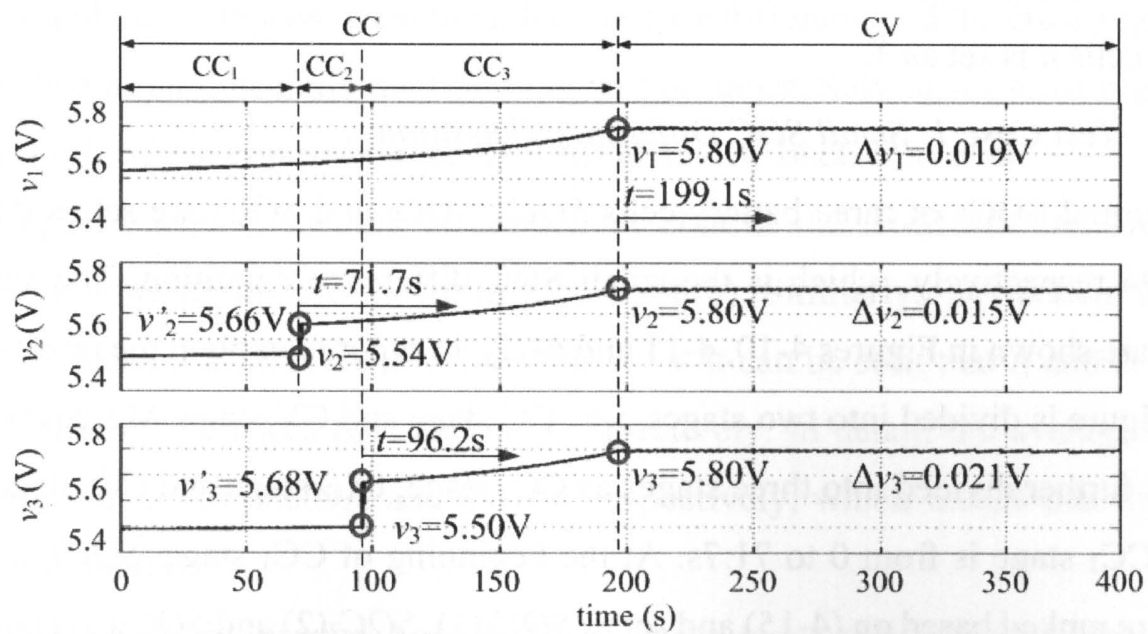

Figure 4-12: Voltage curves for small SOC difference condition.

calculated as $0.4 \mathrm{~A}(1 \mathrm{C}), 0.2 \mathrm{~A}(0.5 \mathrm{C})$ and $0.1 \mathrm{~A}(0.25 \mathrm{C})$ respectively. Figure 4-11 shows that $i_{1}, i_{2}$ and $i_{3}$ are approximately to $0.4 \mathrm{~A}, 0.2 \mathrm{~A}$ and $0.1 \mathrm{~A}$ during $\mathrm{CC}_{1}$ stage. Figure 4- 
10 and Figure 4-12 show that SOCs and voltages are increased gradually during $\mathrm{CC}_{1}$ stage.

The $\mathrm{CC}_{2}$ stage is from $71.7 \mathrm{~s}$ to $96.2 \mathrm{~s}$. After several cycles $T_{C C}, S O C_{1}$ and $S O C_{2}$ are both equal to 0.9402 when $t=71.7 \mathrm{~s}$ as shown in Figure $4-10$. In other words, $S O C_{1}$ catches up with $S O C_{2}$ due to battery cell $B_{1}$ has the fastest charging speed. It can also be seen that $S O C_{3}$ is 0.9454 when $t=71.7 \mathrm{~s}$. Therefore, $S O C_{\min }$ is 0.9402 . Following the current reference re-assignment algorithm in Figure 4-4(b), the current references $i_{R e f, 1}$ and $i_{R e f, 2}$ are re-assigned as $0.4 \mathrm{~A}$, and $i_{R e f, 3}$ remains $0.1 \mathrm{~A}$. Consequently, $i_{1}, i_{2}$ and $i_{3}$ are approximately to $0.4 \mathrm{~A}, 0.4 \mathrm{~A}$ and $0.1 \mathrm{~A}$ during $\mathrm{CC}_{2}$ stage as shown in Figure 4-11. Moreover, the sudden change in $i_{2}$ causes a transient increase of $v_{2}$ from $5.54 \mathrm{~V}$ to $5.66 \mathrm{~V}$ when $t=71.7 \mathrm{~s}$ as shown in Figure 4-12. The $\mathrm{CC}_{3}$ stage is from $96.2 \mathrm{~s}$ to $199.1 \mathrm{~s}$. When $t=96.2 \mathrm{~s}, S O C_{1}, S O C_{2}$ and $S O C_{3}$ are equal to 0.9473 as shown in Figure 4-10, i.e., all battery cells are equally charged. Therefore, all SOCs are equal to $S O C_{\min }$. Consequently, $i_{R e f, 1}, i_{R e f, 2}$ and $i_{R e f, 3}$ are all set as $0.4 \mathrm{~A}$ when $t=96.2 \mathrm{~s}$, following the current references re-assignment algorithm in Figure 4-4(b). As shown in Figure 4-11, $i_{1}$, $i_{2}$ and $i_{3}$ are all regulated approximately to $0.4 \mathrm{~A}$ during $\mathrm{CC}_{3}$ stage. Similarly, $v_{3}$ also has a transient increase from $5.50 \mathrm{~V}$ to $5.68 \mathrm{~V}$ due to the sudden change in $i_{3}$ when $t=96.2 \mathrm{~s}$ as shown in Figure 4-12. $\mathrm{CC}_{3}$ stage is ended when $t=199.1 \mathrm{~s}$, when all voltages reach $V_{\text {nom }}=5.8 \mathrm{~V}$.

During the whole CC stage, the average current ripples $\Delta i_{1}, \Delta i_{2}$ and $\Delta i_{3}$ are about $0.010 \mathrm{~A}, 0.012 \mathrm{~A}$ and $0.015 \mathrm{~A}$ respectively. Therefore, the proposed MPCC method regulates current $i_{1}, i_{2}$ and $i_{3}$ to closely track the varying current references $i_{R e f, 1}, i_{R e f, 2}$ and $i_{R e f, 3}$, and the cross regulation is significantly reduced.

The CV stage is from $199.1 \mathrm{~s}$ to $400 \mathrm{~s}$. When $t=199.1 \mathrm{~s}, v_{1}, v_{2}$ and $v_{3}$ all reach $V_{\text {nom }}=5.8 \mathrm{~V}$ simultaneously as shown in Figure 4-12. Therefore, CV charging starts. Meanwhile, $S O C_{1}, S O C_{2}$, and $S O C_{3}$ are all 0.9771 as shown in Figure 4-10. The current references $i_{R e f, 1}, i_{R e f, 2}$ and $i_{R e f, 3}$ are gradually decreased from $0.4 \mathrm{~A}$ to $0.01 \mathrm{~A}$ with a decrement of $0.01 \mathrm{~A}$ as shown in Figure 4-11, following the current reference assignment algorithm in Figure 4-5(b). During the CV stage, $v_{1}, v_{2}$ and $v_{3}$ are all maintained approximately to $5.8 \mathrm{~V}$, and the average voltage ripples $\Delta v_{1}, \Delta v_{2}$ and $\Delta v_{3}$ are about $0.019 \mathrm{~V}, 0.015 \mathrm{~V}$ and $0.021 \mathrm{~V}$ respectively as shown in Figure $4-12$. CV charging ends when $t=400$ s and $S O C_{1}, S O C_{2}$ and $S O C_{3}$ simultaneously reach 1 as shown in Figure 4-10. 


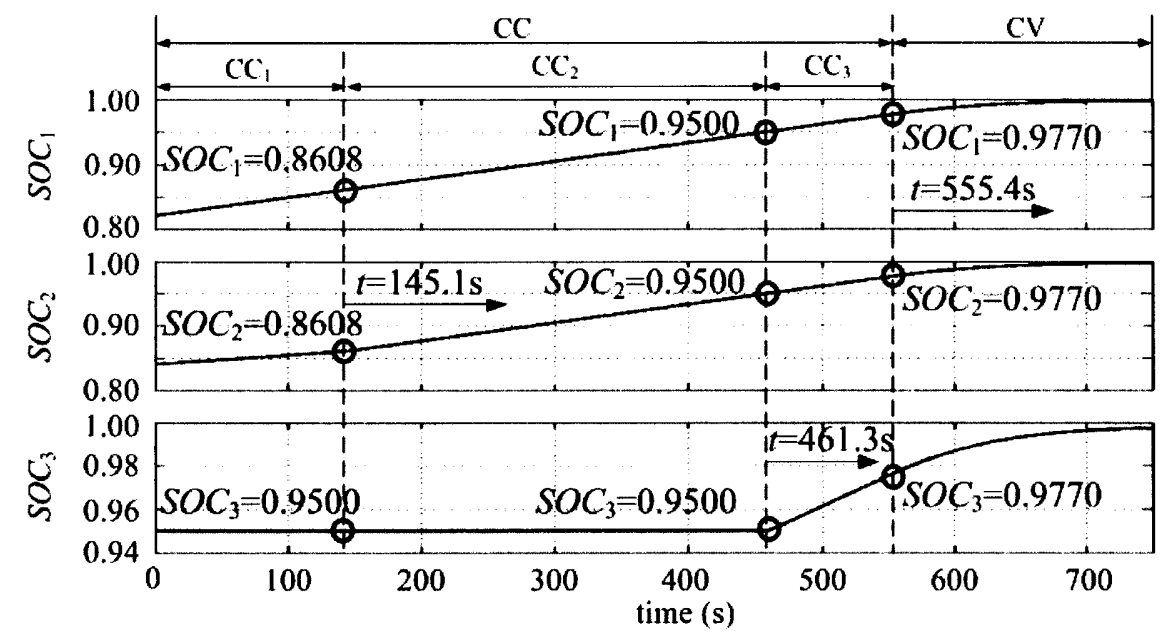

Figure 4-13: SOC curves for large SOC difference condition.

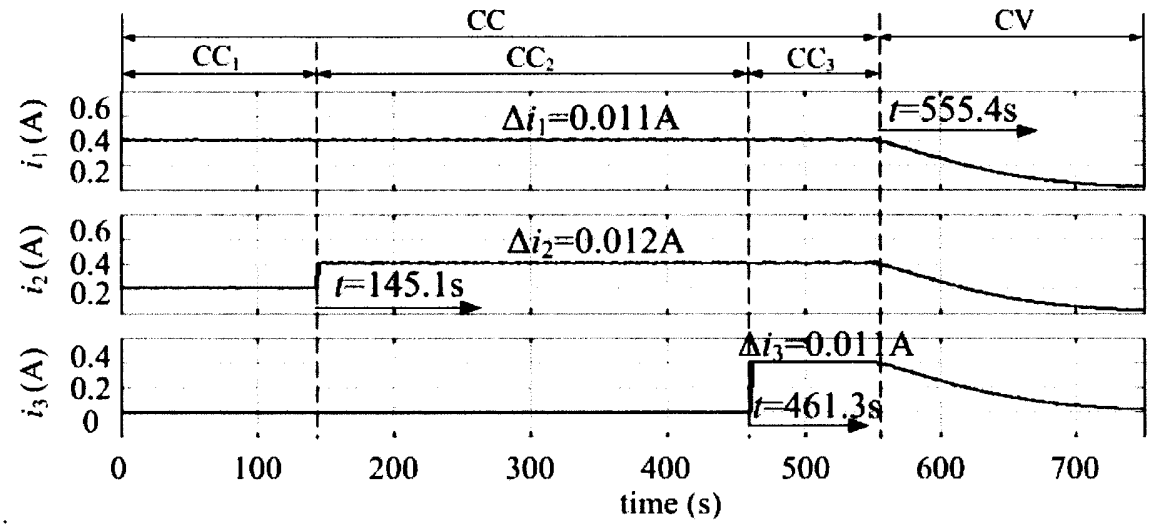

Figure 4-14: Current curves for large SOC difference condition.

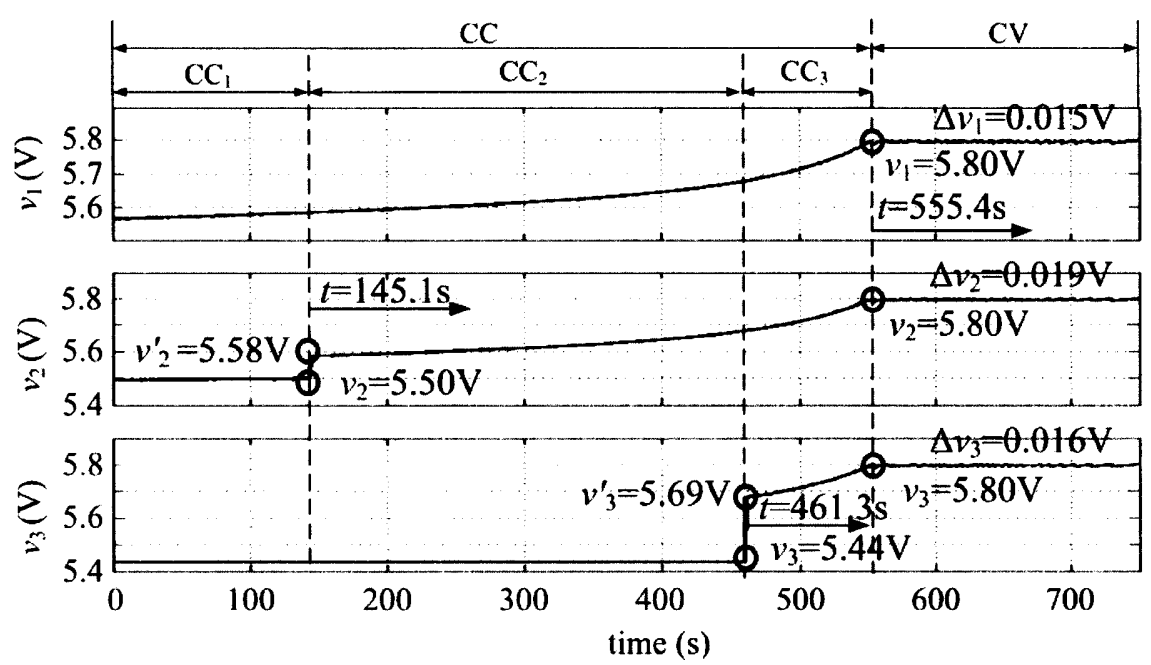

Figure 4-15: Voltage curves for large SOC difference condition.

\subsubsection{Test Case 2: Large SOC Difference Condition}

A more complicated situation is studied in this test case. The initial SOCs are set to $0.82,0.84$ and 0.95 respectively, which is the large SOC difference condition. The 
simulation results are shown in Figure 4-13, 4-14 and 4-15. Similarly, the CC stage (including $C C_{1}, C C_{2}$ and $C C_{3}$ ) and $\mathrm{CV}$ stage are defined.

The $\mathrm{CC}_{1}$ stage is from 0 to $145.1 \mathrm{~s}$. At the beginning of $\mathrm{CC}_{1}$ stage, $S O C_{1}, S O C_{2}$ and $S O C_{3}$ are ranked based on (4-15) and set as $S O C_{1}(1), S O C_{2}(2)$ and $S O C_{3}(3)$ respectively. $S O C_{\min }$ is equal to $S O C_{1}$. The initial current references are assigned following the algorithm in Figure 4-4(a). Therefore, battery cell $B_{3}$ is isolated during the $C C_{1}$ stage because of its large SOC. The current references $i_{R e f, 1,} i_{R e f, 2}$ and $i_{R e f, 3}$ are calculated as $0.4 \mathrm{~A}(1 \mathrm{C}), 0.2 \mathrm{~A}(0.5 \mathrm{C})$ and $0 \mathrm{~A}(0 \mathrm{C})$ respectively. Figure $4-14$ shows that $i_{1}, i_{2}$ and $i_{3}$ are approximately to $0.4 \mathrm{~A}, 0.2 \mathrm{~A}$ and $0 \mathrm{~A}$ during $\mathrm{CC}_{1}$ stage.

The $\mathrm{CC}_{2}$ stage is from $145.1 \mathrm{~s}$ to $461.3 \mathrm{~s}$. After several cycles $T_{C C}, S O C_{l}$ catches up with $S O C_{2}$ and they are both equal to 0.8608 when $t=145.1 \mathrm{~s}$ as shown in Figure 4-13. However, $S O C_{3}$ is still 0.9500 due to battery cell $B_{3}$ is isolated. Therefore, $S O C_{\min }$ is 0.8608. Following the algorithm in Figure 4-4(b), the current references $i_{R e f, 1}$ and $i_{R e f, 2}$ are re-assigned as $0.4 \mathrm{~A}$, and $i_{R e f, 3}$ remains at $0 \mathrm{~A}$. Consequently, $i_{1}, i_{2}$ and $i_{3}$ are approximately to $0.4 \mathrm{~A}, 0.4 \mathrm{~A}$ and $0 \mathrm{~A}$ during $\mathrm{CC}_{2}$ stage as shown in Figure 4-14. Moreover, the sudden change in $i_{2}$ causes a transient increase of $v_{2}$ from $5.50 \mathrm{~V}$ to $5.58 \mathrm{~V}$ when $t=145.1 \mathrm{~s}$ as shown in Figure 4-15.

The $\mathrm{CC}_{3}$ stage is from $461.3 \mathrm{~s}$ to $555.4 \mathrm{~s}$. When $t=461.3 \mathrm{~s}, S O C_{1}, S O C_{2}$ and $S O C_{3}$ are all equal to 0.9500 , as shown in Figure $4-13$, i.e., all battery cells are equally charged. Therefore, all SOCs are equal to $S O C_{m i n}$. Consequently, $i_{R e f, 3}$ is increased to $0.4 \mathrm{~A}$ when $t=461.3 \mathrm{~s}$ following the current references re-assignment algorithm in Figure 4-4(b), i.e., the isolation of battery cell $B_{3}$ is ended. As shown in Figure 4-14, $i_{1}, i_{2}$ and $i_{3}$ are all regulated approximately to $0.4 \mathrm{~A}$ during $\mathrm{CC}_{3}$ stage. Similarly $v_{3}$ also has a transient increase from $5.44 \mathrm{~V}$ to $5.69 \mathrm{~V}$ due to the sudden change in $i_{3}$ when $t=461.3 \mathrm{~s}$, as shown in Figure 4-15. $\mathrm{CC}_{3}$ stage is ended when $t=555.4 \mathrm{~s}$, when all voltages reach $V_{\text {nom }}$. It can be seen from Figure 4-14 that the average current ripples $\Delta i_{1}, \Delta i_{2}$ and $\Delta i_{3}$ are about $0.011 \mathrm{~A}, 0.012 \mathrm{~A}$ and $0.011 \mathrm{~A}$ respectively, during the whole $\mathrm{CC}$ stage.

The CV stage is from $555.4 \mathrm{~s}$ to $755.0 \mathrm{~s}$. When $t=555.4 \mathrm{~s}, v_{1}, v_{2}$ and $v_{3}$ reach $V_{\text {nom }}=5.8 \mathrm{~V}$ simultaneously as shown in Figure 4-15. Meanwhile, $S O C_{1}, S O C_{2}$ and $S O C_{3}$ are all 0.9770 as shown in Figure 4-13. Similarly, the current references $i_{R e f, 1}, i_{R e f, 2}$ and $i_{R e f, 3}$ are gradually decreased from $0.4 \mathrm{~A}$ to $0.01 \mathrm{~A}$ with a decrement of $0.01 \mathrm{~A}$ as shown in Figure 4-14. $v_{1}, v_{2}$, and $v_{3}$ are all maintained at approximately 5.8V. During the CV stage, the average voltage ripples $\Delta v_{1}, \Delta v_{2}$ and $\Delta v_{3}$ are about $0.015 \mathrm{~V}, 0.019 \mathrm{~V}$ and 
Table 4-2 Comparison of different charging equalization methods for " $n$ " series-connected battery cells

\begin{tabular}{c|c|c|c|c}
\hline \hline & $\begin{array}{c}\text { Cuk Converter } \\
{[101]}\end{array}$ & $\begin{array}{c}\text { Single-Output Converter } \\
{[105]}\end{array}$ & $\begin{array}{c}\text { Converter-Multiple- } \\
\text { Output-Transformer } \\
{[107]}\end{array}$ & SIMO \\
\hline Inductor & $2 \mathrm{n}-2$ & $\mathrm{n}$ & 0 & 1 \\
\hline Diode & 0 & $\mathrm{n}$ & 1 & 1 \\
\hline Switch & $2 \mathrm{n}+2$ & $2 \mathrm{n}+1$ & $2 \mathrm{n}+1$ & $2 \mathrm{n}+1$ \\
\hline Transformer & 0 & 0 & 1 or 2 & 0 \\
\hline
\end{tabular}

$0.016 \mathrm{~V}$ respectively, as shown in Figure $4-15 \mathrm{CV}$ charging ends when $t=755.0 \mathrm{~s}$ and $S O C_{1}, S O C_{2}$ and $S O C_{3}$ simultaneously reach 1 as shown in Figure 4-13.

\subsubsection{Comparison with Existing Methods for Charging Equalization}

The number of components required by the proposed charge equalization system is compared to other individual charge equalization systems as shown in Table 4-2. It can be seen that the proposed system requires less components to realize the balance charging of the series-connected batteries. In particular, the proposed method only adopts single inductor, which significantly reduces the circuit size and relieves EMI.

\subsection{Conclusion}

In this chapter, a battery charge equalization method has been proposed for seriesconnected battery cells in EVs. This method uses the SI-SIMO DC-DC converter to realize the individual charging operation for each battery cell. In order to control the SI-SIMO DC-DC converter, the MPCC method has been developed to solve the cross regulation problem. Moreover, relevant current reference assignment algorithms for CC and $\mathrm{CV}$ charging have also been proposed, which provide proper references for each battery cell.

Several simulation studies have been conducted to verify the performance of the proposed charge equalization method including MPCC method and current reference assignment algorithms for CC and CV charging. First, the proposed the MPCC method achieves the $\mathrm{CC}$ charging and $\mathrm{CV}$ charging with the ripples within tolerable range. Second, the proposed MPCC method is able to solve the cross regulation problem of the SI-SIMO DC-DC converter, i.e., the output currents track the assigned current references closely. This capability establishes the basis for individual charging 
CHAPTER 4 MODEL PREDICTIVE CURRENT CONTROL FOR CHARGING EQUALIZATION BASED ON SI-SIMO DC-DC CONVERTER

operations. Finally, the proposed charge equalization method is verified under the conditions of small and large SOC differences. The simulation results have demonstrated that the proposed method can achieve the charge equalization in different conditions. 


\section{CHAPTER 5 POWER SHARING AND CROSS REGULATION SUPPRESSION METHOD FOR SI-MIMO DC-DC CONVERTER}

In this chapter, the MPC method is applied to the SI-MIMO DC-DC converter, with the power sharing method and time-multiplexing method. Therefore, a power sharing and cross regulation method is developed.

\subsection{Operational Principles}

\subsubsection{SI-MIMO DC-DC Buck Converter}

A SI-MIMO buck converter is proposed for study as shown in Figure 5-1. On the input side, there are $m$ input sources $v_{i n, 1}, v_{i n, 2}, \ldots, v_{i n, i}, \ldots v_{i n, m}$ with corresponding switches $S_{i n, 1}, S_{i n, 2}, \ldots, S_{i n, i}, \ldots, S_{i n, m}$, and the internal resistances and input currents for each source are $R_{i n, 1}, R_{i n, 2}, \ldots, R_{i n, i}, \ldots, R_{i n, m}$ and $i_{i n, 1}, i_{i n, 2}, \ldots, i_{i n, i}, \ldots, i_{i n, m}$ respectively $D$ denotes the freewheeling diode. The inductor is $L$ with an ESR $R_{L}$, and the current through the inductor $L$ is $i_{L}$. On the output side, the selection switches are $S_{\text {out }, 1}, S_{\text {out }, 2}, \ldots$, $S_{\text {out }, j}, \ldots, S_{\text {out }, n}$. For the $j$ th load $R_{j}$, the corresponding voltage, current and capacitor are $v_{o u t, j}, i_{\text {out } j}$ and $C_{j}$ respectively.

For the proposed SI-MIMO buck converter, the most important operational principle is that only one power source and one load are connected to the inductor $L$ at a given instant. Therefore, a SISO buck converter is formed. For example, the $i$ th power source and the $j$ th load are selected, and the detailed operation is shown in Figure 5-2. The proposed power sharing and cross regulation suppression method is to determine the control signals $q_{1}, q_{2}, \ldots, q_{i}, \ldots, q_{m}$ for switches $S_{i n, 1}, S_{i n, 2}, \ldots, S_{i n, i}, \ldots, S_{i n, m}$, and $q_{1}^{\prime}$, $q_{2}, \ldots, q_{j}, \ldots, q_{n}$ ' for switches $S_{o u t, 1}, S_{o u t, 2}, \ldots, S_{\text {out }, j}, \ldots, S_{\text {out }, n}$ based on power sharing of input source and the load demand of output side simultaneously, and following the operational principle shown in Figure 5-2.

\subsubsection{MPC for SI-MIMO Buck Converter}

Assuming the $i$ th input source $v_{i n, i}$ and the $j$ th load $R_{j}$ are selected to form a SISO buck converter shown in Figure 5-2, the MPC method is applied to this temporary SISO buck converter for output voltage $v_{o u t, j}$ regulation. 


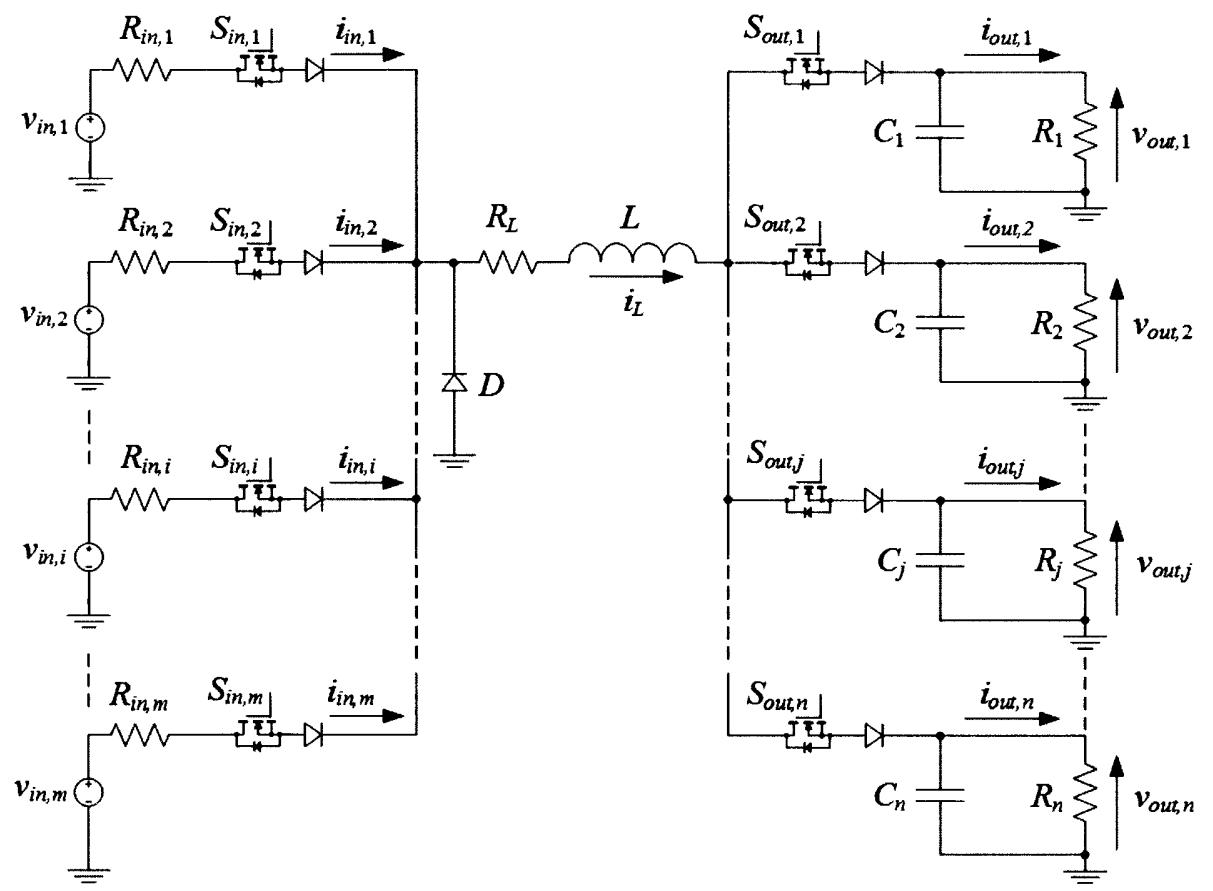

Figure 5-1: Architecture of the SI-MIMO buck converter.

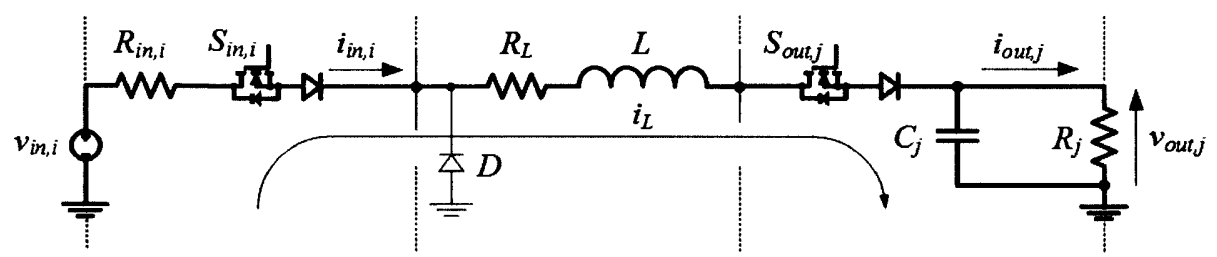

(a)

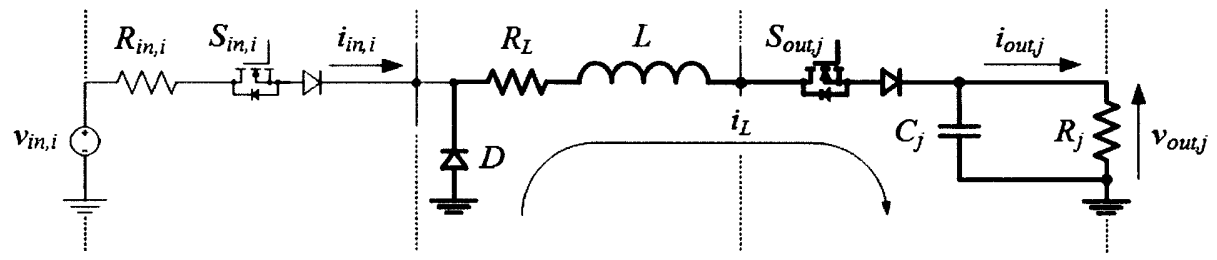

(b)

Figure 5-2: Operation of the SI-MIMO buck converter. (a) $S_{i n, i}$ and $S_{\text {out }, j}$ are on, the other switches are off. (b) $S_{\text {out }, j}$ is on, the other switches are off.

\subsubsection{State-space Model}

In order to implement the MPC method, a state-space model is built. The characteristic differential equations of inductor $L$ and capacitor $C_{j}$ of the $j_{t h}$ load $R_{j}$ are

$$
\begin{gathered}
C_{j} \frac{d v_{\text {out }, j}}{d t}=i_{L}-i_{\text {out }, j} \\
L \frac{d i_{L}}{d t}=q_{M P C} v_{i n, i}-v_{o u t, j}-i_{L} R_{L}
\end{gathered}
$$


where $q_{M P C}$ is the control signal generated by the MPC method to control the switch $S_{i n, i}$ in the SISO buck converter, and $R_{i n, i}$ is neglected since its value is small.

Because the MPC method is operated in discrete time with a preset time step $t_{s}$, the discrete formats of the continuous equations (5.1) and (5.2) are needed. For (5.1), the discretization format when $t$ is equal to $k$ is expressed as

$$
v_{o u t, j}(k+1)-v_{o u t, j}(k)=\frac{t_{s}}{C_{j}}\left(i_{L}(k)-\frac{v_{o u t, j}(k)}{R_{j}}\right)
$$

Therefore, when $t$ is equal to $k-1,(5.3)$ can be re-written as

$$
v_{\text {ou }, j}(k)-v_{\text {out }, j}(k-1)=\frac{t_{s}}{C_{j}}\left(i_{L}(k-1)-\frac{v_{\text {out }, j}(k-1)}{R_{j}}\right)
$$

The left side of (5.4) is defined as $\Delta v_{o u t, j}(k)$. Similarly, $\Delta i_{L}(k)$ and $\Delta q_{M P C}(k)$ can be defined. Using (5.3) to subtract (5.4), it can be obtained as

$$
\Delta v_{\text {out }, j}(k+1)=\Delta v_{\text {out }, j}(k)+\frac{t_{s}}{C_{j}} \Delta i_{L}(k)-\frac{t_{s}}{R_{j} C_{j}} \Delta v_{o u t, j}(k)
$$

Similarly, the discrete formats of (5.2) can be obtained as

$$
\Delta i_{L}(k+1)=\left(1-\frac{t_{s} R_{L}}{L}\right) \Delta i_{L}(k)-\frac{t_{s}}{L} \Delta v_{o u t, j}(k)+\frac{t_{s} v_{i n, i}}{L} \Delta q_{M P C}(k)
$$

Moreover, the relationship of $v_{\text {out }, j}(k+1)$ and $v_{\text {out }, j}(k)$ can be expressed as follows:

$$
v_{\text {out }, j}(k+1)=v_{\text {out }, j}(k)+\Delta v_{\text {out }, j}(k+1)
$$

By substituting (5.5) into (5.7), the expression of $v_{o u t, j}(k+1)$ can be re-written as follows:

$$
v_{\text {out }, j}(k+1)=\frac{t_{s}}{C_{j}} \Delta i_{L}(k)+\left(1-\frac{t_{s}}{R_{j} C_{j}}\right) \Delta v_{o u t, j}(k)+v_{\text {out }, j}(k)
$$

With (5.5), (5.6) and (5.8), the augmented discrete-time state-space model is obtained as follows:

$$
\begin{aligned}
& x_{j}(k+1)=\bar{A}_{j} x_{j}(k)+\bar{B}_{j} \Delta q_{M P C}(k) \\
& y_{j}(k)=\bar{C}_{j} x_{j}(k)
\end{aligned}
$$

$x_{j}(k)=\left[\Delta i_{L}(k) \Delta v_{o u t, j}(k) y_{j}(k)\right]^{T}$ is the state vector of the SISO buck converter, and the output voltage of the SISO buck converter is $y_{j}(k)=v_{\text {out }}(k)$. The coefficient matrices are

$$
\bar{A}_{j}=\left[\begin{array}{ccc}
1-t_{s} R_{L} / L & -t_{s} / L & 0 \\
t_{s} / C_{j} & 1-t_{s} / R_{j} C_{j} & 0 \\
t_{s} / C_{j} & 1-t_{s} / R_{j} C_{j} & 1
\end{array}\right]
$$




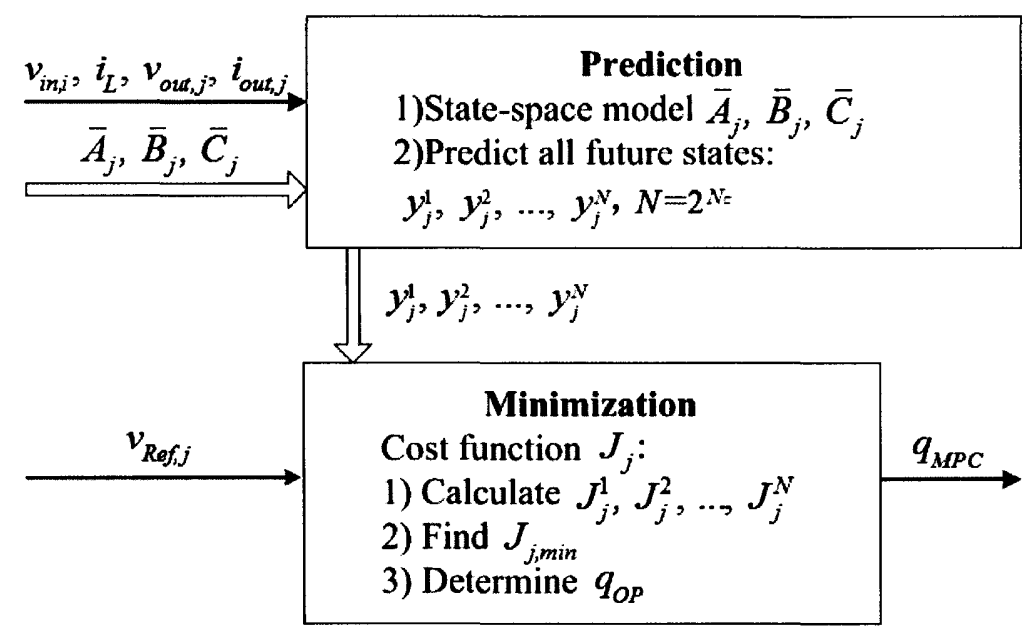

Figure 5-3: Block diagram of MPC method for SISO buck converter.

$$
\begin{gathered}
\bar{B}_{j}=\left[\begin{array}{lll}
t_{s} v_{i n, i} / L & 0 & 0
\end{array}\right]^{T} \\
\bar{C}_{j}=\left[\begin{array}{lll}
0 & 0 & 1
\end{array}\right]
\end{gathered}
$$

In the MPC theory, a cost function is imperative to determine the optimal control action for output regulation [112]. There are three parameters predict horizon $N_{P}$, control horizon $N_{C}$ and Lagrange multiplier $\lambda$ in the cost function. Moreover, the control horizon $N_{C}$ is the length of control action combinations so that the number of all possible control action combinations is $2^{N c}$. The cost function $J_{j}$ for the $j$ th load $R_{j}$ is defined as follows:

$$
J_{j}=\sum_{l=1}^{N_{P}}\left(y_{j}(k+l)-v_{R e f, j}(k+l)\right)^{2}+\lambda \sum_{l=1}^{N_{C}}\left(\Delta q_{M P C}(k+l-1)\right)^{2}
$$

where $v_{R e f, j}$ is the $j$ th voltage reference for $v_{o u t, j}$.

Based on the previous discussion, the block diagram of MPC method is shown in Figure 5-3. In real-time operation, the data of the selected $i$ th power source and $j$ th load, i.e., $v_{i n, i}, v_{o u t, j}, i_{o u t, j}$, the inductor current $i_{L}$ and the corresponding coefficient matrices $\bar{A}_{j}, \bar{B}_{j}, \bar{C}_{j}$ are sent to the prediction block. With the state-space model, all possible future states $\left(y_{j}^{1}, y_{j}^{2}, \ldots, y_{j}^{N}\right)$ can be predicted, where $N$ is equal to $2^{N c}$. This is because that the number of all possible control action combinations is $2^{N c}$. Then, $\left(y_{j}^{1}, y_{j}^{2}, \ldots, y_{j}^{N}\right)$ and the corresponding voltage reference $v_{R e f, j}$ are sent to the minimization block and substituted into the cost function $J_{j}$ to get a series values of $\left(J_{j}^{1}, J_{j}^{2}, \ldots, J_{j}^{N}\right)$. The control action combination, which can minimize the cost function $J_{j}$ as $J_{j, m i n}$, is the optimal one. The 
first control action $q_{O P}$ in the optimal combination is applied into the targeted SISO buck converter as $q_{M P C}$ for $v_{o u t, j}$ regulation.

\subsubsection{Constraints of Inductor Current and Control Signals}

Compared to the conventional methods, the MPC method is able to incorporate constraints according to practical condition, which contributes to a better regulation performance. There are two main constraints: constraints for inductor current $i_{L}(k)$ and constraints for control signal $q_{M P C}(k)$.

Generally, the practical inductor has a current rating $I_{c r}$, which determines the tolerable maximum current through the inductor. Therefore, an upper bound for $i_{L}(k)$ is necessary. Moreover, this upper bound brings an additional benefit, which is relieving or even avoiding the overshoot. Another important consideration is to guarantee that the SIMO buck converter works in the CCM, which also relieves the peak current of the inductor. Hence, a lower bound for $i_{L}(k)$ is also required. Therefore, the constraints for $i_{L}(k)$ are expressed as follows:

$$
\alpha I_{c r} \leq i_{L}(k) \leq \beta I_{c r}
$$

where the adjustable coefficients $\alpha$ and $\beta$ fulfill the relationship $0<\alpha<\beta<1$. By properly setting the values of $\alpha$ and $\beta$, the current of inductor $i_{L}(k)$ is regulated in a suitable range.

The control signal constraints are expressed as follows:

$$
\begin{aligned}
\Delta q_{M P C}(k) & \in\{-1,0,1\} \\
q_{M P C}(k) & \in\{0,1\}
\end{aligned}
$$

These constraints guarantee that the generated optimal control signal is practical and can be applied to the switches directly.

\subsubsection{Power Sharing Method}

\subsubsection{Duty Cycle Calculation}

The power sharing method helps to determine the input powers of the multiple-input sources. The input power $P_{i n, i}$ from the $i$ th source can be expressed as follows:

$$
P_{i n, i}=v_{i n, i} \bar{i}_{i n, i}
$$




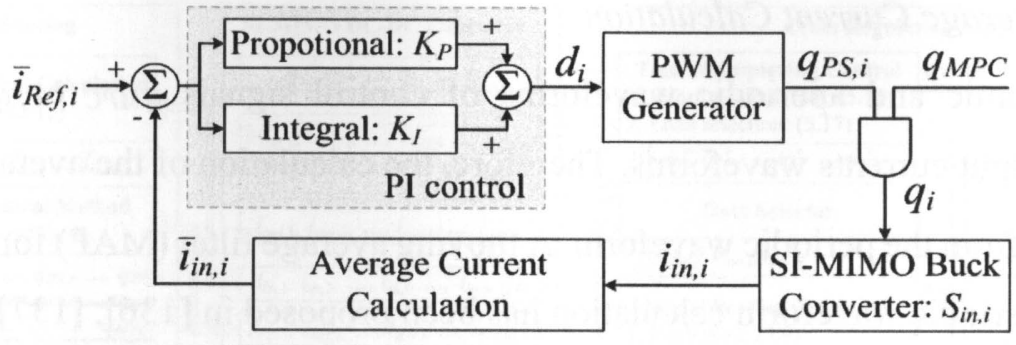

(a)

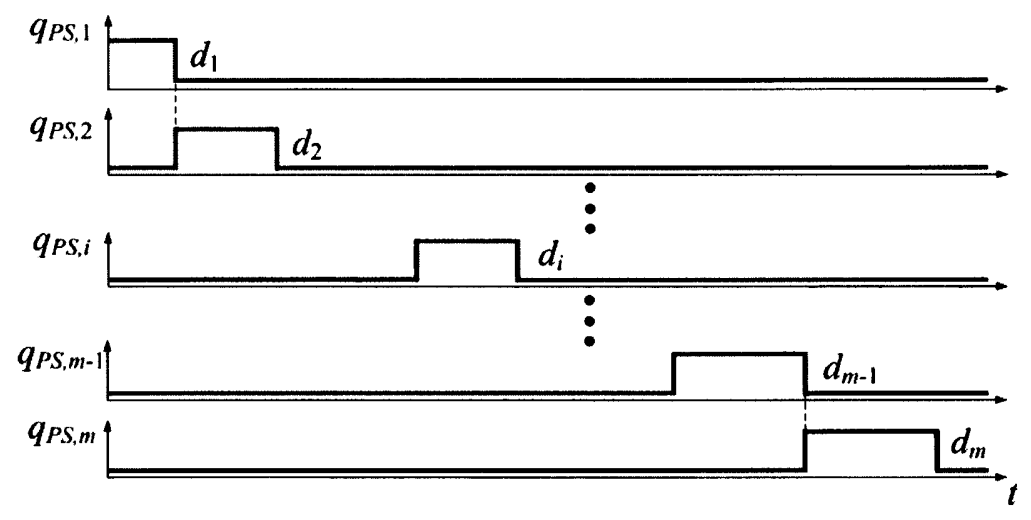

(b)

Figure 5-4: Illustration of power sharing control. (a) Block diagram of PI-based method. (b) Waveforms of control signals $q_{P S, 1}, q_{P S, 2}, \ldots, q_{P S, i}, \ldots, q_{P S, m}$.

where $\bar{i}_{i n, i}$ is the average value of the $i$ th input current $i_{i n, i}$. Because $v_{i n, i}$ is a constant value, $P_{i n, i}$ is determined by the value of $\bar{i}_{i, i}$. Hence, controlling $\bar{i}_{i n, i}$ is equivalent to controlling $P_{i n, i}$ because the input voltage is regarded as constant. A block diagram for $\bar{i}_{i, i}\left(P_{i n, i}\right)$ control is shown in Figure 5-4(a), where $\bar{i}_{\text {Ref }, i}$ is the reference for $\bar{i}_{i, i}$. It is a PI-based method with the proportional and integral terms $K_{P}$ and $K_{I}$, and it is able to generate duty cycle $d_{i}$ for the $i$ th control signal $q_{P S, i}$. Furthermore, $q_{i}$ is the logical conjunction of $q_{P S, i}$ and $q_{M P C}$ and applied into the corresponding switch $S_{i n, i}$ in the SI-MIMO buck converter. Moreover, an algorithm for average current calculation is important to achieve $\bar{i}_{i, i}$, and it will be discussed in the following subsection.

In Figure 5-4(b), the waveforms of $q_{P S, 1}, q_{P S, 2}, \ldots, q_{P S, i}, \ldots, q_{P S, m}$ are presented. With the previous PI-based method, all duty cycles $d_{1}, d_{2}, \ldots, d_{i}, \ldots, d_{m}$ can be determined. Specially, the $m$ th duty cycle $d_{m}$ is calculated based on $d_{1}, d_{2}, \ldots, d_{i}, \ldots, d_{m-1}$ and expressed as follows:

$$
d_{m}=1-\sum_{i=1}^{m-1} d_{i}
$$




\subsubsection{Average Current Calculation}

The dynamic and aperiodic waveforms of control signals $q_{M P C}$ or $q_{P S, i}$ lead to aperiodic input currents waveforms. Therefore, the calculation of the average value $\bar{i}_{i, i}$ is different from the periodic waveform. A moving average filter (MAF) for the average value of aperiodic waveform calculation has been proposed in [136], [137]. Moreover, the MAF can be regarded as a low-pass filter. The MAF operation is expressed as follows:

$$
\bar{i}_{i n, i}=\int_{t}^{t+T w} i_{i n, i}(t) d t
$$

where $T_{w}$ is the time window. As discussed in [137], $T_{w}$ can be selected as the integer time of the preset time step $t_{s}$. Moreover, there is an intrinsic delay of MAF because it takes time to collect enough data of $i_{i n, i}$ for average value calculation, which means $\bar{i}_{i n, i}$ cannot reflect the transient variation of $i_{i n, i}$. This phenomenon will be observed in simulation and experimental results.

\subsubsection{Time-Multiplexing Method}

The selection signals $q_{1}^{\prime}, q_{2}{ }^{\prime}, \ldots, q_{j}^{\prime}, \ldots, q_{n}^{\prime}$ are used to control the switches $S_{\text {out }, 1}$, $S_{\text {out }, 2}, \ldots, S_{\text {out } j}, \ldots, S_{\text {out }, n}$ to select the loads. As mentioned previously, the timemultiplexing method is adopted to determine the values of $q_{1}^{\prime}, q_{2}^{\prime}, \ldots, q_{j}^{\prime}, \ldots, q_{n}^{\prime}$. In order to perform the time-multiplexing method, a priority calculation algorithm is developed as follows:

$$
p_{i}>p_{j}, \text { if }\left(v_{\text {out }, i}-v_{R e f, i}\right) \leq\left(v_{o u t, j}-v_{R e f, j}\right)
$$

where $p_{i}$ and $p_{j}$, and $v_{R e f, i}$ and $v_{R e f, j}$ are the priority and voltage references for the $i$ th and $j$ th load $R_{i}$ and $R_{j}$ respectively.

To determine the load with highest priority, the $p_{\max }$ is defined as the maximum value among all priorities. Consequently, the selection control signal $q_{j}$ ' can be determined based on the corresponding priority $p_{j}$ as shown below.

$$
\left\{\begin{array}{l}
q_{j}=1, \text { if } p_{j}=p_{\max } \\
q_{j}=0, \text { if } p_{j}<p_{\max }
\end{array}\right.
$$

After the calculation of all priorities, the values of $q_{1}^{\prime}, q_{2}^{\prime}, \ldots, q_{j}^{\prime}, \ldots, q_{n}^{\prime}$ are set. Assuming that the $j$ th load is selected to be supplied in the following control cycle, it will form the SISO buck converter as shown in Figure 5-2. 


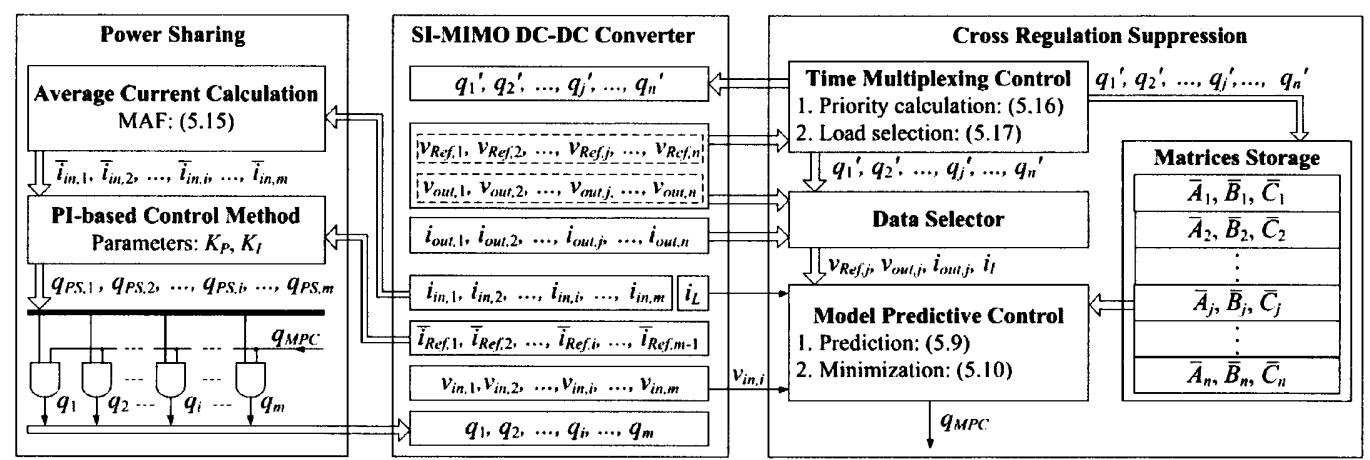

Figure 5-5: Overview of the proposed power sharing and cross regulation suppression method.

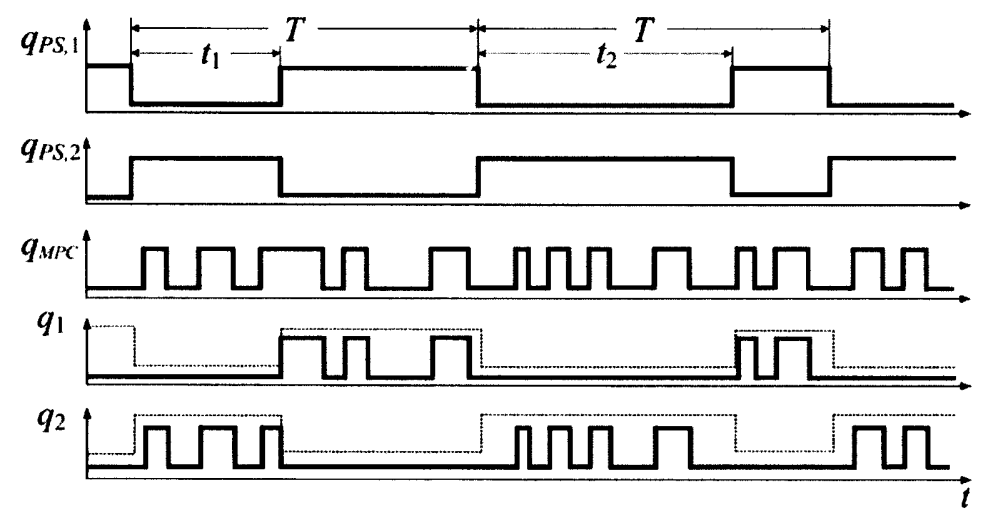

Figure 5-6: Power sharing control signal waveforms based on SI-DIDO buck converter.

\subsection{Controller ANd COMPonent Design}

The designs for power sharing controller and cross regulation suppression controller are discussed in this section, and the overview of the proposed method is shown in Figure 5-5.

\subsubsection{Power Sharing Controller}

\subsubsection{Control Scheme}

The power sharing controller is shown on the left side of Figure 5-5. It can be seen that the input currents $i_{i n, 1}, i_{i n, 2}, \ldots, i_{i n, i}, \ldots, i_{i n, m}$ are sent to the average current calculation block. The average currents $\bar{i}_{i n, 1}, \bar{i}_{i n, 2}, \ldots, \bar{i}_{i n, i} \ldots, \bar{i}_{i n, m}$ can be obtained using the MAF with a suitable long time window $T_{w}$. Consequently, the control signals $q_{P S, 1}$, $q_{P S, 2}, \ldots, q_{P S, i}, \ldots, q_{P S, m}$ are generated based on the average currents and corresponding current references. As discussed previously, the control signal $q_{i}$ for $S_{i n, i}$ is the logical conjunction of $q_{P S, i}$ and $q_{M P C}$. Therefore, $q_{1}, q_{2}, \ldots, q_{i}, \ldots, q_{m}$ are expressed as follows:

$$
q_{i}=q_{P S i} \cap \quad 1,2, \ldots, m
$$




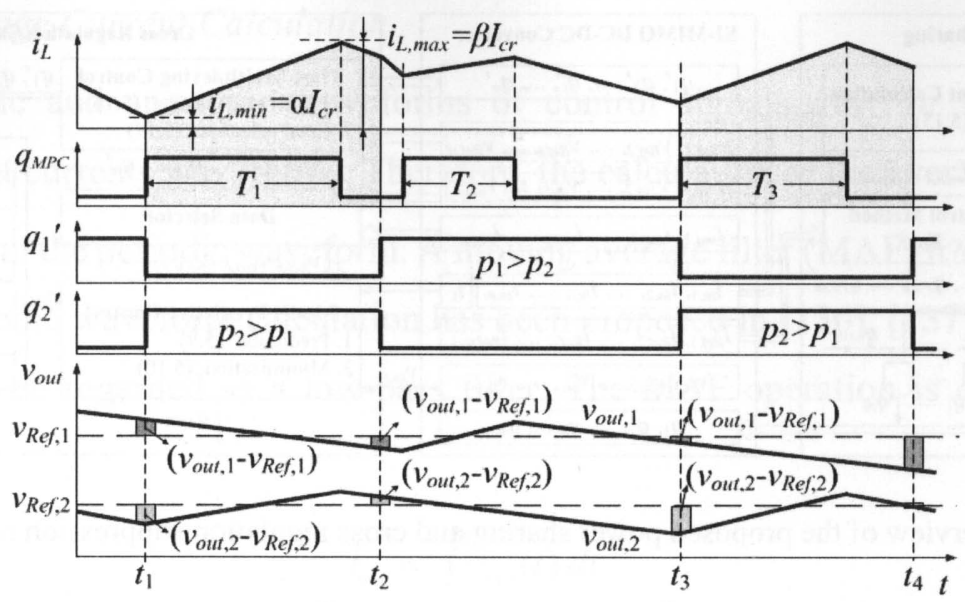

Figure 5-7: Cross regulation suppression control signal waveforms based on SI-DIDO buck converter.

\subsubsection{Control Signal Waveforms}

To explain the control signal waveforms of power sharing controller, the SI-DIDO buck converter is taken as the example for illustration. In Figure 5-6, it can be seen that $q_{1}$ or $q_{2}$ are the logical conjunction of $q_{P S, 1} \cap q_{M P C}$ or $q_{P S, 2} \cap q_{M P C}$. Moreover, $q_{M P C}$ is generated by the MPC method so that $q_{1}$ and $q_{2}$ have varying switching frequencies and dynamic duty cycles, which will influence the power sharing effect. Therefore, the duty cycles of $q_{P S, 1}$ and $q_{P S, 2}$ are also dynamically adjusted to maintain the power sharing ratios of two power sources. For example, the two adjacent duty cycle of $q_{P S, 1}$ are $t_{1} / T$ and $t_{2} / T$ respectively.

\subsubsection{Cross Regulation Suppression Controller}

After the $i$ th power source is determined by the power sharing method, the cross regulation suppression method based on the MPC method and time-multiplexing method will further generate $q_{M P C}$ and $q_{1}{ }^{\prime}, q_{2}, \ldots, q_{j}, \ldots, q_{n}{ }^{\prime}$ to regulate the output voltages individually so that the cross regulation is suppressed.

\subsubsection{Control Scheme}

The cross regulation suppression controller is shown in the right side of Figure 5-5. Firstly, the real-time output voltages $v_{o u t, 1}, v_{\text {out }, 2}, \ldots, v_{\text {out }, j}, \ldots, v_{\text {out }, n}$ and corresponding voltage references $v_{\text {Ref, },}, v_{\text {Ref, },}, \ldots, v_{\text {Ref }, j}, \ldots, v_{\text {Ref, } n}$ will be sent to the time-multiplexing block to perform the priority calculation. Then, all load selection signals $q_{1}, q_{2}, \ldots$, $q_{j}^{\prime}, \ldots, q_{n}$ ' are obtained based on all priorities $p_{1}, p_{2}, \ldots, p_{j}, \ldots, p_{n}$. The selection signals are applied to control the output switches $S_{\text {out }, 1}, S_{\text {out }, 2}, \ldots, S_{\text {out }, j}, \ldots, S_{\text {out }, n}$ directly. 
Meanwhile, the selection signals help to determine the data $\left(v_{R e f, j}, v_{o u t, j}, i_{\text {out } j}\right)$ of $j$ th load and corresponding coefficient matrices $\bar{A}_{j}, \bar{B}_{j}, \bar{C}_{l}$ for prediction. The prediction also needs the information of inductor current $i_{l}$ and selected input voltage $v_{i n, i}$. Finally, the control signal $q_{M P C}$ can be generated to regulate the output voltage $v_{o u t, j}$.

\subsubsection{Control Signal Waveforms}

Similarly, the SI-DIDO buck converter is used to explain the control signal waveforms of the cross regulation suppression controller, as shown in Figure 5-7. It can be seen that the priority calculation happens when $t=t_{1}, t_{2}$ and $t_{3}$. When $t=t_{1}$ and $t_{3}$, the value of $\left(v_{o u t, 1}-v_{R e f, 1}\right)$ is greater than the value of $\left(v_{o u t, 2}-v_{R e f, 2}\right)$. Hence, in the time slots $\left(t_{1} \sim t_{2}\right)$ and $\left(t_{3} \sim t_{4}\right), R_{1}$ has lower priority $p_{1}$ than $p_{2}$ of $R_{2}$, so that $q_{1}^{\prime}=0$ and $q_{2}^{\prime}=1$. On the contrary, $p_{1}$ is greater than $p_{2}$ in the time slot $\left(t_{2} \sim t_{3}\right)$ because the value of $\left(v_{\text {out }, 1}-v_{R e f, 1}\right)$ is less than the value of $\left(v_{o u t, 2}-v_{R e f, 2}\right)$, therefore, $q_{1}{ }^{\prime}=1$ and $q_{2}{ }^{\prime}=0$. The lengths of $\left(t_{1} \sim t_{2}\right)$, $\left(t_{2} \sim t_{3}\right)$ and $\left(t_{3} \sim t_{4}\right)$ are determined by the time-multiplexing method. Additionally, the duty cycles of $q_{M P C}$ are $T_{1} /\left(t_{2}-t_{1}\right), T_{2} /\left(t_{3}-t_{2}\right)$ and $T_{3} /\left(t_{4}-t_{3}\right)$ respectively, and the values of these duty cycles are determined dynamically by the MPC method based on real-time data $\left(v_{R e f, j}, v_{o u t, j}, i_{o u t}, j, i_{L}, v_{\text {in, }, i}\right)$ conditions. As a result, the output voltages $v_{o u t, 1}$ and $v_{o u t, 2}$ regulated close to the corresponding references by the MPC method and timemultiplexing method. In other words, the cross regulation is suppressed.

It also can be seen that the inductor current $i_{L}$ has a minimum value $\alpha I_{c r}$ and a maximum value $\beta I_{c r}$ because of the preset constraints, which guarantee CCM operation and avoid high peak current through $L$. Moreover, $i_{L}$ is in a pseudo steady state resulting from the dynamic duty cycles of $q_{M P C}$.

\subsubsection{Component Design Consideration}

Generally, the selection of the inductor and capacitors for buck converter design is following the equations from [134] as shown below.

$$
\begin{aligned}
& L=\left(v_{\text {in }, i}-v_{\text {out }, j}\right) \frac{v_{\text {out }, j}}{v_{\text {in }, i} f_{\text {sw }} \Delta i_{L}} \\
& C_{j}=\frac{L\left(i_{\text {out }, j}+0.5 \Delta i_{L}\right)^{2}}{\left(v_{\text {out }, j}+\Delta v_{\text {out }, j}\right)^{2}-v_{\text {out }, j}{ }^{2}}
\end{aligned}
$$

where $f_{s w}$ is the switching frequency of $q_{M P C}, \Delta i_{L}$ is the ripple of inductor current $i_{l}$ and $\Delta v_{\text {out }, j}$ is the output voltage ripple. It can be seen that switching frequency $f_{s w}$ plays a 
CHAPTER 5 POWER SHARGING AND CROSS REGULATION SUPPRESSION METHOD FOR SINGLE-INDUCTOR MULITPLE-INPUT MULTIPLE-OUTPUT DC-DC CONVERTER

Table 5-1 Simulation parameters of the SI-DIDO buck converter

\begin{tabular}{c|c|c|c|c|c}
\hline \hline Symbol & $v_{\text {in, } i}$ & $v_{\text {out } j}$ & $R_{i}$ & $t_{s}$ & $f_{s w}$ \\
\hline Value & $20,24,30 \mathrm{~V}$ & $8,10,12 \mathrm{~V}$ & $8 \sim 10 \Omega$ & $5 \mu \mathrm{s}$ & $20 \sim 100 \mathrm{kHz}$ \\
\hline Symbol & $f_{s w, P S}$ & $L$ & $C$ & $T_{w}$ & \\
\hline Value & $20 \mathrm{kHz}$ & $100 \mu \mathrm{H}$ & $220 \mu \mathrm{F}$ & $1 \mathrm{~ms}$ & \\
\hline \hline
\end{tabular}

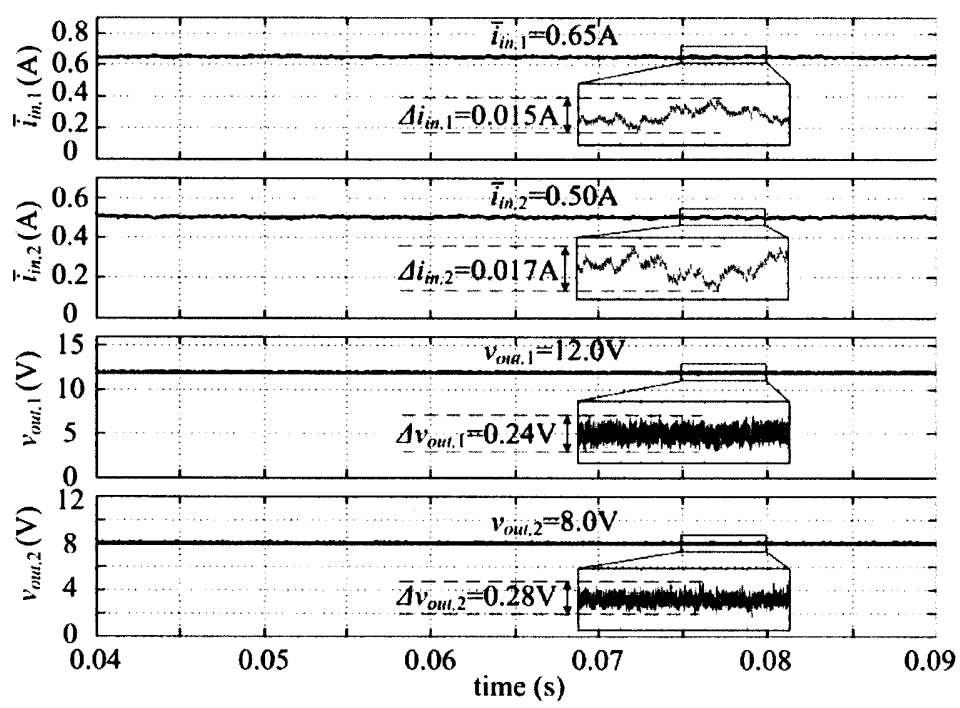

(a)

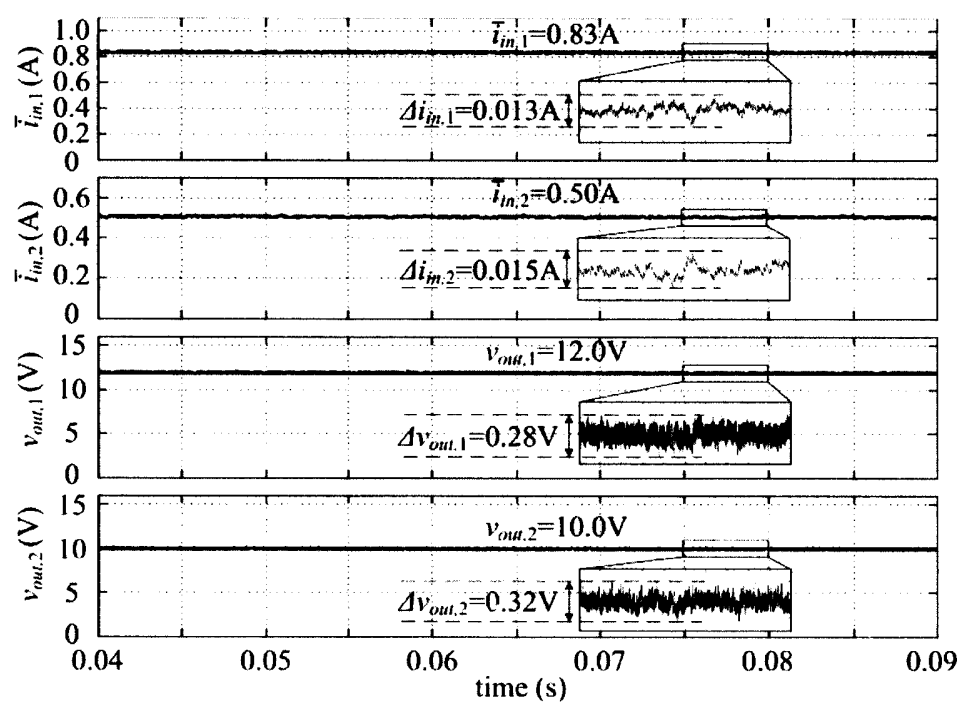

(b)

Figure 5-8: Simulation waveforms of $\bar{i}_{i n, 1}, \bar{i}_{i n, 2}, v_{o u t, 1}$ and $v_{o u t, 2}$ in steady-state operation of the proposed method in $v_{i n, 1}=24 \mathrm{~V}, v_{i n, 2}=20 \mathrm{~V}$ condition. (a) $v_{o u t, 1}=12.0 \mathrm{~V}$ and $v_{o u t, 2}=8.0 \mathrm{~V}$. (b) $v_{\text {out }, 1}=12.0 \mathrm{~V}$ and $v_{\text {out }, 2}=10.0 \mathrm{~V}$.

critical role in the values of $L$ and $C_{j}$. However, $f_{s w}$ of MPC method is varying as shown in Figure 5-6, which is different from the conventional methods. But an estimation of $f_{s w}$ is still possible. As the time step $t_{s}$ is set for discrete-time operation, the maximum value $f_{s w, \max }$ can be calculated as 


$$
f_{s w \text { max }}=\frac{1}{2 t_{s}}
$$

For robust consideration, the minimum value $f_{s w, \min }$ is defined and used in (5.19) and (5.20), which is set as $0.2 f_{s w, \max }$. Considering that $i_{L}$ is operated in a pseudo steady state, $\Delta i_{L}$ cannot be estimated by $0.3 i_{o u t, j}$ as estimated in the conventional methods in [134]. Since $i_{L}$ has the constraints in (5.11), $\Delta i_{L}$ is set as $(\beta-\alpha) I_{c r}$. Moreover, $\Delta v_{\text {out } j}$ is set as $5 \%$ $v_{o u t, j}$. Therefore, the values of inductor and capacitors can be determined with the information of $v_{i n, i}, v_{o u t, j}$ and $i_{o u t, j}$.

\subsection{Simulation ReSUlts}

In order to verify the performance of the proposed power sharing and cross regulation suppression method, simulation studies are conducted based on a SI-DIDO buck converter built in MATLAB/Simulink and the results are presented and discussed in this section.

The input voltage $v_{i n, i}$ has three values of $20,24,30 \mathrm{~V}$. The output voltage $v_{\text {out } j}$ also has three values of $8,10,12 \mathrm{~V}$, and the output current $i_{\text {out }, j}$ is $0.8 \sim 1.2 \mathrm{~A}$ considering load resistor $R_{j}$ is $8 \sim 10 \Omega$. Moreover, the time step $t_{s}$ is set as $5 \mu$ s so that the value of $f_{s w, \min }$ is $20 \mathrm{kHz}$ for robust consideration. By substituting these values and into (5.19) and assuming $\Delta i=4.5 \mathrm{~A}$, the maximum calculated value of inductor $L$ is $80 \mu \mathrm{H}$. In addition, considering the typical tolerance $(20 \%)$ of the inductance, the value of inductor is selected as $100 \mu \mathrm{H}$. Consequently, the maximum calculated value of the capacitor $C_{j}$ is $181 \mu \mathrm{F}$ based on (5.20). Similarly, considering the generally practical value and the typical tolerance $(20 \%)$ of the capacitance, the value of capacitor is selected as $220 \mu \mathrm{F}$. Furthermore, the switching frequency of $q_{P S, i}$ is defined as $f_{s w, P S}$ and set as $20 \mathrm{kHz}$. Simulation parameters of the SI-DIDO buck converter are listed in Table 5-1.

\subsubsection{Steady-state Operation}

The steady-state operation of the proposed method is studied in four conditions. Figure 5-8 shows the simulation results of steady-state operation of the proposed method when input voltages are $v_{i n, 1}=24 \mathrm{~V}, v_{i n, 2}=20 \mathrm{~V}$. In Figure 5-8(a), the output voltages are $v_{\text {out }, 1}=12.0 \mathrm{~V}$ and $v_{\text {out }, 2}=8.0 \mathrm{~V}$. It can be observed that the average values of input currents are $\bar{i}_{i n, 1}=0.65 \mathrm{~A}$ and $\bar{i}_{i n, 2}=0.50 \mathrm{~A}$. The voltage ripples are $\Delta v_{o u t, 1}=0.24 \mathrm{~V}$ and $\Delta v_{\text {out }, 2}=0.28 \mathrm{~V}$, i.e., $2 \%$ and $3.5 \%$ of the corresponding voltage references. The current ripples are $\Delta i_{i n, 1}=0.015 \mathrm{~A}$ and $\Delta i_{i n, 2}=0.017 \mathrm{~A}$, which is $3.4 \%$ of the current reference $\bar{i}_{\text {Ref: }}=0.50 \mathrm{~A}$. 
CHAPTER 5 POWER SHARGING AND CROSS REGULATION SUPPRESSION METHOD FOR SINGLE-INDUCTOR MULITPLE-INPUT MULTIPLE-OUTPUT DC-DC CONVERTER

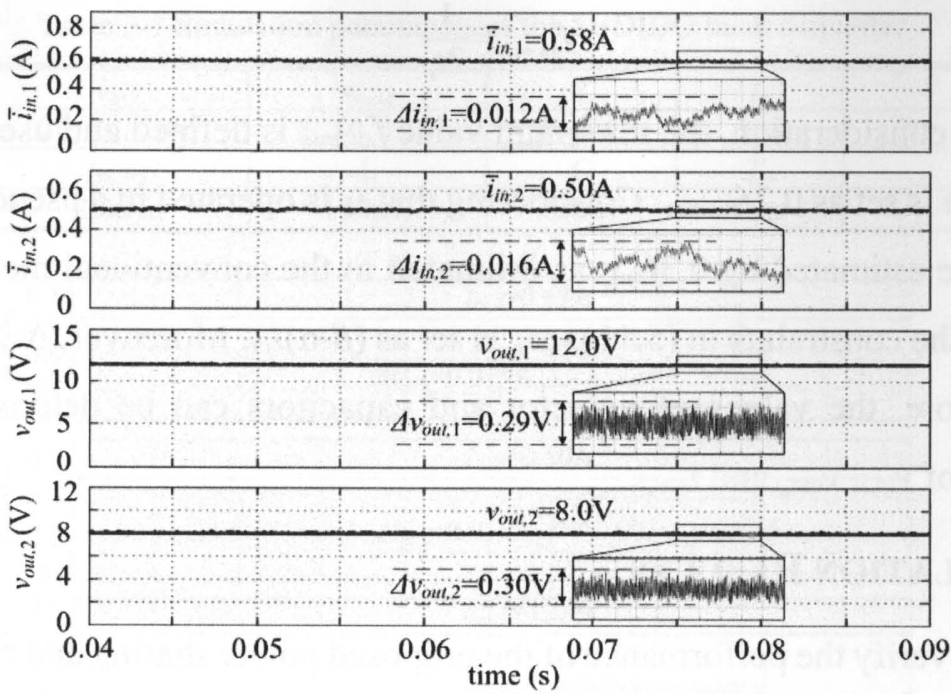

(a)
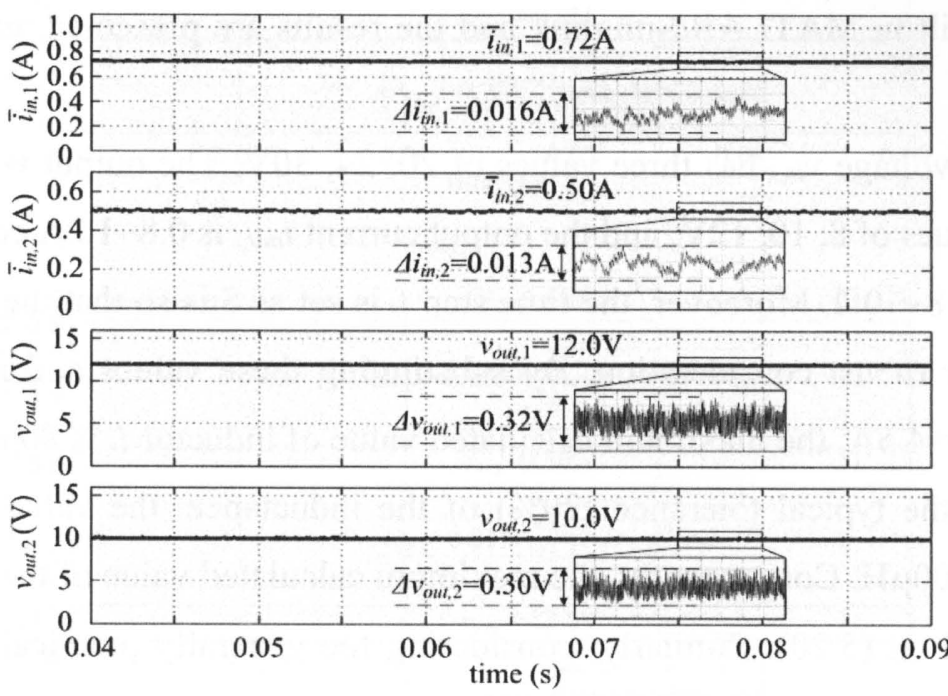

(b)

Figure 5-9: Simulation waveforms of $\bar{i}_{i n, 1}, \bar{i}_{i n, 2}, v_{\text {out }, 1}$ and $v_{\text {out }, 2}$ in steady-state operation of the proposed method under $v_{\text {in, }, 1}=30 \mathrm{~V}, v_{i n, 2}=20 \mathrm{~V}$ condition. (a) $v_{\text {out }, 1}=12.0 \mathrm{~V}$ and $v_{o u t, 2}=8.0 \mathrm{~V}$. (b) $v_{\text {out }, 1}=12.0 \mathrm{~V}$ and $v_{\text {out }, 2}=10.0 \mathrm{~V}$.

In Figure 5-8(b), the output voltages are $v_{\text {out }, 1}=12.0 \mathrm{~V}$ and $v_{\text {out }, 2}=10.0 \mathrm{~V}$. The average values of input currents are $\bar{i}_{i n, 1}=0.83 \mathrm{~A}$ and $\bar{i}_{i n .2}=0.50 \mathrm{~A}$. The voltage ripples are $\Delta v_{\text {out } .1}=0.28 \mathrm{~V}$ and $\Delta v_{\text {out }, 2}=0.32 \mathrm{~V}$, i.e., $2.3 \%$ and $3.2 \%$ of the corresponding voltage references. The current ripples are $\Delta i_{i n, 1}=0.013 \mathrm{~A}$ and $\Delta i_{i n, 2}=0.015 \mathrm{~A}$, which is $3 \%$ of the current reference $\bar{i}_{R_{R \in, 2}}=0.50 \mathrm{~A}$.

Similarly, the simulation results of steady-state operation in $v_{i n, 1}=30 \mathrm{~V}, v_{i n, 2}=20 \mathrm{~V}$ are shown in Figure 5-9. In Figure 5-9(a), the output voltages are $v_{o u t, 1}=12.0 \mathrm{~V}$ and $v_{\text {out }, 2}=8.0 \mathrm{~V}$. The average values of input currents are $\bar{i}_{i n, 1}=0.58 \mathrm{~A}$ and $\bar{i}_{i n, 2}=0.50 \mathrm{~A}$. The 


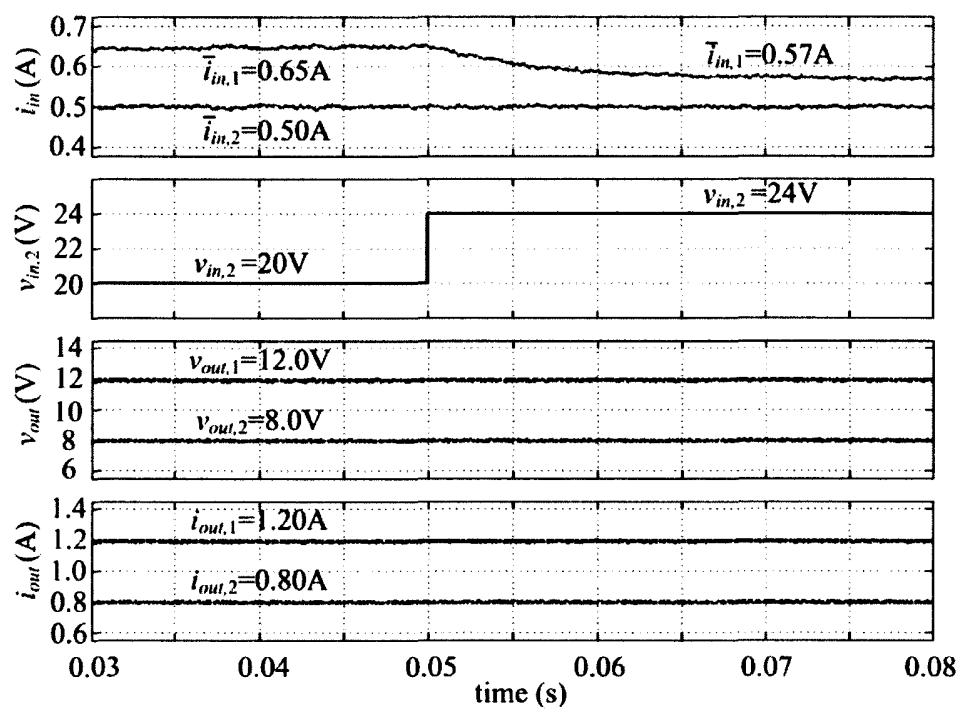

Figure 5-10: Line regulation simulation results of the proposed method.

voltage ripples are $\Delta v_{o u t, 1}=0.29 \mathrm{~V}$ and $\Delta v_{o u t, 2}=0.30 \mathrm{~V}$, i.e., $2.4 \%$ and $3.8 \%$ of the corresponding voltage references. The current ripples are $\Delta i_{i n, 1}=0.012 \mathrm{~A}$ and $\Delta i_{i n, 2}=0.016 \mathrm{~A}$, which is $3.2 \%$ of the current reference $\bar{i}_{i_{R e}, 2}=0.50 \mathrm{~A}$.

In Figure 5-9(b), the output voltages are $v_{\text {out }, 1}=12.0 \mathrm{~V}$ and $v_{\text {out }, 2}=10.0 \mathrm{~V}$, and the average values of input currents are $\bar{i}_{i n, 1}=0.72 \mathrm{~A}$ and $\bar{i}_{i n, 2}=0.50 \mathrm{~A}$. Moreover, the voltage ripples are $\Delta v_{o u t, 1}=0.32 \mathrm{~V}$ and $\Delta v_{o u t, 2}=0.30 \mathrm{~V}$, i.e.,, $2.7 \%$ and $3 \%$ of the corresponding voltage references. The current ripples are $\Delta i_{i n, 1}=0.016 \mathrm{~A}$ and $\Delta i_{i n, 2}=0.013 \mathrm{~A}$, which is $2.6 \%$ of the current reference $\bar{i}_{\text {Ref, } 2}=0.50 \mathrm{~A}$.

In summary, the proposed method is able to regulate the SI-DIDO buck converter following the input current reference and the output voltage references closely. Therefore, the power sharing and cross regulation suppression are both achieved. Comparing the results in Figure 5-8(a) and Figure 5-8(b) or Figure 5-9(a) and Figure 5-9(b), it can be concluded that less difference between $v_{o u t, 1}$ and $v_{o u t, 2}$ leads to better cross regulation suppression effect.

\subsubsection{Dynamic Performance}

The dynamic performance of the proposed method is studied including line regulation, load regulation, response to output voltage reference step change and response to input current reference step change. 

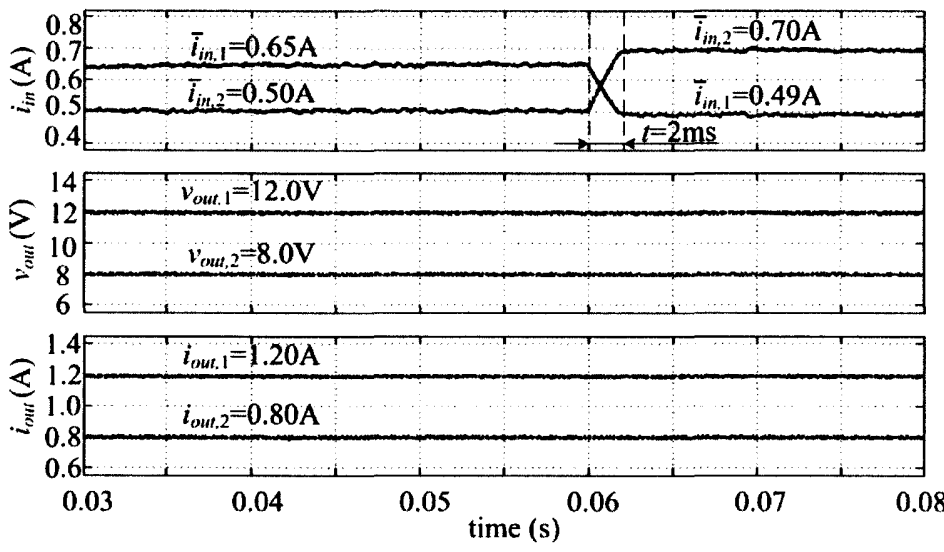

Figure 5-13: Simulation results of response to input current reference step change.

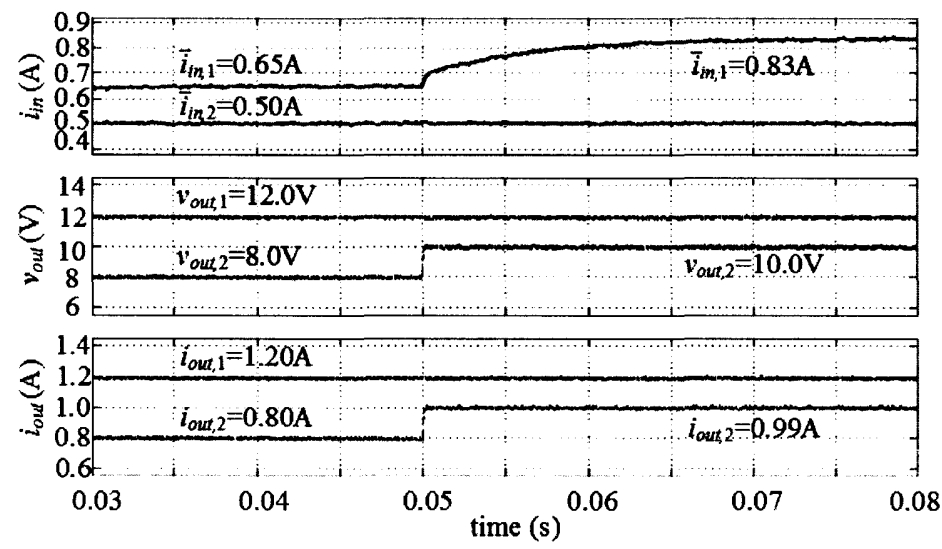

Figure 5-12: Simulation results of response to output voltage reference step change.

\subsubsection{Line Regulation}

The line regulation simulation results of the proposed method are shown in Figure 510. When $t=0.05 \mathrm{~s}$, the input voltage $v_{i n, 2}$ has a step change from $20 \mathrm{~V}$ to $24 \mathrm{~V}$. The average current $\bar{i}_{i, 2}$ is regulated to follow the preset reference $0.5 \mathrm{~A}$ closely by the proposed method with control signals $\left(q_{P S, 1}, q_{P S, 2}\right)$.

Consequently, $\bar{i}_{i n 1}$ is decreased from $0.65 \mathrm{~A}$ to $0.57 \mathrm{~A}$ as the input power from second input source is increased. Because the MAF is applied to calculate the average values of input currents, the waveforms of $\bar{i}_{i, 1}$ and $\bar{i}_{m, 2}$ are smooth and vary slowly, and the transient response (overshoot or undershoot) is eliminated. Moreover, the output voltages $v_{o u t, 1}$ and $v_{\text {out }, 2}$ are $12.0 \mathrm{~V}$ and $8.0 \mathrm{~V}$ respectively before and after the input voltage $v_{i n, 2}$ step change controlled by the dynamic signals $\left(q_{1}, q_{2}\right)$ and $\left(q_{1}^{\prime}, q_{2}{ }^{\prime}\right)$. Hence, 
$\bar{i}_{i, 2}, v_{o u t, 1}$ and $v_{o u t, 2}$ in simulation are merely influenced by the step change in input voltage so that the influence is barely observed from the waveforms.

\subsubsection{Load Regulation}

The load regulation simulation results of the proposed method are shown in Figure 5-11. The load $R_{2}$ is changed from $10 \Omega$ to $8.24 \Omega$ when $t=0.05 \mathrm{~s}$, and $i_{\text {out }, 2}$ is increased from $0.8 \mathrm{~A}$ to $0.96 \mathrm{~A}$ accordingly, i.e., a $20 \%$ load increment. Moreover, the control signals $\left(q_{P S, 1}, q_{P S, 2}\right)$ are regulated to increase $\bar{i}_{i, 1}$ from $0.65 \mathrm{~A}$ to $0.73 \mathrm{~A}$ to satisfy the load increment, while maintain $\bar{i}_{i, 2}$ to follow the current reference 0.5A. Similarly, the transient responses of $\bar{i}_{i, 1}$ and $\bar{i}_{i, 2}$ are eliminated by the MAF. The output voltages $v_{o u t, 1}$ and $v_{o u t, 2}$ are $12.0 \mathrm{~V}$ and $8.0 \mathrm{~V}$ respectively before and after the load step change, which means that the proposed method significantly reduce the influence of load step change on the output voltages.

\subsubsection{Response to Output Voltage Reference Step Change}

Figure 5-12 shows the simulation results of response to output voltage reference step change. It can be seen that the output voltage $v_{\text {out }, 2}$ has a step change from $8.0 \mathrm{~V}$ to $10.0 \mathrm{~V}$ when $t=0.05 \mathrm{~s}$, and the output current $i_{\text {out }, 2}$ is increased from $0.8 \mathrm{~A}$ to $0.99 \mathrm{~A}$. Since the input current $\bar{i}_{i, 2}$ is fixed at $0.50 \mathrm{~A}$ by the proposed method, $\bar{i}_{i n, 1}$ is increased from 0.65 to $0.83 \mathrm{~A}$ to meet the increased power demand. For the output voltage $v_{o u t, 1}$, it is regulated as $12.0 \mathrm{~V}$ and barely influenced by the step change of $v_{\text {out }, 2}$ in simulation. 


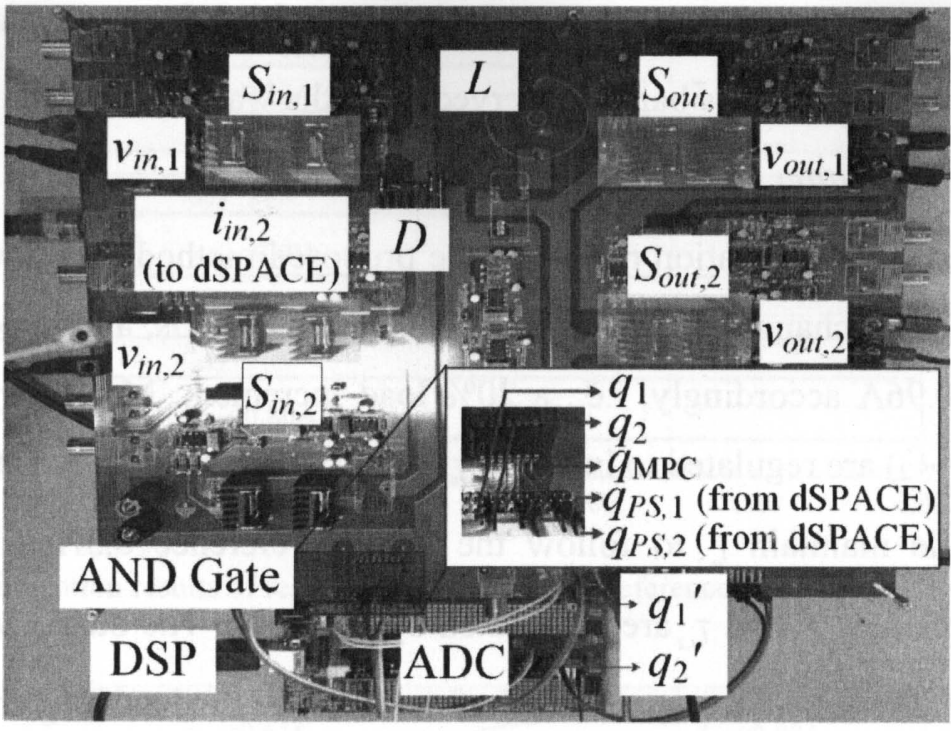

Figure 5-14: Hardware platform for verification of the proposed method.

Table 5-2 Main components adopted in hardware platform

\begin{tabular}{l|l|l}
\hline \hline Component & Device & Performance parameters \\
\hline MOSFET & IRF540 & Rise time $44 \mathrm{~ns}$, fall time $43 \mathrm{~ns}$ \\
\hline MOSFET driver & HCPL-J314 & Propagation delay $0.7 \mu \mathrm{s}$, rise time $50 \mathrm{ns,} \mathrm{fall} \mathrm{time} 50 \mathrm{~ns}$ \\
\hline AND gates & HD74HC08 & Propagation delay $23 \mathrm{~ns}$, rise time $19 \mathrm{~ns}$, fall time $19 \mathrm{~ns}$ \\
\hline Diode & MBRB60H100CT & Forward voltage $0.72 \mathrm{~V}$ \\
\hline Inductor & $1140-101 \mathrm{~K}-\mathrm{RC}$ & Current rating $I_{c r}=10.4 \mathrm{~A}$ \\
\hline Current transducer & LAH 25-NP & Response time $0.5 \mathrm{us}$ \\
\hline Voltage transducer & LV 25-P & Response time $40 \mu \mathrm{s}$ \\
\hline DSP controller & TI TMS320F28335 & Operation frequency $150 \mathrm{MHz}$ \\
\hline dSPACE & DS 1104 Board & Operation frequency $250 \mathrm{MHz}$ \\
\hline \hline
\end{tabular}

\subsubsection{Response to Input Current Reference Step Change}

The simulation results of response to input current reference step change are shown in Figure 5-13. A change is set for the input current reference $\bar{i}_{\text {Ref }}$ from $0.5 \mathrm{~A}$ to $0.7 \mathrm{~A}$ when $t=0.06 \mathrm{~s}$, and it can be seen that it takes about $2 \mathrm{~ms}$ for the proposed method to regulate $\bar{i}_{i, 2}$ from $0.50 \mathrm{~A}$ to $0.70 \mathrm{~A}$. Moreover, $\bar{i}_{i, 1}$ is decreased from $0.65 \mathrm{~A}$ to $0.49 \mathrm{~A}$ to maintain the output powers from the sources. It can be seen that $v_{o u t, 1}$ and $v_{o u t, 2}$ are regulated to follow the preset references closely even the two average currents from two input sources have changed. 
CHAPTER 5 POWER SHARGING AND CROSS REGULATION SUPPRESSION METHOD FOR SINGLE-INDUCTOR MULITPLE-INPUT MULTIPLE-OUTPUT DC-DC CONVERTER

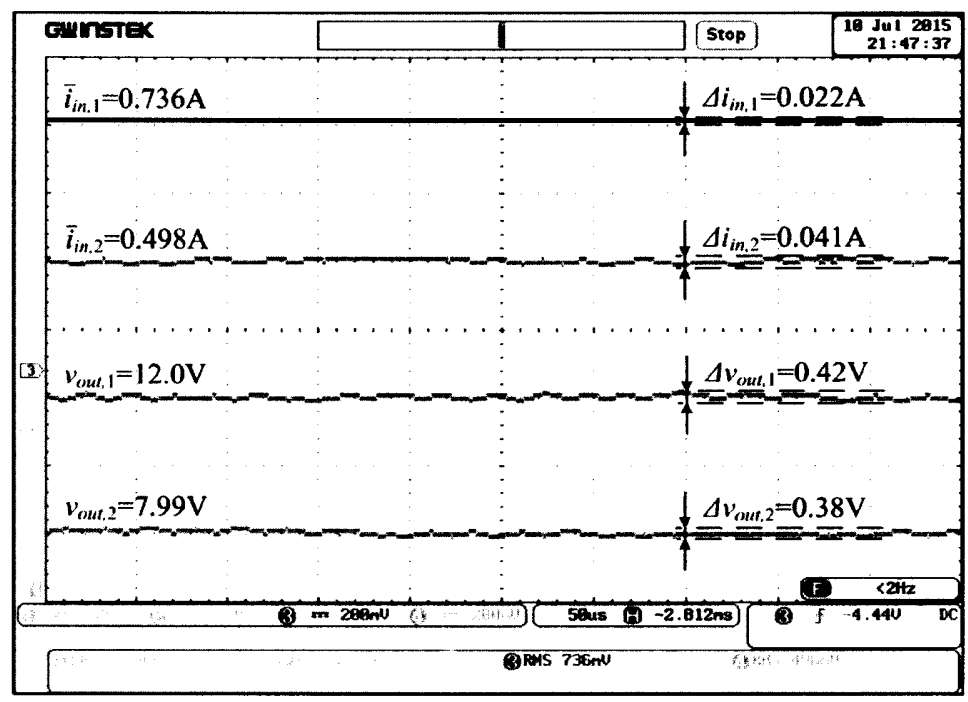

(a)

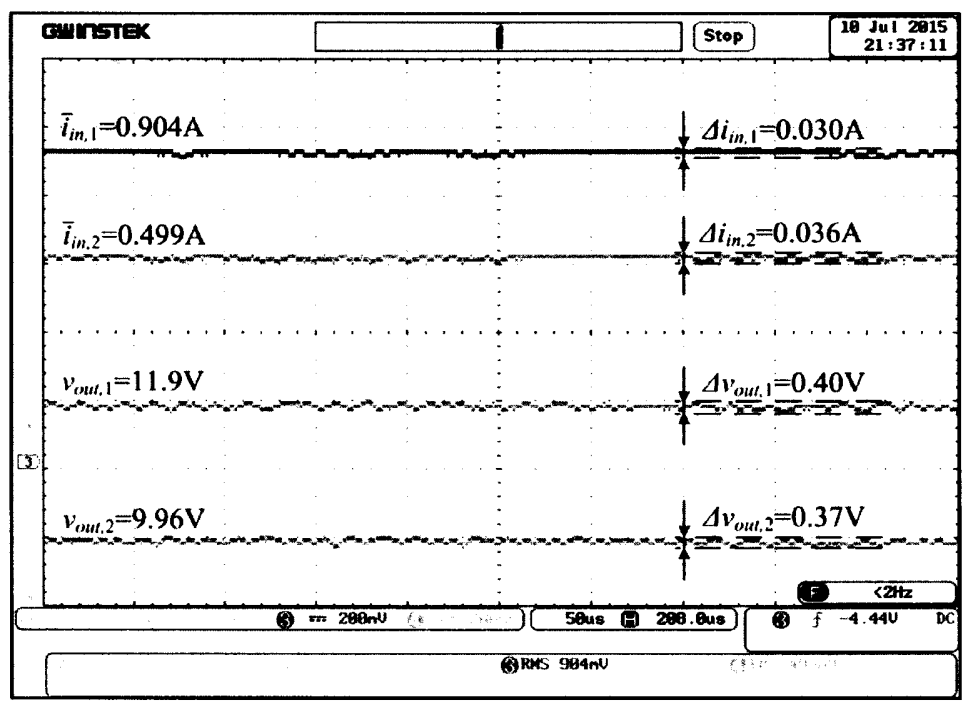

(b)

Figure 5-15: Experimental waveforms of $\bar{i}_{i n, 1}, \bar{i}_{i n, 2}, v_{o u t, 1}$ and $v_{o u t, 2}$ in steady-state operation of the proposed method under $v_{i n, 1}=24 \mathrm{~V}, v_{i n, 2}=20 \mathrm{~V}$ conditions based on the SI-DIDO buck converter. (a) $v_{\text {out }, 1}=12.0 \mathrm{~V}$ and $v_{\text {out }, 2}=7.99 \mathrm{~V}$. (b) $v_{\text {out }, 1}=11.9 \mathrm{~V}$ and $v_{\text {out }, 2}=9.96 \mathrm{~V}$.

\subsection{EXPERIMENTAL Results}

In this section, an experimental prototype of SI-DIDO buck converter, as shown in Figure $5-14$ by setting $m=n=2$, is built to verify the proposed power sharing and cross regulation suppression method including steady-state operation and dynamic performance. The hardware platform consists of the SI-DIDO buck converter, a dSPACE controller board as the power sharing controller and a DSP controller as the cross regulation suppression controller. The components adopted in the hardware 
CHAPTER 5 POWER SHARGING AND CROSS REGULATION SUPPRESSION METHOD FOR SINGLE-INDUCTOR MULITPLE-INPUT MULTIPLE-OUTPUT DC-DC CONVERTER

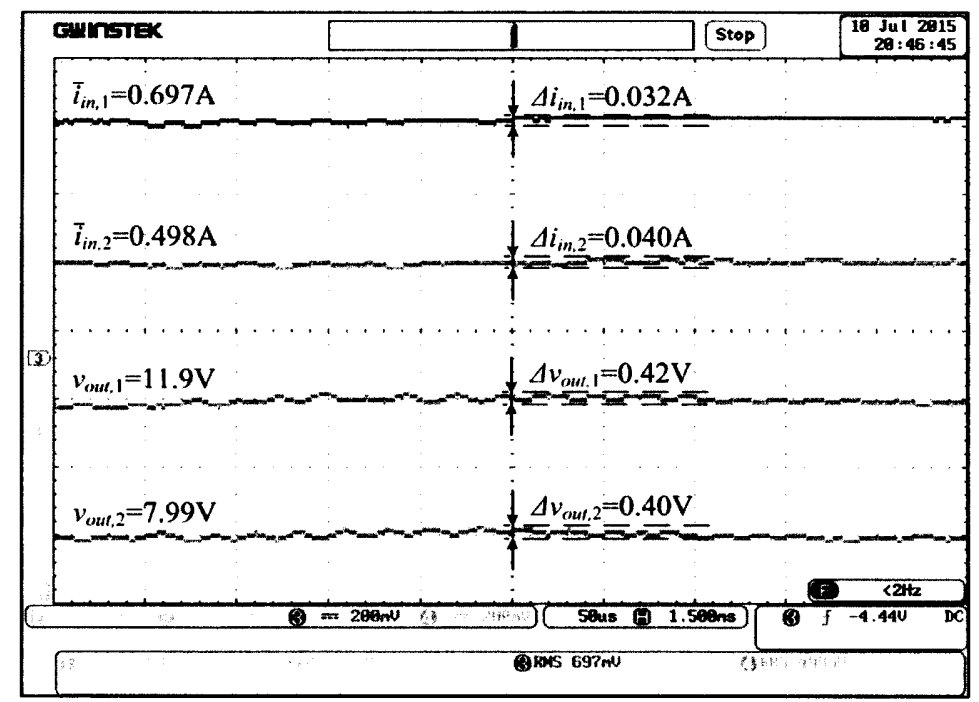

(a)

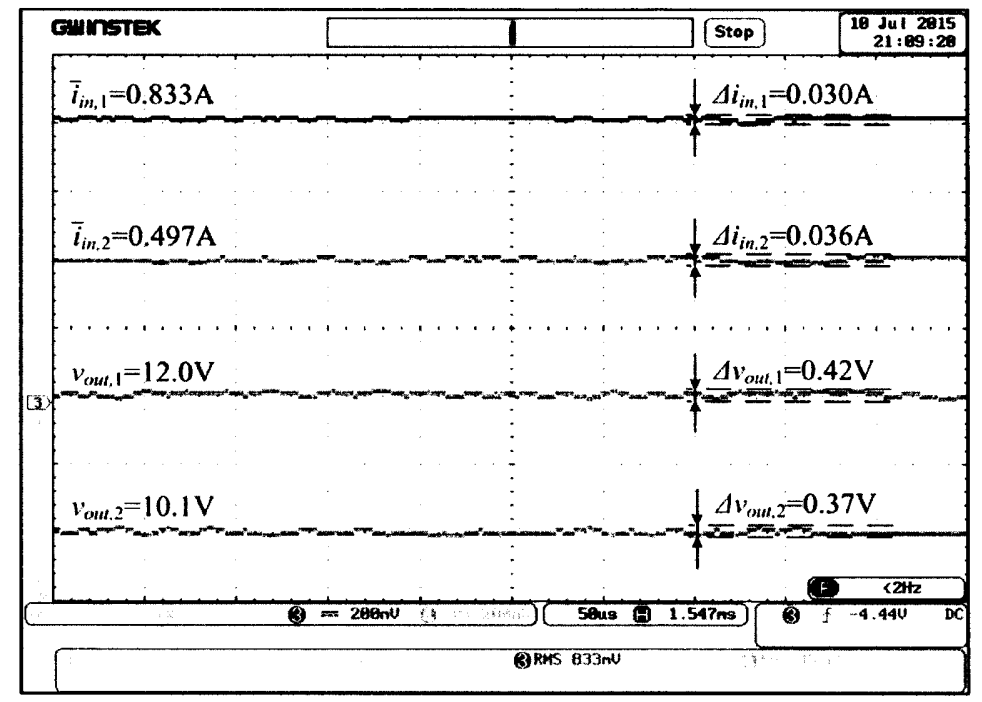

(b)

Figure 5-16: Experimental waveforms of $\bar{i}_{i, 1}, \bar{i}_{i n, 2}, v_{o u t, 1}$ and $v_{o u t, 2}$ in steady-state operation of the proposed method under $v_{i n, 1}=30 \mathrm{~V}, v_{i n, 2}=20 \mathrm{~V}$ ccndition. (a) $v_{o u t, 1}=11.9 \mathrm{~V}$ and $v_{o u t, 2}=7.99 \mathrm{~V}$. (b) $v_{\text {out }, 1}=12.0 \mathrm{Vand} v_{\text {out }, 2}=10.1 \mathrm{~V}$.

platform and the corresponding performance parameters are shown in Table 5-2. The other parameters are shown in the Table 5-1. Moreover, the constraint coefficients of $i_{L}$ are set as $\alpha=1 / 25$ and $\beta=1 / 2$ in the experiment. Therefore, the tolerable minimum and maximum values of the inductor current $i_{L}$ are $i_{L, \min }=0.416 \mathrm{~A}$ and $i_{L, \max }=5.2 \mathrm{~A}$.

\subsubsection{Steady-state Operation}

The experimental results of steady-state operation based on the SI-DIDO buck converter hardware prototype are presented and discussed in this subsection. 


\subsubsection{Steady-state Operation Waveforms}

Figure 5-15 shows experimental waveforms with input voltages $v_{i n, 1}=24 \mathrm{~V}$ and $v_{i n, 2}=20 \mathrm{~V}$. In Figure 5-15(a), the output voltages are $v_{o u t, 1}=12.0 \mathrm{~V}$ and $v_{o u t, 2}=7.99 \mathrm{~V}$, and the average values of input currents are $\bar{i}_{i n .1}=0.736 \mathrm{~A}$ and $\bar{i}_{i, 2}=0.498 \mathrm{~A}$. The voltage ripples are $\Delta v_{\text {out }, 1}=0.42 \mathrm{~V}$ and $\Delta v_{\text {out }, 2}=0.38 \mathrm{~V}$, i.e., $3.5 \%$ and $4.75 \%$ of the corresponding voltage references. The current ripples are $\Delta i_{i n, 1}=0.022 \mathrm{~A}$ and $\Delta i_{i n, 2}=0.041 \mathrm{~A}$, which is $8.2 \%$ of the current reference $\bar{i}_{\text {Ref }, 2}=0.50 \mathrm{~A}$. In Figure 5-15(b), the output voltages are $v_{\text {out }, 1}=11.9 \mathrm{~V}$ and $v_{\text {out }, 2}=9.96 \mathrm{~V}$. The average values of input currents are $\bar{i}_{i n, 1}=0.904 \mathrm{~A}$ and $\bar{i}_{i n, 2}=0.499 \mathrm{~A}$. The voltage ripples are $\Delta v_{\text {out }, 1}=0.40 \mathrm{~V}$ and $\Delta v_{\text {out }, 2}=0.37 \mathrm{~V}$, i.e., $3.4 \%$ and $3.7 \%$ of the corresponding voltage references. The current ripples are $\Delta i_{i n, 1}=0.03 \mathrm{~A}$ and $\Delta i_{i n, 2}=0.036 \mathrm{~A}$, which is $7.2 \%$ of the current reference $\bar{i}_{\text {Ret }, 2}=0.50 \mathrm{~A}$.

In Figure 5-16, the input voltages are $v_{i n, 1}=30 \mathrm{~V}$ and $v_{i n, 2}=20 \mathrm{~V}$. In Figure 5-16(a), the output voltages are $v_{\text {out }, 1}=11.9 \mathrm{~V}$ and $v_{\text {out }, 2}=7.99 \mathrm{~V}$. The average values of input currents are $\bar{i}_{i n, 1}=0.697 \mathrm{~A}$ and $\bar{i}_{i, 2}=0.498 \mathrm{~A}$. Moreover, the voltage ripples are $\Delta v_{o u t, 1}=0.42 \mathrm{~V}$ and $\Delta v_{o u t, 2}=0.40 \mathrm{~V}$, i.e., $3.5 \%$ and $5 \%$ of the corresponding voltage references. The current ripples are $\Delta i_{i n, 1}=0.032 \mathrm{~A}$ and $\Delta i_{i n, 2}=0.04 \mathrm{~A}$, which is $8 \%$ of the current reference $\bar{i}_{\text {Ret }, 2}=0.50 \mathrm{~A}$. In Figure 5-16(b), the output voltages are $v_{o u t, 1}=12.0 \mathrm{~V}$ and $v_{o u t, 2}=10.1 \mathrm{~V}$. The average input currents are $\bar{i}_{i n, 1}=0.833 \mathrm{~A}$ and $\bar{i}_{i n, 2}=0.497 \mathrm{~A}$. Furthermore, the voltage ripples are $\Delta v_{\text {out }, 1}=0.42 \mathrm{~V}$ and $\Delta v_{o u t, 2}=0.37 \mathrm{~V}$, i.e., $3.5 \%$ and $3.7 \%$ of the corresponding voltage references. The current ripples are $\Delta i_{i n, 1}=0.030 \mathrm{~A}$ and $\Delta i_{i n, 2}=0.036 \mathrm{~A}$, which is $7.2 \%$ of the current reference $\bar{i}_{\text {Re, }, 2}=0.50 \mathrm{~A}$.

The experimental results demonstrate that the proposed method is able to achieve the power sharing and cross regulation suppression for the SI-DIDO buck converter. Moreover, it can be concluded that less difference between $v_{o u t, 1}$ and $v_{o u t, 2}$ results in better cross regulation suppression effect by comparing the results in Figure 5-15(a) and Figure 5-15(b) or Figure 5-16(a) and Figure 5-16(b).

\subsubsection{Control Signal Waveforms at Steady-state Operation}

In Figure 5-17, the experimental waveforms of the control signals $q_{P S, 1}, q_{P S, 2}, q_{1}{ }^{\prime}, q_{1}$ and $q_{2}$, and inductor current $i_{L}$ in steady-state operation under $v_{i n, 1}=24 \mathrm{~V}, v_{i n, 2}=20 \mathrm{~V}$, $v_{\text {out }, 1}=12.0 \mathrm{~V}, v_{\text {out }, 2}=8.0 \mathrm{~V}$ condition are presented to explain the operation of the proposed method. 


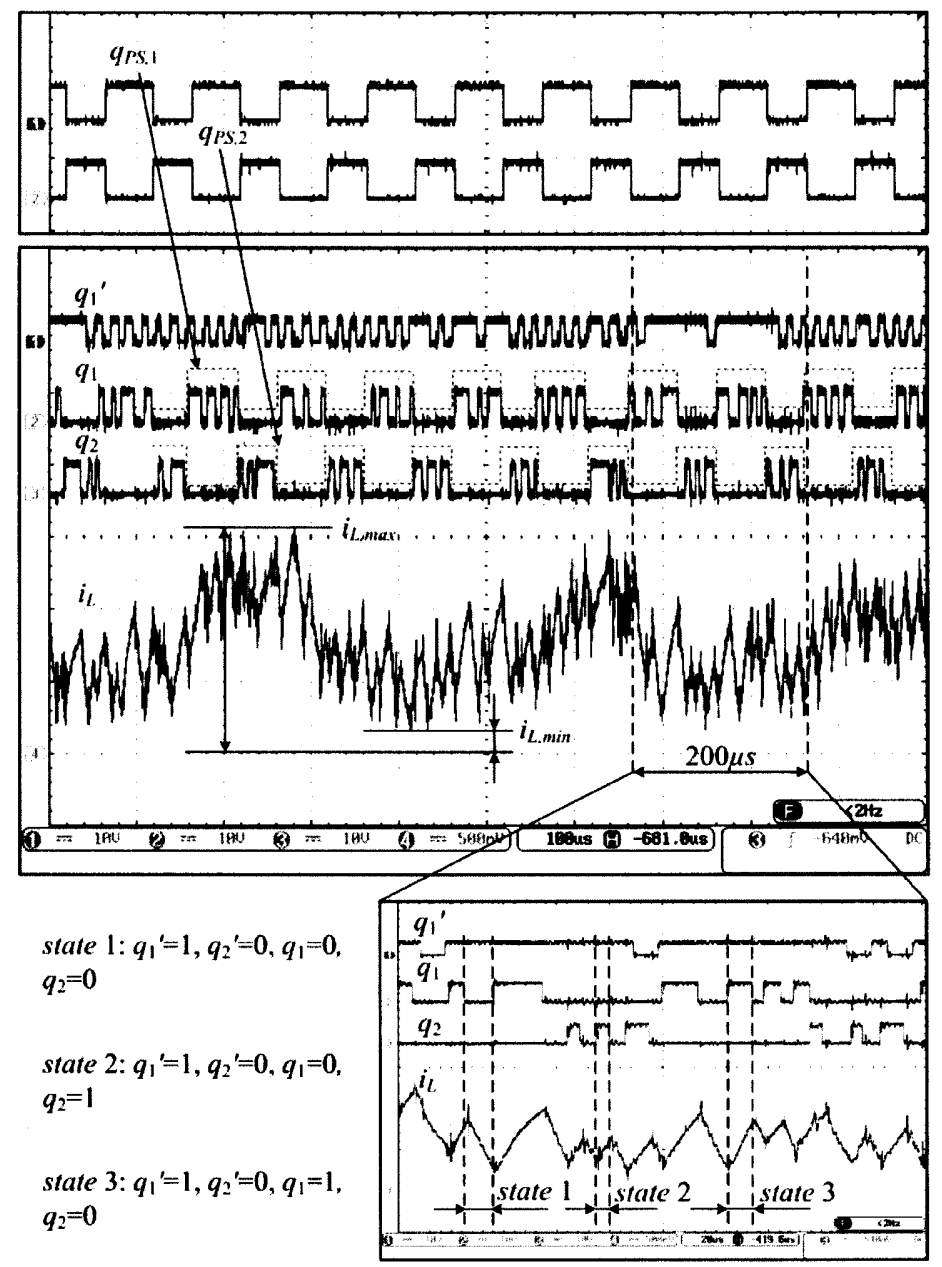

Figure 5-17: Waveforms of control signals $q_{P S, 1}, q_{P S, 2}, q_{1}$ ', $q_{1}$ and $q_{2}$ and inductor current $i_{l}$ under $v_{\text {in }, 1}=24 \mathrm{~V}, v_{\text {in, }, 2}=20 \mathrm{~V}, v_{\text {out }, 1}=12.0 \mathrm{~V}$ and $v_{\text {out }, 2}=8.0 \mathrm{~V}$ condition.

In the top figure of Figure 5-17, it can be seen that $q_{P S, 1}$ is the envelope curves of the control signals $q_{1}$, which means that $q_{1}$ is the logical conjunction of $q_{P S, 1}$ generated by the power sharing method and $q_{M P C}$ generated by the MPC method. Similarly, $q_{2}$ is the result of logical conjunction of $q_{P S, 2}$ and $q_{M P C} q_{1}$ ' is the selection signal and generated by the time-multiplexing method, $q_{2}$ ' is not shown because it is the complementation of $q_{1}$ ' and the oscilloscope only has four channels. These signals are dynamically controlled by the proposed method based on the real-time data and preset references so that the power sharing and cross regulation suppression are both realized. Moreover, it can be seen that the inductor current $i_{L}$ has a maximum value $i_{L, m a x}$ and a minimum value $i_{L, \min }$ which follow the preset constraints. Therefore, it guarantees the CCM operation of the SI-DIDO buck converter and the effectiveness of the inductor. Actually, the inductor current $i_{L}$ waveform is the voltage signal $V_{I L}$ from the current transducer LAH 
25-NP, and the relationship between $i_{L}$ and $V_{I L}$ is described by a linear equation as follows:

$$
i_{L}=2.513 \times V_{L L}-0.0357
$$

Therefore, the maximum value $i_{L, \max }$ and the minimum value $i_{L, \min }$ are $3.86 \mathrm{~A}$ and $0.42 \mathrm{~A}$ respectively, based on the maximum value of $1.55 \mathrm{~V}$ and minimum value of $180 \mathrm{mV}$ of $V_{I L}$. Hence, the inductor current $i_{L}$ follows the preset constraints well.

A zoom-in view of the control signals $q_{1}{ }^{\prime}, q_{1}$ and $q_{2}$, and inductor current $i_{L}$ are shown in the bottom of the Figure 5-17. Three states are selected for further explanation of the steady-state operation. In these three states, $q_{1}^{\prime}=1$ and $q_{2}^{\prime}=0$ means that the first branch is selected for supply. In state $1, q_{1}=0$ and $q_{2}=0$, the inductor is discharged so that the waveform of $i_{L}$ is decreasing.

In state $2, q_{1}=0$ and $q_{2}=1$, the inductor is charged by the second input source so that the waveform of $i_{L}$ is rising. Similarly, the inductor is charged by the first input source in state 3 . The different slopes of $i_{L}$ are caused by the different input voltages and output voltages. Therefore, the dynamic control signals generated by the proposed method contribute the capability of power sharing and cross regulation suppression.

\subsubsection{Dynamic Performance}

The experimental results of the dynamic performance based on the SI-DIDO buck converter hardware prototype are presented and discussed in this subsection.

\subsubsection{Line Regulation}

The line regulation experimental results of the proposed method are shown in Figure $5-18$. It can be seen that the input voltage $v_{i n, 2}$ has a step change from $20 \mathrm{~V}$ to $24 \mathrm{~V}$. The proposed method will dynamically regulate the control signals $\left(q_{P S, 1}, q_{P S, 2}\right)$ to keep $\bar{i}_{m, 2}$ as $0.501 \mathrm{~A}$, which follows the preset reference $0.5 \mathrm{~A}$ well. Consequently, $\bar{i}_{i, 1}$ is finally decreased from $0.740 \mathrm{~A}$ to $0.642 \mathrm{~A}$ because the input power from first input source is increased. As the MAF is applied to calculate $\bar{i}_{i, 1}$ and $\bar{i}_{i_{n, 2}}$, the corresponding waveforms of $\bar{i}_{i, 1}$ and $\bar{i}_{i, 2}$ are smooth, and the transient response is eliminated.

Moreover, the $\left(q_{1}, q_{2}\right)$ and $\left(q_{1}^{\prime}, q_{2}^{\prime}\right)$ are dynamically controlled so that the output voltages $v_{o u t, 1}$ and $v_{o u t, 2}$ are equal to $12.0 \mathrm{~V}$ and $7.98 \mathrm{~V}$ respectively before the input voltage $v_{i n, 2}$ step change, which track the voltage references $12 \mathrm{~V}$ and $8 \mathrm{~V}$ closely. The zoom-in views of $i_{\text {out }, 1}, i_{\text {out }, 2}, v_{\text {out }, 1}$ and $v_{\text {out }, 2}$ are also shown in Figure 5-18. It can be seen 


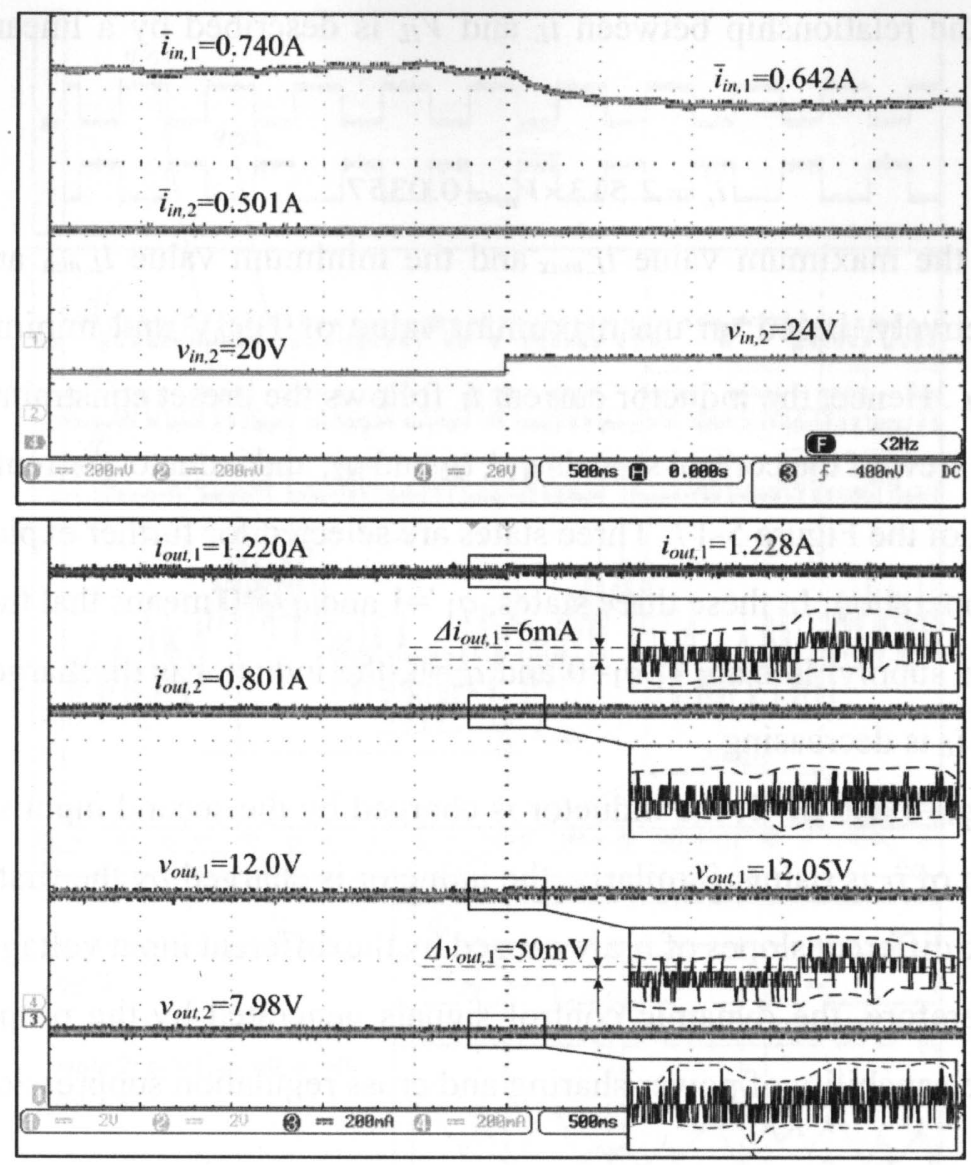

Figure 5-18: Experimental results of line regulation of the proposed method based on SI-DIDO buck converter.

that there is a small increment of $50 \mathrm{mV}$ in $v_{o u t, 1}$, and an increment of $8 \mathrm{~mA}$ in $i_{\text {out }, 1}$ correspondingly. Therefore, the line regulation is well performed.

\subsubsection{Load Regulation}

The load regulation experimental results of the proposed method are shown in Figure 5-19. The load $R_{2}$ has a step change from $10 \Omega$ to $8.24 \Omega$, and the $i_{o u t, 2}$ is increased from $0.801 \mathrm{~A}$ to $0.981 \mathrm{~A}$ correspondingly, i.e., a $22 \%$ load increment. In order to meet this load step change, the control signals $\left(q_{P S, 1}, q_{P S, 2}\right)$ are regulated to increase $\bar{i}_{i, 1}$ from $0.725 \mathrm{~A}$ to $0.822 \mathrm{~A}$ for higher load demand, while maintain $\bar{i}_{i_{1,2}}$ around the reference $0.5 \mathrm{~A}$. Similarly, the transient response of $\bar{i}_{i, 1}$ and $\bar{i}_{m, 2}$ are filtered by the MAF.

The output voltage $v_{\text {out }, 1}$ and $v_{\text {out }, 2}$ are regulated to $12.0 \mathrm{~V}$ and $8.0 \mathrm{~V}$ respectively before the load step change, which means $v_{o u t, 1}$ and $v_{\text {out }, 2}$ track the voltage references successfully. The zoom-in views of $i_{\text {out }, 1}, i_{\text {out }, 2}, v_{\text {out }, 1}$ and $v_{\text {out }, 2}$ are also shown in the Figure 5-18. It can be observed that there are small decrements of $45 \mathrm{mV}$ in $v_{\text {out }, 1}$ and 

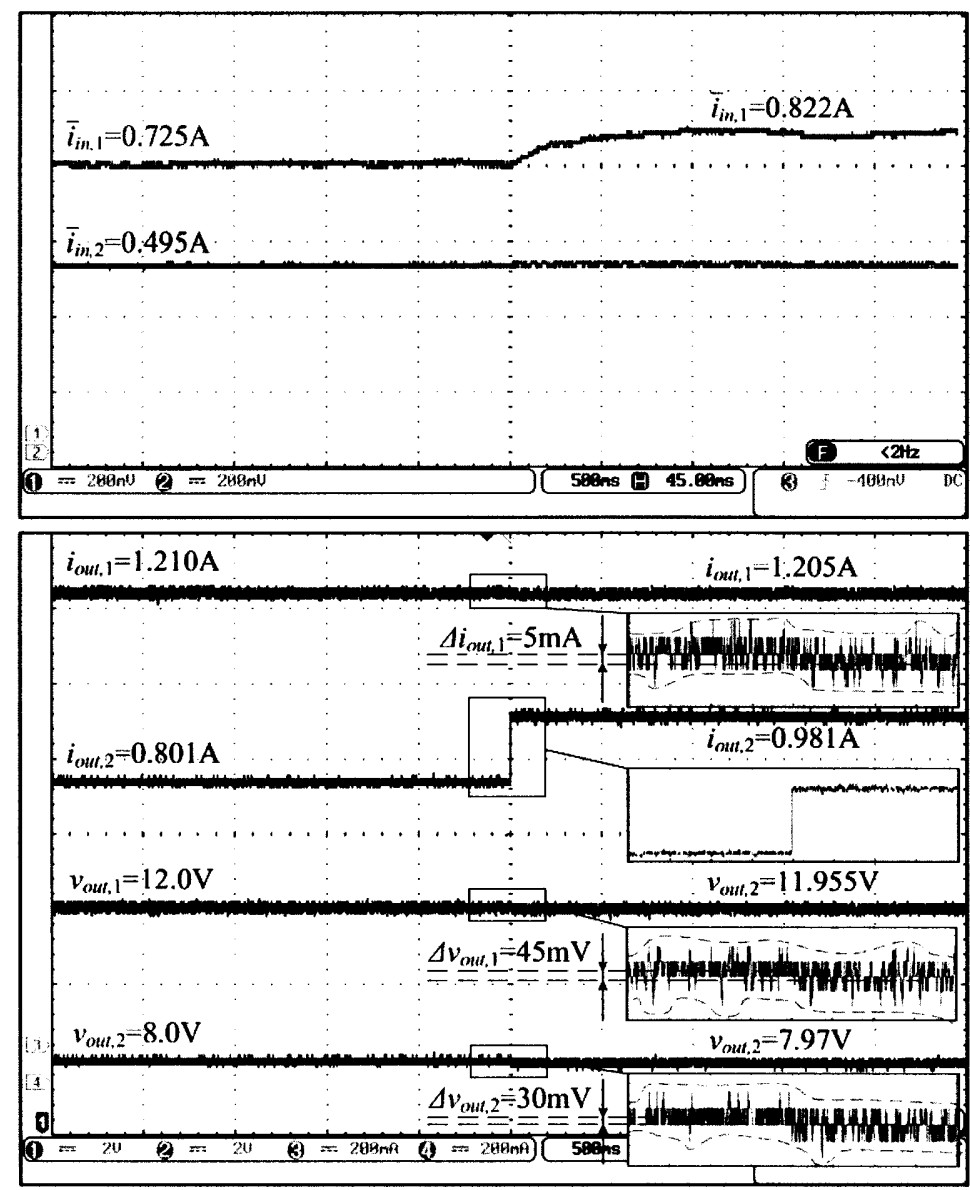

Figure 5-19: Experimental results of load regulation of the proposed method based on the SI-DIDO buck converter.

$30 \mathrm{mV}$ in $v_{\text {out }, 2}$ respectively, and a decrement of $5 \mathrm{~mA}$ in $i_{\text {out }, 1}$, which demonstrate that the cross regulation is suppressed.

\subsubsection{Response to Output Voltage Reference Step Change}

The experimental results of response to output voltage reference step change are shown in Figure 5-20. It can be seen that the output voltage $v_{\text {out }, 2}$ has a step change from $8.0 \mathrm{~V}$ to $10.1 \mathrm{~V}$. Since the input current $\bar{i}_{i, 2}$ is regulated to $0.494 \mathrm{~A}$ following the preset current reference $0.5 \mathrm{~A}, \bar{i}_{i, 1}$ is increased from $0.729 \mathrm{~A}$ to $0.910 \mathrm{~A}$ to meet the increased power demand. The transient responses of $\bar{i}_{i, 1}$ and $\bar{i}_{i, 2}$ are eliminated by the MAF. The power sharing is completed by the dynamic adjustment of the control signals ( $q_{P S, 1}$, $q_{P S, 2) \text {. }}$

The zoom-in views of $i_{\text {out }, 1}, i_{\text {out }, 2}, v_{\text {out }, 1}$ and $v_{\text {out }, 2}$ are also shown in the Figure 5-20. It can be observed that there is a small decrement of $50 \mathrm{mV}$ in $v_{\text {out }, 1}$, and a small increment of $4 \mathrm{~mA}$ in $i_{\text {out }, 1}$ correspondingly, which means that the cross regulation is significantly 
CHAPTER 5 POWER SHARGING AND CROSS REGULATION SUPPRESSION METHOD FOR SINGLE-INDUCTOR MULITPLE-INPUT MULTIPLE-OUTPUT DC-DC CONVERTER

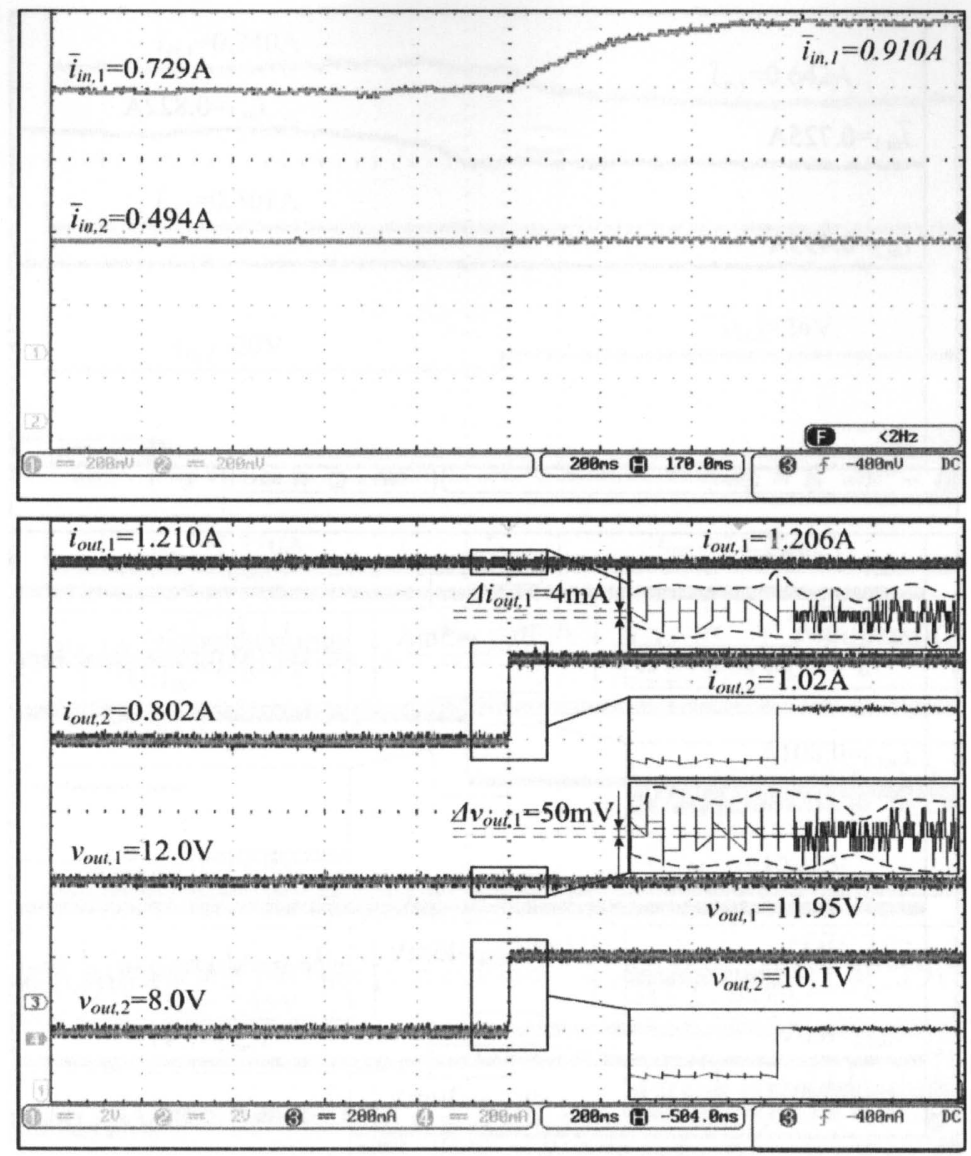

Figure 5-20: Experimental results of response to output voltage reference step change.

suppressed. The step change in the output voltage reference $v_{R e f, 2}$ leads to the second load $R_{2}$ has a larger priority for a certain time slot so that $v_{o u t, 1}$ will be allowed to freely decrease during this time slot until it is back to higher priority. The envelope of $v_{\text {out }, 1}$ shows that it recovers back to $11.95 \mathrm{~V}$ after $v_{o u t, 2}$ completing the change from $8.0 \mathrm{~V}$ to $10.1 \mathrm{~V}$.

\subsubsection{Response to Input Current Reference Step Change}

The experimental results of response to input current reference step change are shown in Figure 5-21. A change is set for the input current $\bar{i}_{i, 2}$ from $0.5 \mathrm{~A}$ to $0.7 \mathrm{~A}$, and it can be seen that it takes about $85 \mathrm{~ms}$ for the proposed method to regulate $\bar{i}_{i, 2}$ from $0.495 \mathrm{~A}$ to $0.691 \mathrm{~A}$. Accordingly, $\bar{i}_{i, 1}$ is decreased from $0.730 \mathrm{~A}$ to $0.572 \mathrm{~A}$ to maintain the output power from the sources. These smooth waveforms of $\bar{i}_{i, 1,1}$ and $\bar{i}_{i, 2}$ result from the usage of the MAF. 


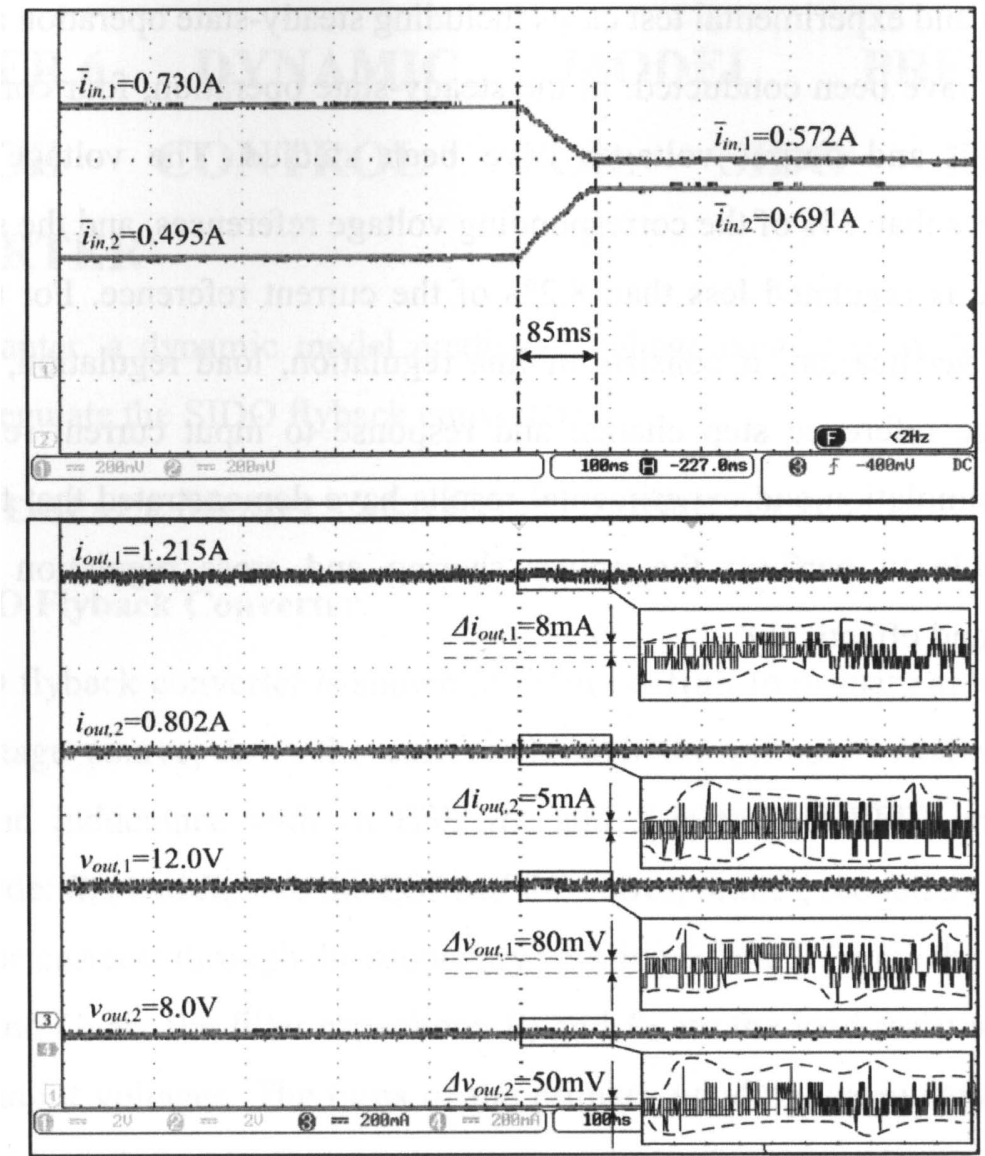

Figure 5-21: Experimental results of response to input current reference step change.

The zoom-in views of $i_{\text {out }, 1}, i_{\text {out }, 2}, v_{\text {out }, 1}$ and $v_{\text {out }, 2}$ are also shown in Figure 5-21. It can be observed $v_{\text {out }, 1}$ and $v_{\text {out }, 2}$ have increments of $80 \mathrm{mV}$ and $50 \mathrm{mV}$ respectively, and $i_{\text {out }, 1}$ and $i_{\text {out }, 2}$ have increments of $8 \mathrm{~mA}$ and $5 \mathrm{~mA}$ correspondingly during the $85 \mathrm{~ms}$ time slot. When $\bar{i}_{i, 1}$ and $\bar{i}_{i, 2}$ reach to the steady state again, $i_{\text {out }, 1}, i_{\text {out }, 2}, v_{\text {out }, 1}$ and $v_{\text {out }, 2}$ recover back to the previous values.

\subsection{Conclusion}

In this chapter, a power sharing and cross regulation suppression method for the SIMIMO DC-DC converter has been proposed. The power sharing and cross regulation suppression are achieved based on MPC method, PI-based power sharing method and time-multiplexing method. The detailed development of the proposed method has been presented systematically in this chapter. In order to estimate the performance of the proposed method, a SI-DIDO buck converter platform has been built in Matlab/Simulink for simulation, as well as a hardware prototype for experiment. 
Simulation and experimental test cases including steady-state operation and dynamic performance have been conducted. In the steady-state operation, four conditions with different input and output voltages have been studied. The voltage ripples are suppressed less than $5 \%$ of the corresponding voltage references, and the second input current ripple is regulated less than $8.2 \%$ of the current reference. For the dynamic performance verification, it consists of line regulation, load regulation, response to output voltage reference step change and response to input current reference step change. All simulation and experimental results have demonstrated that the proposed method is able to perform the power sharing and cross regulation suppression successfully and effectively. 


\section{CHAPTER 6 DYNAMIC MODEL PREDICTIVE VOLTAGE CONTROL FOR SIDO FLYBACK CONVERTER}

In this chapter, a dynamic model predictive voltage control (DMPVC) method is adopted to regulate the SIDO flyback converter.

\subsection{State-SPACE Models AND CONSTRAINTS ANALysis}

\subsubsection{SIDO Flyback Converter}

The SIDO flyback converter is shown in Figure 6-1(a). In primary side: $V_{i n}$ denotes the DC voltage source, $i_{P}$ is the current through the primary winding, $L_{M}$ is the magnetization inductance with an ESR $R_{L}$, and $S$ is the MOSFET switch. In the secondary side: $R_{L 1}$ and $R_{L 2}$ are the ESRs of the corresponding secondary windings, $i_{S 1}$ and $i_{S 2}$ are the currents through the secondary windings, $D_{1}$ and $D_{2}$ are the freewheeling diodes, $C_{1}$ and $C_{2}$ are the filter capacitors, $R_{1}$ and $R_{2}$ are the load resistors, and $v_{1}$ and $v_{2}$ are the output voltages. The turns of the primary winding, secondary windings of branch 1 and branch 2 are $n_{P}, n_{S 1}$ and $n_{S 2}$ respectively. In conventional SIDO flyback converter, the ratio of $v_{1}$ and $v_{2}$ is determined by the turns $n_{S 1}$ and $n_{S 2}$ of the secondary windings.

In the proposed SIDO flyback converter, two MOSFET switches $S_{1}$ and $S_{2}$ are adopted for selecting the branch using time-multiplexing strategy. With the help of these two switches and the proposed DMPVC method, $v_{1}$ and $v_{2}$ are independent regulated, i.e., the relationship of $v_{1}$ and $v_{2}$ is independent to the turns ratio $n_{S 1} / n_{S 2}$, which means that the relationship of $v_{1}$ and $v_{2}$ is not coupled together anymore.

\subsubsection{State-space Model for Flyback Converter}

Figure 6-1(b), (c) and (d) show the three operational states of the proposed SIDO flyback converter, which are defined as follows:

State 1: $S$ is on, $S_{1}$ and $S_{2}$ are off. The transformer absorbs energy from the DC source. $C_{1}$ and $C_{2}$ are discharged through $R_{1}$ and $R_{2}$ because $D_{1}$ and $D_{2}$ are reverse biased.

State 2: $S$ is off, $S_{1}$ is on and $S_{2}$ is off. The transformer sends energy to $C_{1}$ and $R_{1}$ because $D_{1}$ is forward biased, and $C_{2}$ discharge through $R_{2}$ because $D_{2}$ is reverse biased. 


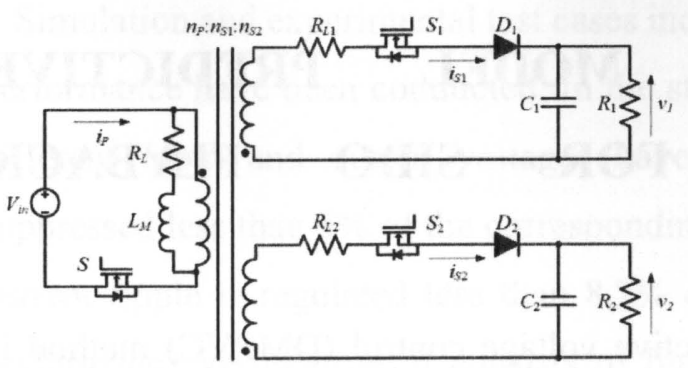

(a)

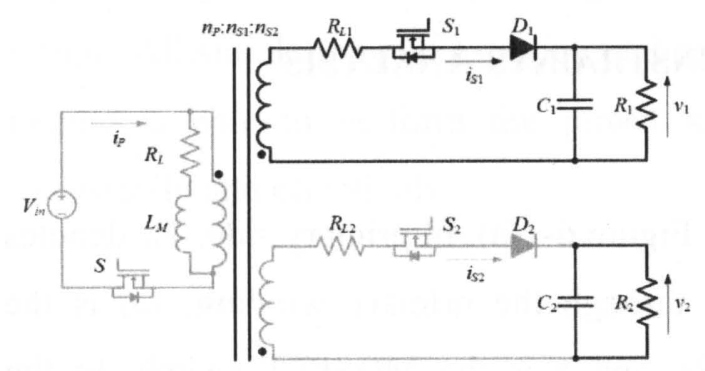

(c)

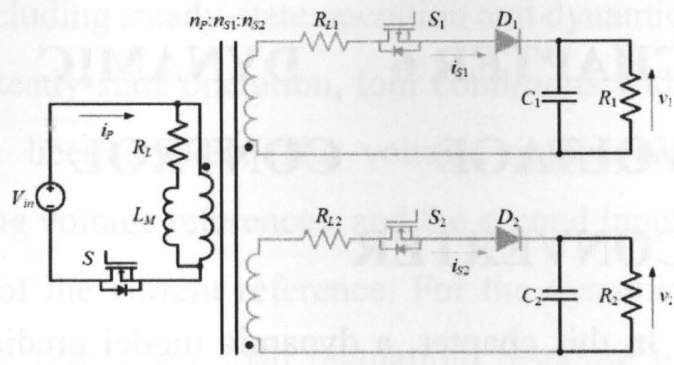

(b)

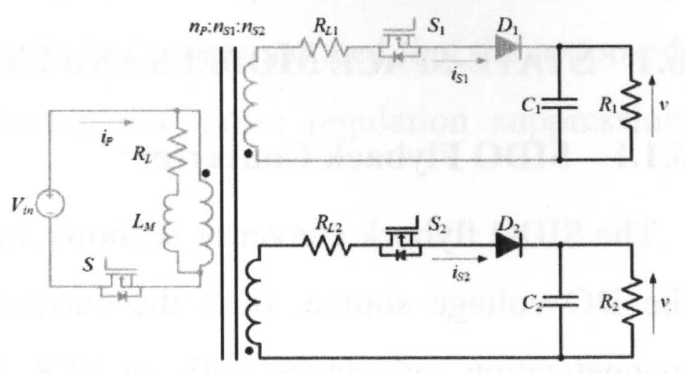

(d)

Figure 6-1: Architecture and operational states of the proposed SIDO flyback converter. (a) Architecture of SIDO flyback converter. (b) State 1. (c) State 2. (d) State 3.

State 3: $S$ is off, $S_{1}$ is off and $S_{2}$ is on. Similarly, $C_{2}$ and $R_{2}$ are supplied by the transformer, and $C_{1}$ is discharged.

The corresponding differential equations to describe the three states are listed as follows:

$$
\begin{gathered}
L_{M} \frac{d i_{p}}{d t}=S V_{i n}-i_{P} R_{L} \\
C_{i} \frac{d v_{i}}{d t}=(1-S) S_{i} I_{S, i}-\frac{v_{i}}{R_{i}}, i=1,2
\end{gathered}
$$

The waveforms of the current and control signal are shown in Figure 6-2. It can be seen that when there is a transition from state 1 to state 2 or state 3 , there will be a sudden changes of $i_{P}$ and $i_{S, i}$ so that these current waveforms are not continuous. The relationship of $i_{P}$ and $i_{S, i}$ during the aforementioned state transition follows the equation below.

$$
i_{S, i}=\frac{n_{P} i_{P} S_{i}}{n_{S, i}}
$$

Because the MPC strategy adopted in this thesis is a discrete time algorithm which has finite control sets, a control time step $T_{S}$ is set for real-time operation. It is imperative to convert the equations into discrete format. Assuming that $t$ is $k$, the statespace parameters are defined as $v_{i}(k), i_{P}(k), i_{S, i}(k), S(k)$ and $S_{i}(k)$ for the discretization. 


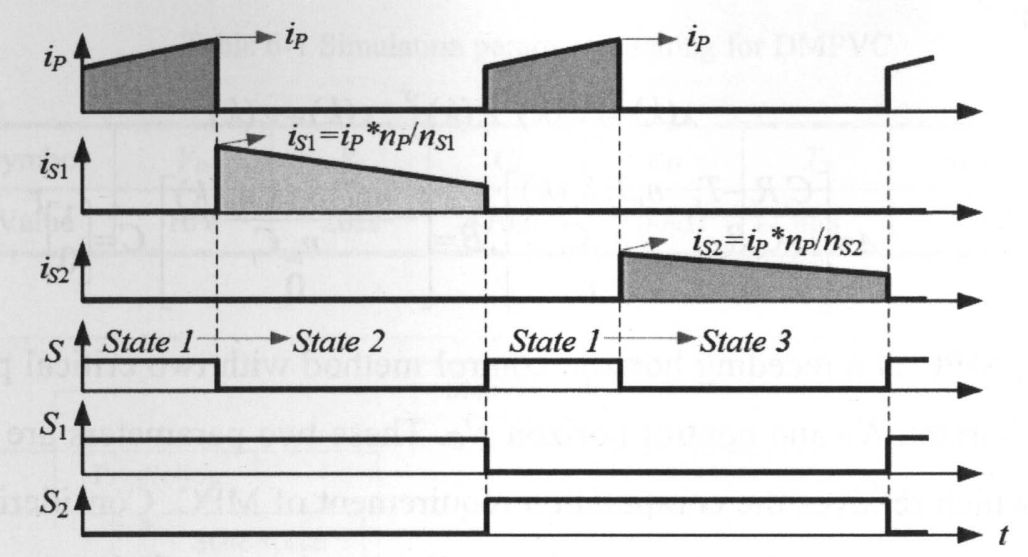

Figure 6-2: Current and control signal waveforms of the proposed flyback converter.

Moreover, considering the uncontinuous waveform of $i_{S, i}$ and (6.3), the discretization of (6.2) for prediction can be obtained as follows:

When $S(k-1)=0, i_{S, i}(k)$ can be directly used to predict the $v_{i}(k+1)$ when $S(k)$ is 1 or 0 as below.

$$
\left\{\begin{array}{l}
v_{i}(k+1)=\frac{C_{i} R_{i}-T_{S}}{C_{i} R_{i}} v_{i}(k)+\frac{T_{S} S_{i}(k)}{C_{i}} i_{S, i}(k), S(k)=0 \\
v_{i}(k+1)=\frac{C_{i} R_{i}-T_{S}}{C_{i} R_{i}} v_{i}(k), S(k)=1
\end{array}\right.
$$

When $S(k-1)=1, i_{S, i}(k)$ is 0 as shown in Figure 6-2. However, if the next control signals $S(k)=0$ and $S_{\mathrm{i}}(k)=1, i_{S, i}(k)$ for prediction follows (6.3) rather than 0 . Therefore, the prediction process when $S(k)$ is 1 or 0 is shown below.

$$
\left\{\begin{array}{l}
v_{i}(k+1)=\frac{C_{i} R_{i}-T_{S}}{C_{i} R_{i}} v_{i}(k)+\frac{n_{P} T_{S} S_{i}(k)}{n_{S, i} C_{i}} i_{P}(k), S(k)=0 \\
v_{i}(k+1)=\frac{C_{i} R_{i}-T_{S}}{C_{i} R_{i}} v_{i}(k), S(k)=1
\end{array}\right.
$$

By rewriting (6.4) and (6.5), two state-space models 1 and 2 can be obtained as follows:

when $S(k-1)=0$

$$
\begin{aligned}
& x(k+1)=A x(k)+B S(k) \\
& y(k)=C x(k)
\end{aligned}
$$

$$
\begin{gathered}
x(k)=\left[v_{i}(k) i_{S, i}(k)\right]^{T}, y(k)=v_{i}(k) \\
A=\left[\begin{array}{cc}
\frac{C_{i} R_{i}-T_{S}}{C_{i} R_{i}} & \frac{T_{S} S_{i}(k)}{C_{i}} \\
0 & 1
\end{array}\right], B=\left[\begin{array}{c}
-\frac{T_{S} S_{i}(k) i_{S, i}(k)}{C_{i}} \\
0
\end{array}\right], C=\left[\begin{array}{l}
1 \\
0
\end{array}\right]^{T}
\end{gathered}
$$


when $S(k-1)=1$

$$
\begin{gathered}
x(k)=\left[v_{i}(k) i_{P}(k)\right]^{T}, y(k)=v_{i}(k) \\
A=\left[\begin{array}{cc}
\frac{C_{i} R_{i}-T_{S}}{C_{i} R_{i}} & \frac{n_{P} T_{S} S_{i}(k)}{n_{S i} C_{i}} \\
0 & 1
\end{array}\right], B=\left[\begin{array}{c}
-\frac{n_{P} T_{S} S_{i}(k) i_{P}(k)}{n_{S i} C_{i}} \\
0
\end{array}\right], C=\left[\begin{array}{l}
1 \\
0
\end{array}\right]^{T}
\end{gathered}
$$

Moreover, MPC is a receding horizon control method with two critical parameters, i.e., predict horizon $N_{P}$ and control horizon $N_{C}$. These two parameters are set as 1 in this thesis, which relieves the computation requirement of MPC. Considering the two parameters $N_{P}=1, N_{C}=1$ and re-defining the predicted voltages $v_{i}(k+1)$ as $v_{P, i}(k+1)$, and the cost function $J$ is imperative as below.

$$
J=\sum_{i=1}^{2} S_{i}(k)\left|v_{P, i}(k+1)-v_{R e f, i}\right|
$$

The control action $S(k)$ is the optimal one if it can minimize the cost function $J$.

\subsubsection{Priority Calculation Algorithm and Constraints}

\subsubsection{Priority calculation}

As shown in model 1 (6.7) and model $2(6.8)$, the switch $S_{i}(k)$ is regarded as a constant for prediction. This is because $S_{i}(k)$ has a longer period (actually integer times) than $S(k)$ as shown in Figure 6-2 considering time-multiplexing for the dual output. Moreover, for determining the value of $S_{i}(k)$, there is a priority calculation algorithm as follows:

$$
\left\{\begin{array}{l}
S_{1}(k)=1, S_{2}(k)=0, \text { if }\left|v_{1}(k)-v_{\text {Ref. }}\right| \geq\left|v_{2}(k)-v_{\text {Ref } 2}\right| \\
S_{1}(k)=0, S_{2}(k)=1, \text { if }\left|v_{1}(k)-v_{R e f, 1}\right|<\left|v_{2}(k)-v_{\text {Ref }, 2}\right|
\end{array}\right.
$$

where $v_{R e f, 1}$ and $v_{R e f, 2}$ are the voltage references of $v_{1}$ and $v_{2}$ respectively. Although the aforementioned models help to predict the future states based on several sets of control actions $S(k)$, a cost function is indispensable to select the optimal one from all sets.

\subsubsection{Constraints}

One advantage of the MPC method is that the constraints of the control and output signals can be set according to practical situation. For the DMPVC method, the constraints of $S(k)$ are defined to obtain the proper control signals. The control signal constraints are

$$
S(k) \in\{0,1\}
$$


CHAPTER 6 DYNAMIC MODEL PREDICTIVE VOLTAGE CONTROL FOR SIDO FLYBACK CONVERTER

Table 6-1 Simulation parameters setting for DMPVC

\begin{tabular}{c|c|c|c|c|c|c}
\hline \hline Symbol & $V_{i n}$ & $R_{i}$ & $C_{i}$ & $L_{M}$ & $T_{S}$ & $n_{P}: n_{S, 1}: n_{S, 2}$ \\
\hline Value & $10 \mathrm{~V}$ & $20 \Omega$ & $470 \mu \mathrm{F}$ & $100 \mu \mathrm{H}$ & $4 \mu \mathrm{S}$ & $1: 1: 1$ \\
\hline \hline
\end{tabular}

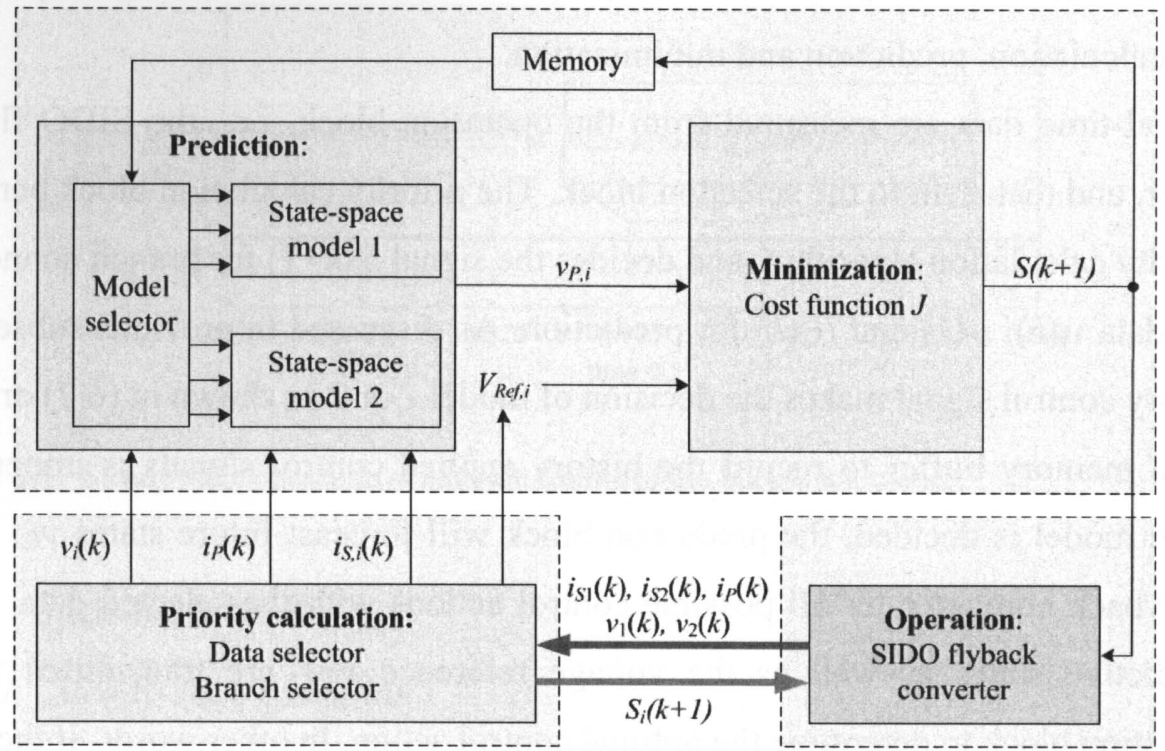

Figure 6-3: The proposed DMVPC controller for the SIDO flyback converter.

Moreover, in practical situation, the transformer has the current rating $I_{c r}$ of secondary side. If the secondary current $i_{S, i}$ exceeds $I_{c r}$, the transformer will be damaged seriously. Therefore, it is important to set an upper bound for $i_{S, i}$. Another consideration is setting the constraint for the primary current $i_{P}$ considering the relationship of $i_{P}$ and $i_{S, i}$ as shown in (6.3), which further guarantees that the secondary current $i_{S, i}$ is over the current rating $I_{c r}$. Hence, the constraint for $i_{S, i}$ is set as follows:

$$
\left\{\begin{array}{l}
i_{s, i} \leq \beta I_{c r} \\
i_{p} \leq \alpha I_{c r}
\end{array}\right.
$$

where $\alpha$ and $\beta$ are the adjustable coefficients, and they fulfills the relationship as follows:

$$
\left\{\begin{array}{c}
0<\alpha<\left(\frac{n_{P}}{n_{S, i}} \beta, 1\right)_{\min } \\
0<\beta<1
\end{array}\right.
$$

By properly setting the values of $\alpha$ and $\beta$, the secondary current $i_{S, i}$ is regulated in a suitable and proper varying range so that the transformer is protected. 


\subsection{DMPVC METHOD FOR SIDO FLYBACK CONVERTER}

Due to the two state-space models, which are dynamically used depending on realtime data, therefore, the proposed method is defined as a dynamic MPC method. With the state-space models, priority calculation algorithm and cost function, the proposed DMPVC method is developed. As shown in Figure 6-3, there are four blocks: operation, priority calculation, prediction and minimization.

The real-time data are measured from the operation block, i.e., the SIDO flyback converter, and then sent to the selection block. The priority calculation block performs the priority calculation algorithm, and decides the signal $S_{i}(k+1)$ for branch connection and the data $v_{i}(k), i_{P}(k)$ and $i_{S, i}(k)$ for prediction. As discussed in previous subsection, the history control signal makes the decision of model 1 or 2 as shown in (6.7) or (6.8). Hence, a memory buffer to record the history applied control signals is imperative. Once the model is decided, the prediction block will forecast future states $v_{P, i}$ of the SIDO flyback converter for all possible control actions with the selected data. Then, the predictive states as well as the voltage reference $v_{R e f, i}$ are transmitted to the minimization block to determine the optimal control action. In other words, if the $v_{P, i}$ is able to minimize the cost function $J$ in (6.10), the corresponding control action is the best choice to regulate the converter tracking the voltage reference. Therefore, the DMPVC method is able to respond to the real-time data dynamically and fast so that it can regulate two output branches of the SIDO flyback converter independently.

\subsection{Simulation Results}

In order to verify the performance of the DMPVC method for the SIDO flyback converter, simulation is conducted based on MATLAB/Simulink and the results are presented in this section. Simulation parameters of the SIDO flyback converter for the DMPVC method are listed in Table 6-1. As the aforementioned discussion, the SIDO flyback converter using the DMPVC method has three operation modes, i.e., buck-buck, buck-boost and boost-boost. Consequently, simulation cases of the steady-state operation and load regulation under these three modes are conducted and discussed in this section. Moreover, the mode transformations from buck-buck to buck-boost and from buck-boost to boost-boost are also performed to further estimate the performance of the proposed DMPVC method. 

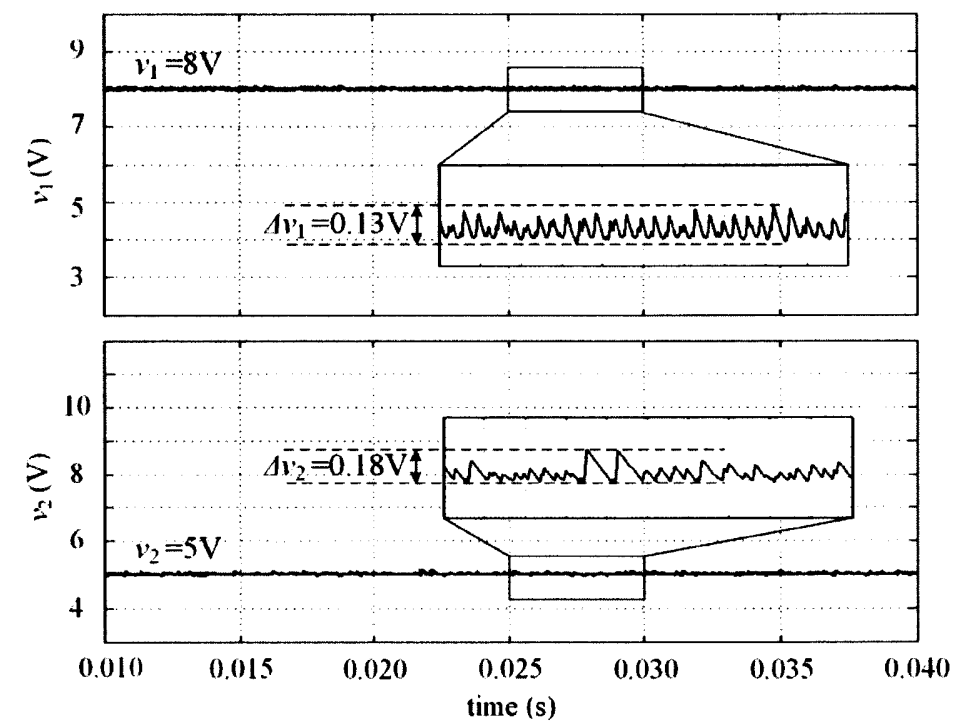

Figure 6-4: Steady-state operation simulation results of the buck-buck mode.

\subsubsection{Buck-buck Mode}

\subsubsection{Steady-state operation}

The simulation results of the buck-buck mode based on the SIDO flyback converter are shown in Figure 6-4. The initial voltage references $v_{R e f, 1}$ and $v_{R e f, 2}$ are set as $8.0 \mathrm{~V}$ and $5.0 \mathrm{~V}$ respectively for the buck-buck mode operation. It can be observed that the output voltages are $v_{1}=8 \mathrm{~V}$ and $v_{2}=5 \mathrm{~V}$, which track the preset voltage references closely.

Moreover, the corresponding voltage ripples are $\Delta v_{1}=0.13 \mathrm{~V}$ and $\Delta v_{2}=0.18 \mathrm{~V}$, which are $1.62 \%$ of $v_{R e f, 1}$ and $3.6 \%$ of $v_{R e f, 2}$. Hence, the DMPVC method regulates the SIDO flyback converter to work in buck-buck mode successfully.

\subsubsection{Load Regulation}

The load regulation simulation results of the buck-buck mode are shown in Figure 65. It can be seen that the voltage references $v_{R e f, 1}$ and $v_{R e f, 2}$ are also set as $8 \mathrm{~V}$ and $5 \mathrm{~V}$ respectively for the buck-buck mode operation.

When $t=0.05 \mathrm{~s}$, there are step changes in $R_{1}$ and $R_{2}$ from $20 \Omega$ to $13.3 \Omega$, i.e., $50 \%$ increment of two loads respectively. Correspondingly, the output current $i_{1}$ is increased from $0.4 \mathrm{~A}$ to $0.6 \mathrm{~A}$, and the output current $i_{2}$ is increased from $0.25 \mathrm{~A}$ to $0.375 \mathrm{~A}$. In addition, the voltage ripples are $\Delta v_{1}=0.12 \mathrm{~V}$ and $\Delta v_{2}=0.13 \mathrm{~V}$ before the step changes in loads, and the voltage ripples are increased after the step changes, which are $\Delta v_{1}=0.25 \mathrm{~V}$ and $\Delta v_{2}=0.26 \mathrm{~V}$, i.e., $3.1 \%$ of $v_{R e f, 1}$ and $5.2 \%$ of $v_{R e f, 2}$. Therefore, the proposed DMPVC 

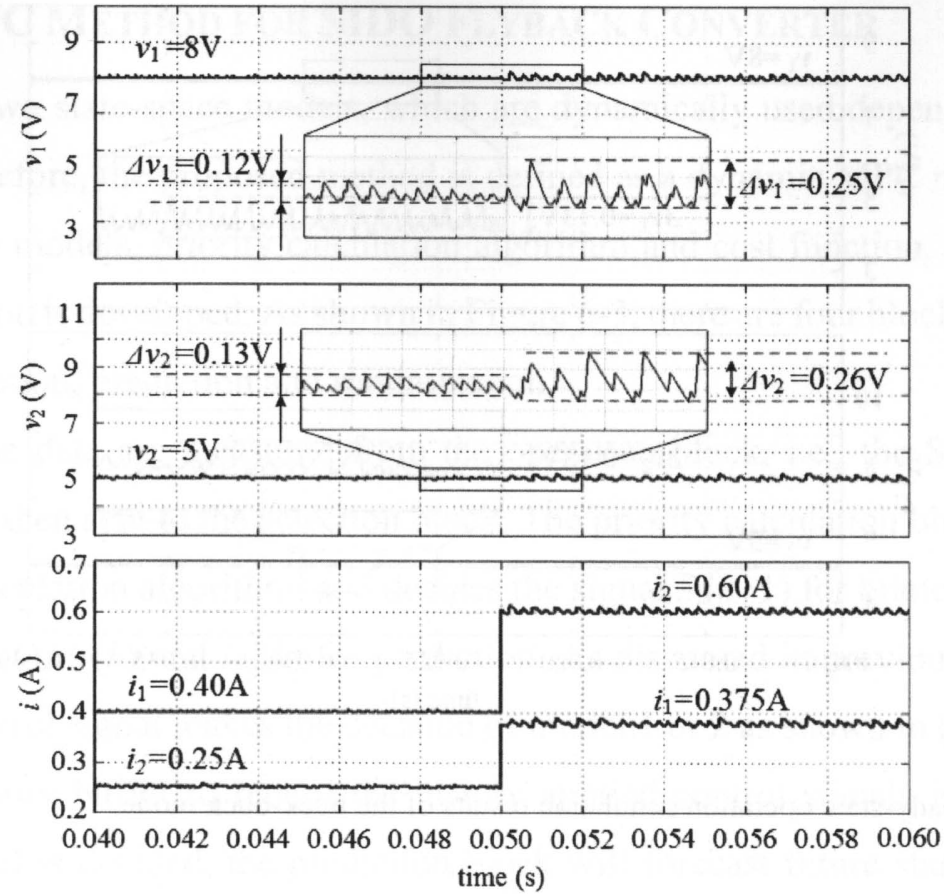

Figure 6-5: Load regulation simulation results of the buck-buck mode.

method is able to perform the load regulation successfully when there is a sudden increment of $100 \%$ in load in the buck-buck mode.

\subsubsection{Buck-boost Mode}

\subsubsection{Steady-state operation}

The simulation results of the buck-boost mode are shown in Figure 6-6. It can be seen that the voltage references $v_{\text {Ref,1 }}$ and $v_{R e f, 2}$ are set as $12 \mathrm{~V}$ and $7 \mathrm{~V}$ respectively for the buck-buck mode operation. Consequently, the output voltages are $v_{1}=12 \mathrm{~V}$ and $v_{2}=7 \mathrm{~V}$, which track the preset voltage references closely.

Moreover, the corresponding voltage ripples are $\Delta v_{1}=0.22 \mathrm{~V}$ and $\Delta v_{2}=0.25 \mathrm{~V}$, which are $1.83 \%$ of $v_{R e f, 1}$ and $3.12 \%$ of $v_{R e f, 2}$. Hence, the DMPVC method has the capability to regulate the SIDO flyback converter to work in the buck-boost mode successfully.

\subsubsection{Load Regulation}

The load regulation simulation results of the buck-boost mode are shown in Figure 6-7. At the beginning, the voltage references $v_{R e f, 1}$ and $v_{R e f, 2}$ are also set as $12.0 \mathrm{~V}$ and 7.0V respectively for the buck-boost mode operation, and the corresponding current are $i_{1}=0.60 \mathrm{~A}$ and $i_{2}=0.35 \mathrm{~A}$. Moreover, the initial voltage ripples are $\Delta v_{1}=0.19 \mathrm{~V}$ and 

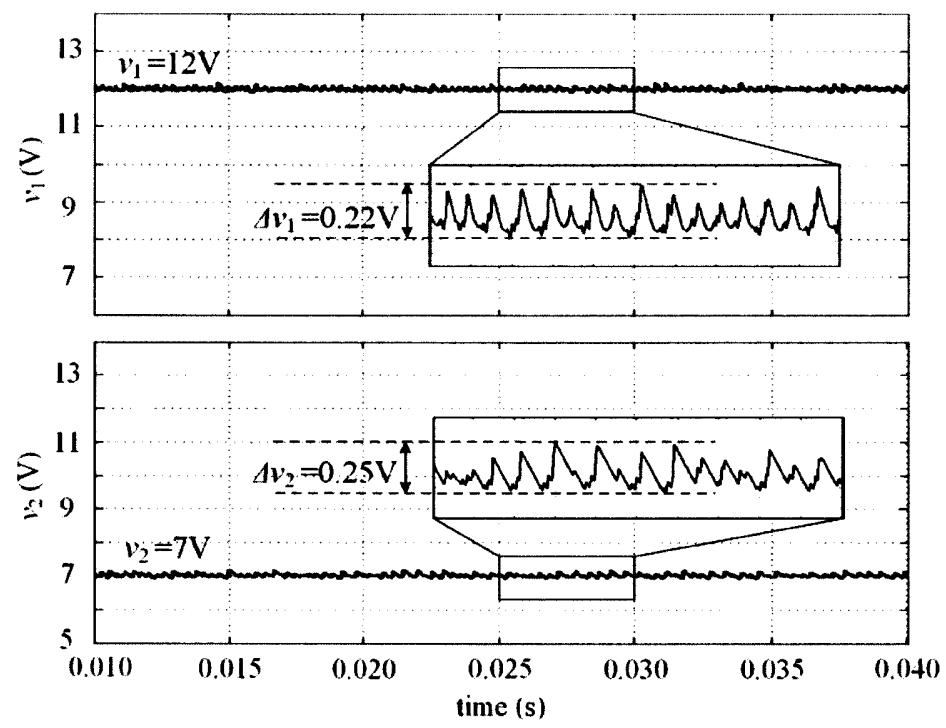

Figure 6-6: Steady-state operation simulation results of the buck-boost mode.
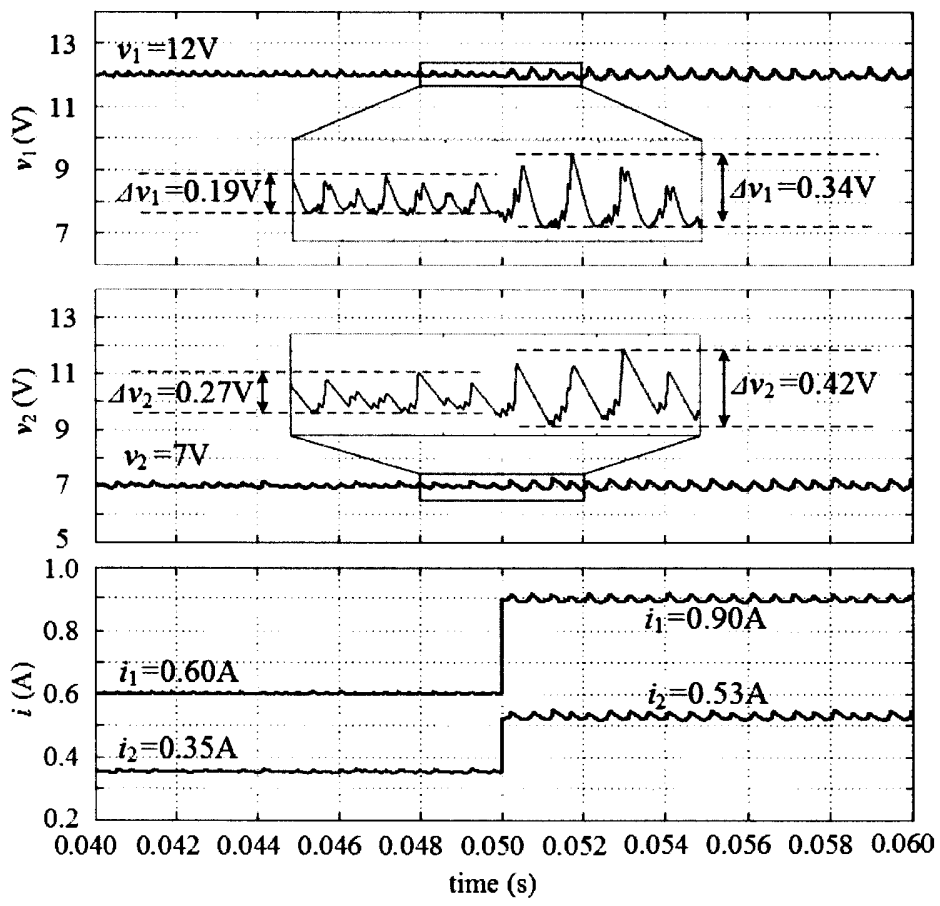

Figure 6-7: Load regulation simulation results of the buck-boost mode.

$\Delta v_{2}=0.27 \mathrm{~V}$, therefore, the SIDO flyback converter works in buck-boost mode well. There are step changes in $R_{1}$ and $R_{2}$ from $20 \Omega$ to $13.3 \Omega$, i.e., $50 \%$ increment of two loads respectively. Hence, the current $i_{1}$ is increased from $0.60 \mathrm{~A}$ to $0.90 \mathrm{~A}$ and the current $i_{2}$ is increased from $0.35 \mathrm{~A}$ to $0.53 \mathrm{~A}$ when $t=0.05 \mathrm{~s}$. Accordingly, the voltage ripples $\Delta v_{1}$ and $\Delta v_{2}$ are increased to from $0.19 \mathrm{~V}$ to $0.34 \mathrm{~V}$ and from $0.27 \mathrm{~V}$ to $0.42 \mathrm{~V}$, i.e., $2.83 \%$ of $v_{R e f, 1}$ and $6 \%$ of $v_{R e f, 2}$. Therefore, the DMPVC method regulates the output 

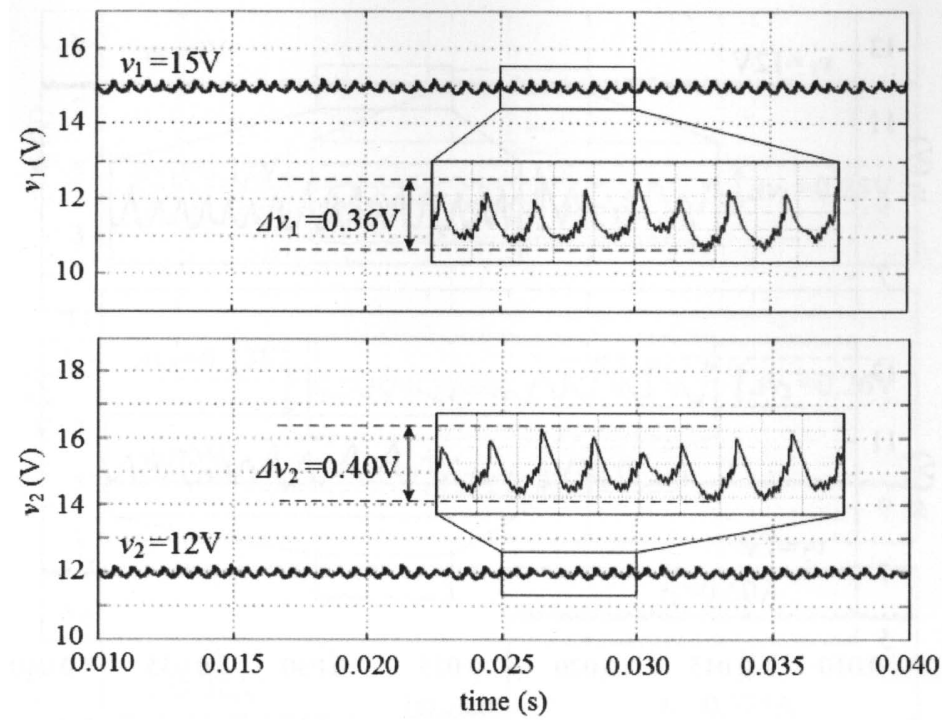

Figure 6-8: Steady-state operation simulation results of the boost-boost mode.

voltages tracking the references well before and after the step change in load, which means that the load regulation is completed successfully in the buck-boost mode.

\subsubsection{Boost-boost Mode}

\subsubsection{Steady-state operation}

The simulation results of the boost-boost mode are shown in Figure 6-8. It can be seen that the voltage references $v_{R e f, 1}$ and $v_{R e f, 2}$ are set as $15 \mathrm{~V}$ and $12 \mathrm{~V}$ respectively for the boost-boost mode operation. It can be observed the output voltages are $v_{1}=15 \mathrm{~V}$ and $v_{2}=12 \mathrm{~V}$, which track the preset voltage references closely.

Moreover, the corresponding voltage ripples are $\Delta v_{1}=0.36 \mathrm{~V}$ and $\Delta v_{2}=0.40 \mathrm{~V}$, which are $2.4 \%$ of $v_{\text {Ref. } 1}$ and $3.3 \%$ of $v_{\text {Ref,2. }}$. Hence, the DMPVC method regulates the SIDO flyback converter to work in the boost-boost mode successfully.

\subsubsection{Load Regulation}

In the this simulation case, the voltage references $v_{R e f, 1}$ and $v_{R e f, 2}$ are set as $15.0 \mathrm{~V}$ and $12.0 \mathrm{~V}$ for $v_{1}$ and $v_{2}$ so that the SIDO flyback converter works in boost-boost mode, and the load regulation simulation results are shown in Figure 6-9. The initial voltage ripples are $\Delta v_{1}=0.35 \mathrm{~V}$ and $\Delta v_{2}=0.32 \mathrm{~V}$, and the output currents are $i_{1}=0.75 \mathrm{~A}$ and $i_{2}=0.60 \mathrm{~A}$. Similarly, step changes from $20 \Omega$ to $13.3 \Omega$ in $R_{1}$ and $R_{2}$, i.e., $50 \%$ increment of loads, happen when $\mathrm{t}=0.05 \mathrm{~s}$, and the currents $i_{1}$ and $i_{2}$ are increased to $1.12 \mathrm{~A}$ and $0.90 \mathrm{~A}$ respectively. Due to the increment of loads, the voltage ripples $\Delta v_{1}$ and $\Delta v_{2}$ are increased to $0.62 \mathrm{~V}$ and $0.58 \mathrm{~V}$ respectively, which are $4.13 \%$ of $v_{R e f, 1}$ and $4.83 \%$ of $v_{R e f, 2 .}$. 


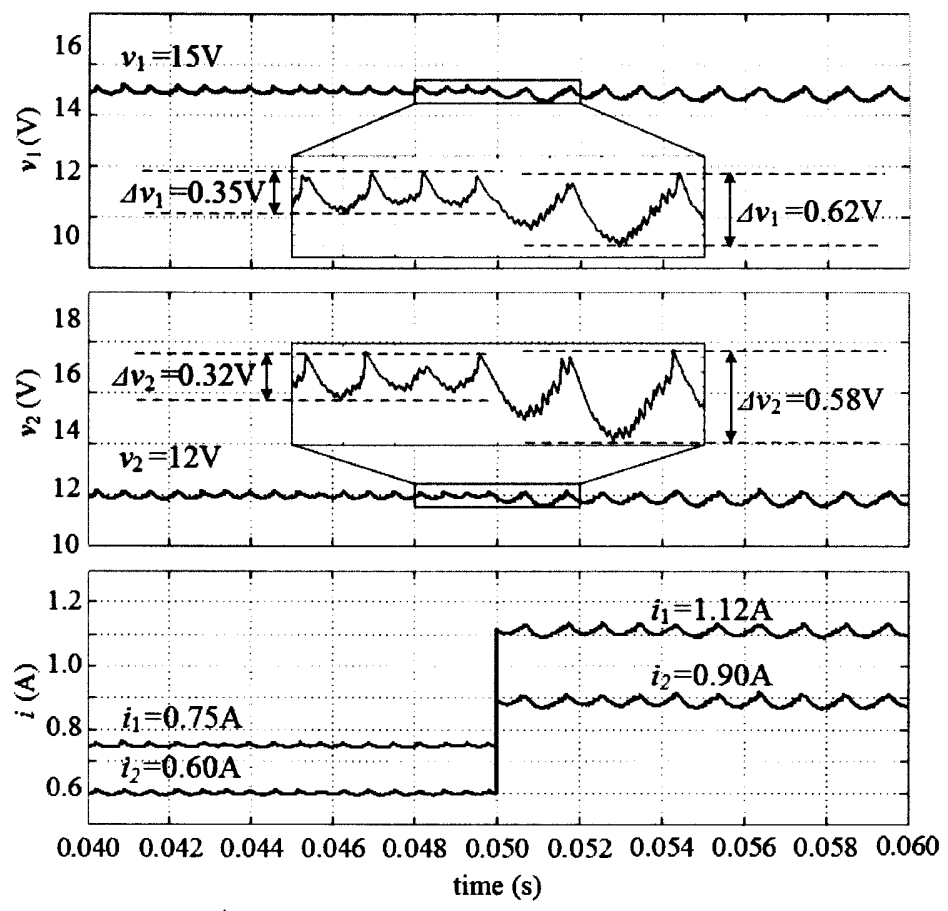

Figure 6-9: Load regulation simulation results of the boost-boost mode.

The load regulation of the SIDO flyback converter in the boost-boost mode is operated well by using the proposed DMPVC method.

\subsubsection{Mode Transition}

\subsubsection{Buck-buck Mode to Buck-boost Mode}

The mode transformation from the buck-buck mode to the buck-boost mode is simulated, and the results are shown in Figure 6-10. The initial voltage references $v_{R e f, 1}$ and $v_{R e f, 2}$ are set as $8 \mathrm{~V}$ and $5 \mathrm{~V}$ respectively so that the SIDO flyback converter works in buck-buck mode. When $t=0.05 \mathrm{~s}$, the voltage reference $v_{\text {Ref, } 1}$ is increased from $8 \mathrm{~V}$ to $12 \mathrm{~V}$, therefore, the SIDO flyback converter enters the buck-boost mode. It can be observed from Figure 6-10 that it takes about 1.2ms of the proposed DMPVC method to complete the mode transition. Moreover, the output $v_{2}$ has a large ripple of $0.75 \mathrm{~V}$ due to the mode transition. After the completion of the transition, $v_{2}$ is regulated back to $5 \mathrm{~V}$ by the proposed DMPVC method. In summary, the proposed DMPVC method has the capability to perform the mode transition from the buck-buck mode to the buckboost mode of the SIDO flyback converter. 

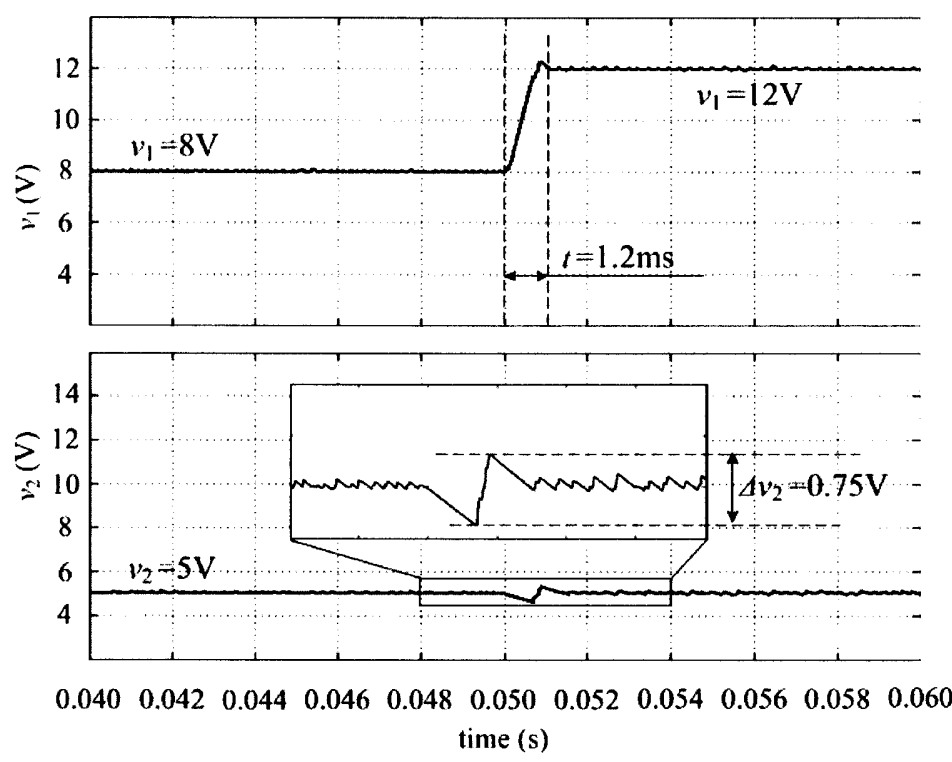

Figure 6-10: Mode transformation from buck-buck mode to buck-boost mode.
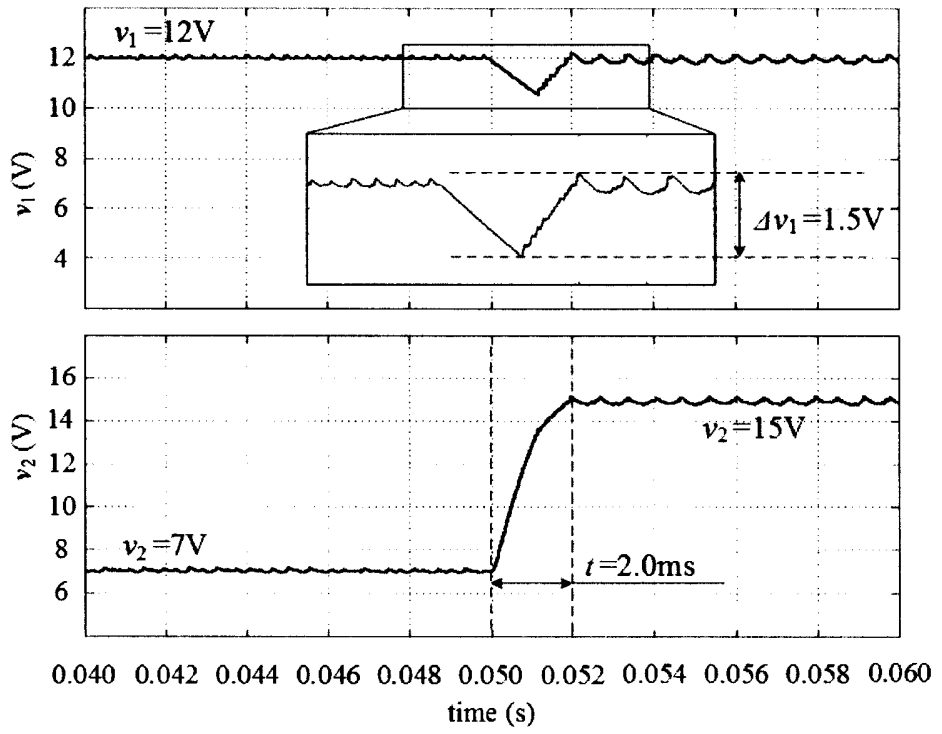

Figure 6-11: Mode transformation from buck-boost mode to boost-boost mode.

\subsubsection{Buck-boost Mode to Boost-boost Mode}

The mode transition from the buck-boost mode to the boost-boost mode is simulated, and the results are shown in Figure 6-11. The initial voltage references $v_{R e f, 1}$ and $v_{R e f, 2}$ are set as $12 \mathrm{~V}$ and $7 \mathrm{~V}$ respectively so that the SIDO flyback converter works in the buck-boost mode. When $t=0.05 \mathrm{~s}$, the voltage reference $v_{R e f, 2}$ is increased from $7 \mathrm{~V}$ to $15 \mathrm{~V}$, which means that the SIDO flyback requires the buck-boost mode operation. It can be observed from Figure 6-11 that it takes about $2.0 \mathrm{~ms}$ of the proposed DMPVC 
method to complete the mode transition. Moreover, the output $v_{1}$ has a large voltage drop of $1.5 \mathrm{~V}$ due to the mode transition. However, the proposed DMPVC method regulates the output voltage $v_{1}$ back to $12 \mathrm{~V}$ after the completion of the transition. Therefore, the mode transition from the buck-boost mode to the boost-boost mode is successfully performed by the proposed DMPVC method.

\subsection{Conclusion}

In this chapter, a DMPVC method for the SIDO flyback converter has been proposed. In order to implement the proposed method, the state-space models of the SIDO flyback converter have been developed, as well as the cost function, priority calculation algorithm and constraint. Based on the state-space models, the simulation platform of the SIDO flyback converter and the controller has been built in the Matlab/Simulink. Consequently, several simulation cases have been conducted to verify the performance of the proposed DMPVC method.

Three basic simulation cases include buck-buck mode, buck-boost mode and boostboost mode. In each mode, the steady-state and load regulation have been conducted with the simulation platform for a comprehensive estimation on the performance of the proposed DMPVC method. In the simulation results, the output voltage ripples have been significantly suppressed compared to the reference voltages in all study cases. Moreover, the load regulation has been verified in the three modes, which shows that the proposed method copes with the step change in load successfully. Finally, the mode transition simulation cases, including from the buck-buck mode to the buck-boost mode and the buck-boost mode to the boost-boost mode, have been conducted. The simulation results have shown that the proposed DMPVC method can handle the step change in voltage reference well to complete the mode transition. In summary, all the simulation results have demonstrated that the proposed DMPVC method is an effective method, which has robust, dynamic and rapid response capability, to regulate the SIDO flyback converter. 


\section{CHAPTER 7 CONCLUSIONS AND FUTURE WORKS}

\subsection{Conclusions}

Nowadays, multi-port DC-DC converters have been adopted in HRESs. The multiports DC-DC converters have the ability to interface multiple power sources and multiple loads using less components and smaller circuit size, compared to the SISO DC-DC converter. Especially, the multi-port DC-DC converters with single inductor/ transformer further minimize the size of HRESs since the multiple loads sharing the same inductor/transformer. However, the multiple loads will influence each other through the residual energy on the shared inductor/transformer from every load, which is so-called cross regulation problem.

The MPC method has the features of on-line optimization, varying switching frequency and easy incorporation of constraints. These features facilitate the dynamic and fast response of the MPC method, which makes it possible to solve the cross regulation problem. Consequently, several control methods deriving from the MPC have been presented for different kinds of multi-port DC-DC converters.

In this thesis, based on the MPC theory, the MPVC method has been developed for the SIMO DC-DC converter. Since the SIMO DC-DC converter drives multiple loads using a single shared inductor, the cross regulation is inevitable. The proposed MPVC is targeted to solve the cross regulation problem. The state-space model with the corresponding cost function and constraints of the MPVC method has been developed for a SIMO DC-DC converter. Consequently, the MPVC controller for a universal SIMO DC-DC converter has been developed, and it can generate the optimal control signals so that the cross regulation is suppressed significantly. Moreover, since the control signal of the MPVC method is aperiodic, the design of inductor and capacitors in the SIMO DC-DC converter is different from the conventional control methods with fixed switching frequency. Hence, the values of inductor and capacitors are analyzed for the MPVC method.

Simulation platform in Matlab/Simulink has been built to study the proposed MPVC method. In the simulation, the influences of the MPC parameters (including predict horizon, control horizon and Lagrange multiplier) on the voltage ripple, the steady-state operation and the dynamic performance of the proposed MPVC method have been studied to guide the hardware implementation. The proposed MPVC method has also 
been implemented in a hardware prototype of the SIDO buck converter, and several experimental test cases have been presented to estimate the performance of the proposed MPVC method including steady-state operation and dynamic performance. The simulation and experimental results have demonstrated that the proposed MPVC method can regulate the SIMO DC-DC converter successfully with reduced cross regulation and fast response to the step change in load and reference.

Similarly, the MPCC method has been proposed for the SIMO DC-DC converter. With the proposed MPCC method, the converter is able to drive multiple loads with different output currents following the preset current references. Based on this MPCC method, a battery charge equalization method has been further developed for seriesconnected battery cells in EVs. In order to provide proper references for each battery cell, the corresponding current reference assignment algorithms for the MPCC method in $\mathrm{CC}$ charging stage and CV charging stage have also been proposed considering the real-time SOCs information. With the MPCC method and current reference assignment algorithms, the charging equalization method is able to realize the individual charging operation for each battery cell using the SIMO DC-DC converter, so that the imbalance problem of the series-connected battery cells in EVs can be solved.

The performance of the proposed charge equalization has been verified by several simulation studies based on a SIMO buck converter in Matlab/Simulink. The simulation results have demonstrated that the $\mathrm{CC}$ charging and $\mathrm{CV}$ charging with the tolerable ripples can be achieved by the proposed MPCC method. Moreover, the ability of the proposed MPCC method to solve the cross regulation problem of the SIMO DC-DC converter with battery cells is also estimated. The simulation results have shown that the output currents track the assigned current references closely, i.e., the cross regulation is significantly suppressed when driving multiple battery cells with different demand. This capability contributes to the ability of individual charging operation for charging equalization. In order to conduct an overall verification of the proposed charge equalization method, two different situations, i.e., small SOC difference and large SOC difference, are set for simulation. The simulation results have demonstrated that the proposed charge equalization method is able to balance the series-connected battery cells under different SOC situations.

The MPC method has been further extended from SIMO DC-DC convertor to SIMIMO DC-DC converter. Due to the multiple inputs of SI-MIMO DC-DC converter, the regulation of input power sharing ratios should be considered. In this thesis, a power 
sharing and cross regulation suppression method for the SI-MIMO DC-DC converter has been presented. The functions of power sharing and cross regulation suppression are achieved by combining the MPC method, PI-based power sharing method and timemultiplexing method. The detailed development of the proposed method has been presented systematically.

A SI-DIDO buck converter platform has been built in Matlab/Simulink for simulation to estimate the performance of the proposed method. With the guidance of the simulation results, a hardware prototype has also been developed to implement the proposed method for experimental validation. With the simulation and experimental platforms, steady-state operation and dynamic performance have been conducted. In the steady-state operation, four conditions with different input and output voltages have been studied. All simulation and experimental results have demonstrated that the power sharing ratios are successfully regulated and the cross regulation suppression is significantly reduced by the proposed method. For the dynamic performance verification, it consists of line regulation, load regulation, response to output voltage reference step change and response to input current reference step change. The proposed method is capable to handle the variations in input voltages, loads, power sharing ratios and output voltage references of the SI-MIMO DC-DC converter.

The flyback converter replaces the inductor with a transformer in conventional DCDC converters. This transformer isolates the input power source and the output load. Moreover, the voltage ratio is enlarged by the turns ratio of the transformer. Therefore, the adoption of flyback converter with multiple ports in HERSs is promising. In this thesis, a DMPVC method for the SIDO flyback converter has been proposed. The DMPVC method is different from the previous proposed MPVC and MPCC methods, because it needs to switch models during the real-time operation. This model switch results from the different current values of the primary side and secondary side with different turns of windings. Moreover, the turns ratio of two secondary windings in the transformer limits the two output voltages of the SIDO flyback converter. The proposed DMPVC method is able to regulate the output voltages individually, so that the SIDO flyback converter can work in buck-buck mode, buck-boost mode and boost-boost mode.

Analogously, the state-space models of the SIDO flyback converter, as well as the cost function, priority calculation algorithm and constraints have been presented in this thesis. The SIDO flyback converter has been built in the Matlab/Simulink for the 


\subsubsection{Parameter Selection Using Particle Swarm Optimization}

As discussed previously, the quadratic programming algorithm is adopted to solve the optimization problem of the cost function, which has high computational complexity. Recently, the particle swarm optimization (PSO) algorithm has also been proposed to solve the optimization problem of MPC [138], Compared to the quadratic programming algorithm, the PSO algorithm is a stochastic population-based technique. Moreover, it has some advantages over quadratic programming algorithm, such as parallel capabilities, global search, simple implementation, less space complexity, etc. Therefore, PSO is a promising method to solve the optimization problem in MPC-based method in this thesis. 


\section{REFERENCES}

[1] B. K. Bose, "Global warming: energy, environmental pollution, and the impact of power electronics," IEEE Industrial Electronics Magazine, vol. 4, no. 1, pp. 6-17, Mar. 2010.

[2] G. T. Heydt, kR. Ayyanar, K. W. Hedman, and V. Vittal, "Electric power and energy engineering: the first century," Proceedings of the IEEE, vol. 100, no. special centennial issue, pp. 1315-1328, May 2012.

[3] A. Izadian, N. Girrens, and P. Khayyer, "Renewable energy policies: a brief review of the latest U.S. and E.U. policies," IEEE Industrial Electronics Magazine, vol. 7, no. 3, pp. 2134, Sept. 2013.

[4] N. L. Panwar, S. C. Kaushik, and S. Kothari, "Role of renewable energy sources in environmental protection: a review," Renewable and Sustainable Energy Reviews, vol. 15, no. 3, pp. 1513-1524. Apr. 2011.

[5] International energy statistics EIA's portal for detailed country and regional energy data [Online]. Available: http://tonto.eia.doe.gov/ cfapps/ipdbproject/IEDIndex3.cfm.

[6] M. J. Barradale, "Impact of public policy uncertainty on renewable energy investment: Wind power and the production tax credit," Energy Policy, vol. 38, no. 12, pp. 7698-7709, Dec. 2010.

[7] A. E. Jones, M. Irwin, and A. Izadian, "Incentives for microgeneration development in the U.S. and Europe," in Proc. IEEE Industrial Electronics Conference, 2010, pp. 3018-3021.

[8] K. Malmedal, B. Kroposki, and P. K. Sen, "Energy policy act of 2005 and its impact on renewable energy applications in USA," in Proc. IEEE Power Engineering Society General Meeting, 2007, pp. 1-8.

[9] C. Klessmann, A. Held, M. Rathmann, and M. Ragwitz, "Status and perspectives of renewable energy policy and deployment in the European Union-what is needed to reach the 2020 targets?" Energy Policy, vol. 39, no. 12, pp. 7637-7657, Dec. 2011.

[10] J. C. Wu and C. W. Chou, "A solar power generation system with a seven-level inverter," IEEE Trans. Power Electronics, vol. 29, no. 7, pp. 3454-3462, Jul. 2014.

[11] G. J. Shirek and B. A. Lassiter, "Photovoltaic power generation: modeling solar plants' load levels and their effects on the distribution system," IEEE Industry Applications Magazine, vol. 19, no. 4, pp. 63-72, Aug. 2013.

[12] J. C. Wu, K. D. Wu, H. L. Jou, and S. K. Chang, "Small-capacity grid-connected solar power generation system," IET Power Electronics, vol. 7, no. 11, pp. 2717-2725, Nov. 2014. 
13] J. C. Wu and Y. H. Wang, "Power conversion interface for small-capacity wind power generation system," IET Generation, Transmission and Distribution, vol. 8, no. 4, pp. 689696, Apr. 2014.

14] J. Ribrant and L. M. Bertling, "Survey of failures in wind power systems with focus on Swedish wind power plants during 1997-2005," IEEE Trans. Energy Conversion, vol. 22, no. 1, pp.167-173, Jun. 2007.

15] Y. Duan and R. G. Harley, "Present and future trends in wind turbine generator designs," in Proc. Power Electronics and Machines in Wind Applications, 2009, pp. 1-6.

16] X. Sun, M. Cheng, Y. Zhu, and L. Xu, "Application of electrical variable transmission in wind power generation system," IEEE Trans. Industry Applications, vol. 49, no. 3, pp. 1299-1307, Jun. 2013.

17] T. K. A. Brekken, H. T. Ozkan-Haller, and A. Simmons, "A methodology for large-scale ocean wave power time-series generation," IEEE Journal of Oceanic Engineering, vol. 37, no. 2, pp. 294-300, Apr. 2012.

18] A. J. Garrido, I. Garrido, M. Amundarain, M. Alberdi, and M. Sen, "Sliding-mode control of wave power generation plants," IEEE Trans. Industry Applications, vol. 48, no. 6, pp. 2372-2381, Dec. 2012.

19] M. H. Nehrir, C. Wang, K. Strunz, H. Aki, R. Ramakumar, J. Bing, Z. Miao, and Z. Salameh, "A review of hybrid renewable/alternative energy systems for electric power generation: configurations, control, and applications," IEEE Trans. Sustainable Energy, vol. 2, no. 4, pp. 392-403, Oct. 2011.

20] Hawaii Natural Energy Institute, University of Hawaii at Manoa, Hawaii hydrogen power park [Online]. Available: http://www.hnei.hawaii.edu/hhpp.asp.

21] T. Nakken, L. R. Strand, E. Frantzen, R. Rohden, and P. O. Eide, "The Utsira windhydrogen system-Operational experience," in Proc. European Wind Energy Conference, 2006 ,

22] Ø. Ulleberg, T. Nakken, and A. Eté, "The wind/hydrogen demonstration system at Utsira in Norway: evaluation of system performance using operational data and updated hydrogen energy system modeling tools," International Journal of Hydrogen Energy, vol. 35, no. 5, pp. 1841-1852, Mar. 2010.

23] G. Tina, S. Gagliano, and S. Raiti, "Hybrid solar/wind power system probabilistic modeling for long-term performance assessment," Solar Energy, vol. 80, no. 5, pp. 578-588, May 2006. 
[24] D. J. Lee and L. Wang, "Small-signal stability analysis of an autonomous hybrid renewable energy power generation/energy storage system part I: time-domain simulations," IEEE Trans. Energy Conversion, vol. 23, no. 1, pp. 311-320, Mar. 2008.

[25] D. K. Khatod, V. Pant, and J. Sharma, "Analytical approach for well-being assessment of small autonomous power systems with solar and wind energy sources," IEEE Trans. Energy Conversion, vol. 25, no. 2, pp. 535-545, Jun. 2010.

[26] A. Arabali, M. Ghofrani, M. E. Amoli, and M. S. Fadali, "Stochastic performance assessment and sizing for a hybrid power system of solar/wind/energy storage," IEEE Trans. Sustainable Energy, vol. 5, no. 2, pp. 363-371, Apr. 2014.

[27] L. Xu, X. Ruan, C. Mao, B. Zhang, and Y. Luo, "An improved optimal sizing method for wind-solar-battery hybrid power system," IEEE Trans. Sustainable Energy, vol. 4, no. 3, pp. 774-785, Jul. 2013.

[28] J. Wang and F. Yang, "Optimal capacity allocation of standalone wind/solar/battery hybrid power system based on improved particle swarm optimization algorithm," IET Renewable Power Generation, vol. 7, no. 5, pp. 443-448, Sep. 2013.

[29] T. Hirose and H. Matsuo, "Standalone hybrid wind-solar power generation system applying dump power control without dump load," IEEE Trans. Industrial Electronics, vol. 59, no. 2, pp. 988-997, Feb. 2012.

[30] M. L. Rahman, S. Oka, and Y. Shirai, "Hybrid power generation system using offshorewind turbine and tidal turbine for power fluctuation compensation (HOT-PC)," IEEE Trans. Sustainable Energy, vol. 1, no. 2, pp. 92-98, Jul. 2010.

[31] A. M. O. Haruni, M. Negnevitsky, M. E. Haque, and A. Gargoom, "A novel operation and cntrol strategy for a standalone hybrid renewable power system," IEEE Trans. Sustainable Energy, vol. 4, no. 2, pp. 402-413, Apr. 2013.

[32] H. Matsuo, W. Lin, F. Kurokawa, T. Shigemizu, and N. Watanabe, "Characteristics of the multiple-input DC-DC converter," IEEE Trans. Industrial Electronics, vol. 51, no. 3, pp. 625-631, Jun. 2004.

[33] H. Behjati and A. Davoudi, "A MIMO topology with series outputs: an interface between diversified energy sources and diode-clamped multilevel inverter," in Proc. IEEE Applied Power Electronics Conference and Exposition, 2012, pp. 5-9.

[34] L. Situ, "Electric vehicle development: the past, present \& future," in Proc. IEEE International Conference on Power Electronics Systems and Applications, 2009, pp. 20-22. 
35] Q. Zhang, "The trend of international oil price and China's coping strategies," in Proc. IEEE International Conference on Electronics, Communications and Control, 2011, pp. 9 11.

36] K. Rajashekara, "Present status and future trends in electric vehicle propulsion technologies," IEEE Journal of Emerging and Selected Topics in Power Electronics, vol. 1, no. 1, pp. 310, Mar. 2013.

37] A. Khaligh and Z. H. Li, "Battery, ultracapacitor, fuel cell, and hybrid energy storage systems for electric, hybrid electric, fuel cell, and plug-in hybrid electric vehicles: state of the art," IEEE Trans. Vehicular Technology, vol. 59, no. 6, pp. 2806-2814, Jul. 2010.

38] J. Du and M. Ouyang, "Review of electric vehicle technologies progress and development prospect in China," Electric Vehicle Symposium and Exhibition, 2013, pp. 17-20.

39] T. Zhang, W. Chen, Z. Han, and Z. G. Cao, "Charging scheduling of electric vehicles with local renewable energy under uncertain electric vehicle arrival and grid power price," IEEE Trans. Vehicular Technology, vol. 63, no. 6, pp. 2600-2612, Jul. 2014.

40] C. Jin, X. Sheng, and P. Ghosh, "Optimized electric vehicle charging with intermittent renewable energy sources," IEEE Journal of Selected Topics in Signal Processing, vol. 8, no. 6, pp. 1063-1072, Dec. 2014.

41] F. Mwasilu, J. J. Justo, E. K. Kim, T. D. Do, and J. W. Jung, "Electric vehicles and smart grid interaction: a review on vehicle to grid and renewable energy sources integration," Renewable and Sustainable Energy Reviews, vol. 34, pp. 501-516, Jun. 2014.

42] A. Y. Saber and G. K. Venayagamoorthy, "Plug-in vehicles and renewable energy sources for cost and emission reductions," IEEE Trans. Industrial Electronics, vol. 58, no. 4, pp. 1229-1238, Apr. 2011.

43] J. Cao and A. Emadi, "A new battery/ultracapacitor hybrid energy-storage system for electric, hybrid, and plug-in hybrid electric vehicles," IEEE Trans. Power Electronics, vol. 27, no. 1, pp.122-132, Jan. 2012.

44] R. V. Haaren, "Assessment of electric cars' range requirements and usage patterns based on driving behavior recorded in the national household travel survey of 2009," Fu Foundation School of Engineering and Applied Science, 2011.

45] C. S. Si and X. L. Xu, "Research of the charge mode of electric automobile charging station and the key technology of the control system," in Proc. IEEE International Conference on Consumer Electronics, Communications and Networks, 2012, pp. 2788-2790. 
[46] P. T. Krein and R. S. Balog, "Life extension through charge equalization of lead-acid batteries," in Proc. IEEE Telecommunications Energy Conference, 2002, pp. 516-523.

[47] H. S. Park, C. H. Kim, K. B. Park, G. W. Moon, and J. H. Lee, "Design of a charge equalizer based on battery modularization," IEEE Trans. Vehicular Technology, vol. 58, no. 7, pp. 3216-3223, Sep. 2009.

[48] L. Y. Wang, L. F. Wang, C. L. Liao, and J. Liu, "Research on battery balance system applied on HEV," in Proc. IEEE Vehicle Power and Propulsion Conference, 2009, pp. 1788-1791.

[49] J. L. Sun, R. G. Lu, G. Wei, B. L. Xu, and C. B. Zhu, "A high efficiency equalizer based on forward converter for series connected battery string," in Proc. Vehicle Power and Propulsion Conference, 2012, pp. 376- 379.

[50] C. H. Kim, M. Y. Kim, and G. W. Moon, "A modularized charge equalizer using a battery monitoring IC for series-connected Li-Ion battery strings in electric vehicles," IEEE Trans. Power Electronics, vol. 28, no. 8, pp. 3779-3787, Aug. 2013.

[51] U. Iraola, I. Aizpuru, L. Gorrotxategi, J. M. C. Segade, A. E. Larrazabal, and I. Gil, "Influence of voltage balancing on the temperature distribution of a Li-Ion battery module," IEEE Trans. Energy Conversion, vol. 30, no. 2, pp. 507-514, Jun. 2015.

[52] R. J. Wai, C. Y. Lin, J. J. Liaw, and Y. R. Chang, "Newly designed ZVS multi-input converter," IEEE Trans. Industrial Electronics, vol. 58, no. 2, pp. 555-566, Feb. 2011.

[53] R. J. Wai, C. Y. Lin, and B. H. Chen, "High-efficiency DC-DC converter with two input power sources," IEEE Trans. Power Electronics, vol. 27, no. 4, pp. 1862-1875, Apr. 2012.

[54] A. Khaligh, J. Cao, and Y. J. Lee, "A multiple-input DC-DC converter topology," IEEE Trans. Power Electronics, vol. 24, no. 3, pp. 862-868, Mar. 2009.

[55] C. Shi, B. Miller, K. Mayaram, and T. Fiez, "A multiple-input boost converter for lowpower energy harvesting," IEEE Trans. Circuits and Systems II: Express Briefs, vol. 58, no. 12, pp. 827-831, Dec. 2011.

[56] Q. Wang, J. Zhang, X. Ruan, and K. Jin, "Isolated single primary winding multiple-input converters," IEEE Trans. Power Electronics, vol. 26, no. 12, pp. 3435-3442, Dec. 2011.

[57] R. Ahmadi and M. Ferdowsi, "Double-input converters based on H-bridge cells: derivation, small-signal modeling, and power sharing analysis," IEEE Trans. Circuits and Systems I: Regular Papers, vol. 59, no. 4, pp. 875-888, Apr. 2012.

[58] F. Nejabatkhah, S. Danyali, S. H. Hosseini, M. Sabahi, and S. M. Niapour, "Modeling and control of a new three-input DC-DC boost converter for hybrid PV/FC/Battery power system," IEEE Trans Power Electronics, vol. 27, no. 5, pp. 2309-2324, May 2012. 
[59] C. N. Onwuchekwa and A. Kwasinski, "A modified-time-sharing switching technique for multiple-input DC-DC converters," IEEE Trans. Power Electronics, vol. 27, no. 11, pp. 4492-4502, Nov. 2012.

[60] R. Ahmadi, H. Zargarzadeh, and M. Ferdowsi, "Nonlinear power sharing controller for a double-input H-bridge-based buckboost-buckboost converter," IEEE Trans. Power Electronics, vol. 28, no. 5, pp. 2402-2414, May 2013.

[61] L. Kumar and S. Jain, "Multiple-input DC/DC converter topology for hybrid energy system," IET Power Electronics, vol. 6, no. 8, pp. 1483-1501, Sep. 2013.

[62] R. J. Wai and B. H. Chen, "High-efficiency dual-input interleaved DC-DC converter for reversible power sources," IEEE Trans. Power Electronics, vol. 29, no. 6, pp. 2903-2921, Jun. 2014.

[63] T. Anno and H. Koizumi, "Double-input bidirectional DC/DC converter using cell-voltage equalizer with flyback transformer," IEEE Trans. Power Electronics, vol. 30, no. 6, pp. 2923-2934, Jun. 2015.

[64] L. Xian and Y. Y. Wang, "Exact steady-state analysis in multiple-input converters applied with diverse time-sharing switching schemes," IET Power Electronics, vol. 8, no. 5, pp. 724-734, May 2015.

[65] D. Ma, W. H. Ki, C. Y. Tsui, and P. K. T. Mok, "Single-inductor multiple-output switching converters with time-multiplexing control in discontinuous conduction mode," IEEE $J$. Solid-State Circuits, vol. 38, no. 1, pp. 89-100, Jan. 2003.

[66] D. Ma, W. H. Ki, and C. Y. Tsui, "A pseudo-CCM/DCM SIMO switching converter with freewheel switching," IEEE J. Solid-State Circuits, vol. 38, no. 6, pp. 1007-1014, Jun. 2003.

[67] Y. Zhang and D. Ma, "Integrated SIMO DC-DC converter with on-line charge meter for adaptive PCCM operation," in Proc. IEEE International Symposium on Circuits and Systems (ISCAS), 2011, pp. 245-248.

[68] Y. Zhang and D. Ma, "Adaptive pseudo-continuous conduction mode operation schemes and circuit designs for single-inductor multiple-output switching converters," Analog Integrated Circuits and Signal Processing, vol. 72, no. 2, pp. 419-432, Aug. 2012.

[69] H. P. Le, C. S. Chae, K. C. Lee, S. W. Wang, and G. H. Cho, "A single-inductor switching dc-dc converter with five output and ordered power-distributive control," IEEE J. SolidState Circuits, vol. 42, no.12, pp. 2706-2714, Dec. 2007. 
[70] A. Pizzutelli and M. Ghioni, "Novel control technique for single inductor multiple output converters operating in CCM with reduced cross-regulation," in Proc. IEEE Appl. Power Electron. Conf. Expo., 2008, pp.1502-1507.

[71] D. Trevisan, P. Mattavelli, and P. Tenti, "Digital control of single-inductor multiple-output step-down dc-dc converters in CCM," IEEE Trans. Industrial Electronics, vol. 55, no. 9, pp. 3476-3483, Sep. 2008.

[72] W. W. Xu, Y. Li, X. Gong, Z. L. Hong, and D. Killat, "A dual-mode single-inductor dualoutput switching converter with small ripple," IEEE Trans. Power Electronics, vol. 25, no. 3, pp. 614-623, Mar. 2010.

[73] Z. Shen, X. Chang. W. Wang, X. Tan, N. Yan, and H. Min, "Predictive digital current control of single-inductor multiple-output converters in CCM with low cross regulation," IEEE Trans. Power Electronics, vol. 27, no. 4, pp. 1917-1925, Apr. 2012.

[74] P. Patra, J. Ghosh, and A. Patra, "Control scheme for reduced cross regulation in singleinductor multiple-output dc-dc converters," IEEE Trans. Industrial Electronics, vol. 60, no. 11, pp. 5095-5104, Nov. 2013.

[75] J. D. Dasika, B. Bahrani, M. Saeedifard, A. Karimi, and A. Rufer, "Multivariable control of single-inductor dual-output buck converters," IEEE Trans. Power Electronics, vol. 29, no. 4, pp. 2061-2070, Apr. 2014.

[76] Y. Zhang and D. Ma, "A fast-response hybrid SIMO power converter with adaptive current compensation and minimized cross-regulation," IEEE J. Solid-State Circuits, vol. 49, no. 5, pp. 1242-1255, May 2014.

[77] X. Liu, J. Xu, Z. Chen, and N. Wang, "Single-inductor dual-output buck-boost power factor correction converter," IEEE Trans. Industrial Electronics, vol. 62, no. 2, pp. 943-952, Feb. 2015.

[78] T. Bhattacharya, V. S. Giri, K. Mathew, and L. Umanand, "Multiphase bidirectional flyback converter topology for hybrid electric vehicles," IEEE Trans. Industrial Electronics, vol. 56, no. 1, pp. 78-84, Jan. 2009.

[79] D. C. Lu and V. G. Agelidis, "Photovoltaic-battery-powered DC bus system for common portable electronic devices," IEEE Trans. Power Electronics, vol. 24, no. 3, pp. 849-855, Mar. 2009.

[80] Y. Hu, W. Xiao, W. Cao, B. Ji, and D. J. Morrow, "Three-port DC-DC converter for standalone photovoltaic systems," IEEE Trans. Power Electronics, vol. 30, no. 6, pp. 3068-3076, Jun. 2015. 
81] S. C. Moon, G. B. Koo, and G. W. Moon, "A new control method of interleaved singlestage flyback AC-DC converter for outdoor LED lighting systems," IEEE Trans. Power Electronics, vol. 28, no. 8, pp. 4051-4062, Aug. 2013.

82] S. Buso, G. Spiazzi, and F. Sichirollo, "Study of the asymmetrical half-bridge flyback converter as an effective line-fed solid-state lamp driver," IEEE Trans. Industrial Electronics, vol. 61, no. 12, pp. 6730-6738, Dec. 2014.

83] J. P. Hong and G. W. Moon, "A digitally controlled soft valley change technique for a flyback converter," IEEE Trans. Industrial Electronics, vol. 62, no. 2, pp. 966-971, Feb. 2015.

84] D. Maksimovic, R. W. Erickson, and C. Griesbach, "Modelling of cross-regulation in converters containing coupled inductor," IEEE Trans. Power Electronics, vol. 15, no. 4, pp. 607-615, Jul. 2000.

85] C. Ji, Jr. M. Smith, K. M. Smedley, and K. King, "Cross regulation in flyback converters: analytic model and solution," IEEE Trans. Power Electronics, vol. 16, no. 2, pp. 231-239, Mar. 2001.

86] J. J. Lee and B. H. Kwon, "DC-DC converter using a multiple-coupled inductor for low output voltages," IEEE Trans. Industrial Electronics, vol. 54, no. 1, pp. 467-478, Feb. 2007.

87] L. Hang, S. Wang, Y. Gu, W. Yao, and Z. Lu, "High cross-regulation multioutput LLC series resonant converter with magamp postregulator," IEEE Trans. Industrial Electronics, vol. 58, no. 9, pp. 3905-3913, Sep. 2011.

88] J. K. Kim, S. W. Choi, C. E. Kim, and G. W. Moon, "A new standby structure using multioutput full-bridge converter integrating flyback converter," IEEE Trans. Industrial Electronics, vol. 58, no. 10, pp. 4763-4767, Oct. 2011.

89] H. S. Kim, J. H. Jung, J. W. Baek, and H. J. Kim, "Analysis and design of a multioutput converter using asymmetrical PWM half-bridge flyback converter employing a parallelseries transformer," IEEE Trans. Industrial Electronics, vol. 60, no. 8, pp. 3115-3125, Aug. 2013.

90] J. K. Kim, J. B. Lee, and G. W. Moon, "Zero-voltage switching multioutput flyback converter with integrated auxiliary buck converter," IEEE Trans. Power Electronics, vol. 29, no. 6, pp. 300-310, Jun. 2014.

91] F. F. Edwin, W. Xiao, and V. Khadkikar, "Dynamic modeling and control of interleaved flyback module-integrated converter for PV power applications," IEEE Trans. Industrial Electronics, vol. 61, no. 3, pp. 1377-1388, Mar. 2014. 
[92] H. Y. Li and H. C. Chen, "Dynamic modeling and controller design for a single-stage singleswitch parallel boost-flyback-flyback converter," IEEE Trans. Power Electronics, vol. 27, no. 2, pp. 816-827, Feb. 2012.

[93] H. Behjati and A. Davoudi, "A multi-port dc-dc converter with independent outputs for vehicular applications," in Proc. IEEE Vehicle Power and Propulsion Conference, 2011, pp. $1-5$.

[94] D. Gunasekaran and L. Umanand, "Integrated magnetics based multi-port bidirectional DCDC converter topology for discontinuous-mode operation," IET Power Electronics, vol. 5, no. 7, pp. 935-944, Aug. 2012.

[95] H. Behjati and A. Davoudi, "Single-stage multi-port DC-DC converter topology," IET Power Electronics, vol. 6, no. 2, pp. 392-403, Feb. 2013.

[96] H. Behjati and A. Davoudi, "Power sharing between diversified energy sources and loads using a multiple-input multiple-output DC-DC converter," IEEE Trans. Industry Applications, vol. 49, no. 6, pp. 2761-2772, Dec. 2013.

[97] H. Behjati and A. Davoudi, "A multiple-input multiple-output DC-DC converter," IEEE Trans. Industry Applications, vol. 49, no. 3, pp. 1464-1479, Jun. 2013.

[98] S. Danyali, S. H. Hosseini, and G. B. Gharehpetian, "New extendable single-stage multiinput DC-DC/AC boost converter," IEEE Trans. Power Electronics, vol. 29, no. 2, pp. 775788, Feb. 2014.

[99] H. Shao, X. Li, C. Y. Tsui, and W. H. Ki, "A novel single-inductor dual-input dual-output DC-DC converter with PWM control for solar energy harvesting system," IEEE Trans. Very Large Scale Integration Systems, vol. 22, no. 8, pp. 1693-1704, Aug. 2014.

[100] J. Cao, N. Schofield, and A. Emadi, "Battery balancing methods: A comprehensive review," in Proc. IEEE Vehicle Power and Propulsion Conference, 2008, pp. 1-6.

[101] Y. S. Lee and M. W. Cheng, "Intelligent control battery equalization for series connected lithium-ion battery strings," IEEE Trans. Industrial Electronics, vol. 52, no. 5, pp. 1297 1307, Oct. 2005.

[102] Y. S. Lee and G. T. Cheng, "Quasi-resonant zero-current-switching bidirectional converter for battery equalization applications," IEEE Trans. Power Electronics, vol. 21, no. 5, pp. 1213-1224, Sep. 2006.

[103] W. L. Chen and S. R. Cheng, "Optimal charge equalization control for series-connected batteries," IET Generation, Transmission and Distribution, vol. 7, no. 8, pp. 843-854, Aug. 2013. 
104] W. Hong, K. S. Ng, J. H. Hu, and C. S. Moo, "Charge equalization of battery power modules in series," in Proc. IEEE International Power Electronics Conference, 2010, pp. 1568-1572.

105] C. S. Moo, K. S. Ng, and Y. C. Hsieh, "Parallel operation of battery power modules," IEEE Trans. Energy Conversion, vol. 23, no. 2, pp. 701-707, Jun. 2008.

106] H. Park, C. Kim, G. Moon, and J. Lee, "A modularized charge equalizer for an HEV lithiumion battery string," IEEE Trans. Industrial Electronics, vol. 56, no. 5, pp. 1464-1476, May 2009.

[07] C. H. Kim, M. Y. Kim, H. S. Park, and G. W. Moon, "A modularized two-stage charge equalizer with cell selection switches for series-connected lithium-ion battery string in an HEV," IEEE Trans. Power Electronics, vol. 27, no. 8, pp. 3764-3774, Aug. 2012.

[08] M. Kim, J. Kim, C. Kim, S. Cho, and G. Moon, "Automatic charge equalization circuit based on regulated voltage source for series connected lithium-ion batteries," in Proc. IEEE International Conference on Power Electronics and ECCE Asia, 2011, pp. 2248-2255.

109] Y. Hsieh, T. Liang, S. Chen, W. Horng, and Y. Chung, "A novel high-efficiency compactsize low-cost balancing method for series-connected battery applications," IEEE Trans. Power Electronics, vol. 28, no. 12, pp. 5927-5939, Dec. 2013.

110] J. Yun, T. Yeo, and J. Park, "High efficiency active cell balancing circuit with soft-switching technique for series-connected battery string," in Proc. IEEE Applied Power Electronics Conference and Exposition, 2013, pp. 3301-3304.

111] C. Hua, Y. Fang, and P. Li, "Charge equalization for series-connected LiFePO4 battery strings," IET Power Electronics, vol. 8, no. 6, pp. 1017-1025, Jun. 2015.

112] P. Cortes, M. P. Kazmierkowski, R. M. Kennel, D. E. Quevedo, and J. Rodriguez, "Predictive control in power electronics and drives," IEEE Trans. Industrial Electronics, vol. 55, no. 12, pp. 4312-4324, Dec. 2008.

113] S. Kouro, P. Cortes, R. Vargas, U. Ammann, and J. Rodriguez, "Model predictive controla simple and powerfui method to control power converters," IEEE Trans. Industrial Electronics, vol. 56, no. 6, pp.1826-1838, Jun. 2009.

114] D. Plaza, R. De Keyser, and J. Bonilla, "Model predictive and sliding mode control of a boost converter," in Proc. International Symposium on Power Electronics, Electrical Drives, Automation and Motion (SPEEDAM), 2008, pp. 37-42.

115] F. M. Oettmeier, J. Neely, S. Pekarek, R. DeCarlo, and K. Uthaichana, "MPC of switching in a boost converter using a hybrid state model with a sliding mode observer," IEEE Trans. Industrial Electronics, vol. 56, no. 9, pp. 3453-3466, Sep. 2009. 
[116] P. Karamanakos, T. Geyer, and S. Manias, "Direct voltage control of DC-DC boost converters using enumeration-based model predictive control," IEEE Trans. Power Electronics, vol. 29, no. 2, pp. 968-978, Feb. 2014.

[117] L. Tarisciotti, P. Zanchetta, A. Watson, S. Bifaretti, and J. C. Clare, "Modulated model predictive control for a seven-level cascaded H-Bridge back-to-back converter," IEEE Trans. Industrial Electronics, vol. 61, no. 10, pp. 5375-5383, Oct. 2014.

[118] D. K. Choi and K. B. Lee, "Dynamic performance improvement of AC/DC converter using model predictive direct power control with finite control set," IEEE Trans. Industrial Electronics, vol. 62, no. 2, pp. 757-767, Feb. 2015.

[119] P. Cortes, G. Ortiz, J. I. Yuz, J. Rodriguez, S. Vazquez, and L. G. Franquelo, "Model predictive control of an inverter with output LC filter for UPS applications," IEEE Trans. Industrial Electronics, vol. 56, no. 6, pp. 1875-1883, Jun. 2009.

[120] P. Cortes, A. Wilson, S. Kouro, J. Rodriguez, and H. Abu-Rub, "Model predictive control of multilevel cascaded H-bridge inverters," IEEE Trans. Industrial Electronics, vol. 57, no. 8, pp. 2691-2699, Aug. 2010.

[121] C. S. Lim, N. A. Rahim, W. P. Hew, and E. Levi, "Model predictive control of a two-motor drive with five-leg-inverter supply," IEEE Trans. Industrial Electronics, vol. 60, no. 1, pp. 54-65, Jan. 2013.

[122] V. Yaramasu, B. Wu, and J. Chen, "Model-predictive control of grid-tied four-level diodeclamped inverters for high-power wind energy conversion systems," IEEE Trans. Power Electronics, vol. 29, no. 6, pp. 2861-2873, Jun. 2014.

[123] D. E. Quevedo, R. P. Aguilera, M. A. Perez, P. Cortes, and R. Lizana, "Model predictive control of an AFE rectifier with dynamic references," IEEE Trans. Power Electron., vol. 27, no. 7, pp. 3128-3136, Jul. 2012.

[124] Y. Zhang, W. Xie, Z. Li, and Y. Zhang, "Model predictive direct power control of a PWM rectifier with duty cycle optimization," IEEE Trans. Power Electronics, vol. 28, no. 11, pp. 5343-5351, Nov. 2013.

[125] S. Kwak and J. C. Park, "Model-predictive direct power control with vector preselection technique for highly efficient active rectifiers," IEEE Trans. Industrial Informatics, vol. 11, no. 1, pp. 44-52, Feb. 2015.

[126] Y. Zhang and C. Qu, "Model predictive direct power control of PWM rectifiers under unbalanced network conditions," IEEE Trans. Industrial Electronics, vol. 62, no. 7, pp. 4011-4022, Jul. 2015. 
[127] P. Karamanakos, K. Pavlou, and S. Manias, "An enumeration-based model predictive control strategy for the cascaded H-bridge multilevel rectifier," IEEE Trans. Industrial Electronics, vol. 61, no. 7, pp. 3480-3489, Jul. 2014.

[128] D. Q. Mayne, J. B. Rawlings, C. V. Rao, and P. O. M. Scokaert, "Survey constrained model predictive control: stability and optimality," Automatica, vol. 36, pp. 789-814, Jun. 2000.

[129] J. M. Maciejowski, Predictive Control with Constraints. New Jersey, USA: Prentice Hall, 2002.

[130] D. Axehill, L. Vandenberghe, and A. Hansson, "Convex relaxations for mixed integer predictive control," Automatica, vol. 46, no. 9, pp. 1540-1545, 2010.

[131] A. Bemporad, M. Morari, V. Dua, and E. N. Pistikopoulos, "The explicit linear quadratic regulator for constrained systems," Automatica, vol. 38, no. 1, pp. 3-20, Jan. 2002.

[132] T. A. Johansen and A. Grancharova, "Approximate explicit constrained linear model predictive control via orthogonal search tree," IEEE Trans. Automatic Control, vol. 58, no. 5, pp. 810-815, May 2003.

[133] A. Bemporad and C. Filippi, "Suboptimal explicit receding horizon control via approximate multiparametric quadratic programming," Journal of Optimization Theory and Applications, vol. 117, no. 1, pp. 9-38, Apr. 2003.

[134] D. Donald and J. Castorerna. (2006, June). Buck-converter design demystified [Online]. Available: http:/l powerelectronics.com/sitefiles/powerelectronics.com/files/archive/powerelectronics.com/mag/606PET25.pdf.

[135] F. Vasca and L. Iannelli, Dynamics and Control of Switched Electronic Systems. SpringerVerlag, 2012.

[136] E. Robles, S. Ceballos, J. Pou, J. L. Martín, J. Zaragoza, and P. Ibañez, "Variable-frequency grid-sequence detector based on a quasi-ideal low-pass filter stage and a phase-locked loop," IEEE Trans. Power Electronics, vol. 25, no. 10, pp. 2552-2563, Oct. 2010.

[137] S. Golestan, M. Ramezani, J. M. Guerrero, F. D. Freijedo, and M. Monfared, "Moving average filter based phase-locked loops: performance analysis and design guidelines," IEEE Trans. Power Electronics, vol. 29, no. 6, pp. 2750-2763, Jun. 2014.

[138] F. Xu, H. Chen, X. Gong, and Q. Mei, "Fast nonlinear model predictive control on FPGA using particle swarm optimization," in IEEE Trans. on Industrial Electronics, vol. 63, no. 1, pp. 310-321, Jan. 2016. 


\section{LIST OF PUBLICATIONS}

\section{Journal publications}

[1] B. Wang, K. V. Ravi Kishore, L. Xian, X. Peng, K. T. Tan, and P. L. So, "Model predictive voltage control for single-inductor multiple-output DC-DC converter with reduced cross regulation," IEEE Trans. Industrial Electronics, vol. 63, no. 7, pp. 4187-4197, Jul. 2016.

[2] B. Wang, L. Xian, K. V. Ravi Kishore, K. J. Tseng, A. Ukil, and H. B. Gooi, "A digital method of power sharing and cross regulation suppression for singleinductor multiple-input multiple-output DC-DC converter," IEEE Trans. Industrial Electronics, Nov. 2016.

[3] K. V. Ravi Kishore, B. Wang, P. L. So and Z. Wang, "Analysis, design and implementation of an APWM ZVZCS full-bridge DC-DC converter for battery charging in electric vehicles," IEEE Trans. Power Electronics, Sep. 2016.

\section{Conference publications}

[1] B. Wang, K. T. Tan, and P. L. So, "Low cross regulation SIMO DC/DC converter with model predictive voltage control", in Proc. IEEE Power and Energy Society General Meeting (PESGM), Jul. 2013. (Best Paper Session)

[2] B. Wang, K. V. Ravi Kishore, and P. L. So, "Model predictive voltage control method for flyback converter", in Proc. IEEE India International Conference (INDICON), Dec. 2015.

[3] B. Wang, L. Xian, and K. J. Tseng, "Dynamic model predictive voltage control for single-input dual-output flyback converter", in Proc. IEEE Conference on Industrial Electronics and Applications (ICIEA), Jun. 2016.

[4] K. V. Ravi Kishore, B. Wang, and P. L. So, "A new ZVS full-bridge DC-DC converter for battery charging with reduced losses over full-load range", in Proc. IEEE India International Conference (INDICON), Dec. 2015. (Best Pape Award)

[5] Y. Wang, B. Wang, and P. L. So, "A voltage regulation method using distributec energy storage systems in LV distribution”, in Proc. IEEE International Energy Conference (ENERGYCON), Apr. 2016. 